\title{
SOVIET PRECISION TIMEKEEPING RESEARCH AND TECHNOLOGY
}

\author{
R. F. C. Vessot \\ D. W. Allan \\ S. J. B. Crampton \\ L. S. Cutler \\ R. H. Kern \\ A. O. McCoubrey \\ J. D. White
}

\section{August 1991}

SAlE Science Applications International Corporation 


\section{FOREIGN APPLIED SCIENCES ASSESSMENT CENTER}

PURPOSE

REPORTS

ORGANIZATION
The Foreign Applied Sciences Assessment Center (FASAC) is operated for the Federal Government by Science Applications International Corporation (SAIC) to improve US knowledge of foreign applied science and to increase awareness of new foreign technologies with military, economic, or political importance. Such knowledge can reduce technological surprise, can support estimates of the consequences of technology transfer, and can provide a background for US research and development decisions.

The Center directs leading US scientists in the preparation of technical assessment reports and provides continuity as a national forum for periodic reviews of foreign science research activities.

Although FASAC examines world applied science, emphasis is placed on research in the Soviet Union. The Center reports on what the Soviets call exploratory research (akin to Department of Defense 6.1 and 6.2 research), which seeks to translate developments in fundamental research into new technology. Further, as the globalization of advanced technology proceeds, more FASAC assessments are of foreign (non-Soviet) research efforts. The Center generally does not report on technology already being incorporated in engineering applications.

In addition to an assessment of the quality and emphasis of foreign research, a Center report provides milestones for monitoring subsequent progress. It also provides elements of a net technical assessment of the balance with US science, without being an out-and-out comparison.

The permanent Center staff includes the Center's Director, two Senior Scientists, a Senior Editor, a Technical Information Specialist, and an Assistant Editor. FASAC panels consist of expert consultants from academia, industry, and government, typically six to eight members per panel.

Each panel assesses the status and potential impacts of foreign applied science in a selected area. Panel members are selected by the following criteria: leading authority in the field; recent "hands-on" experience; knowledge of foreign research; and knowledge of the direction of US research programs. The panels review broad areas of applied science and then focus on particular activities of interest to their assessment. At intervals, panels are convened to reassess past FASAC topics involving areas of rapidly advancing science and technology of particular importance.

The Director and Senior Scientists help select the topics to be assessed, select the Panel Chairmen, and guide and assist in the preparation of panel reports. The Technical Information Specialist assists each panel with searches for/provisions of relevant and available foreign technical literature. In-depth expert review of literature by panel members provides a basis for judging the quality of foreign science research activities. The preparation of assessment reports by the panels of experts is assisted to academic-quality completion by the Senior and Assistant Editor.

\section{FOREIGN APPLIED SCIENCES ASSFSSMENT CENTER}

$\begin{aligned} \text { Director } & \text { Robert R. Cronin } \\ \text { Senior Scientists } & \begin{array}{l}\text { Dr. Joel Bengston } \\ \text { Dr. Robert B. Davidson }\end{array} \\ & \text { Barbara L. McKenney } \\ \text { Senior Editor } & \text { Natalie Sluzar } \\ \text { Technical Information Specialist } & \text { Moira McGrain }\end{aligned}$

1710 Goodridge Drive, McLean, VA 22102 (703) 734-5528 


\title{
SOVIET PRECISION TIMEKEEPING RESEARCH AND TECHNOLOGY ...
}

\author{
R. F. C. Vessot \\ D. W. Allan \\ S. J. B. Crampton \\ L. S. Cutler \\ R. H. Kern \\ A. O. McCoubrey \\ J. D. White
}

August 1991

This document was prepared as an account of work sponsored by the United States government. The views and opinions of authors expressed herein do not necessarily state or reflect those of the United States government, the authors' parent institutions, or Science Applications International Corporation, and shall not be used for advertising or product endorsement.

Science Applications International Corporation

1710 Goodridge Drive, P. O. Box 1303, McLean, VA 22102

10260 Campus Point Drive, San Diego, CA 92121 
(blank) 


\section{ABSTRACT}

This report is the result of a study of Soviet progress in precision timekeeping research and timekeeping capability during the last two decades. The study was conducted by a panel of seven US scientists who have expertise in timekeeping, frequency control, time dissemination, and the direct applications of these disciplines to scientific investigation. The following topics are addressed in this report: generation of time by atomic clocks at the present level of their technology, new and emerging technologies related to atomic clocks, time and frequency transfer technology, statistical processes involving metrological applications of time and frequency, applications of precise time and frequency to scientific investigations, supporting timekeeping technology, and a comparison of Soviet research efforts with those of the United States and the West.

The number of Soviet professionals working in this field is roughly 10 times that in the United States. The Soviet Union has facilities for large-scale production of frequency standards and has concentrated its efforts on developing and producing rubidium gas cell devices (relatively compact, low-cost frequency standards of modest accuracy and stability) and atomic hydrogen masers (relatively large, high-cost standards of modest accuracy and high stability). In the West, hydrogen masers have been used exclusively in applications where extraordinarily high frequency stability is required and accuracy is of little importance. In contrast, the Soviet Union has placed an enormous emphasis on atomic hydrogen masers in an effort to develop them as accurate standards. Presently, Soviet production of hydrogen masers is at least 20 times that of the United States. These standards are deployed throughout the Soviet timing centers (six are in $\mathrm{Cuba}$ ) and are used to maintain operational time scales. Soviet work on cesium beam devices, upon which the International System of Units is defined, is lagging that of the West. The Soviet use of time and frequency as a basis for metrology and their capabilities for time and frequency dissemination and precise time transfer also lag the West. The recent Soviet deployment of the GLONASS global navigation system, which also can be used for high-precision time transfer, should substantially improve their applications of time and frequency technology. 
The quality and productivity of Soviet scientists involved in theoretical work in timekeeping are comparable to those in the West. Despite the obvious inferiority of the Soviet technical base, Soviet experimenters, by their ingenuity and talent, compete favorably with their Western rounterparts. Evolution of Soviet scientific progress into technological advances and manufacturing has been slow and only rarely ahead of the West.

In general, the Soviet Union has not been innovative, but has followed Western ideas, particularly those reported in the public domain. However, the gap is closing and there are few aspects of Western timekeeping technology that are not now fully understood, and to some extent redeveloped. The main question is in the Soviet capability to implement this technology in a cost-effective manner, a serious problem in the process of their redirection to a market economy.

Time and frequency developments in the Soviet Union have followed somewhat different paths than those in the United States; however, these developments have been motivated by comparable military and scientific needs. Soviet progress in the above topics is described chronologically to show how these developments evolved and who the principal players have been. 


\section{SOVIET PRECISION TIMEKEEPING RESEARCH AND TECHNOLOGY \\ TABLE OF CONTENTS}

Section

Page

Abstract

Table of Contents $\quad$ v

List of Figures and Tables $\quad x i$

Foreword $\quad$ xv

Executive Summary $\quad$ xvii

Chapter I ASSESSMENTS

A. Introduction I-1

B. Atomic Frequency Standards I-2

1. Cesium Beam Frequency Standards I-2

2. Rubidium Gas Cell Standards I-4

3. Hydrogen Maser Standards I-5

C. New and Emerging Frequency Standard Technologies I-7

1. Optically Pumped Standards I-8

2. Stabilized Laser Optical Standards I-9

3. Trapped and Cooled Ions I-9

4. Cryogenic Hydrogen Masers I-10

5. Cryogenic Electromechanical Resonators I-11

D. Dissemination and Time and Frequency Transfer I-12

1. Satellite Techniques I-12

2. Loran-C, Television, and High-Frequency Radio Techniques I-15

3. Meteor Trail Reflection Techniques I-17

4. Time Transfer by Transportable Hydrogen Maser
Clocks

5. Time and Frequency in Soviet Communications I-18

E. Metrology and Statistical Representations I-18

F. Relevant Science Applications in Very Long Baseline Interferometry, Reliability and Gravitation, and Quantum Mechanics

1. Soviet Very Long Baseline Astronomy Astrophysics Programs

$\mathrm{I}-23$

2. Relativity and Gravitation Studies I-25

3. Quantum Metrology I-25 


\section{TABLE OF CONTENTS}

Section

Page

Chapter I ASSESSMENTS (cont'd.)

G. Available Supporting Technology I-27

1. Quartz Crystal Resonators I-27

2. Frequency Multipliers and Synt «._sizers I-28

H. Soviet Institutions Involved in Time and Frequency Technology

Chapter II INTRODUCTION AND TUTORIAL
A. Summary
B. The Metrology of Frequency and Time: A Review of
II-1
Present Definitions of Distance and Time
II-1
C. Frequency Accuracy and Frequency Stability: Time Accuracy and Time Stability
D. Applications of Frequency Standards and the Use of the Allan Standard Deviation
II-5
E. Effects of Gravitation and Relativity
F. Atomic Clocks
II-10
G. Atomic Resonators for Atomic Clocks
II-12
H. Current Status of Atomic Clocks

Chapter III ATOMIC FREQUENCY STANDARDS

A. Summary

III-1

1. Rubidium Gas Cell Standards

III-1

2. Cesium Beam Frequency Standards

III-2

3. Hydrogen Maser Standards

III-4

B. Introduction

III-6

1. Rubidium Gas Cell Resonators

III-11

2. Cesium Beam Frequency Standards

III-14

3. Cesium Beam Resonators

III-15

C. Cesium Atomic Clocks

III-17

1. Summary

III-17

2. Discussion of Soviet Work

III-18

3. Projections for the Future

III-23

D. Rubidium Atomic Clocks

III-24

1. Summary

III-24

2. Discussion of Soviet Work

III-25

3. Projections for the Future

III-26 


\section{TABLE OF CONTENTS}

Section

Page

Chapter III ATOMIC FREQUENCY STANDARDS (cont'd.)

E. Hydrogen Maser Frequency Standards III-27

1. Introduction III-27

2. Discussion of Soviet Work III-33

3. Projections for the Future III-51

F. Key Soviet Research Personnel and Facilities III-52

CHAPTER III REFERENCES III-55

Chapter IV NEW AND EMERGING FREQUENCY STANDARD TECHNOLOGIES

A. Summary IV-1

B. Optically Pumped and Optical Standards IV-2

1. Optically Pumped Microwave Standards IV-2

2. Stabilized Laser Optical Standards IV-7

C. Trapped and Cold Particle Research IV-15

1. Trapped and Cooled Ions IV-16

2. Cooled and Trapped Neutral Atoms IV-21

3. Cryogenic Hydrugen Masers IV-27

D. Cryogenic Electromechanical Oscillators IV-30

1. Summary IV-30

2. Introduction IV-31

3. Discussion of Soviet Work IV-33

4. Projections for the Future IV-35

E. Key Soviet Research Personnel and Facilities IV-35

CHAPTER IV REFERENCES IV-39

Chapter $\mathrm{V}$ TIME AND FREQUENCY TRANSFER AND DISSEMINATION
A. Summary
$\mathrm{V}-1$
B. Introduction
$\mathrm{V}-3$
C. Dissemination Systems in the Soviet Union
$V-23$
1. Satellite Techniques
$V-23$
2. Terrestrial Techniques
$V-28$
3. Non-Traditional Techniques V-31
4. Communications Systems V-33
D. Projections for the Future
$V-35$
CHAPTER V REFERENCES
$V-39$ 


\section{TABLE OF CONTENTS}

Section

Page

Chapter VI METROLOGY AND STATISTICAL REPRESENTATIONS
A. Summary
VI-1
B. Introduction
VI-3
C. Soviet Time and Frequency Measurement Systems
VI-5
D. Projections for the Future
VI-12
CHAPTER VI REFERENCES
VI-19

Chapter VII RELEVANT SCIENCE APPLICATIONS

A. Introduction

VII-1

B. Relativity and Gravitation

VII-2

1. Summary

VII-2

2. Introduction

VII-2

3. Discussion of Soviet Work VII-8

4. Projections for the Future VII-9

C. Astrophysics and Very Long Baseline Interferometry VII-10

1. Summary

2. Introduction

VII-10

3. Discussion of Soviet Work

VII-10

4. Projections for the Future

VII-12

VII-14

D. Quantum Measurements

VII-15

1. Summary

2. Introduction

VII-15

3. Discussion of Soviet Work

VII-15

4. Projections for the Future

VII-20

VII-22

E. Key Soviet Research Personnel and Facilities

VII-22

CHAPTER VII REFERENCES

Chapter VIII AVAILABLE SUPPORTING TECHNOLOGY

A. Summary

VIII-1

B. Quartz Crystal Oscillators

VIII-2

C. Synthesizers

VIII-13

D. Frequency Multipliers

VIII-16

E. Projections for the Future

VIII-17

CHAPTER VIII REFERENCES

VIII-19 


\section{TABLE OF CONTENTS}

Section

Page

Chapter IX INSTITUTIONAL CONSIDERATIONS: THE SOVIET PRECISION TIMEKEEPING ESTABLISFIMENT

A. Introduction IX-1

B. Soviet Management of Science and Technology IX-3

C. Gosstandart (State Committee for Standards) IX-4

1. The Standardizatior. Administration IX-4

2. The Metrology Administration IX-5

D. Soviet Academy of Sciences IX-9

1. Physics Institute im. P. N. Lebedev, AS USSR, Moscow

IX-9

2. Thermal Physics Institute, Siberian Branch AS USSR, Novosibirsk

IX-10

3. Spectroscopy Institute, AS USSR, Troitsk (Kransnaya Pakhra)

IX-10

E. Universities and Polytechnical Institutes IX-11

F. Design Bureaus IX-12

G. Scientific Production Associations IX-12

CHAPTER IX REFERENCES IX-15

Appendices A. (1) Excerpts from "Performance of Soviet and US Hydrogen Masers" A-1

(2) Frequency Standards Designed for Spacecraft Use at the Leningrad Radiotechnical Scientific Institute A-7

(3) Hydrogen Masers from the Gor'kiy "Quartz" Scientific Production Association

A-17

B. About the Authors

C. Glossary of Abbreviations, Acronyms, and Specialized Terms

C-1

D. Soviet Journals Cited in Text/References

D-1

E. Soviet Research Facilities Cited in Text

E-1

F. FASAC Report Titles

F-1 
(blank) 


\section{LIST OF FIGURES AND TABLES}

Figure

Page

II.1 Progress in Clock Development

II-2

II.2 The Allan Standard Deviation II-5

II.3 Time and Range Distance Deviation from the Allan Váriance for Hydrogen Masers

II.4 Schematic of a Very Long Baseline Interferometry System II-8

II.5 Effects of Troposphere and Ionosphere on Frequency Stability II-9

II.6 Hyperfine Structure of Rubidium 87 with Zeeman Splitting II-11

II.7 Current Status of Stability of Atomic Frequency Standards II-16

III.1 Schematic of a Frequency-Lock Servo III-11

III.2 Schematic of a Rubidium Gas Cell Frequency Discriminator III-12

$\begin{array}{lll}\text { III.3 Cesium Beam Resonator } & \text { III-16 }\end{array}$

III.4 (a) Schematic of a Hydrogen Maser and (b) Energy Level Diagram of Atomic Hydrogen

III.5 Electronic System in a Hydrogen Maser III-33

III.6 Frequency Stability of Early Soviet Hydrogen Masers III-35

III.7 Frequency Stability of the Ch1-46 Hydrogen Maser III-37

III.8 Frequency Stability of the Ch1-46 Hydrogen Maser at VNIIFTRI III-43

III.9 Passive Hydrogen Maser Performance (1984-1987) III-45

III.10 Portable Clock-Sapphire III-47

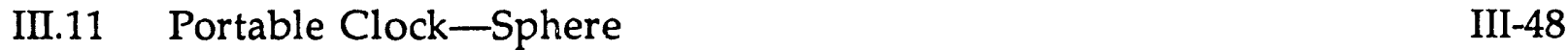




\section{LIST OF FIGURES AND TABLES}

Figure

Page

IV.1 Three-Level Optical Pumping IV-4

$\begin{array}{lll}\text { IV.2 Helium-Neon Laser } & \text { IV-8 }\end{array}$

IV.3 Methane-Stabilized Laser IV-9

$\begin{array}{ll}\text { IV.4 Schematic of an Jon Trap } & \text { IV-17 }\end{array}$

IV.5 Schematic of Mercury 199 Energy Levels $\quad$ IV-18

IV.6 Laser Recoil Force Cooling IV-19

IV.7 One-Dimensional Laser Trap IV-22

IV.8 Specular Reflection of an Atom by a Strong Light Intensity Gradient

IV.9 Hydrogen Frequency Shifts Due to Helium Collisions IV-28

V.1 Use of Frequencies According to the USSR Survey V-3

V.2 GPS Common-View Technique V-5

$\begin{array}{lll}\text { V.3 Straightforward Comparison System } & \text { V-8 }\end{array}$

V.4 Dissemination or Slave-Locked System V-9

V.5 Nominal Fractional Frequency Stability of Possible Frequency Calibration Methods

V.6 RMS Time-Deviation Error $\quad$ V-19

V.7 RMS Time Estimate Due to Random Noise V-20

V.8 UTC(SU) vs. GLONASS System Time V-26 


\section{LIST OF FIGURES AND TABLES}

Figure

Page

VII.1 VLBI System

VII-11

VII.2 Harmonic Oscillator States

VII-20

VIII.1 Doubly Rotated Quartí Cuts

VIII-4

VIII.2 Crystal Temperature Performance Close to the Turnover Temperature

VIII-7

VIII.3 Residuals vs. Temperature for the TFR Dual-Mode Oscillator SN AP7

VIII-9

VIII.4 Temperature Compensation for a Soviet Oscillator

VIII-10

VIII.5 Compensation Error: Linear Interpolation, 30 Points

VIII-11

VIII.6 Compensation Error: Quadratic Interpolation, 30 Points

VIII-12

VIII.7 Noise Increase Around the Carrier

VIII-13

VIII.8 Generic Atomic Clock

VIII-14

Table

Page

III.1 Tabulation of Western/Soviet National Cesium Standards

III-21

III.2 Comparison of GLONASS (GL) and NAVSTAR (PRN) Clock Flicker Floor

III-23

III.3 Key Soviet Research Personnel and Facilities-Atomic Frequency Standards

III-53

IV.1 Key Soviet Research Personnel and Facilities-New and Emerging Frequency Standard Technologies

IV-36

V.1 Frequency Drift in GLONASS Satellite Clocks

$\mathrm{V}-25$

VI.1 Summary of Measurement Methods

VI-6 


\section{LIST OF FIGURES AND TABLES}

Table

Page

VII.1 Key Soviet Research Personnel and Facilities-Relativity and Gravitation, Very Long Baseline Interferometry, and Quantum Mechanics

VII-23

VIII.1 Comparison of Soviet and US Oscillators VIII-12

VIII.2 Common Frequency Standards

VIII-14 


\section{FOREWORD}

This report, Soviet Precision Timekeeping Research and Technology, is one in a series of technical assessment reports produced by the Foreign Applied Sciences Assessment Center (FASAC), operated for the Federal government by Science Applications International Corporation (SAIC). These reports assess selected fields of foreign (principally Soviet) basic and applied research, evaluate and compare the foreign state of the art with that of the United States and of the West in general, and identify important trends that could lead to future applications of military, economic, or political importance. This report, like others produced by the Center, is intended to enhance US knowledge of foreign applied science activities and trends, to help reduce the risk of technology transfer, and also to provide a background for US research and development decisions. Appendix $\mathrm{F}$ is a list of titles of FASAC reports completed and in production.

This report was prepared by a panel of nationally recognized scientists and engineers who are active in precision timekeeping research. The panel membership is:

- Dr. Robert F. C. Vessot (Chairman)

- Mr. David W. Allan

- Dr. Stuart J. B. Crampton

- Dr. Leonard S. Cutler

\author{
Senior Physicist \\ Harvard-Smithsonian Center for \\ Astrophysics
}

Senior Scientist Time and Frequency Division National Institute of Standards and Technology

Director and Professor Bronfman Science Center Williams College

Distinguished Contributor Hewlett-Packard Laboratories 
- Mr. Robert H. Kern

- Dr. Arthur O. McCoubrey

- Dr. Joseph D. White
President

Kernco, Inc.

Acting Chief

Temperature and Pressure Division

National Institute of Standards and

Technology

Section Head

Acivanced Technology Section

US Naval Research Laboratory

On a part-time basis, over the period from May 1989 to January 1991, each panel member devoted a substantial amount of time toward assessing the Soviet research in precision timekeeping. The principal Soviet technical publications reviewed by the panelists are listed in Appendix D.

While most of the information for this assessment was gathered from translations of Soviet scientific journals, a great deal of insight was gained from direct personal contacts with Soviet scientists and engineers. The recent opening up of many previously inaccessible Soviet territories, such as the high-tech city of Gor'kiy, ${ }^{1}$ and the present direct access to new Soviet atomic clocks brought to US laboratories by their manufacturers, have allowed the panel to confirm the earlier conclusions reached by far less direct means.

1 The city of Gor'kiy has recently resumed its pre-1932 name, Nizhniy Novgorod. As the research for this report predates this recent change, the Gor'kiy name wili be retained throughout the report. 


\section{EXECUTIVE SUMMARY}

Precision timekeeping involves a number of disciplines. This assessment of Soviet research has concentrated on the following topics that are germane to the present state of the art:

- generation of highly stable signals by atomic clocks;

- new frequency standards: new technologies involving the control of frequency by atomic resonances;

- dissemination of time and frequency information over large distances;

- metrology: the methodology required to characterize the performance of clocks and oscillators;

- scientific applications of precise time and frequency, and fundamental quantum physical processes involved in the generation of highly stable signals;

- $\quad$ supporting technology in the Soviet Union; and

- Soviet precision timekeeping institutions involved in precision timekeeping research.

\section{A. BACKGROUND}

The fundamental basis of timekeeping is the existence of a stable oscillation to provide a regular series of events. Traditionally, this role has been fulfilled by the rotation of the Earth or by its yearly orbit about the sun. For shorter intervals, the pendulum and balance wheel of mechanical clocks and watches have long been familiar to us. The invention of the quartz crystal resonator in the 1930 s has since largely displaced these mechanical oscillators.

Since the early 1950s, quantum processes in atoms have been used to stabilize the frequency of electromagnetic signals. These processes involve transitions 
between highly deterministic energy levels characteristic of the allowed states of the nuclei and orbital electrons of specific atoms. These transitions between levels are accompanied by the emission or absorption of fixed units of energy or quanta like those perceived as spectral lines in the optical regime. Similar quantum emissions or absorptions occur at microwave frequencies and are associated with transitions between energy levels with the far smaller separations due to different states of magnetic interaction between nuclei and their orbital electrons.

Techniques for using these microwave quantum transitions in atoms, and more recently in ions, are the basis for atomic clocks. To achieve high precision measurements, the atoms must be kept isolated as much as possible from external perturbations that affect their magnetic interactions and should be allowed as much time as possible in this unperturbed condition. Inevitably, there will be some residual external perturbations that will affect the atoms' transition frequencies. The effects of these perturbations and the effects of noise processes that corrupt the measurement must both be allowed for in establishing the precision of an atomic frequency standard. The success of the design of a frequency standard depends significantly on the intended applications. Two aspects are important: frequency accuracy and frequency stability.

The concepts of accuracy and stability involve mutually exclusive processes; high levels of signals required for frequency stability over short time intervals require high rates of atomic interaction and the resultant risks of mutual interference and of reduced isolation times. Frequency accuracy demands a high degree of isolation of the quantum system from all spurious perturbing effects and a precise knowledge of the perturbations that, nevertheless, are inevitably present.

Traditional atomic clocks that have been developed in the last four decades, and now in general use, include the rubidium gas cell and cesium beam frequency discriminators, and the atomic hydrogen maser oscillators. All of these clocks operate from quantum mechanical resonances associated with the interaction between the magnetic dipole of an outer, or valence, electron and that of the atomic nucleus. Both of these interacting particles have intrinsic angular momentum, or spin, and when they are coupled together, the laws of quantum mechanics specify the existence of very distinct energy levels associated with the 
specific relative orientations of the two magnetic dipoles. Since these energy levels have differences far smaller than the energy differences associated with optical transitions, they are called hyperfine levels and are associated with microwave frequencies. In passive devices, changes between these levels can be caused by applying signals at the resonance frequencies and detecting a change in the state of the atoms by various techniques. The state changes are used in electronic servo systems to control the frequency of the applied signal so that it is centered on the quantum resonance. In active oscillators, notably the atomic hydrogen maser, the actual signal resulting from transitions induced from the upper to lower energy levels provides the required signal. Hydrogen masers can also be operated below the threshold of oscillation as extremely narrow band amplifiers and used as passive frequency discriminators to control the frequency of quartz crystal oscillators.

Today's active hydrogen maser oscillators are capable of achieving frequency stability over several hours in the 10-16 domain; on the other hand, the frequency accuracy of the hydrogen maser is rarely better than 1 part in 10-14. Over time intervals of weeks to years, cesium beam devices in national laboratories have achieved frequency stability in the $10^{-15}$ domain and have manifested frequency accuracy at comparable levels.

Because of the accuracy capabilities and widespread use and success of cesium beam frequency standards, the cesium atom has been the basis for the definition of the System International, or SI, second-of-time interval since 1967. In 1983, the SI unit of length, the meter, was defined in terms of the velocity of light. The atom now serves as clock and meterstick.

\section{B. FREQUENCY STANDARDS: GENERATION OF HIGHLY STABLE SIGNALS BY ATOMIC CLOCKS}

Early Soviet activity in atomic frcquency standards appears to have concentrated on the hydrogen maser shortly after its invention in the United States in 1959. There has been extensive Soviet development of hydrogen masers for use as primary frequency and time standards and as working standards in the timing substations located in the vast Soviet territories. With the possible exception of actively oscillating spaceborne hydrogen masers (in contrast to passive hydrogen 
masers operated as frequency discriminators), Soviet hydrogen masers are stateof-the-art devices. Less than 100 hydrogen masers are in use worldwide by Western countries, where their unbeatable frequency stability for intervals less than $10^{4}$ seconds is essential for very long baseline interferometry (VLBI) operations of radio astronomy and deep-space tracking.

In contrast to these relatively few applications thus far used in the West, the Soviets use ensembles of six or more of these devices in each timing substation for high-precision timekeeping (six Soviet hydrogen masers are known to be operating in Cuba). It appears that more use of hydrogen masers is indicated, both in the Soviet Union and in the West, the impetus being the requirements for signals having short-term frequency accuracy and frequency stability for highprecision navigation by the Soviet GLONASS and the US Global Positioning System (GPS) and for high-precision data links between high-speed computers.

Soviet progress in cesium beam devices has concentrated mainly in two areas: primary laboratory standards, and spaceborne frequency standards for GLONASS. The Soviet decision to concentrate on hydrogen masers as frequency standards capable of being both accurate and stable was sidetracked by the definition of the SI second based on cesium. The Soviet primary standards laboratory at the Physical Technical and Radio Engineering Measurements Institute (VNIIFTRI) was forced to catch up with the West by copying Western designs.

While information on Soviet spaceborne cesium beam devices is sparse, we know that they are being used in GLONASS and that their performance is approaching that of US cesium standards in GPS spacecraft.

For general-purpose mobile use on various types of vehicles, the Soviet Union has relied on rubidium gas cell devices, the best of which are comparable in performance to those made in the West. These are mass produced, along with hydrogen masers, at the "Quartz" Scientific Production Association (NPO) in Gor'kiy, where production figures for rubidium are alleged to be about 500 per year. For hydrogen masers, we estimate production rates to be about 30 to 40 per year. Recently (December 1990), we have learned of the manufacture of spaceborne rubidium, cesium, and passive hydrogen standards at the Leningrad Radiotechnical Scientific Research Institute. As yet, there is little information 
about these devices, other than some outline drawings, and performance, weight, and power specifications (shown in Appendix A).

In November-December 1990, a significant manifestation of glasnost' occurred in the visit to the Smithsonian Astrophysical Observatory by members of the Gor'kiy "Quartz" NPO along with two of their highly advanced hydrogen masers. One of these masers, designated as Ch1-75, is truly a state-of-the-art active oscillator. The other maser, the Ch1-76, is a very compact hydrogen maser that operates as a passive frequency discriminator. Both of these masers were run for about 30 days, and their frequency stability was evaluated both in the short term and in the long term. The performance of the Ch1-75 maser was found to be fully comparable in the short and long term to the best US-made hydrogen masers. The Ch1-76 passive maser performance also was found to be excellent. Soviet researchers have made a breakthrough in hydrogen maser technology by developing a new surface coating for the hydrogen storage volume that allows an eight- to tenfold improvement in frequency stability and accuracy of frequency reproduction. Use of this coating in US-made hydrogen masers would significantly improve their performance.

A study of the available literature on hydrogen masers reveals that, until the early 1980s, the Soviet efforts were about three years behind US efforts and Soviet researchers freely copied US progress that was available to them through US publications. This is far less true for cesium and rubidium devices, since these are industrially produced in the United States and subject to proprietary restrictions. Nevertheless, despite trade restrictions on frequency standards, many highquality US-made frequency standards have found their way into the Eastern Bloc to be used in standards laboratories and often copied. Thus far, it appears that the Soviet success with field-operable cesium beam frequency standards has been modest compared with rubidium and hydrogen, largely because of the very demanding materials control, vacuum cleanliness, and critical processing involved in cesium beam devices.

\section{NEW FREQUENCY STANDARDS}

New species of clocks that promise to extend the frequency accuracy and stability of clocks are under development. These involve the use of stored atoms 
and ions that, by the use of various electromagnetic traps, laser traps, and cooling techniques, can be levitated and immobilized for hours and even longer. The high degree of isolation made possible by these techniques permits an extremely high degree of frequency discrimination. Precise frequency controllable signals of very narrow spectral linewidth are required to excite these resonant transitions so as to remain within the frequency width of these discriminators.

New and emerging frequency standard technologies leading to new and more exotic frequency standards have, for some time, been under development in the Soviet Union. These are based on the use of stabilized lasers with the goal of producing a combined length-time standard operating at optical frequencies. This ambitious effort has been thwarted by the difficulty of constructing a chain of frequency multipliers to coherently relate the optical signal used to define length to the microwave domain signal to define frequency. In contrast to this laser work, advanced techniques in the West, predominantly involving trapped ions and atoms that are cooled by interaction with laser light tuned to resonant optical transitions, are clearly of interest to Soviet researchers but, as yet, they have shown no significant progress.

The use of cryogenic techniques for operating superconducting resonators of enormously high $Q$ is well understood in the Soviet Union, and pioneering work on internally reflected "whispering-gallery modes" in dielectric resonators has been done at Moscow State University. These techniques are important for the generation of spectrally pure signals for use in standards using trapped and cooled ions and atoms. There is no evidence of work on cryogenically cooled hydrogen masers that operate at temperatures near $0.5 \mathrm{~K}$, which could offer substantial improvements in botk long- and short-term frequency stability.

\section{DISSEMINATION OF TIME AND FREQUENCY}

In the dissemination of time and frequency, the Soviet Union relies on the same basic techniques as the West, with one notable exception involving reflection of signals from meteor trails. As in the West, the synchronization of communications systems is an important issue. 
Use of the GLONASS navigation system, which is similar to the US Global Positioning System, involves the same technology for timekeeping. The principal difference between the two systems is the use of 12-hour orbits in GPS in contrast to the 11-hour orbits used in GLONASS. So far, the frequency stability of GLONASS clocks is somewhat inferior to those of GPS, and the difference is rapidly diminishing, even as GPS clocks are improved. There is speculation that GLONASS satellites can communicate with each other and determine their separation distances. If so, the GLONASS capability to determine precise satellite position and velocity is greatly improved over that of GPS, and the control of GLONASS can be done from one Earth station, rather than the several stations required for GPS. There is no indication of a Soviet capability to selectively degrade the precision of timekeeping and navigation for general users, reserving the high-precision capability for selected users as in GPS. The assurance of continued GLONASS high-precision capability is attractive to manufacturers of navigational equipment and could lead to a worldwide preference for GLONASS. Navigational equipment operating with both GLONASS and GPS receiving capability likely will appear in the marketplace soon.

The Soviet Union uses most of the traditional terrestrial time transfer techniques in the VLF, LF, MF and HF standard broadcast bands and, in the MF and HF bands, is known to use frequencies somewhat outside the approved bands to reduce interference. Extensive use is made of TV techniques, both terrestrially and by satellite. As in the United States, the majority of Soviet working frequency references are rubidium gas cell devices, and these are often used as portable clocks.

In a significant advance over US practice, Soviet researchers have developed hydrogen masers for use as portable clocks. These have been highly effective for time synchronization trips between Moscow and the Paris Observatory and several times to Cuba.

Soviet researchers are exclusive in their use of reflections off the ionization from meteor trails to reflect signals back and forth between stations. This technique is effective and meets their geographical requirements. Further use of the less precise capabilities of Loran- $C$ and other low-frequency techniques will continue as the Soviet facilities are expanded for navigational use. 
At present, time synchronization of Soviet communication systems is inferior to that of the West, but it is expected to improve. Expanded use of GLONASS for timing purposes can be expected, along with the Soviet adoption of high-precision techniques developed in the United States with GPS.

\section{E. METROLOGY}

Generally, Soviet frequency and time measurement techniques follow Western practices, with somewhat inferior stability accuracy and measurement noise. Some Western innovations are well understood but have yet to be implemented. For scientific applications, the Soviet Union is generally up to date with the West in applications of clocks and frequency standards of the highest stability. Soviet researchers are active in testing theories of relativity and gravitation and in very long baseline interferometry applications to radioastronomy. Both of these experimental fields require the best frequency standards.

With the accuracy and stability available from atomic frequency standards at such high levels, any comparison of time or frequency between clocks in relative motion and/or at different gravitational potentials must include the effects of general relativity. For example, a correction factor of $1 \times 10^{-13}$ must be applied between clocks at the mile-high city of Denver and the Atlantic/Pacific coast; the Denver clock will appear to run faster. Larger corrections are included in the engineering design of spaceborne clocks for navigation systems.

\section{F. SCIENTIFIC APPLICATIONS}

Relativistic gravitation theory plays a major role in timekeeping, both in the microscopic sense, when considering rapidly moving atoms and ions, and in the vastly large scale involving time and frequency globally, and in outer space. Oscillators of the highest stability are needed to test the theory, and, in turn, the theory is vital for understanding the behavior of oscillators. The Soviet Union is at the forefront of this work, particularly in the corrections applicable to GLONASS and GPS. 
In the development of theoretical relativity and gravitation, a new alternative theory has been advanced by a Soviet author that, while mathematically consistent, differs from the fundamental approach advanced by Einstein. Most experts, including some Soviet, disagree with the new theory and generally it has gained lin acceptance.

Quantum mechanics is another field of science relevant to clocks and frequency standards, and was originally pioneered by scientists in Western Europe and the United Kingdom. Soviet physicists, particularly in recent years, have made strong contributions to the theory in applications of quantum processes for measurements of time and distance on very small scales. There has been successful cooperation between US and Soviet theorists in this work, resulting in jointly published books and technical articles. This cooperation continues to make substantial contributions to the understanding of quantum processes.

The very long baseline interferometry techniques of radio astronomy require frequency standards of very high stability for intervals from minutes to hours; these requirements are met by the use of atomic hydrogen masers. This technique provides the highest angular resolution of any istronomical measurement process, a resolution capable of measuring an angular displacement equivalent to reading a newspaper in Los Angeles while standing in Boston. There are many applications related to VLBI in other fields, such as satellite tracking and navigation. Operating in reverse, using celestial sources as radio beacons, VLBI is used for geological measurement of continental drift. The Soviet Union is active in VLBI research and currently has a program to operate VLBI radioscopes in space, where there are no baseline distance limitations and no frequency limitations due to the atmosphere and ionosphere. This program, called "Radioastron," will eventually require active hydrogen masers. At present, the program involves substantial international cooperation, including participation from the United States. While Soviet astrc -omers, astrophysicists, and engineers are highly competent, the Soviet technological capability for recording data and performing the correlation processes necessary for VLBI is inferior to that of the United States. As yet, little is known about the quality of Soviet radio telescope antennas, particularly at millimeter wavelengths. 


\section{G. SUPPORTING TECHNOLOGY}

Atomic frequency standards require electronic circuitry that includes quartz crystal oscillators, frequency multipliers and synthesizers, laser sources, and lownoise electronic amplifiers. Low-noise, high-stability quartz crystal oscillators are used to generate signals for causing resonant quantum transitions in atomic clocks. The Soviet development of quartz oscillators is significantly behind that of the United States, despite a clear understanding of modern concepts. In frequency multipliers and frequency synthesizers, the Soviet technology appears to be on a par with that of the United States. In laser light sources, the Soviet activity shows considerable competence in developing systems for optical state selection in atomic frequency and laser frequency standards. High-quality quartz for crystal oscillators is abundantly available in the Soviet Union, but this seems to be the only advantage the Soviets enjoy. While Soviet researchers understand that the use of modern crystallographic orientations in the quartz for lowtemperature coefficients reduces acceleration sensitivity, there is no evidence of their use in the Soviet literature. On the other hand, publications on sophisticaied dual-mode oscillation techniques with computer control, once classified in the United States, have appeared in the published Soviet literature. The Soviet electronic techniques clearly are far ahead of their quartz cutting and grinding techniques.

Computer-operated digital synthesizer techniques using numerically controlled digital-to-analog converters are reported in the Soviet patent literature, but, thus far, do not appear to be used in the best Soviet frequenc"' and time standards or timekeeping systems.

Frequency multipliers are an essential part of atomic frequency standards to obtain signals in the microwave region. Here, Soviet workers have followed the usual practices for atomic standards. Research on superconductive point-contact devices for multiplying and mixing signals to frequencies as high as $10^{13} \mathrm{~Hz}$ has been reported. Experimental work on metal-oxide-metal diodes for harmonic generation to the submillimeter and infrared region has also been reported. These developments have followed similar US work. 


\section{H. SOVIET PRECISION TIMEKEEPING INSTITUTIONS}

The organization of the Soviet precision timekeeping establishment differs considerably from that in the United States. While it is not clear how this organization will evolve, the existing order reflects the controlling role of centralized planning. Modern precision timekeeping began in the universities, with parallel advances reflected in thie 1964 Nobel Prize jointly awarded to Charles Townes of the United States and to Nikolay Basov and Aleksandr Prokhorov of the Soviet Union. In the mid-1950s, US scientists became involved in practical and cooperative developments with US industry leading to cesium beam, rubidium gas cell, and, in 1962, hydrogen maser frequency standards. In the Soviet Union, the first practical quantum standard was in the ammonia maser, begun in 1959, followed by the hydrogen maser in 1967, and the cesium beam standard in 1976 .

The Soviet Union has abundant intellectual resources to do any type of work related to time and frequency. However, knowing how to, and actually doing the work, are quite different concepts. The wide diversity of time and frequency activities requires access to support from a broad technological base. For the most part, Soviet researchers have the technology, but their economic system makes access to it extremely cumbersome and, in some cases, nearly impossible. Another factor that may be important in comparing US and Soviet work is the more rigid Soviet academic structure, where institutes of higher learning tend to be more formal and compartmented among scientists, engineers, and technicians. Entrepreneurial scientists and engineers with a flair for "hands-on" experimental work are "rare birds" in the Soviet Union, and if they do somehow appear, their flights of discovery are surely far more circumscribed by their lack of experimental hardware and specialty shops, such as glass blowers and metallurgical annealing. There is nothing in the Soviet Union approaching the availability of electronics components such as from a Radio Shack or the myriad electronic suppliers available in the United States. Budding young Soviet scientists or engineers will rarely have available the "do-it-yourself" facilities of their North American counterparts with cellar workshops and some type of technical hobbies at which to tinker.

There are clear, fundamental differences between the United States and the Soviet Union in making practical progress in precision timekeeping. Academic 
and industrial scientists in the United States communicate freely, and financial incentives are available for industrial collaboration. US Government agencies such as the Department of Defense and the National Aeronautics and Space Administration (NASA) encourage such activity. In the Soviet system, there is little evidence of such incentives, and bureaucrats quite removed from sciense and opportunities for applications seem to control scientific and technical progress. 


\section{CHAPTER I}

ASSESSMENTS

\section{A. INTRODUCTION}

The Soviet Union clearly has the intellectual talent to master any aspect of time and frequency work. Questions related to time attract the attention of the best and brightest of Soviet scientists. Soviet researchers are as knowledgeable in most areas of timekeeping as their Western counterparts. Kip S. Thorne, a professor at the California Institute of Technology, who is intimately familiar with Soviet research, comments in his foreword to the English translation of a book by Braginskiy, Panov, and Mitrofanov entitled Systems with Small Dissipation (University of Chicago Press, 1985), which deals with questions related to the physical basis of the highly stable oscillations required for timekeeping:

Their ingenuity and experimental talent have enabled [Soviet researchers] to compete with experimenters in the West and, despite the inferiority of the Soviet technological base, to win that competition more often than not.

Because of its inferior technological base, the Soviet Union suffers significantly, especially in the area of computers, and in other areas that are dependent upon state-of-the-art computing technology. At the outset of this study, it appeared that this low technological base could also affect Soviet timekeeping research; however, based on the literature reviewed, it appears that the Soviet Union has access to most of the necessary materials to make good progress. Time and frequency is an area where progress does not demand access to exotic new materials that might not be legitimately available in the Soviet Union.

While there is abundant evidence of Soviet scientific prowess both in theory and experimental researsh, very few technological advances not previously known in the United Sitates have been made. On the other hand, nearly all of the important technological advances made in the United States seem to be understood and used in the Soviet Union. 


\section{B. ATOMIC FREQUENCY STANDARDS}

Soviet research and development activities on atomic frequency standards have focused chiefly on the hydrogen maser, which was clearly intended, at some time (probably the early 1960s), both to define the national standard of time and frequency and to serve as a type of working standard in various laboratories and in the network of time-synchronized stations throughout the Soviet Union. With the 1967 adoption by the System International (SI) of the unit of time based on the cesium atom, it became necessary for Soviet researchers to develop a national standard of time and frequency based on cesium. There are only two legally certified cesium standards capable of manifesting the definition the second in the Soviet Union. They are located in the State Time and Frequency Service's main laboratory at the Physical-Technical and Radio Engineering Measurements All-Union Scientific Research Institute near Moscow (commonly referred to as VNIIFTRI). Two other cesium standards are under investigation. However, Soviet research has continued to improve both the accuracy of frequency reproduction and the stability of hydrogen masers. Hydrogen masers continue to be the backbone of the operational systems in the Soviet time and frequency network, operating as secondary standards in Uzhgorod, Khar'kov, Novosibirsk, Irkutsk, and Khabarovsk.

Research and development of working standards based on cesium and rubidium appear to be centered in the "Quartz" Scientific Production Association (NPO), an industrial complex in Gor'kiy, ${ }^{1}$ and in the Leningrad Radiotechnical Scientific Research Institute, where military and spaceborne equipment is manufactured. There appears to be some competition between the Gor'kiy group and the VNIIFTRI group in the development of hydrogen masers.

\section{Cesium Beam Frequency Standards}

The published information on Soviet rubidium and cesium devices is spa 'e. However, it is clear that Soviet work on primary cesium beam standards

1 The city of Gor'kiy has recently resumed its pre-1932 name, Nizhniy Novgorod. As the research for this report predates this recent change, the Gor'kiy name will be retained throughout the report. 
lags that of the West, most notably the United States, (West) Germany, and Canada. Reports of Soviet work on primary cesium devicr first appeared about eight years after the 1967 SI adoption of the cesium 133 atom to define the second.

Based on the progress of the Soviet primary standards at VNIIFTRI, as mentioned in the 1989 Soviet report to the Consultative Committee for the Definition of the Second (CCDS) and other publications, it appears that these devices have closely followed Western designs with very little innovation. The two legally certified Soviet cesium beam standards have performed well, with a relative frequency difference of less than $5 \times 10^{-14}$ from 1985 through 1988. Their accuracy is reported to be approximately $1 \times 10^{-13}$, which is a factor of 10 below the accuracy of Western standards. Many of the cesium beam working standards operating in the Soviet Union appear to be of Western manufacture (chiefly Hewlett-Packard 5061A devices). Currently, very little is known about the Soviet manufacture of cesium beam devices for laboratory and field applications, although one advertising brochure dated 1974 was found.

There has been considerable Soviet progress in space-qualified cesium beam standards for the GLONASS system, as indicated by the recent revelation by Soviet researchers at the European Time and Frequency Congress in March 1990.2 It is not yet known whether the actual cesium beam tubes are of Soviet manufacture, or are purchased abroad. The performance of the most recently launched clocks in the GLONASS system is characteristic of cesium devices. Whether or not earlier, less frequency-stable spaceborne GLONASS clocks were based on cesium beam tubes with poorly functioning electrorics, or on rubidium gas cell devices is not known as of November 1990. It is not clear how the Soviet Union will proceed with the domestic manufacture of cesium beam standards. Recently, some brochures have appeared from the Leningrad Radiotechnical Scientific Research Institute advertising spaceborne cesium frequency standards.

2 N. E. Ivanov, V. A. Salishchev, and A. O. Kupriyanov, "Some Aspects of Space Navigation System GLONASS: Application in the Interest of Time and Frequency Determination," Proc. 4th European Frequency and Time Forum, 13-15 March 1990 (Neuchâtel, Switzerland). 


\section{Rubidium Gas Cell Frequency Standards}

Rubidium gas cell frequency standards are being manufactured on a large scale in the Soviet Union in Gor'kiy and in Leningrad. There are numerous references in the literature to rubidium devices and their calibration using hydrogen masers. A 1974 prospectus describes the Soviet Ch1-48 device, which appears to have been manufactured in considerable quantities. It is a product of modest but useful stability which was offered for sale in 1974 for $\$ 10,000$.

Optical pumping technology for rubidium gas cell standards clearly is well advanced in the Soviet Union. These are mentioned as backup oscillators in a prospectus for $\mathrm{Ch} 0-101 \mathrm{~b}$ systems, which operate four hydrogen maser frequency standards. Reference is also made to the use of HP-5065A rubidium standards, as well as standards manufactured by Rhode \& Schwartz in (West) Germany.

The technology for the production of rubidium devices was first reported in the Soviet Union in 1983, some 10 years after it was reported in (West) Germany. Good original work is also reported, and it is clear that the Soviet technology is now well advanced with the 1984 production of Ch1-69 devices which are consicierable improvements over contemporary HP-5065A and Efratom XSRM devices. The recently developed Ch1-74 mobile unit resembles the HP-5065A and it appears to be competitive with any existing rubidium gas cell products available in the West.

Soviet progress on rubidium gas cell devices is accelerating, and it reflects ongoing efforts by an impressive group of scientists and engineers. Soviet rubidium standards appear to be competitive in performance with most Western products and they may well appear in the marketplace in direct competition with Western products. Dr. N. A. Demidov, who visited the Smithsonian Astrophysical Observatory (SAO) in late September 1990, reported that the production rate of rubidium gas cell frequency standards at the Gor'kiy "Quartz" NPO is about 500 per year. Four types of rubidium gas cell frequency standards for space applications are offered for sale by the Leningrad Radiotechnical Scientific Research Institute which have specifications that are suitable for a wide range of applications. As the Soviet Union changes to a market-oriented economy, these frequency standards undoubtedly will be offered for sale worldwide. 


\section{Hydrogen Maser Standards}

Based on the quantity and quality of the available literature, there is no question that the most energetic and productive Soviet work on frequency standards has been devoted to atomic hydrogen masers. Basov and Prokhorov, who shared the 1964 Nobel Prize with Townes of the United States for the invention of the maser/laser, must have lent considerable prestige to this device in the Soviet Union.

The work of Ramsey and Kleppner, inventors of the hydrogen maser in 1960, was closely replicated by Basov and reported in great detail in 1965. The 1965 report cites Basov as well as others who continue to conduct competent work both at Gor'kiy and at VNIIFTRI. At some point in time, Gor'kiy became the center for industrial research, development, and manufacture of frequency control equipment, vile VNIIFTRI concentrated on timekeeping operations as well as research and development on atomic standards. Military and space related hydrogen maser work is reported at the Leningrad Radiotechnical Scientific Research Institute. This institute advertises a compact hydrogen maser frequency standard which has modest stability and appears to operate as a passive device rather than as an active oscillator.

Early Soviet hydrogen maser work in the late 1960s and early 1970s was hampered by a lack of critical materials such as metals for magnetic shielding and up-to-date (by Western standards) test equipment and electronic components. Soviet progress was continued by very competent scientists and engineers using ingenious solutions to the problems they encountered.

In contrast to the West, there has been a high level Soviet effort to promote frequency accuracy and stability in hydrogen maser development. The Soviet Union has expended considerably more effort than the West on understanding the hydrogen collisional effects in the storage volume and on improving the predictability of the output frequency of the maser for use as a priniary reference.

The ability to store atoms in a relatively unperturbed condition by confining them in a vessel whose walls are coated with special material is the fundamental 
attribute of the maser as a highly stable oscillator. The most serious problem with the use of the maser as an accurate frequency standard is the uncertainty of the wall collisional frequency shift. In the West, where hydrogen masers are used mainly for their high stability, wall coating work has been almost completely neglected. Soviet work on fluorocarbon wall coating materials is far ahead of any in the West, and worth learning more about.

Otherwise, the Soviets have closely followed the technical developments reported on by Western researchers, and, when useful, have elaborated on them. The highly successful Ch1-75 devices presently offered for sale include such features as the use of sorption pumps to scavenge hydrogen (and requiring no power to operate), solid metallic hydrides as a source for hydrogen, the use of very low expansion glass-ceramics for the cavity resonator, and quasi-kinematic mounting structures to negate thermal strain from differences in thermal expansion between the cavity and its mounting structure-ideas which were developed for the 1976 SAO/NASA space test of general relativity. ${ }^{3}$

In the last 10 years, Soviet progress appears to have accelerated with the development of sophisticated electronic systems, including digital synthesizers, built-in comparison systems, and automatic tuners. Soviet scientists have developed both passive and active masers that are in quantity production and for sale; they have also produced small hydrogen masers for use in traveling clocks.

Soviet hydrogen masers are as advanced as any of those in the West and, in storage bulb coatings, they are ahead of the West. The Soviet hydrogen maser production capability is many times greater than that of the West; as many as 20 masers reportedly are built at one time. While their performance specifications are very good, the quality and reliability of Soviet hydrogen masers have yet to be assessed.

The question of poor reliability of hydrogen maser operation is mentioned in the 1989 Soviet report to the CCDS, but no failure modes are identified other

3 R. F. C. Vessot, M. W. Levine, E. M. Mattison, E. L. Blomberg, T. E. Hoffmann, G. U. Nystrom, B. F. Farrell, R. Decher, P. B. Eby, C. R. Baugher, J. W. Watts, D. L. Teuber and F. D. Wills, "Tests of Relativistic Gravitation with a Spaceborne Hydrogen Maser," Phys. Rev. Lett., 45, 12(1980), 2081-2084. 
than those of occasional contamination of the wall coatings and premature failures of their large ion pumps.

The future for hydrogen masers in the Soviet Union appears to be good. Soviet engineers and scientists working on hydrogen masers are as competent as those in the West and they are far more numerous. Soviet researchers know what needs to be done, and they are actively developing hydrogen masers. They are leading the United States in some respects. The latest report from the European Time and Frequency Forum in Neuchâtel (March 1989) indicates that hydrogen masers will likely be used as spaceborne clocks in the GLONASS system.

There are strong indications that the Soviet Union will continue to produce large numbers of hydrogen masers and offer them for sale. The Ch1-75 maser is a highly successful, well-designed instrument that is now in production at the Gor'kiy "Quartz" NPO; approximately 15 of these devices are being produced and undoubtedly will be offered to the world marketplace.

\section{NEW AND EMERGING FREQUENCY STANDARD TECHNOLOGIES}

Areas of research and development that are important to the future of highly stable oscillators and timekeeping include:

- the use of optical frequencies in laser standards operating at optical frequencies, and for the optical pumping of frequency standards where light is used to change the quantum states of atoms;

- the trapping and cooling of ions in radio frequency and DC magnetic traps; and

- the use of cryogenic hydrogen masers and cryogenically cooled electromechanical resonators.

The use of laser light to interact with cesium beams instead of existing simple but inefficient magnetic state selecting techniques will greatly improve these types of standards. Optical pumping with laser light will continue to play a 
strong role in the development of standards using trapped ions and atoms. Cryogenic hydrogen macers offer the possibility of producing signals with stability at the 10-18 level. Cryogenically cooled dielectric resonators also offer possibilities of producing flywheel oscillators with the exceptionally good spectral purity needed in the new generation of atomic standards.

There is significant but uneven Soviet progress in all of these areas, with more efforts on theoretical rather than experimental work. Soviet researchers in this area have concentrated on laser frequency standards with some success, and have played a significant role in the development of methods to collimate and deflect atomic beams using laser light. While they are leaders in the development of cryogenically cooled single-crystal sapphire microwave resonators, they are lagging in the use of laser pumping in cesium and rubidium standards, and in experiments involving trapping of ion and neutral atoms. No work on cryogenic hydrogen masers is reported.

\section{Optically Pumped Standards}

Optically pumped techniques with lasers to operate cesium beam and rubidium gas cell devices are progressing in the Soviet Union. However, access to good solid-state diode lasers may be a problem. It is not clear if the Soviet production of laser diodes is sufficient over an adequate range of wavelengths to support their work on frequency standards. There are some indications from discussions with Soviet scientists that research on microwave-to-optical frequency multiplication has been sidetracked. Much of the Soviet work on optical pumping appears to be theoretical rather than experimental, and except for the usual techniques for rubidium gas cell optical pumping, the main work currently in progress is on cesium beam standards at VNIIFTRI. With the exception of theoretical work, the Soviet Union is lagging the United States and other Western nations in optical techniques related to atomic frequency standards.

The Soviet ability to produce good injection lasers for fiber-optic telecommunications is expected to improve and should benefit their efforts in optically pumped frequency standards. This could be important for Soviet navigation and communication. 


\section{Stabilized Laser Optical Standards}

Highly stabilized lasers using saturated absorption of methane and other molecules have been developed successfully at the Thermal Physics Institute in Novosibirsk. The Soviet Union leads the world in the application of spectroscopic techniques to obtain extremely narrow resonances for optical standards. Considerable work has been performed to develop phase-coherent links from optical to microwave frequencies to produce a practical time-length standard; however, these microwave-to-optical systems are said to be unstable for time intervals longer than about 100 seconds. The Soviet Union is competing well at the cutting edge of the necessary technologies for eventual development of successful laser frequency standards.

Soviet scientists appear to be betting on stabilized lasers as the best technology for the next generation of frequency standards, instead of working on stored and cooled ions and atoms, as in the West. The current prospects for a practical optical standard are not good; a breakthrough is needed to obtain good stability at much longer averaging times than 100 seconds, and to achieve similar improvement in synthesizing optical frequencies from microwave frequencies.

\section{Trapped and Cooled Ions}

In recent years, precision spectroscopy with stored ions has been the most promising method for improving accuracy and long-term frequency stability. Prototype mercury ion standards are now performing as well as, or better than, hydrogen masers at the US Naval Observatory for intervals longer than about $10^{4}$ seconds, and innovations in laser cooling of single ions have the potential for a frequency accuracy of $10^{-18}$ and a frequency stability even better than this for averaging times of a day and longer. New US devilopments on trapped and cooled ions have offered new possibilities. While there is significant Soviet theoretical work in this area, no experimental work was found in the published literature.

Soviet researchers have made significant theoretical advances in cooling and trapping of neutral atoms and are working on applications of laser cooling to improve optical frequency standards and cesium beam standards. Experiments 
in manipulating atoms by laser light are being conducted by two highly prestigious groups, one led by V. S. Letokhov at the Spectroscopy Institute, and the other, more recently, led by A. M. Akul'shin at the Lebedev Physics Institute. While rnany successful new developments are underway in the United States, there is no evidence of Soviet activity in trapping laser-cooled atoms, whose long storage times and small Doppler shifts can substantially improve spectroscopic work and frequency standards.

The Letokhov group is among the world leaders in cooling and trapping atoms using laser light. The Akulshin group has concentrated its work on cesium beam collimation and slowing. This work will likely be applied to atomic beam frequency standards, and significant results can be expected. It is possible that these techniques will also be applied to optical frequency standards. Thus far, there is no sign of Soviet work on cooling and trapping atomic hydrogen, which requires more sophisticated lasers, or on laser cooling in conjunction with electrode traps.

\section{Cryogenic Hydrogen Masers}

The cryogenic hydrogen maser grew out of research on spin polarized gaseous atomic hydrogen, a field in which Soviet researchers have made strong contributions to theory. The capability of confining state selected atomic hydrogen at low temperatures has many attractive features. The interatomic collisional cross section that limits the oscillation $\mathrm{Q}$ is greatly reduced from its room temperature value and the velocity of the atoms is also reduced; the net result is a thousandfold (or greater) improvement in power output or, alternatively, substantial improvement in oscillation $Q$. The thermal noise in competition with the maser signal is also redured, and low temperature advantages in mechanical stability and the use of superconductive magnetic shields make a tempting package. Operation of cryogenic hydrogen masers has been achieved in the United States and Canada using storage volumes coated with frozen carbon tetrafluoride at $60^{\circ} \mathrm{K}$, with frozen neon at $10^{\circ} \mathrm{K}$, and with superfluid helium at $0.5^{\circ} \mathrm{K}$.

Although Soviet scientists are deeply involved in hydrogen maser research, there is no apparent activity on cryogenic hydrogen masers. 


\section{Cryogenic Electromechanical Resonators}

Frequency controllable high-stability oscillators and, in particular, conventional quartz crystal controlled oscillators are important to the operation of atomic frequency standards because of their ability to serve as "flywheel oscillators" that are either phase locked or frequency locked to atomic resonances. The development of new types of standards involving trapped ions or atoms will require controllable oscillators that are far more stable than any now available. Cryogenic resonators are strong candidates for fulfilling these requirements. Soviet scientists have made some of the most important advances in these cryogenic devices, both in experiment and in theory. Three types of resonators have been studied: (i) superconductively coated solid dielectric resonators, (ii) hollow cavities or metallic superconductors, and (iii) cavities of dielectric material without conductive coatings using total internal reflection of the microwave energy within the material (the "whispering-gallery" mode). At low temperatures, the losses in some dielectric materials such as sapphire can be exceedingly small, and these materials can also have excellent mechanical stability and thermal properties. Metallic superconducting cavity resonators at microwave frequencies have achieved resonance $Q s$ as high as $10^{11}$. However, the resonance frequency of these devices is subject to a number of mechanical stress effects and power level variations. The quality and purity of sapphire crystals is also an important factor in the low-temperature behavior of dielectric resonators.

Soviet physicists are world leaders in the theory of cryogenic electromechani-

cal resonators. Researchers at Moscow State University have made significant experimental demonstrations of high $Q$ apparatus in an ongoing program of frequency standard development. Continued successful competition with the United States will likely depend on the availability of high purity materials. The United States presently has some advantage in producing these materials. Soviet improvements in resonator $Q$, and the development of a working frequency standard at $10^{\circ} \mathrm{K}$ or lower based on a sapphire ring "whispering-gallery" mode resonator, would be landmark achievements. 


\section{DISSEMINATION AND TIME AND FREQUENCY TRANSFER}

Time and frequency transfer can be achieved in two ways: (i) by electromagnetic signals, and (ii) by transporting a clock or frequency standard (there are also systems that combine both methods). For precise transfer using electromagnetic signals, the propagation delay must be accounted for, and the random noise caused by the propagation medium also must be understood. In the transport of a clock, its time and frequency instabilities during the period of travel must be accounted for.

In the last four decades, timekeeping precision has improved from the millisecond $\left(10^{-3}\right)$ to the picosecond (10-12) level, and the frequency stability of oscillators used in clocks has advanced from the nanosecond $\left(10^{-9}\right)$ to the $10^{-16}$ level. The ability to synchronize clocks over large distances has also substantially improved.

\section{Satellite Techniques}

To date, the most precise operational means of long-distance time and frequency comparison is by the simultaneous observation at two or more stations of time signals from orbiting clocks aboard the US Global Positioning System (GPS) or the Soviet GLONASS system. In this way, the inaccuracies of the orbiting clock are common to all receivers monitoring a satellite signal at the same time, and if the position of the satellite is known so that the differences in the path delays to the stations can be determined, time synchronization at the 10-nanosecond level can be achieved. At this level of accuracy, relativistic corrections are necessary (this is discussed briefly in Section II.E). Timing centers using the above technique have achieved this level of accuracy with GPS over the last several years. This technique was first applied between the Soviet Union and the West using GLONASS in 1990. Thus far, the accuracies appear to be at the 80nan-second level.

Another method involving space technology is the use of communication satellites for time transfer. In this technique, time transfer from station $A$ to station $B$ is accomplished by having station $A$ transmit to the satellite with both stations $A$ and $B$ receiving station $A$ 's signal and recording its time of arrival 
with reference to their respective clocks. In this way, station A can measure the time delay to and from the satellite. Dividing this delay by two gives the propagation time of the signal from station A to the satellite. During this process, station $B$ also sends time signals to the satellite and measures the time of its return to determine the propagation delay from station $B$ to the satellite. The total delay in the path from station A to station B via the satellite is accounted for by adding the propagation times from station $A$ to the satellite and back to station B. When performed simultaneously, the path delays are nearly identical and each station has the necessary data to correct for propagation. One of the sources of inaccuracy is that communication satellite transmitter-receiver systems have some time-delay variability. This is also true for ground station transmitting and receiving equipment, which, thus far, have not been specifically designed for time comparison applications. Since the amount of information in the time signals required to perform this type of synchronization is minimal, very little channel capacity is required, and timing can be done with no interference with normal TV operations. Studies are continuing on the reliability and precision of this two-way technique. A two-way system has been operating for about two years in the United States between the National Institute of Standards and Technology (NIST) in Boulder, Colorado, and the US Naval Observatory.

Both the GPS/GLONASS common-view time comparison technique and the communication satellite technique are capable of comparing oscillators having stabilities of a few parts in $10^{14}$ in one day's averaging time. The Soviet Union has expressed a strong interest in cooperative satellite time and frequency transfer operations. However, there are problems with the widespread use of GPS signals for high-precision time comparison. Because of the deliberate reduction in the timing accuracy of the GPS satellite signals for ordinary users by the operation of the selective availability system (through which only the receiving stations who are privy to the high-stability data codes may obtain full precision), the use of selective availability is likely to cause a worldwide shift toward the use of GLONASS. The Soviet Union is already encouraging Western manufacturers to produce GLONASS receivers, or to include GLONASS capability in GPS receiving systems. 
Because the GPS and GLONASS systems are based on receiving capability only, the propagation distance is accounted for by knowledge of the positions of the receiver and the spacecraft. Although path delays from the troposphere, the ionosphere, and the distance to the satellite can be estimated, the uncertainties due to these effects contribute timing errors at the several nanosecond (10-9) level.

More precise frequency and time transfer has been demonstrated 3 in a test of general relativity using a spaceborne hydrogen maser that was compared with ground-based masers using a Doppler cancellation system operating at microwave frequencies. This system, which also cancelled ionospheric effects, was capable of time transfer stability at the 10-picosecond level over periods of several hours. This system appears to be capable of frequency comparison over 24-hour intervals at the $10^{-16}$ level and possibly at $10^{-18}$ over a few weeks. Here relativity effects become serious and will be difficult to calculate. The United States has conducted theoretical studies of this technique and has built and tested much of the hardware. There is no evidence of Soviet work along these lines.

The GLONASS system of 24 operational satellites will become the principal Soviet method of high-accuracy time and frequency distribution. GLONASS clocks are less stable than GPS clocks, but they are improving rapidly as new satellites appear. There are some significant life-expectancy problems with the GLONASS satellites. However, because the Soviet Union launches three GLONASS satellites at a time and can get them into position in about eight to 14 days, it can readily replace failed satellites. In contrast, up until now, GPS satellites have been launched one at a time (although there are plans to launch two at a time) and it takes about six months to make a major change in the position of a GPS satellite.

The most recent Soviet GLONASS satellites are equipped with cesium standards, and future satellites will likely have passive hydrogen masers. The existing system is capable of about a 10-meter position accuracy. Time stability data measured from the spacecraft are about 50 nanoseconds from day to day and include possible satellite positioning errors, inadequate compensation for the ionosphere, antenna multipath, and receiver noise. There is also some indication that the coordinate system used in GLONASS is different from that used in 
GPS, and that toward the poles, the differences seem to be the largest (approaching 100 meters). Soviet theoretical expectations for satellite position determination are at the 1-meter level.

Presently, GLONASS time departs by many tens of microseconds from Coordinated Universal Time (UTC), and it is less accurate than GPS time by at least a factor of 10. It appears to be offset by a relatively constant rate. In early 1990, the Soviets made major changes to bring GLONASS time closer to UTC. An error correction message in the GLONASS transmissions provides users its time offset from Moscow time, which is supposed to agree with UTC (Soviet Union) maintained at the timing center at VNIIFTRI, which in turn, is known to have several microseconds departure from UTC (but is being brought closer to UTC). The performance of the UTC time scales in the United States is within approximately 1 microsecond of UTC. Recent indications of Soviet progress in GLONASS timing can be seen in data which suggest that Soviet researchers have a GPS timing receiver and that they are locking the GLONASS timescale to that of GPS with a fixed rate of time offset (a frequency offset). The fact that the GLONASS orbital period is 11 hours rather than the 12-hour GPS period (which is a half-period of the Earth's rotation) makes it easier for Soviet researchers to deconvolve the effects of the Earth's gravitational asymmetries when they calculate GLONASS satellite orbits.

Soviet and Western researchers are cooperating to use both GLONASS and GPS receivers to achieve accurate time comparisons between the primary cesium standards at VNIIFTRI which generate UTC (Soviet Union), and the primary cesium standards in the West.

The US Department of Transportation has signed an agreement to study the joint use of GLONASS and GPS by the US Federal Aviation Administration and the Soviet air control system. Contracts are in effect in the United States for the development of receivers that can use both systems.

\section{Loran-C, Television, and High-Frequency Radio Techniques}

The Loran- $\mathrm{C}$ navigation system has been the primary timing link between the Soviet Union and the rest of the world. Its performance as a precision timing 
system is subject to daily and yearly propagation variations. Over one day, it is possible to compare the frequency of oscillators with a precision of about 1 part in $10^{12}$.

Soviet television signals are synchronized to Moscow time and, in turn, related to UTC (Soviet Union). As in the GPS/GLONASS systems, TV timing by satellite requires knowing the satellite position relative to the receiving Earth station. Despite limitations in the accuracy of the information for all users, Soviet researchers report a timing capability to all major cities in the Soviet Union with an error of less than 1 microsecond.

The stability of time transfer with television signals is at the 1-nanosecond level over line-of-sight paths. The ultimate limitation, which is due to the propagation medium, is about 100 picoseconds. Soviet receiving equipment currently has a limitation at about 20 nanoseconds. Better receivers could substantially improve TV timing in the Soviet Union.

There is a network of radio transmitters broadcasting time signals throughout the Soviet Union, which is similar to broadcasts made in the United States from Colorado and Hawaii (operated by the National Institute of Standards and Technology) and to broadcasts made in other countries. The Soviet high-frequency stations are located in Tashkent, Irkutsk, Novosibirsk, and Moscow. The accuracy of time service, allowing for delays in propagation, is about 1 millisecond. Transmission at low frequencies is done from Moscow, Irkutsk, and Novosibirsk. These frequencies provide a time accuracy within 100 microseconds over distances of several thousand kilometers. There are five very-lowfrequency (20 to $25 \mathrm{kHz}$ ) transmitter sites located at Molodechno, Arkhangel'sk, Khabarovsk, Frunze, and Gor'kiy that operate a few hours a day to transmit standard frequency and time signals. These signals are in a band capable of worldwide coverage and appear to be capable of serving as a backup for the US Omega navigation system. Soviet transmitters appear to use rubidium gas cell frequency standards.

The traditional high-frequency, low-frequency, and very-low-frequency methods of time transfer will continue to be used in the Soviet Union. The 
extent of their future coverage and hours of operation will depend on Soviet economic conditions.

\section{Meteor Trail Reflection Techniques}

The Soviet Union is unique in its use of ionization from meteor trails to reflect time signals transmitted in the very-high-frequency band over long distances. The best frequencies for this technique are in the television bands; interference apparently is not a serious problem in the Soviet Union, as the timing operations are conducted after 2 a.m., when there is little or no television activity. Historically, the Soviets have obtained between a 100- to 200-nanosecond accuracy over distances of 2,000 kilometers. Recent time closure results made over three legs of a triangle are in the 20-nanosecond range. At present, this method of point-to-point time transfer is operationally the best method for communicating highly precise time and frequency over long distances in the Soviet Union. This technique is unique to the Soviet Union and it has been in use for many years. Because this method requires a transmitter and a receiver at both ends, and operates in the same manner as the communication satellite system discussed earlier, it is not a means for general dissemination.

Because the meteor ionization trail technique has been used successfully for many years, and since there has been a substantial investment in its development, it will probably continue to be used, with some minor improvements, until GLONASS receivers replace this technique.

\section{Time Transfer by Transportable Hydrogen Maser Clocks}

Another method that is unique to the Soviet Union is the use of portable hydrogen maser clocks for transferring time. Two new maser designs have been developed specifically for this purpose, and one has been successfully used. The operational maser has a small dielectrically loaded cavity resonator made from a sapphire crystal. This maser has been used as a portable clock on trips to Cuba, where there is an ensemble of six Soviet hydrogen maser oscillators, and on trips to the Paris Observatory. 
Another type of maser that is still under development uses a spherical cavity resonator made of fused quartz and operates as an active oscillator. This maser has a servo-controlled magnetic field cancellation system that provides a 200,000fold reduction of ambient field variations during transport.

The small, portable Soviet hydrogen masers appear to be superior to their cesium beam and rubidium gas cell clocks for time transfer.

\section{Time and Frequency in Soviet Communications}

Modern communication systems with high data rates require a high degree of frequency conformity. International agreements have attempted to specify 1 part in $10^{11}$ as a goal, an accuracy which is difficult to achieve in the long term among the independently operated rubidium gas cell frequency standards that are prevalent in the Soviet Union. Western systems operate by using a hierarchy of cesium beam standards that control rubidium and quartz standards. To maintain conformity in their national systems, the Soviets have equipment that maintains the relative phase of the station oscillators, so that while their frequency differences are held to better than 1 part in 1011, the network's absolute frequency can vary far beyond this limit. This system does not meet the frequency accuracy requirements recommended in international agreements. (There is pressure from Japan to raise the international standard of frequency conformity to 1 part in $10^{13}$.)

There is also a need for time synchronization throughout communications networks to obviate the requirements of large data storage buffers at the receivers to prevent data slippages. At present, the Soviet goals appear to be far more modest than those of the United States. While there is little information available on Soviet communications plans, it is clear that a revision of Soviet frequency conformity systems will be necessary if the Soviet Union is to stay in step with the rest of the world.

\section{E. METROLOGY AND STATISTICAL REPRESENTATIONS}

Time and frequency measurement methods and the criteria by which the performance of clocks and oscillators are judged in the Soviet Union are similar 
to those in the West. Because Soviet researchers have concentrated on different types of clocks, their applications of time and frequency metrology differ somewhat, as does some of their equipment. The Soviet goal to develop a combined time-length standard is still a long way from being realized, despite significant progress.

One typical frequency measurement method at 16 significant digits involves the comparison of two signals in the gigahertz $\left(10^{9} \mathrm{~Hz}\right)$ range that are nearly alike in a frequency mixer (a device that produces a signal at the frequency difference). When the difference frequency (which is often at a few hertz, or even lower) is measured, a change of 1 hertz represents a fractional change on the order of one billionth of the frequency being measured. The trick, then, is to get the frequency to be compared as high as possible by frequency multiplication, mix it with a reference signal at nearly the same frequency, and analyze the difference. All of this must be done with due regard to possible degradation of the signals by the multipliers, the mixer, and the accuracy of the analyzer. The relative stability of a pair of oscillators is determined from the stability of the difference frequency.

Time difference measurements are done by using sharply defined time pulses to start and stop a stream of high-frequency, precisely generated pulses that are counted to give the intervals between the start and stop pulses. To make relative time readings between clocks $A$ and $B$, clock $A$ would generate the start pulse, and clock B would generate the stop pulse. The relative time stability of a pair of clocks is determined from the statistical distribution of the counted pulses. The best pulse measuring equipment in the West has an accuracy at the 100-picosecond level, and a measurement stability of about 20 picoseconds from second to second.

Sophisticated Soviet equipment has been developed for calibrating the time delay of coaxial transmission lines. The speed of the logic operations and the resolving power of this equipment is competitive with Western devices.

In performing conventional time comparison measurements between clocks at VNIIFTRI, Soviet researchers were observed to be taking measurements with equipment whose noise, for the one-hour intervals between measurements, was greater than the clock noise. Daily measurement intervals, over which the 
equipment noise is less than that of the clocks', would be more efficient and as accurate. This apparent lack of logic in the use of statistics is not typical of Soviet operation. The same approach is often used in the West, and the Soviets may have copied this inefficient approach.

During a recent visit to the United States, Soviet scientists from the Gor'kiy "Quartz" NPO displayed two hydrogen masers with a phase difference measurement system working at $100 \mathrm{MHz}$ in the active maser. These masers had 1-picosecond phase difference resolution, and could be used to measure the frequency difference between two standards with a precision of 1 second at 10-13, of 10 seconds at $10^{-14}$, of 100 seconds at $10^{-15}$, and 1,000 seconds at 10-16. These masers had visual read-out capability only. The circuit layout displayed no new developments. The Soviets stated that a microprocessor and digital hard-wire output were next in their plans. If marketed in the West at a reasonable price, these masers could be competitive.

A phase difference measurement system developed and marketed in the West has nearly comparable performance, and it works at the more convenient frequency of $5 \mathrm{MHz}$. If this system were made to operate at $100 \mathrm{MHz}$, it would be superior to the Soviet system by a factor of 20 .

Time and frequency measurements via GLONASS are dependent on knowledge of the satellite positions. GLONASS orbits do not appear to be as accurate as the GPS orbits. The 11-hour orbits of GLONASS in contrast to the 12-hour orbits of GPS should facilitate the isolation of effects of the varying gravity field of the Earth over the orbital period from other systematic measurement errors that are characteristic of the relationship between orbital periods and Earth rotation period. Soviet researchers state that the error from gravity field uncertainties in their position estimate of a satellite after 30 days ( 60 orbits) should not exceed 100 meters. They are apparently devising accurate high-speed algorithms for computing predictions. The Soviets are not yet fully appreciating their theoretical results.

Soviet researchers have conducted thorough studies on laser tracking of satellites in an effort to obtain a position accuracy of 1 centimeter. These studies appear to be more advanced than those of the West. The GPS system would ben- 
efit greatly from an independent means of position determination that is separate from the use of time-delay measurements from the onboard clock, which unavoidably includes clock errors. Information on how Soviet researchers get data to determine the GLONASS orbits is not yet available. There is one tracking station near Moscow and a second station may exist. It is possible that they have a satellite-to-satellite range-distance measuring capability on the GLONASS spacecraft, which would be a tremendous help in calculating orbits and would require only one tracking station. This "cross-link ranging" capability is planned for the GPS satellites to be launched in 1995. Soviet researchers give conflicting answers when asked if they have cross-link capability in GLONASS; our best guess is that they presently do not have this capability.

Soviet time and frequency instrumentation generally is not as advanced as that of the West, with two notable exceptions: (i) their line-length measurement device, and (ii) their optical-to-microwave frequency divider chain. Soviet laboratory techniques and equipment generally are conventional and a bit behind the West. The Soviet Union is ahead in modeling the time-delay effects of the ionosphere at very low frequencies, and the United States could take advantage of Soviet wor? in its Omega system.

The Soviet Union has expressed a desire to participate in the timing of millisecond pulsars. Some of these have extraordinarily predictable long-term rates of rotation that are comparable to the frequency stability of the best available time scale generated by atomic clocks from year to year. There are Soviet plans to build an even bigger radiotelescope than the 300-meter instrument in Arecibo, Puerto Rico, and there is an active Radioastron program to launch radiotelescopes in Earth orbit that will be free of the Earth's ionospheric and atmospheric noise.

Future Soviet progress obviously will depend on economic conditions. The extraordinary changes in the last few years in the political climate between the United States and the Soviet Union have made cooperative ventures possible that 20 years ago were unthinkable. Cooperation on space-related work, such as on GLONASS and GPS, the possible participation of the United States on the Soviet Radioastron spaceborne radiotelescope program, and the direct US inter- 
action with Soviet hydrogen maser scientists during their visit to the United States with their latest clocks, have opened a new era in timekeeping.

\section{F. RELEVANT SCIENCE APPLICATIONS IN VERY LONG BASELINE INTERFEROMETRY, RELATIVITY AND GRAVITATION, AND QUANTUM MECHANICS}

The scientific fields of experimental relativity and very long baseline interferometry (VLBI) in radioastronomy require oscillators that have the highest possible stability over the course of the observation interval (on the order of hours); both of these fields are important to timekeeping. The VLBI technique involves the recording of microwave noise signals from extremely distant objects by two or more widely separated stations. The signals, most often recorded on tape, are then brought together to be correlated. By measuring the time delay required for correlation of the data from the two stations, the angle between the baseline separating the stations and the line of sight to the noise source can be determined. Here, the oscillators at the stations play two roles: (i) that of a local oscillator used to transfer the frequency of the microwave noise to a lower frequency, capable of being recorded; and (ii) that of a clock to time tag the recorded information. This method provides the highest resolution of any form of astronomy. VLBI systems operating at $100 \mathrm{GHz}$ have resolved angles as small as 50 microarc seconds, the angular equivalent of a handprint seen from Earth on the surface of the moon.

Measured over intercontinental distances, changes of baseline length provide geological information about continental drifts. Measurements of the angle between the line of sight to a distant radio star and the baseline provide an accurate representation of Earth rotation-the original method of timekeeping.

Operation of a VLBI experiment with baseline distances of extraterrestrial dimensions has been demonstrated using the NASA tracking and data relay 
satellite system as one of the widely separated radiotelescopes. ${ }^{4}$ There are many advantages to spaceborne operation of VLBI, as there is no limit to baseline distance and no propagation restrictions on the frequencies of operation. The Radioastron program, currently underway in the Soviet Union, plans to operate a 10-meter diameter radiotelescope in a high eccentricity 24-hour orbit with terrestrial stations in several countries simultaneously collecting data. In terms of angular resolving power, the effective antenna diameter of such a system would be equivalent to the major axis distance of the spacecraft's orbit, some 84,000 kilometers.

Effects of relativity and gravitation appear whenever time is compared between clocks in relative motion or at different gravitational potentials, or combinations of these situations. Testing theories of gravitation requires oscillators with the best possible frequency stability. Conversely, precise comparison of clocks in motion or at separated locations with different gravitational potentials requires the application of corrections for relativistic effects.

Aside from general relativity, the aspect of physics most relevant to clocks and frequency standards is quantum mechanics; Soviet physicists have made strong contributions to applications and measurements related to quantum processes.

\section{Soviet Very Long Baseline Astronomy Astrophysics Programs}

Soviet radiotelescopes capable of VLBI operation are located at Simeiz on the Crimean peninsula, at Pushchino $100 \mathrm{~km}$ south of Moscow, and at Ulan-Ude in the Transbaykal. There are deep-space tracking stations suitable for VLBI located at Yevpatoriya (a former military station), and in the Far East in Ussuriysk (near Vladivostok). The exact coordinates of the last station have not been released. A major installation is under construction in Suffa, near Tashkent, which is

4 G. A. Levy, C. S. Christensen, J. F. Jordan, R. A. Preston, C. D. Edwards, R. P. Linfield, S. J. DiNardo, L. Skjerve, J. S. Ulvestad, T. Hayashi, T. Nishimura, T. Taskano, T. Yamada, T. Shiomi, H. Kunimori, N. Kawaguchi, M. Inoue, M. Morimoto, H. Hirabayashi, B. F. Burke, A. Whitney, D. L. Jauncey, C. H. Ottenhoff, K. Blaney, and W. Peters, "Results and Communications Considerations of the Very Long Baseline Interferometry Demonstration Using the Tracking and Data Relay Satellite System," Acta Astronautica, 15, 6/7(1967), 481487. 
intended for millimeter wavelength signals. Completion is planned for 1993, but with the civil unrest and severe economic problems in the southern regions of the Soviet Union, this date probably is optimistic.

Dr. N. Kardashev, the Radioastron program director, met with Dr. Vessot during his visit to Moscow in 1988 to discuss the possibility of obtaining a space hydrogen maser from the SAO for use in Radioastron. At NASA's request, Dr. Vessot and Dr. R. Decher of the NASA Marshall Space Flight Center prepared a report on the risks and benefits of such cooperation. There are preliminary indications that NASA plans to cooperate by operating Earth stations in support of the Radioastron program.

An initiative proposal by the worldwide community of roughly 70 radioastronomers recently has been sponsored by the European Space Agency called the "International VLBI Satellite." This will be a second generation of the Radioastron concept.

Both the Radioastron program and the new antenna in Tashkent are likely to suffer from the current Soviet economic crisis. There will likely be a threeyear delay in Radioastron because of the desire to operate in an orbit having greater ellipticity so as to lengthen the baseline distances and increase the coverage of southern hemisphere observations. This will require more powerful data downlink transmitters. This delay could put Radioastron at risk of being outpaced by planned Japanese and European spaceborne VLBI programs.

Soviet radioastronomers working with VLBI are competent and seem to be well supported, but their space program seems to suffer from a lack of coordination between the system engineers and scientists developing the program. The Soviets have the capability of using reliable and conventional space technology for launching scientific space payloads, but their recording and data handling equipment for VLBI operations is lagging that of the United States. As stated earlier, Soviet hydrogen maser technology is highly advanced, and in some respects, ahead of the West. 


\section{Relativity and Gravitation Studies}

Soviet researchers have been active in both the experimental and theoretical aspects of gravitation and relativity. The first ustempt at using space technology to measure the gravitational and relativistic warping of time scales was performed by Basov in 1965 with an ammonia maser in an orbiting spacecraft. The relativity results were inconclusive because of the 10-11 accuracy of clock comparison, but the basic technique for cancelling the first-order Doppler effect in the communication system proved to be sound, as did the theoretical work done to support the test. Eleven years later, in 1976, a test was performed in the United States by Vessot and Levine of the Smithsonian Astrophysical Observatory (SAO) using a rocket probe going to the much higher altitude of 10,000 kilometers carrying an atomic hydrogen maser. This test confirmed the predictions of general relativity at the $70 \times 10^{-6}$ level.3,5

A new and fundamentally different approach to a theory of relativistic gravity was taken in 1985-1986 by Yu. M. Loskutov, a prominent Soviet academician. The soundness of this theory was questioned by expert Soviet theorists despite the, then, considerable political risk. The new theory has had little success anywhere, but generally, the Soviet contributions to this field have been solid and highly regarded.

Soviet theorists, with their enviable mathematical prowess, are highly competent, and will continue to make valuable contributions in the field of relativistic gravitation. Their application of theory to the GLONASS program is solid and incontestable. Experimental opportunities with spaceborne clocks in the forthcoming Radioastron program could advance the precision of the 1976 SAO test from $70 \times 10^{-6}$ to $1 \times 10^{-6}$.

\section{Quantum Metrology}

There are fundamental limits imposed on the stability of oscillators by quantum theory. At temperatures where the thermal energy-per-unit bandwidth

5 R. F. C. Vessot and M. W. Levine, "A Test of the Equivalence Principle Using a Spaceborne Clock," General Relativity and Gravitation, 10, 3(1980), 181-284. 
exceeds the quantum mechanical zero-point energy in an oscillator, thermal noise will set the limit of its frequency stability. However, by increasing the resonance $Q$ of an oscillating system operating at very low temperatures, conditions can exist in macroscopic devices where the quantum effects will dominate. This can apply to both mechanical and electromagnetic resonators. While present oscillators have, by far, not reached quantum limits, the limits seem approachable, and are the subject of serious study in both the Soviet Union and West.

Another area where quantum processes play a role in precision measurement is the direct result of the uncertainty principle. Certain pairs of such quantities as position and momentum, energy and time, and others, are said to be canonically conjugate, and the product of their uncertainties has a finite lower bound. The same type of thinking has been applied to the frequency of the photons of electromagnetic signals in a waveguide. New quantum states for photons have been discovered and their properties are being investigated. The Soviet Union is leading the West in these theoretical studies, and there is increasing probability that successful experiments will follow.

Experimental high $Q$ resonator apparatus consisting of aluminum bars weighing several tons enclosed in vacuum systems and cooled to extremely low temperatures are used in attempts to detect gravitational radiation. This detection is the "Holy Grail" of modern experimental physics, and is being pursued by many nations, including the Soviet Union. Pioneering studies on how to cope with the quantum limitations to detecting the motion of these systems have been conducted by V. B. Braginskiy of the Moscow State University. Cooperative studies are now underway by top theorists in both the Soviet Union and the United States.

There is likely to be increased collaboration between Soviet and US scientists in this fundamental work, which grows in importance as experimental work moves closer to fundamental limits. Important applications in communications and in areas of metrology are foreseen for this theoretical work. Professor V. B. Braginskiy worked with Professor Kip Thorne at the California Institute of Technology for approximately three months during the winter of 1990-1991; this is the continuation of a long and productive relationship. 


\section{G. AVAILABLE SUPPORTING TECHNOLOGY}

In addition to the normal complement of electronic systems and components, atomic frequency standards require electronics that fall in several unique categories such as quartz-crystal-controlled oscillators, frequency multipliers, frequency synthesizers, and very-low-noise electronics. While few or perhaps none of these require new or exotic components or technology, there is a strong requirement for reliable operation over long periods of time, and this requirement generally is met by conservative design.

\section{Quartz Crystal Resonators}

Quartz-crystal-controlled oscillators are the backbone of atomic clocks inasmuch as they are controlled in various ways by atomic resonances and are used to provide the actual signal that is delivered to the user. Quartz resonators operate by vibrating mechanically with very high $Q$, that is, with very little mechanical damping. Quartz crystals also have piezoelectric properties that allow the transducing of electric fields to mechanical motion. When properly equipped with electrodes, the quartz behaves as an electromagnetic resonator with quality factors, or $Q$ values, as high as $10^{7}$.

Being a mechanical resonator, the frequency of a quartz crystal is sensitive to vibration, acceleration, and temperature changes. A number of techniques have been discovered to minimize these environmental effects. The most significant of these involve: (i) the method of supporting the quartz wafer; (ii) the "cut" of the crystal (in the sense of the cut of a precious stone); that is, the size of the wafer, the mode of resonance, and the orientation of the crystalline axes to the faces of the oscillating wafer; and (iii) the method of controlling the temperature of the wafer or of compensating for the temperature-induced resonance frequency variation. The quality of the natural or synthetic quartz is also important. The Soviet Union has abundant quantities of high-quality natural quartz.

Soviet quartz oscillators do not appear to be produced using the most vibration-immune and temperature-insensitive "doubly rotated AT cut," although the technology appears to be well understood. They use the conventional "AT" cut that has good temperature insensitivity and employ temperature-control 
techniques. Recently declassified US technology that is based on computer reconstruction of a temperacure-corrected signal from a non-temperature-controlled oscillator in order to avoid large dimensions and power consumption of thermal controllers was reported in the Soviet Union long ago. This technique involves simultaneously operating a quartz oscillator in both a relatively temperatureinsensitive mode and a temperature-sensitive mode. By measuring the frequency difference, the quartz temperature can be computed, and the appropriate frequency offset can be synthesized to bring the oscillator output to its calibrated frequency.

\section{Frequency Multipliers and Synthesizers}

The Soviet development of frecuency multipliers has followed the usual technology available worldwide. It is only in the remarkably energetic Soviet laboratory efforts to realize a phase coherent link from microwave-to-optical frequencies that progress is comparable or perhaps slightly ahead of the West.

In digital frequency synthesizers, the Soviet system appears to have reaped a bonanza of patents, principally in the area of "number-controlled" oscillators that are used for rapid, phase-continuous changes of frequency. These appear to be conventional devices that are competently designed and appropriately used in Soviet equipment.

\section{H. SOVIET INSTITUTIONS INVOLVED IN TIME AND FREQUENCY TECHNOLOGY}

The strong and prestigious scientific research institutes involved in time and frequency technology that are affiliated with the Soviet Academy of Sciences include the Lebedev Physics Institute in Moscow, the Thermal Physics Institute in Novosibirsk, and the Spectroscopy Institute in Troitsk. In addition to these scientific centers, there are numerous universities and technical institutes which train engineers at various levels of graduate degrees. There is an impressive number (more than in the United States) of applied technology educational institutes involved in precision timekeeping which educate technologists and contribute to improved timekeeping. 
The Soviet university structure appears to be more similar to that of late 19th century Europe than to the present structure in the United States, in that there is a much more formal attitude toward authority in the operation of a laboratorytechnicians do technical work, engineers do engineering, and machinists cut metal. The typical young North American graduate student's experimentalist attitude toward hands-on experimentation, which includes performing his own machining and wiring (bred from working on a jalopy of some kind, or tinkering in a basement full of tools), simply does not exist in the Soviet Union. This type of individual is rarely seen in Soviet culture; however, this type of individual is a strong player in the US capability for generating new ideas and techniques and realizing practical equipment from original ideas. This is the essential capability of closing the gap between theory and applied technology for which the United States is justly notable.

There are considerably more professionals involved in precision timekeeping in the Soviet Union than in the United States, with an impressive capacity to deploy, operate, and maintain timekeeping equipment.

The Soviet industry (in the US sense) that performs product development and engineering for quantity production, manufacturing, and testing of commercial and military timekeeping equipment is inferior to that of the United States. The infrastructure to support this activity by providing special materials (metal alloys, heat treatment, magnetic materials) and advanced electronic components, and the availability of specialized vendors to perform quartz glassblowing, metalspinning, electroplating, and optical grinding and polishing is not nearly as advanced or accessible as that in the United States.

It is likely that future major advances will continue to be made in the West. It is also likely that the Soviet Union will continue to adopt Western advances. In view of the Soviet high level of effort, improved access to foreign materials and subassemblies, and the quality of qualified practitioners, a high degree of Soviet innovation can be expected.

The industrial capability of the Gor'kiy R\&D Instrument Making Institute is somewhat enigmatic at this time. The hydrogen maser products described are impressive, and it will be interesting to see how reliable they will be. The ability 
of the Soviet Union to enter into foreign markets will depend on how well it can refine its designs for economical (even profitable) manufacture, distribution, and maintenance in a foreign environment. At the time this report was prepared, a team of three Soviet hydrogen maser scientists from the Gor'kiy "Quartz" NPO had visited the Smithsonian Astrophysical Observatory (24 September 1990 through 15 October 1990). They brought along two hydrogen masers (their models Ch1-75 and Ch1-76) for a series of performance comparisons with hydrogen masers built at the SAO and with hydrogen masers at the US Naval Observatory. The outcome of the comparisons was reported at the 1990 Precise Time and Time Interval Meeting held in McLean, Virginia. Excerpts from this paper are included in Appendix A. 


\section{CHAPTER II \\ INTRODUCTION AND TUTORIAL}

\section{A. SUMMARY}

Time and its reciprocal, frequency, are the most accurately and precisely realized of any physical parameter. At present, 16 significant digits are often required to represent data, and modern metrology is strongly based on measurements involving accurate frequency. The words "clock" and "frequency standard" are often used interchangeably in most literature, and in most contexts the terms refer to a highly stable oscillator. It should be remembered that a clock can provide dating of events on a time scale, while a frequency standard provides the elementary interval or unit of time.

\section{B. THE METROLOGY OF FREQUENCY AND TIME: A REVIEW OF PRESENT DEFINITIONS OF DISTANCE AND TIME}

Since 1967, the unit of time has been defined as the atomic second, derived in terms of the quantum mechanical interaction between the magnetic dipole moment of the nucleus and that of the outer (or valence) electron of the cesium 133 atom in its lowest electronic energy state at zero magnetic field and at rest with respect to the observer. This interaction, known as ground state hyperfine energy separation, provides a natural resonant frequency, which in cesium 133 is defined as $9,192,631,770 \mathrm{~Hz}$. This is the present basis of the System International (SI) second of time. While cesium 133 is chosen for the current definition, several types of quantum mechanical processes involving other atoms, ions, and even molecules are useful in producing highly stable oscillators whose frequency can be related to the defined frequency.

In 1983, the velocity of light was taken as a defined quantity in the SI system of units. The definition of the meter is now taken as the distance light travels in free space in $1 / 299,792,458$ of a second. Today, time measures distance; the cesium 133 atom now serves both as a clock and a yardstick.

An indication of the progress in clock development since 1000 A.D. is shown in Figure II.1, which shows the day-to-day stability of the best clocks at the dates 
indicated. Currently, time stability appears to be improving by roughly a factor of 10 per decade.

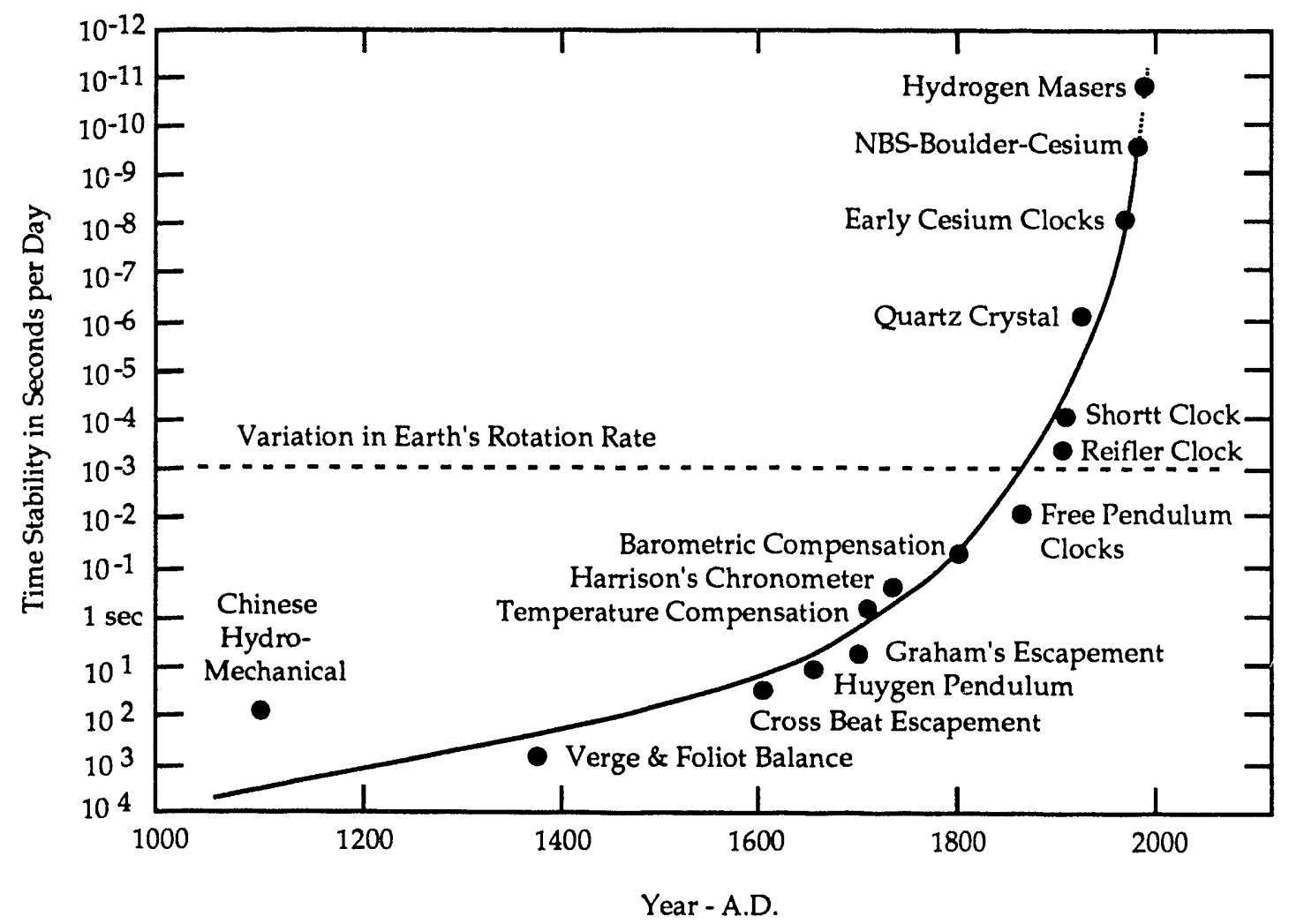

Figure II.1

Progress in Clock Development

It is important to recognize both the difference in the concepts of time and time interval, and the distinction between time and frequency accuracy and time and frequency stability. Our intuitive concept of time is the accumulation of regular intervals-years, months, days, and hours-and the most common measures are done with the calendar or with a count of days in a cumulative numerical sequence, such as the modified Julian date. On the other hand, time interval is now defined in terms of the atomic second, a process that depends on quantum mechanical interactions in the cesium 133 atom. 
Time accuracy refers to the agreement of a clock with respect to a defined time scale such as Universal Coordinated Time. This concept becomes important in network synchronization.

Because the rotation of the Earth is irregular, the number of atomic time seconds is different from year to year. The present convention is to adjust the number of atomic-time seconds as needed with a leap-second so that the defined atomic time is never more than 0.9 seconds different from the time defined by the Earth's rotation. Sixteen leap seconds have been added between 1972, when the leap-second was introduced, and 1990. This process is totally analogous to the numerical problem posed by the length of the year and the number of Earth rotations that was resolved in 1582 by edict of Pope Gregory XIII with the invention of the leap year.

\section{FREQUENCY ACCURACY AND FREQUENCY STABILITY: TIME ACCURACY AND TIME STABILITY}

The concepts of frequency accuracy and frequency stability are quite different. Frequency accuracy is the extent to which the physical manifestation of an oscillator can be independently realized in terms of the defined unperturbed hyperfine resonance frequency of cesium 133, taken at rest and at zero magnetic field. In the cesium frequency standards of the various national standards laboratories, care is taken to account for all possible departures from these idealized conditions. Currently, the accuracy capability claimed by the best standards is on the order of 1 part in 1014, when all known effects are accounted for.

The concept of frequency stability requires the understanding of the spectral distributions of the frequency fluctuations or alternatively, of the phase fluctuations. For very long time intervals, the spectral distributions of phase or frequency variations must be described by extremely low Fourier frequency components (microHertz and lower). This process is cumbersome and not intuitively satisfying. More importantly, there is the problem of how to represent in the time domain the effects of spectral distributions that cause the variance to diverge. This problem was solved by Allan in 1966 by taking a finite number, $\mathrm{N}$, of samples for the representation of the variance; the value $\mathrm{N}=2$ is very convenient and has been universally adopted. The description of frequency stability 
that is now adopted is the Allan "variance" (or its square root, the Allan standard deviation), $\sigma_{y}(\tau)$, where $y$ signifies fractional frequency, $d f / f$, and $\tau$ signifies time interval. Simply put, the Allan standard deviation (sometimes called the twosample deviation) is the one-sigma probability of the fractional frequency difference between time-adjacent measurements, each of duration $\tau$ seconds. Figure II.2 illustrates the process by which the Allan standard deviation is defined. Of course, there are no ideal clocks with which to compare. In some cases, the reference clock is much more stable than the clock under test, and it does not matter. If two identical clocks are compared, the Allan standard deviation is assumed to be equal for both and is $2^{-1 / 2}$ the measured value. If more than two clocks can be simultaneously intercompared, then it is possible to obtain an estimate of the performance of each individual clock, given enough statistical averaging.

The behavior of $\sigma_{\mathrm{y}}$ on $\tau$ depends on the spectrum of the frequency fluctuations and one can relate the slope of the Allan standard deviation data plotted against averaging time interval to the spectral distribution. Typically, the Allan standard deviation of an oscillator will improve as averaging time increases until low frequency processes that have a $1 / \mathrm{f}$ or $1 / \mathrm{f}^{2}$ distribution of frequency fluctuation dominate, and the variance will then deteriorate for longer averaging intervals. Such low-frequency processes are often the result of a combination of systematic effects. These systematic effects can be caused by vibration, room temperature variations, magnetic field fluctuations, seasonal changes in humidity, and a number of vaguely defined processes such as "aging." While these systematic processes generally can not be comfortably described in terms of a spectrum, nonetheless, their effects are usefully revealed in terms of the Allan standard deviation. 

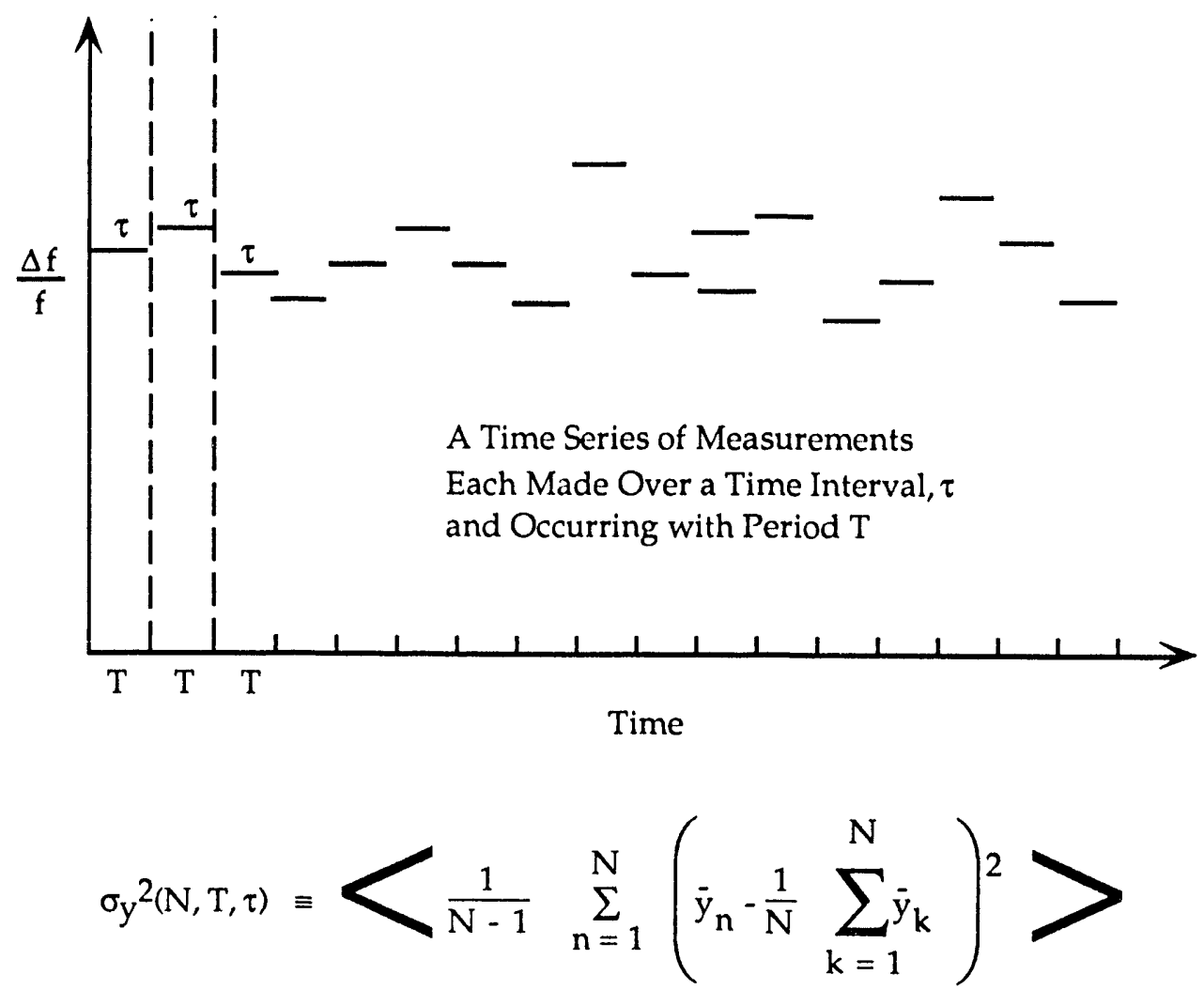

where $<>$ is the infinite time average.

$$
\begin{gathered}
\qquad \text { For } N=2 \text { and } T=\tau \\
\sigma_{y}^{2}(\tau) \equiv\left\langle\sigma_{y}{ }^{2}(2, \tau, \tau)\right\rangle=\left\langle\left(\bar{y}_{k+1}-\bar{y}_{k}\right)^{2}\right\rangle / 2 \\
\text { where } y \text { is the fractional average, } \frac{\bar{\Delta} f}{f} \text { over an interval, } \tau \text {. }
\end{gathered}
$$

Figure II.2

The Allan Standard Deviation

\section{APPLICATIONS OF FREQUENCY STANDARDS AND THE USE OF THE ALLAN STANDARD DEVIATION}

In this report, all descriptions of oscillator stability will be made in terms of the Allan standard deviation. This quantity can be used to predict the limitations imposed by oscillator stability or more properly, instability, in the measurement of range distance and range rate using microwave or laser signals. 
Probably the most exacting and demanding uses of high stability oscillators are in the microwave systems used in deep-space navigation and tracking, and in the very long baseline interferometry systems of radioastronomy.

Range distance measurements in spacecraft tracking systems use modulation codes to time-tag transmitted signals. The received signal is compared and timecorrelated with a similar modulation sequence generated at the receiver. The time interval between the two sequences gives the range distance.

Distance $=\mathrm{c}$ times (time interval), where $\mathrm{c}$ is the velocity of light.

Range rate information usually is obtained from the Doppler frequency difference, $f_{d}$, between the transmitted and received signals. For example, consider a one-way transmission at frequency, $f$, from an Earth station to a space station whose range distance is changing at a rate $\mathrm{dr} / \mathrm{dt}$. The Doppler shift in the received frequency is given by $f_{d}=(f / c)(d r / d t)$. If the transmitted signal is reflected or retransmitted (transponded) back to Earth, the measured Doppler shift is twice as large. The accuracy in measuring range-rate depends on the stability of the transmitting and receiving stations' clocks during the measuring time. A clock's contribution to the inaccuracy of the range rate, in terms of the Allan variance, can be expressed as follows:

$$
\sigma_{\mathrm{dr} / \mathrm{dt}}(\tau)=\mathrm{c} \sigma_{\mathrm{y}}(\tau)
$$

Given that $\sigma_{\mathrm{y}}(1,000$ seconds $)=1 \times 10^{-15}$, we have that $\sigma_{\mathrm{dr}} / \mathrm{dt}(1,000$ seconds $)=$ $3 \times 10^{-7}$ meters/second, or $0.3 \mathrm{microns} / \mathrm{second}$.

An estimate of time stability from the Allan variance can be obtained by writing $\sigma_{\Delta \tau}(\tau)=\tau \sigma_{y}(\tau)$. Here $\sigma_{\Delta \tau}(\tau)$ is the one-sigma expectation value of the time difference in adjacent time intervals, $\tau$. This concept can be used to determine the limits imposed by a clock (or oscillator) performance on range distance measurements. Figure II.3 shows how the time dispersion (and therefore the range dispersion limits, $\left.\sigma_{\Delta R}[\tau]\right)$ imposed by clock stability is related to the Allan standard deviation of modern hydrogen maser oscillators. 


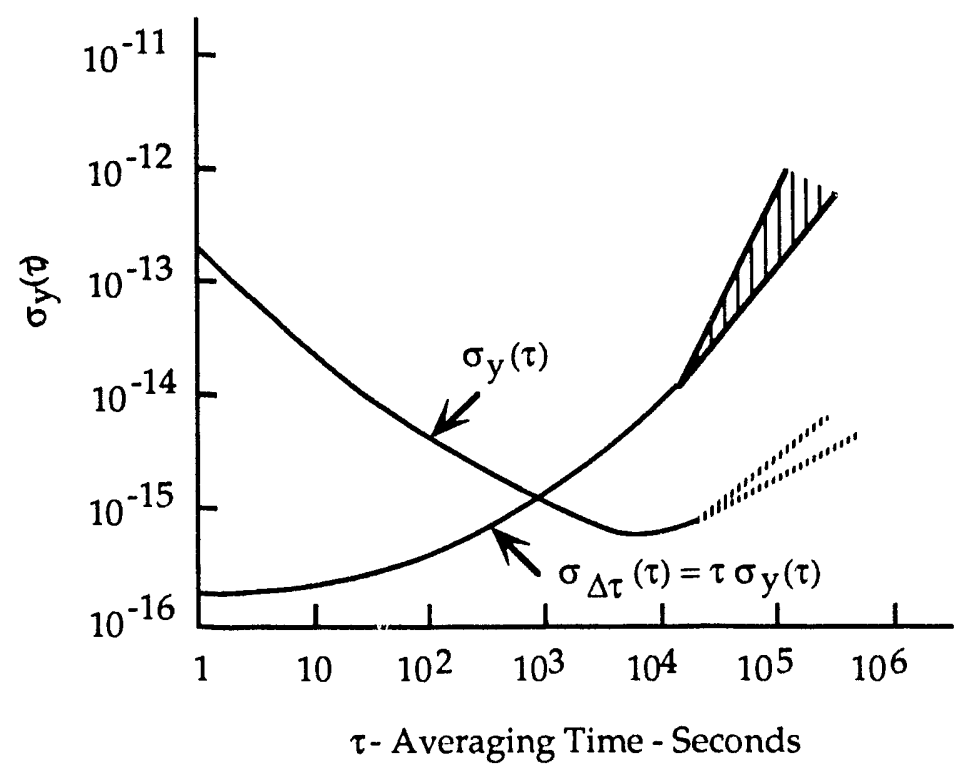

$$
\sigma_{\Delta \tau}(\tau) \quad \frac{\sigma_{\Delta R}(\tau)}{=c \sigma_{\Delta \tau}(\tau)}
$$
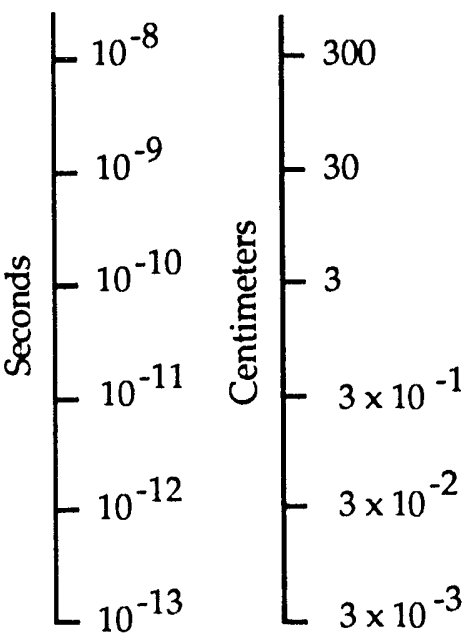

Figure II.3

Time and Range Distance Deviation from the Allan Variance for Hydrogen Masers

Measurements of angle (or of relative bearing) of a single source can be made by timing the arrivals of signals from the source arriving at two or more separated locations. This is how multistatic radars, very long baseline interferometers, and deep-space tracking systems operate. A schematic of a very long baseline interferometry system is shown in Figure II.4. The accuracy in the determination of the angle, $\theta$, between the line of sight to the signal source and the line connecting a pair of tracking stations separated a distance, $\mathrm{L}$, is given by $\sigma_{\Delta \theta}(\tau)=c \tau \sigma_{y}(\tau) / L \operatorname{Sin} \theta$

In high-precision systems that depend on the propagation of electromagnetic signals, variations in the velocity of propagation through the medium can present problems. Figure II.5 shows the typical degradation of the frequency stability of microwave signals that occurs in a single vertical pass through the Earth's troposphere and ionosphere. Here the effect of the troposphere alone is quite independent of frequency. The effect of the ionosphere, however, is complicated. To first order, the change in velocity of propagation (compared to that in 
vacuum) depends on the reciprocal of the (carrier frequency) 2 and is proportional to the density of electrons in the path. The plot marked "iono + tropo" in Figure II. 5 is for $2.2 \mathrm{GHz}$. The data for "tropo" alone were taken at $44 \mathrm{GHz}$, and do not include ionospheric effects. Figure II.5 also shows the stability of several types of frequency standards and the millisecond pulsar, to illustrate the necessity of having some method of compensating for, or accounting for, tropospheric and atmospheric propagation effects if high-precision comparisons are to be made between widely separated high-stability clocks.

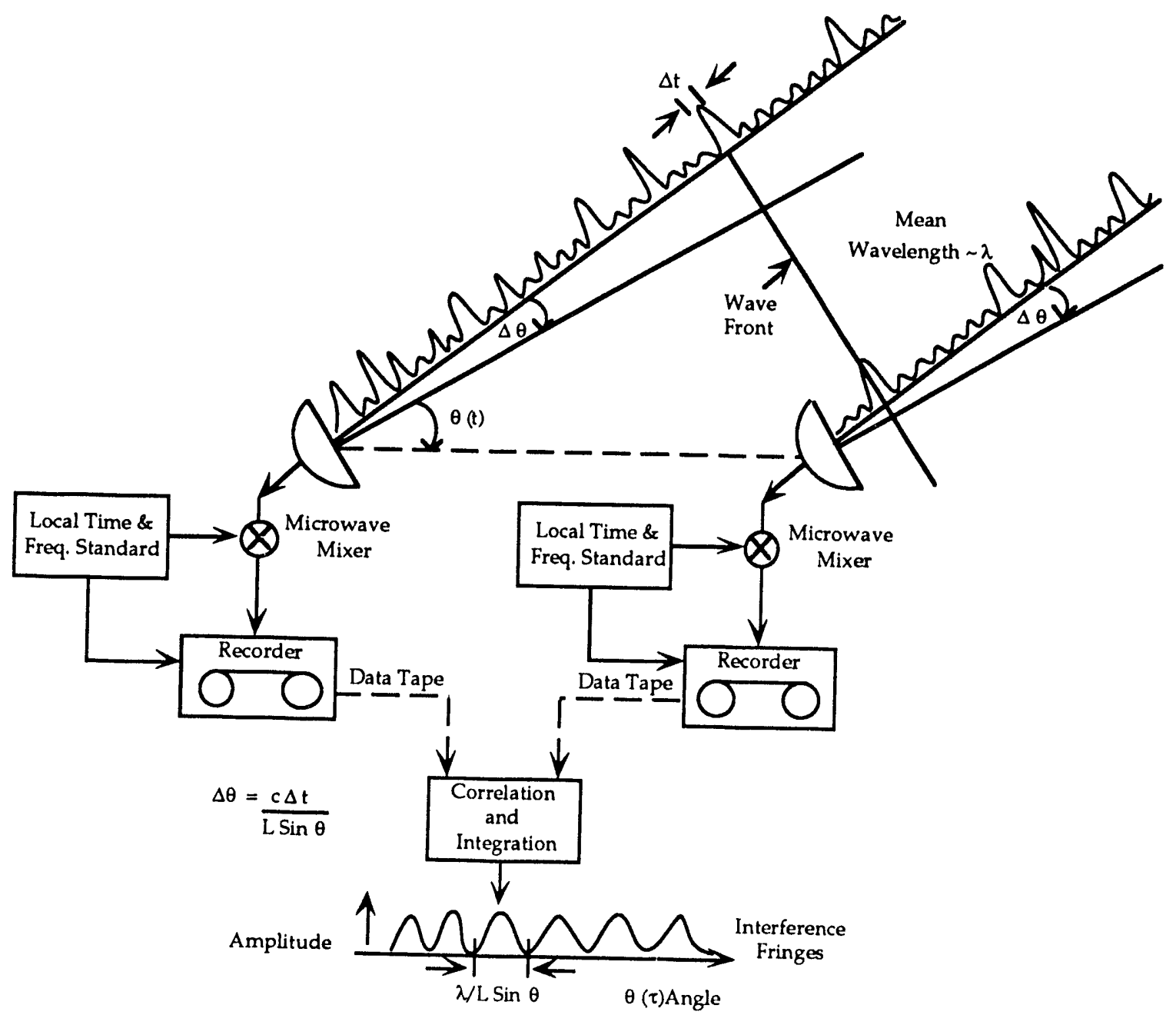

Figure II.4

Schematic of a Very Long Baseline Interferometry System 


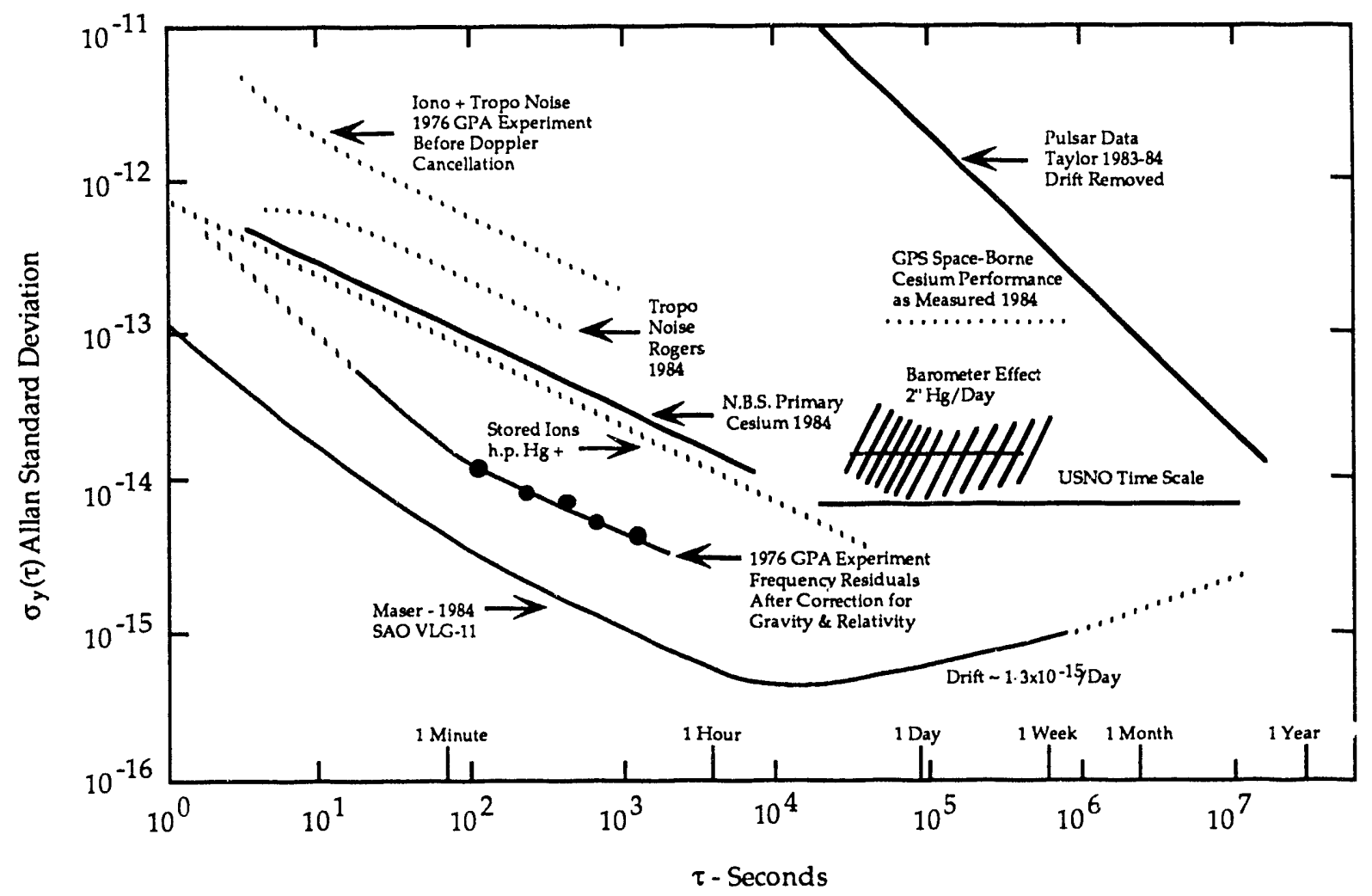

Figure II.5

Effects of Troposphere and Ionosphere on Frequency Stability

\section{E. EFFECTS OF GRAVITATION AND RELATIVITY}

The concept that time is an absolute quantity (which was one of the cornerstones of Isaac Newton's Principia published in 1685) was completely revised by the advent of Einstein's Special Theory of Relativity, and later in 1916, by his General Theory of Relativity. Thus far, this theory has met every experimental challenge within the accuracy of the devices or systems used in its test. It established the principle that the passage of time depends on the nature of the difference in gravitational potential and velocity between the observer and the clock.

Of particular interest to the issue of frequency and time intervai is the fact that we must account for gravitational and relativistic processes when we compare these quantities at very high precision. If we crmpare clocks that are in relative motion, we must account for time dilation; each clock that is in motion rela- 
tive to a clock to which it is being compared will appear to run slow by an amount $\Delta f / f=v_{r e l} / 2 c^{2}$, where $\Delta f / f$ is the fractional frequency shift, and $v_{r e l}$ is the relative velocity of the two clocks.

Furthermore, if clocks are compared at different gravitational potentials, a shift of $\Delta f / f=\Delta \Phi / c^{2}$ occurs, where $\Delta f / f$ is the fractional frequency shift, and $\Delta \Phi$ is the gravitational potential difference between the clocks. Between the surface of the Earth and a point far away in space, an Earth clock will appear to run slow by about 7 parts in $10^{10}$ with respect to the space clock. If the density of a massive body is sufficiently great, the clock near this body will appear to stop when measured by the clock in space. This, of course, is the situation known to astrophysicists as the "black hole."

One of the great successes of Einstein's theory is the recognition of the constancy of the velocity of light. In 1983, the velocity of light was taken as a defined quantity in the SI system, which in turn defines $\varepsilon_{0}$, the permittivity of free space (second/ohm-meter) through the relation $c=1 /\left(\varepsilon_{0} \mu_{0}\right)^{1 / 2}$. In SI units, $\mu_{0}$ is taken as $4 \pi \times \mathrm{i}^{-7}$ (ohm-second/meter). The value chosen for the velocity of light is $2.999,792,458 \times 10^{8}$ meters/second.

Now that time interval defines distance, atoms now serve both as the clock and the yardstick in the traditional "gedanken" or thought experiments that discuss the effects of gravitation and relativity, and in real experiments to test the general theory of relativity.

\section{F. ATOMIC CLOCKS}

The fundamental process by which atomic clocks operate involves the technique of applying microwave (or light) signals to make resonant transitions between quantum states in atoms or ions. As an example, Figure II.6 shows the hyperfine structure of the ground state of rubidium 87 ( $F$ is the total angular momentum), with Zeeman splitting shown ( $\mathrm{m}_{\mathrm{F}}$ is the quantized projection of $\mathrm{F}$ along a magnetic field). A transition between the two allowed values of $F$ (that is, reversing the spin of the valence electron) releases or absorbs an energy difference $\Delta W$ that corresponds to a quantum of radiation in the gigahertz range. These transitions are detected by a number of different techniques, including the 
detection of a coherently amplified applied signal, or of an oscillation powered by transitions, as in masers and lasers. The most important feature of atomic clocks is that these transitions can be made to occur without significant interference from perturbations other than the applied signal. A high degree of isolation of the atoms or ions is essential. The longer the atom or ion is accessible without excessive perturbation, the narrower is the range of frequencies to which the resonance transition will respond. The relationship between the width of the resonance, $\Delta f$, and the period of time of unperturbed access to the atom, $\Delta t_{o b s}$, is given by the Heisenberg uncertainty relationship, $\Delta f=1 / \Delta t_{\text {obs }}$. The effective $Q$, or quality factor, is defined by $Q=f / \Delta f$, where $f$ is the frequency of the resonant interaction. It is clear that the higher the frequency, and the longer we can extend $\Delta t_{\text {obs, }}$, the better the $Q$.

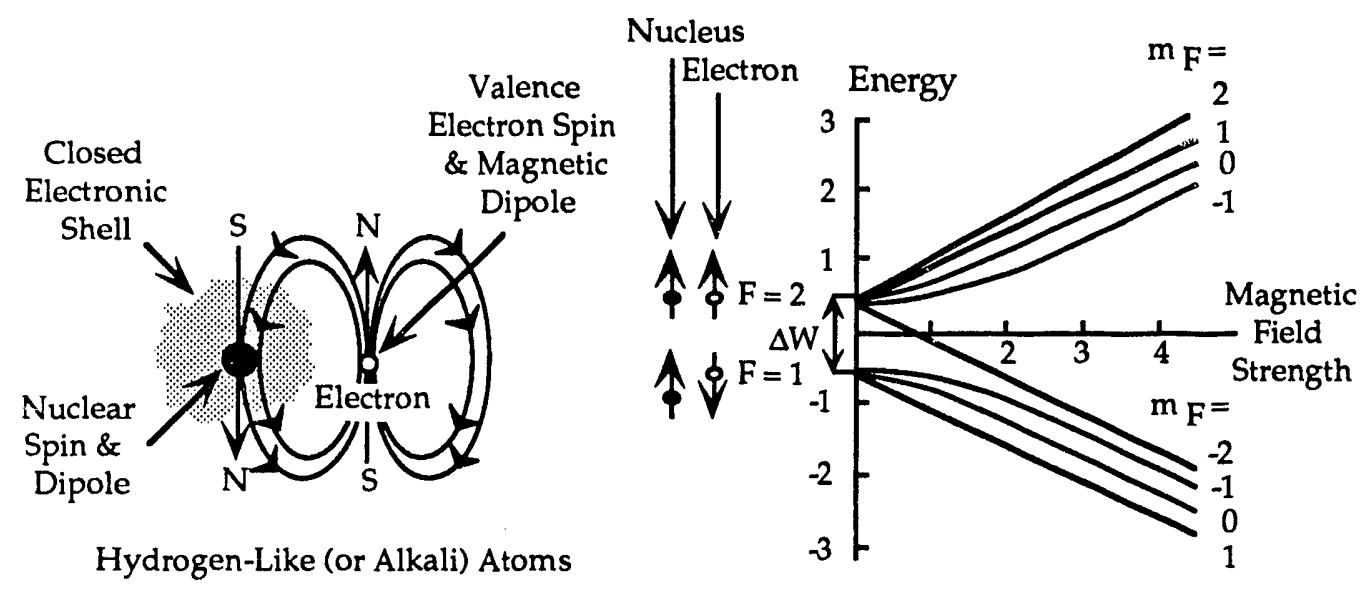

Figure II.6

Hyperfine Structure of Rubidium 87 with Zeeman Splitting (Nuclear Spin $[I]=3 / 2, F_{0}=\Delta W / h=6,834,682,605 \mathrm{~Hz}$ )

Another important requirement to realize a practical frequency standard is to have a good signal-to-noise level in the detection of the transitions. If we have a finite number of atoms (or ions) involved in making transitions during a time period $t_{o b s}$, making this period longer means a slower transition rate and thus a lower signal power, therefore, we must integrate (or average) for longer intervals 
to overcome noise in order to achieve a given level of stability. This means that the stability can only be realized over longer time intervals.

In evaluating the potential frequency stability of an oscillator in a statistical sense, we can define a figure of merit as $F O M=Q \cdot$ (signai-to-noise ratio).

If the noise processes are random, and have normal frequency distribution, and the frequency stability of an oscillator improves as the (averaging time)-1/2, the relationship of signal to noise and linewidth characterized by this behavior is the underlying limit to the statistical performance of all frequency standards. Though useful for establishing limits, this relationship completely ignores systematic perturbations from external events such as variations in temperature and magnetic fields and the effects of mechanical accelerations; these various effects impose the practical limitations to long-term frequency stability.

\section{G. ATOMIC RESONATORS FOR ATOMIC CLOCKS}

Atomic resonances are employed either passively or actively. In active devices such as masers and lasers, the atoms release coherent electromagnetic power when they make transitions, and this signal is converted to useful frequencies such as 1,10, and $100 \mathrm{MHz}$. In passive devices, the atoms act as a highly selective frequency discriminator or filter. The information about whether or not the quantum transitions have occurred (and to what extent) is conveyed by various detection processes and is used to control the frequency of an oscillator whose stability is compatible with the rate at which information is received from the detector.

In atomic beam resonators (or frequency discriminators), control information can be obtained from the change in trajectory of a beam of atoms passing through an inhomogeneous magnetic field that occurs as a result of the change in their magnetic dipole moments. In the case of stored atoms or ions, the transitions are detected by the absorption or emission of optical signals.

High-quality, quartz-crystal-controlled oscillators normally are used as flywheel oscillators in both active and passive atomic clocks. In passive resonators, the signal that causes the transitions is frequency modulated across the peak of 
the resonance, and the response is synchronously detected to control the frequency of the quartz oscillator.

There are many techniques for obtaining a narrow resonance with relatively low levels of unwanted perturbation. The first atomic clocks operated with wellcollimated beams of cesium 133 atoms from an oven that were deflected by powerful magnetic gradients to determine their quantum states. In this technique, the time of unperturbed interaction depends on the speed of the atoms and the distance they can be made to travel in the interaction region where they undergo quantum transitions. Cesium 133 is easily detected by ionization when it strikes a hot surface, it has a quantum transition which at low magnetic fields depends only on the square of the magnetic field, and it has a relatively high resonance frequency of $9 \mathrm{GHz}$. Because of the success of the primary cesium standards developed in various national laboratories and industries, cesium has been chosen for the primary standard. Beams of other alkali-like atoms (such as hydrogen, calcium, and thallium 205) have also been used in experimental frequency standards.

Alkali atoms such as rubidium, cesium, and potassium also can be maintained in relatively good isolation by placing them in light-transparent cells containing "buffer gases" that reduce the rate of their interatomic collisions and the resulting disturbance of their hyperfine interactions. Such gas cell devices chiefly use rubidium 87 atoms (6.8-GHz resonance) and are noted for their small size, ruggedness, and low cost in comparison to other atomic clocks. There has been considerable work on gas cell devices in the Soviet Union, and commercial devices have been offered for sale.

Atomic hydrogen maser frequency standards are active oscillators that operate by dissociating molecular hydrogen and collimating it into a beam that is led down the axis of a multipole magnet (usually 4 or 6 poles) where atoms in the two upper energy hyperfine states are focused radially inward into an interaction region. (Atoms in the two lower states diverge in the magnet and are pumped away.) The interaction region consists of a cavity resonator that contains a storage vessel. This vessel or "bulb" is coated with a material (usually teflon) which repels the atoms during their collision with the surface producing very little perturbation in the atom's hyperfine interaction. The cavity resonator is tuned to 
the frequency of the hydrogen hyperfine transition and serves both to apply the electromagnetic field to stimulate the atomic transitions and to collect the emitted energy, acting in a regenerative manner to provide continuous oscillation. The stability of the maser depends chiefly on the length of time the hydrogen atoms can be kept in the storage vessel. The magnetic isolation of the transition region and the resonance frequency stability of the cavity are crucial to the successful design of hydrogen masers.

In the United States, atomic hydrogen masers have successfully operated at temperatures near $0.5^{\circ} \mathrm{K}$. While such experiments have not been reported in the Soviet Union, a large number (hundreds) of conventional hydrogen masers are used as working frequency standards in Soviet regional timing centers. Hydrogen masers are the most commonly used high-stability frequency standards in the Soviet Union.

Extremely narrow resonances have been obtained by trapping ions using magnetic and radio frequency techniques. It is possible to isolate groups of ions, and even individual ions for periods of many days under conditions that have little effect on their hyperfine structure. Furthermore, by various collisional and electromagnetic methods, the physical movement of ions can be reduced so that their kinetic energy is equivalent to temperatures in the milliKelvin domain. These trapping techniques show great promise for standards of extraordinary stability and accuracy. There is considerable activity on these devices in laboratories throughout the world, including the Soviet Union.

The development of tuneable lasers has made possible the retardation of atomic beams and the suspension of free atoms in space by the transfer of linear momentum through optical resonance transitions. Extremely low equivalent temperatures and very long storage times have been achieved using these techniques. Soviet scientists have been active in this work.

Cryogenically cooled electromagnetic resonators with superconductor coatings offer possibilities for obtaining extremely good stability over short time intervals (seconds). The high levels of spectral purity from such oscillators is important for future flywheel oscillators to replace quartz oscillators that have insufficient stability to operate devices with extremeiy narrow resonances. Cryo- 
genically cooled high $\mathrm{Q}$ resonators made of very low loss materials, such as sapphire, can be operated without conductive surface coatings by employing internal reflection of electromagnetic fields. The Soviets have conducted pioneering research on such cryogenically cooled resonators.

Optical resonances in atoms and ions can be combined with microwave transitions to provide a combined length and time standard. The possibility of stabilizing lasers with various atomic resonances is being explored vigorously in the Soviet Union.

\section{H. CURRENT STATUS OF ATOMIC CLOCKS}

During the past three decades, the development and production of atomic clocks in the United States has followed many different directions in the wake of several government and privately supported programs. Cesium beam, rubidium gas cell, and hydrogen maser standards have been developed for both space and terrestrial use. Commercial versions of these devices have been available in the United States for decades and they have been widely exported. Some of these clocks are in use in the Soviet Union, and it is clear that the technology of these devices is well understood there. New types of clocks involving trapped ions are being developed in the United States and are in the prototype phase. There is little evidence of Soviet work on atomic clock development using trapped ions.

The frequency stability limits of several high-stability oscillators currently used in the United States are shown in Figure II.7, along with specifications for the US Global Positioning System clocks and commercially available cesium beam and rubidium gas cell clocks. The present estimated stability of the International Atomic Time Scale is also shown. The stability of the millisecond pulsar (PSR1937 + 21), with its $9.07 \times 10^{-15}$ per day linear drift removed, is also included as it, and other such pulsars which eventually will be discovered, promise to be extraordinarily reliable clocks for very long periods of time.

Figure II.7 provides a perspective for relating the available data on the performance of US clocks to those of the Soviet Union. It does not, however, include data on recent experimental devices such as stabilized lasers, trapped and cooled ion standards, cryogenically cooled hydrogen maser oscillators, or cryo- 
genically cooled cavity stabilized oscillators. Progress and developments in more recent US and Soviet devices require far more detailed attention and are reported in more detail in the following sections.

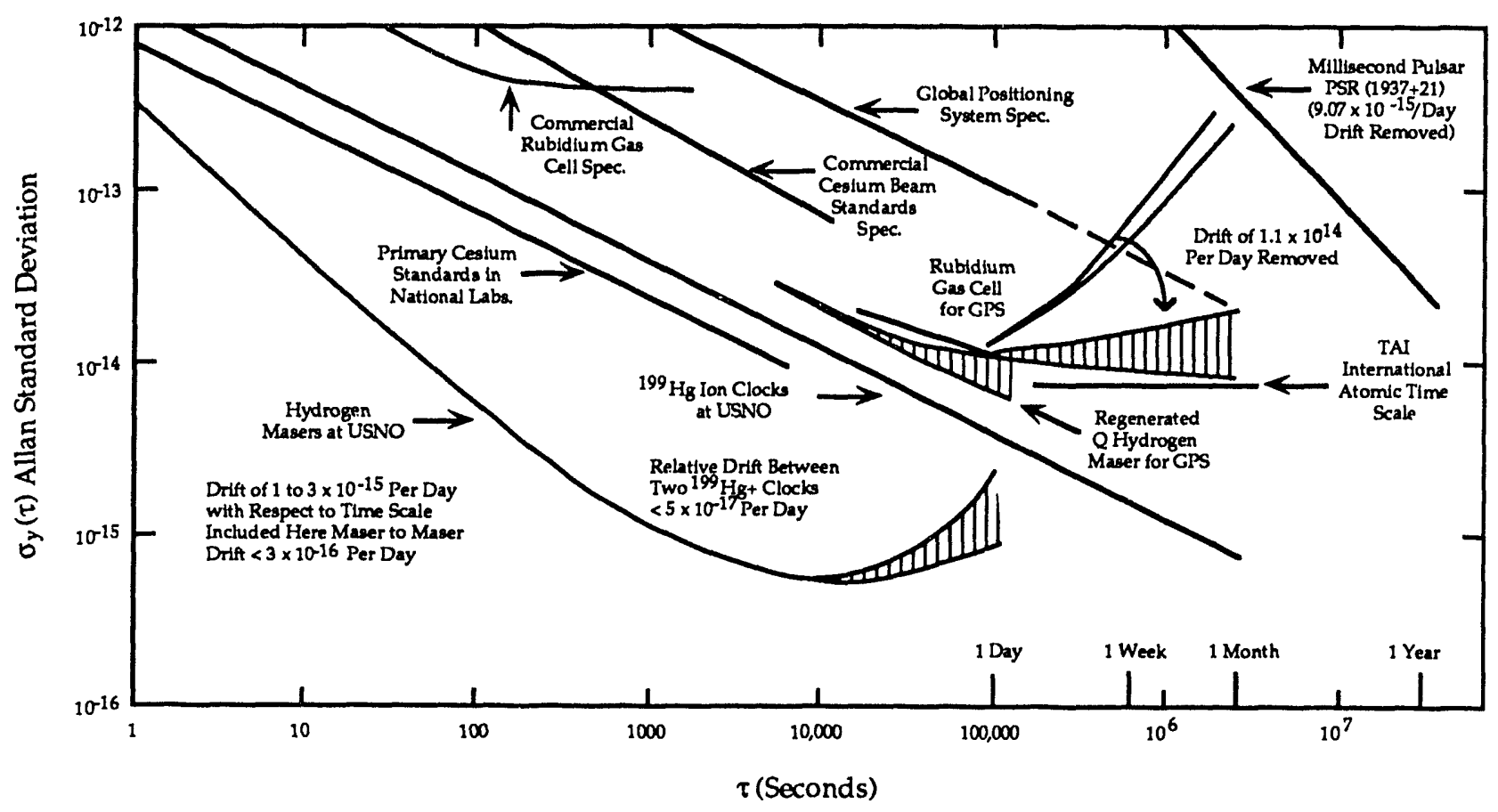

Figure II.7

Current Status of Stability of Atomic Frequency Standards 


\section{CHAPTER III \\ ATOMIC FREQUENCY STANDARDS}

\section{A. SUMMARY}

\section{Rubidium Gas Cell Standards}

The rubidium gas cell frequency standard is the least costly, most compact, and least power hungry of the three working standards now in general use. While clearly not the best performer for long-term stability and absolute accuracy, rubidium gas cell standards are ideally suited for mobile operations in vehicles under high-vibration conditions and are extensively used in military systems. Soviet researchers have devoted much effort to rubidium standards. The published literature reveals that work beginning in the late 1960s was mainly concerned with the underlying physics of the optical pumping technique used in rubidium devices. Little Soviet work on hardware appeared until the 1970s, but since then, Soviet work appears to be at the state of the art and, to date, has continued at a high level of proficiency.

Soviet researchers have had access to state-of-the-art Western rubidium standards since at least 1984, when they reported that their Ch1-69 rubidium standards were superior to Hewlett-Packard's 5065A and Rhode \& Schwartz's Effratom XSRM rubidium standards. The current Soviet production rates for rubidium standards reported by N. A. Demidov and A. A. Ul'yanov of the Gor'kiy "Quartz" Scientific Production Association (NPO) are approximately 500 units per year. Recent information obtained from the Leningrad Radiotechnical Scientific Research Institute indicates that there are four space-qualified models of rubidium gas cell frequency standards ("Reference," "Amethyst," "Onyx," and "Beryl") available for spaceborne application. Excerpts from brochures on these devices are shown in Appendix A.

The use of rubidium gas cell frequency standards clearly will increase in both the Soviet Union and the West, and the Soviet Union could enter the world market with some highly successful products. 


\section{Cesium Beam Frequency Standards}

The cesium beam atomic frequency standard originated in the West from the molecular and atomic beam work by I. I. Rabi (Columbia), N. F. Ramsey (Harvard), and J. R. Zacharias (MIT). The first commercially available cesium beam standards were developed by Zacharias in the late 1950s and were built by The National Company of Malden, Massachusetts. Development for commercial use continued at Varian Associates, and later at Hewlett-Packard Frequency and Time Systems in the United States and at Oscilloquartz SA in Switzerland.

Improvements in cesium beam devices for primary frequency standards laboratories in the United States, Canada, United Kingdom, and West Germany demonstrated their value, and led to the adoption of the cesium hyperfine resonance frequency for the definition of the System International (SI) second of time in 1967.

The commercially available cesium standards were largely the result of joint government and proprietary investment by the industry, and little information on the manufacturing technology and processing was made public. In contrast, in developing primary cesium beam standards, the Soviet Union had access to developments made in various Western primary standards laboratories where cesium beam technology was available from publications in Western journals. Soviet researchers clearly copied the essential features of these standards for their primary standards at the Physical-Technical and Radio Engineering Measurements All-Union Scientific Research Institute (VNIIFTRI). By the mid 1970s, the Soviet Union acquired several Hewlett-Packard cesium beam standards designed for the commercial market, and a number of cesium beam devices built in Switzerland using US-made cesium beam resonator tubes. At that time, some evidence of Soviet work on small-sized cesium beam tubes was reported by US visitors to the Soviet Union, and cesium beam standards are currently being developed by the Leningrad Radiotechnical Scientific Research Institute.

The development of primary standards in the Soviet Union has mostly followed Western ideas and designs, including the use of optical pumping state selection in place of conventional magnetic techniques. The theoretical aspects 
of optical pumping appear to be well developed and understood in the Soviet Union.

Discussions held with A. A. Ul'yanov and N. A. Demidov of the Gor'kiy "Quartz" NPO during their visit to the United States in November-December 1990 revealed that the development of smaller cesium beam tubes for use as secondary standards in laboratory and space applications began at Gor'kiy probably within the last 10 years, but was later abandoned. Ul'yanov and Demidov described a litany of vacuum problems that had plagued their efforts. It is clear from their discussion that the group making the vacuum envelopes for the tube and its electrical feed-through headers has much to learn about processing technology; this type of information is not readily available from dissecting a successful Western-made product.

The future for cesium beam devices is promising. Of the three commonly used frequency standards-cesium, rubidium, and hydrogen-cesium, alone, offers the possibility of generating signals of high frequency accuracy and repeatability. The cesium beam technique allows the isolation of the atom without collisional confinement, as in the rubidium gas cell and the hydrogen maser-processes that are subject to aging. Furthermore, the collision process inevitably causes a frequency shift that, in the case of rubidium, requires close control of the temperature and composition of the gas mixture in the cell. In the case of hydrogen, the confining walls cause collisional frequency shifts that are subject to variations depending on the physical configuration (on an atomic scale), as well as the contamination of the surface by chemical bonding of unwanted substances. While the atomic linewidth of cesium beam devices depends on the transit time of atoms traveling across the interaction region, and hence, on the length of the tube and the velocity of the atoms vaporized at the source, there are now optical techniques for reducing the speed of the atoms. Optical techniques, using laser sources, can be used to replace the conventional magnetic state selection and state identification methods traditionally used in beam devices. These new techniques allow improved signal-to-noise and, therefore, short-term stability. The most advanced cesium beam devices employ microprocessor-controlled modulation and synchronous detection systems that allow automatic system diagnostics and corresponding corrections. 
The most advanced Soviet cesium beam devices appear to be the spaceborne standards known as "Gem" and "Malachite;" these are discussed in Appendix A. Details on these devices are sparse; however, it is clear that the Soviet GLONASS system has successfully operated cesium devices built by the Leningrad Radiotechnical Scientific Research Institute (Gouzhva et al., 1990). The performance of these standards has improved dramatically over the past few years, and currently is as good as the cesium clocks in the GPS system. While no information on reliability is available, we know that each GLONASS satellite carries three cesium beam standards for redundancy. It is becoming clear that cesium devices will play an increasingly important role in both US and Soviet spaceborne timekeeping.

\section{Hydrogen Maser Standards}

Soviet researchers were quick to adopt the atomic hydrogen maser for their primary and working frequency standards shortly after the hydrogen maser was invented in 1959 by Kleppner and Ramsey at Harvard University. Because it is a self oscillator, the basic technology of the hydrogen maser is simpler than that of other standards using atomic resonances as frequency discriminators and requiring frequency-lock servo systems to control their quartz crystal oscillators.

In contrast to the more mature technology of rubidium and cesium standards that became commercial products, nearly all of the work done in the United States on hydrogen masers is reported in the published literature, and during the first decade of progress, the Soviet work appeared to have lagged the US work by about three years. Soviet work began in the early 1960s. By 1965, the Soviets reported a detailed replication of Ramsey's work at Harvard by a group headed by Nikolay Basov, who along with Aleksandr Prokhorov, shared the 1964 Nobel Prize for physics with Charles Townes of the United States. A strong Soviet effort continued from that time to produce a standard with a high degree of both frequency accuracy and frequency stability using the hydrogen maser. The question of hydrogen as a primary standard was settled by the 1967 redefinition of the SI second of time in terms of the hyperfine separation frequency of cesium 133. Work in the Soviet Union continued, and by early 1968, as many as 10 hydrogen masers (model Ch1-44) were produced by the Gor'kiy "Quartz" NPO. Advances continued with a 1974 version (model Ch1-70) with all-metal seals and 
an improved glass-ceramic cavity resonator. Progress in storage volume wall coatings and electronics led to the Ch1-80 maser system. Approximately 150 of these devices have been built for use in the National Time and Frequency Service throughout the European and Asiatic parts of the Soviet Union, and for very long baseline interferometry (VLBI). Soviet researchers have made extensive and successful use of automatic cavity tuning systems to remove systematic drift caused by the cavity resonator frequency.

In the West (chiefly the United States), work has concentrated on improving frequency stability over time intervals of less than one day for use in spacecraft tracking and VLBI. As Soviet work progressed and their production rate increased in the last decade, the performance gap has closed and the Soviet maser performance is fully comparable to that of the best US-made masers. The Soviet production rate is at least 10 times that of the West.

Soviet research and development efforts involve more than 10 times as many scientists and engineers as in the rest of the Western world, combined. While the Soviets have not made many innovations, one stands out as highly significant in that it involves the process of unperturbed hydrogen storage, which is the heart of the technique. Soviet polymer chemists have developed a far superior fluorocarbon surface capable of five to 10 times more effective storage than any used in the West.

Soviet engineers have designed hydrogen masers with reduced weight and lower power requirements using the technology developed in the United States for the 1976 space probe relativity test. Small, portable masers have also been developed for time transfer clocks that have been successfully used in comparisons with the Paris Observatory and Cuba.

Increased worldwide use of hydrogen masers as stable "flywheel oscillators" in timekeeping centers is expected. This technique has proven to be valuable in the operation of hydrogen masers at the US Naval Observatory. Hydrogen masers continue to be the most stable oscillators for intervals from about 1 second to one day. They are used exclusively in the National Aeronautics and Space Administration's (NASA) space tracking operations run by the Jet Propulsion Laboratory, in radio astronomical VLBI measurements, and in high-preci- 
sion physics experiments. There are plans to use spaceborne hydrogen masers for timekeeping applications, VLBI, and for testing gravitation and relativity theory.

A comparison of the performance of Soviet- and US-made hydrogen masers was conducted at the Smithsonian Astrophysical Observatory (SAO) in November-December 1990 (the performance of the Soviet clocks is reviewed in Appendix A). The tests revealed that the electronic and mechanical engineering, workmanship, and performance of the Soviet masers are close to the state of the art. Soviet hydrogen masers are now actively being marketed worldwide at prices far below those of the West. Both the high production rate and the need for Western currency have certainly been factors in this enterprise.

There is room for further US work on hydrogen masers in research, development, and manufacturing, as the expansion of applications in space and on Earth accelerates with the advance of the technology.

\section{B. INTRODUCTION}

In a 1970 paper entitled "History of Russian Metrology-Old Russian Time Units," N. A. Shostkin acknowledges that, starting in the first decade of the 15th century and continuing through the 16th and 17th centuries, it was Western technology that opened up a new era in Soviet time measurement. The pattern established by the introduction, in 1700, of mechanical clock technology, and the subsequent recognition and adoption of Western definitions of time and time interval measurement, seem to have set the precedent for recent timekeeping concepts and developments in the Soviet Union.

In the 1960s, extensive Western efforts on hydrogen, cesium, and rubidium received much attention from Soviet scientists and engineers. The current Soviet technology base reveals strong parallels to Western (in particular, the United States) development paths and technical concepts. In the 1970s and 1980s, the Soviet Union moved forward in atomic timekeeping technology due to the detailed technical road maps provided in Western publications showing what worked well and what did not. The Soviet literature reveals no new developments in either cesium or rubidium clocks. 
In 1960, five years after initial publication, the Soviet Union had a translated version of Norman Ramsey's Molecular Beams which, as the most definitive work in atomic timekeeping, served as the Soviet primer in this area. Researchers around the world have used this book to support work in hydrogen, cesium, and rubidium. Ramsey's personal interest in. hydrogen led to many additional publications on that subject. As discussed eisewhere in this report, the Soviet hydrogen maser effort led to the Soviet push into modern-day precision timekeeping technology. Ramsey's book provided the Soviets with the history, technology, and direction for fruitful research in masers.

In a paper by Baydakov et al. (1973), the authors presented a blueprint for Soviet research and technical focus that was followed for the next 10 years. In the 1970 s, a series of papers followed which confirmed the Soviet technical focus to establish teams and facilities to enable them to achieve capabilities comparable to Western precise time and time interval capabilities. The timing of this effort appears to coincide with:

- The Soviet realization of a need for improved navigational and communication capabilities and accuracies in both space and military systems. These needs were again stated at a later time by Pushkin et al. (1989).

- The recognition that the Soviet Union did not have its own capability to field a national cesium standard when the rest of the world had already agreed (in 1967) upon the cesium atom for the basic definition of time and frequency.

The volume of papers in the 1970s and 1980s indicates a strong ert on hydrogen-perhaps influenced by Soviet graduate students having had access to a Russian-language version of Ramsey's Molecular Beams during their training in the 1960s, and by the prestige and support which must have been generated by Basov and Prokhorov sharing the 1964 Nobel Prize for masers with Townes. During this time, most of the in-depth papers on precise time and time interval in the United States (and PTB, or Physikalish-Technische Bundesanstalt, the West German NBS) were hydrogen oriented, such as those pubiished by Ramsey 
and his students (Kleppner, Vessot, Crampton, Novak, Goldenberg, and Peters). These papers and technical conferences openly detailed the progress of those working on hydrogen maser technology. These Western reports were rich in technical detail because support for these efforts came from public-domain sources such as universities, NASA, the National Bureau of Standards (NBS), and the Office of Naval Research (ONR), and sponsors like to see papers supporting significant advances which credit and justify their commitment and expenditures.

In the past, and continuing today, there has been virtually no commercial interest in developing hydrogen masers in the United States. Market surveys in the United States have continued to come up empty. Thus, no company has invested significant funds for research, development, or production of hydrogen masers since Varian Associates in the 1960s. It is surprising that in the United States the hydrogen maser is still in a development phase and has not achieved large-scale usage like that of cesium and rubidium instruments, which has driven their commercial advancement. This is not the case in the Soviet Union.

The Soviat position in hydrogen masers reflects their strongest, most balanced technology. The hydrogen maser is used widely across the Soviet Union and in such remote or satellite locations as Havana, Cuba. Many of the weaknesses (and strengths) revealed in the extensive Soviet literature on maser research have direct application to all other standards. However, the Soviet literature contains relatively few technical papers on cesium and rubidium clocks, despite the fact that the basic hydrogen maser technology drivers obviously mastered by Soviet researchers (such as quantum physics, vacuum technology, RF chains, synthesizers, and crystal oscillators) are common necessities to cesium and rubidium clocks and to frequency standards.

It is plausible that the Soviet development of cesium (and to a lesser extent, rubidium) may have been hampered by: (i) a lack of incentive for knowledgeable engineers and scientists to work these technologies (with a Nobel Prize for masers, hydrogen masers probably were the politically correct technology), and (ii) too few Western sources which revealed details of the special techniques of either cesium or rubidium. 
The literature suggests that the initial Soviet efforts on cesium technology focused on the development of laboratory cesium equipment which was remarkably similar to the national standards of the United States (NBS) and (West) Germany (PTB). Prior to development of the Soviet national cesiums MCS-1, MCS-2, and MCS-3, the major Soviet experience with cesium was the use of several Hewlett-Packard 5061 (HP-5061) commercial cesium clocks, and the evaluation and acquisition of a number of Swiss cesium standards whose cesium tube originated in the United States (II'in et al., 1979). In the middle 1970s, NBS members visiting the Soviet Union reported seeing a small Soviet-made cesium beam tube which was not operational in a clock. No reports of such an endeavor were found in the Soviet literature.

With the 1967 adoption of the definition of the second as 9,192,631,770 cycles of the cesium atom, it was appropriate for the State Time and Frequency Service to start a large machine effort at VNIIFTRI.

Development of atomic clocks began in the early 1950s and originally was based on the atomic and molecular beam experiments that flourished with the availability of World War II microwave technology. The idea that it was possible to interrogate atoms of alkali metals such as cesium, sodium, and rubidium (previously used in atomic beam clock experiments by diluting them in vapor form in mixtures of inert gases confined in light-transparent cells) revolutionized atomic clocks because it allowed them to be made more compactly and less expensively. In 1960, the process of confining atoms in a relatively unperturbed manner was further advanced by the invention of the atomic hydrogen maser, which found almost immediate application in VLBI radio astronomy as a combined clock and high-stability local oscillator. The demand for atomic clocks increased as they became more reliable and useful for terrestrial and space navigation systems; special devices were developed for particular applications. The recent development of national time and frequency standards based on new coniinement methods involving the levitation of atoms by laser beams and the long-term trapping of single ions, combined with the feasibility of worldwide time synchronization at the subnanosecond level, should lead to even broader application of frequency and time as the basis of metrology. 
Since both cesium molecular beam and rubidium gas cell standards use similar servo techniques to control the frequency of quartz oscillators, they are discussed here together. A schematic of a frequency-lock servo is presented in Figure III.I. A voltage-controlled crystal oscillator drives a frequency synthesizer that produces a microwave signal at the appropriate atomic hyperfine transition resonance frequency which is applied to the atom. To determine the center frequency of the atom's response to this stimulus, the frequency must be varied, and the level of the response must be observed by a detection system. Frequency modulation of the microwave signal is usually done by phase modulating a lowfrequency stage of the frequency multiplier chain. The modulated microwave signal sweeps, or steps, back and forth across a small part of the resonance near its center. The response is in the form of an alternating current (AC) signal at the modulation frequency, the magnitude and phase of which are proportional to the departure from the precise center frequency of the resonance. By comparing the output signal with the modulation signal in a synchronous detector, a direct current (DC) voltage is obtained whose magnitude and sign correspond to the location of the microwave signal on the resonance. This signal is used to control the frequency of the quartz oscillator. Because the signal contains noise from the resonator (such as shot noise from the arrival of cesium atoms at the ionizer, or photon noise from the rubidium photocell detector), as well as the intrinsic noise from frequency fluctuation in the quartz-crystal-oscillator frequency synthesizer, the combination of these two noise sources must be optimized. This is done by placing a filter in the frequency controlling voltage of the servo loop so that the high-frequency noise contributions of the resonator do not corrupt the output at the oscillator, and the new frequency variations at the oscillator are corrected by the atomic resonator.

The atomic clock is at the heart of the present era of metrology based on frequency and time. This section describes and assesses Soviet progress in the development and manufacture of rubidium gas cell, cesium beam, and atomic hydrogen maser clocks, and the electronic systems that support their frequency and time metrology. Again, the possibility of worldwide time synchronization at the subnanosecond level should lead to even broader applications of frequency and time as the basis of metrology. 

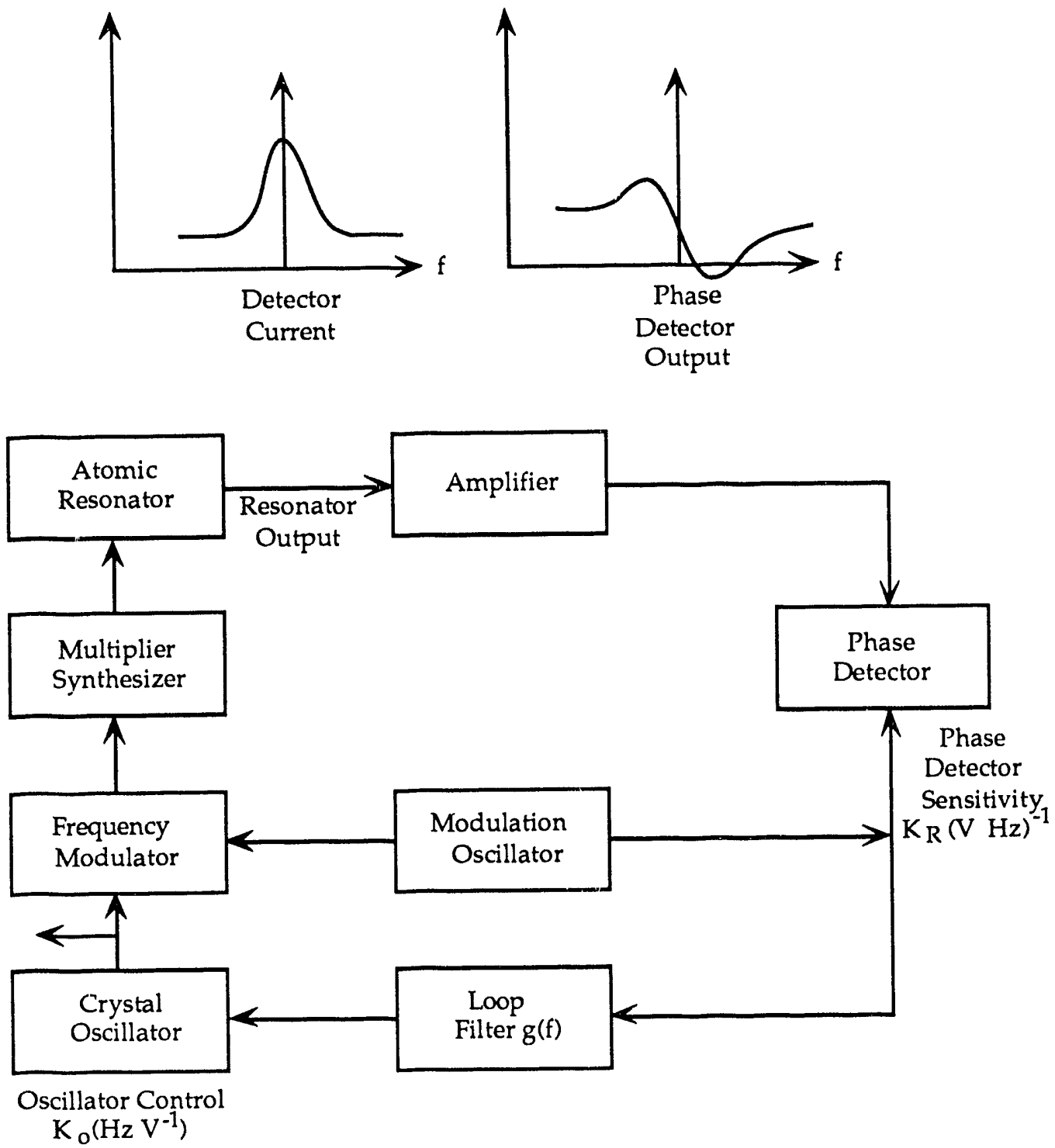

Figure III.1

Schematic of a Frequency-Lock Servo

\section{Rubidium Gas Cell Resonators}

The rubidium gas cell resonator uses optical pumping to produce an unbalanced population of atoms in the ground state of rubidium 87. Quantum transitions are then stimulated by applying a microwave resonance signal, and the degree of resonance is monitored by observing the intensity of the optical-pumping light passing through the cell. Figure III.2 is a schematic of the rubidium gas cell frequency discriminator. 


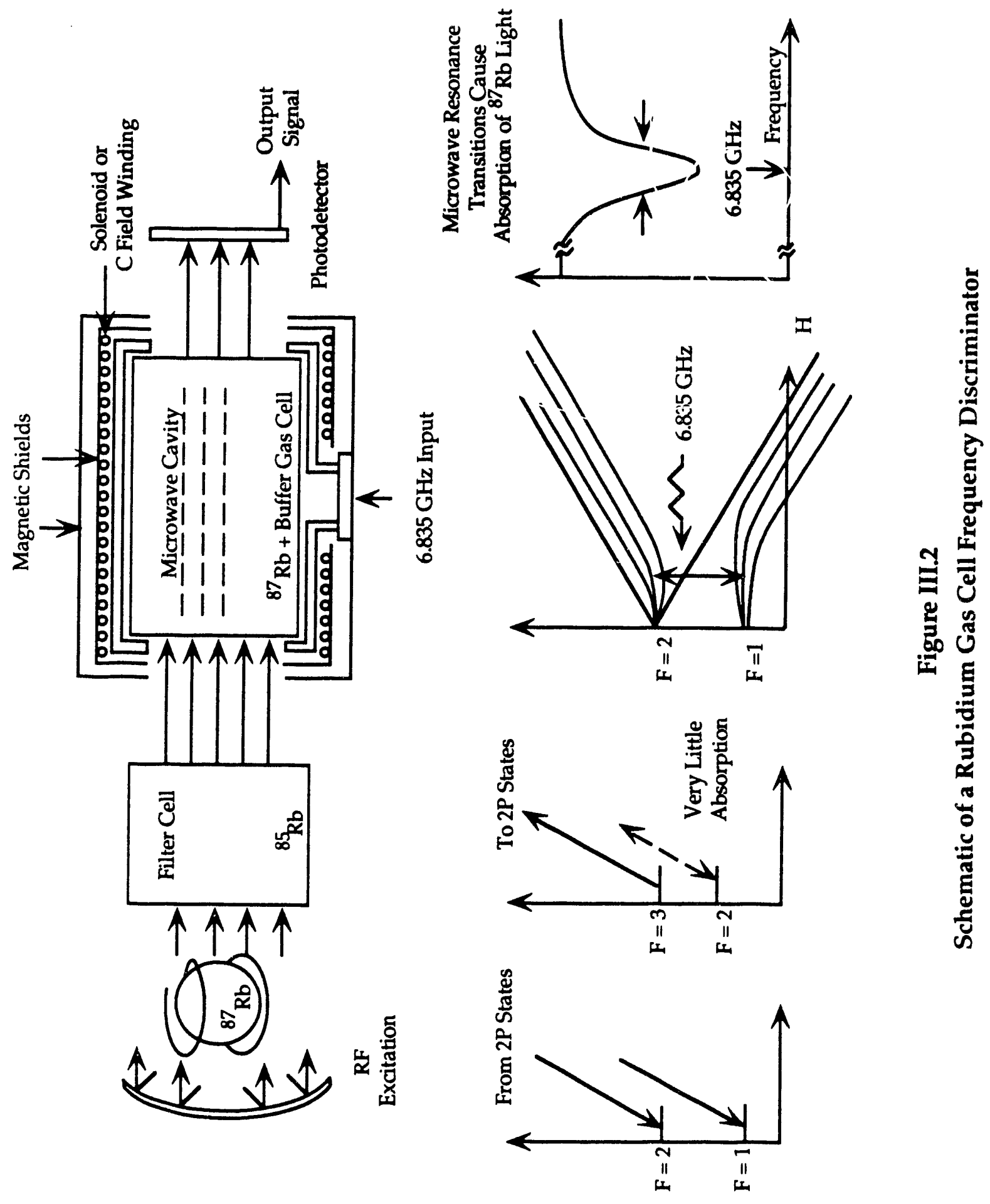

III-12 
The process of optical pumping in the rubidium 87 resonator is done by illuminating the atoms in the ground state with narrow-band light energy to induce quantum transitions to higher energy levels. The frequency of the light is chosen to depopulate the lower ground-state hyperfine level while leaving the upper hyperfine level undisturbed. By applying microwave energy at the proper hyperfine resonance frequency, atoms in the upper level make transitions to the lower level and thus repopulate it, causing the pumping light to be absorbed. The decrease in the pumping light transmitted through the resonance cell provides a method for detecting the resonance.

The pumping light is obtained from a cell containing rubidium 87 that is excited by radio frequency ( $R F$ ) power to provide ionization of the inert gas. Light from this plasma discharge contains the proper spectral lines due to transitions from upper quantum states to the ground-state hyperfine levels, shown as $\mathrm{F}=2$ and $\mathrm{F}=1$ in Figure III.2, where $\mathrm{F}$ is the total angular momentum of the atom. In order to depopulate only the lower ground-state hyperfine level of the rubidium atoms in the microwave cavity, the light first must be filtered to remove the optical components involving the upper ground-state levels. This is done by using a highly selective optical filter composed of rubidium 85 atoms mixed with an inert gas in a separate transparent cell. The optical transitions of the rubidium 85 isotope nearly match those of the upper hyperfine level of rubidium 87, while the corresponding lower hyperfine-level transitions of rubidium 85 and rubidium 87 do not match. As a result, the light emerging from the filter cell will primarily cause energy-absorptive transitions from the lower hyperfine levels of rubidium 87 in the microwave cavity.

The overlap of the desired $\mathrm{F}=2, \mathrm{~m}_{\mathrm{F}}=0$ to $\mathrm{F}=1, \mathrm{~m}_{\mathrm{F}}=0$ transition with other transitions is removed by applying a weak, homogeneous magnetic field (the so called " $C$ field") throughout the cell ( $m_{F}$ is the component of F parallel to the magnetic field). The second-order effects of variations in the magnetic fields are given by $\Delta \mathrm{f}_{\mathrm{m}}=573 \mathrm{~B}^{2}$. Because the absorption resonance can involve a large number of atoms, these devices have a strong signal-to-noise ratio and therefore have good frequency stability in the short term (10 to 1000 seconds). The random arrival of photons produces a white noise power spectral density of the frequency fluctuations of the quartz crystal oscillator controlled by the electronic system. 
The atoms in the resonance cell located in a microwave cavity are kept relatively isolated from each other by diluting them with an inert buffer gas (a mixture of argon and neon) that keeps the atoms in a relatively unperturbed condition. In this manner, the linewidth of the microwave resonance transitions can be narrowed to about $200 \mathrm{~Hz}$. This narrowing is accompanied by substantial systematic frequency shifts that are sensitive to both temperature and gas pressure in the cell. It is possible to find combinations of gases that have compensating negative and positive thermal and pressure shifts to the resonance frequency in order to obtain a mixture which results in an environmentally stable resonance frequency.

Frequency instability in the rubidium gas cell resonator is caused by variations in optical pumping light intensity and the spectral distribution of this light, temperature and barcmetric pressure changes, chemical interactions, and many other causes. It is these variables that place rubidium in the "secondary standard" category. Despite these problems, the simplicity of the resonator and its excellent signal-to-noise properties have been strong incentives for the development of small and relatively inexpensive frequency standards with excellent short-term frequency stability.

\section{Cesium Beam Frequency Standards}

Cesium beam standards are based on traditional techniques developed in the 1930s for studies of molecular and atomic beams. Atoms in a beam traveling in vacuum behave as free, non-interacting particles. Alkali atoms have magnetic dipole moments that, according to their magnetic hyperfine state, are determined by the relative orientation of the magnetic dipoles of the outer shell (valence) electron and the nuclear magnetic dipole. The hyperfine states of the atoms can be analyzed according to their trajectories through strongly inhomogeneous magnetic fields. By inducing transitions between hyperfine states of atoms with microwave signals at a precise resonant transition frequency, the detected change in the state of the atom can be used as a frequency discriminator capable of resolving millihertz at gigahertz frequencies. The cesium beam standard is the primary standard of time and frequency because it is least susceptible to envirominental or chemistry effects. 


\section{Cesium Beam Resonators}

The cesium beam resonator is totally enclosed in a high vacuum envelope. This device is shown schematically in Figure III.3. At the left side, atoms are thermally evaporated from a supply of natural cesium 133 in an oven and emerge into a vacuum system in a highly collimated beam walich then enters the first state-selector magnet. A highly inhomogeneous magnetic field deflects the atoms according to their magnetic moment, as shown in Figure III.3. Atoms in the $F=4, m_{F}=+4$ to $m_{F}=-3$ states (labeled by the symbol "4") are deflected toward stronger fields. The atoms then proceed into a magnetically shielded interaction region containing a two-section cavity which produces a microwave magnetic field parallel to a weak DC magnetic field ( $C$ field) established within the magnetic shield by a coil. The DC field provides a quantizing axis for the atoms and orients them so that transitions between similar $m_{F}$ levels $\left(\Delta \mathrm{m}_{F}=0\right)$ are stimulated and $\Delta \mathrm{m}_{F}= \pm 1$ transitions are suppressed. The DC field also splits the states sufficiently to prevent overlapping of undesired transitions at frequencies near the desired "clock" frequency, which is produced by the $\mathrm{F}=3, \mathrm{~m}_{\mathrm{F}}=0$ to $\mathrm{F}=4, \mathrm{~m}_{\mathrm{F}}=0$ transition. The two-section Ramsey RF cavity applies two separated but synchronous pulses of microwave field to each of the traversing atoms as they proceed down the tube and produces a frequency response in the atomic transition pattern analogous to that of an optical two-slit diffraction process. The width at half-maximum of the central lobe of this pattern is approximately $t / 2$ $(\mathrm{Hz})$, where $\mathrm{t}$ is the time of flight through the resonator arms. The resonance of the Ramsey resonator is unperturbed by Doppler shifts from atoms interacting with the running waves that could occur in a single interaction cavity along the beam. In commercial practice, resonator lengths range from 17.5 to 7.5 centimeters. These provide Ramsey resonance widths of 360 to $1,300 \mathrm{~Hz}$. 

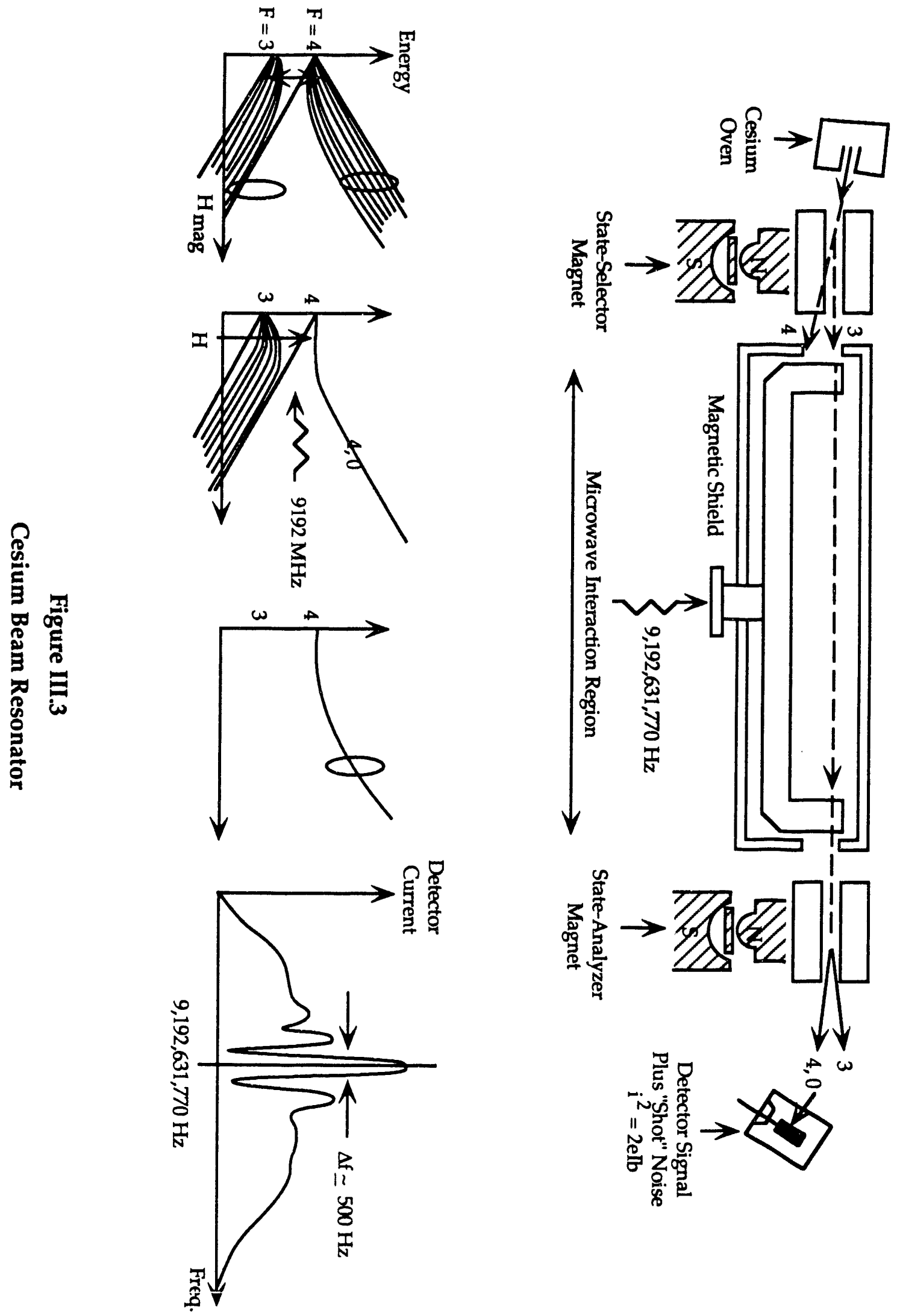

III-16 
Atoms that have been exposed to the microwave field of proper frequency and intensity will emerge from the interaction region(s) of Figure III.3 in the $\mathrm{F}=4, \mathrm{~m}_{\mathrm{F}}=0$ state, and are sorted from the other atoms in $\mathrm{F}=3$ by a permanent state-analyzer magnet similar to the previous state-selector magnet. These atoms are thermally ionized when they land on a heated ribbon of metal, which has a work-function substantially greater than that of cesium. The ions are then thermally emitted as positive cesium ions, accelerated through a mass spectrometer (to separate them from ions of other species emitted from the hot ribbon), and then further accelerated to the first dynode of an electron multiplier, where their current of about $10^{-13} \mathrm{~A}$ is amplified to the tens of nanoamperes of useful signal current necessary to drive the electronic circuitry of a clock.

The output current depends strongly on the exact frequency of the microwave excitation and is greatest at the cesium resonance. This is the basis for the frequency discriminator.

The chie noise mechanism of the output signal is shot noise due to the arrival of individual atoms at the detector. This noise has a "white" or power spectral density that causes a "white" spectral density of frequency fluctuations in the output of the frequency-lock servo system.

\section{CESIUM ATOMIC CLOCKS}

\section{Summary}

The Soviet literature on cesium beam technology indicates a tracking of Western publications follow d by the Soviet realization of similar equipment. However, this realization is delayed by a few years and lacks the sophistication and performance of the original work. Most Soviet articles on cesium indicate knowledge that originated in Western laboratories which reported on national standards and their performance. Soviet cesium technology has advanced in this area, as exemplified by the CS-1, CS-2, and CS-3 MMC/STFS developments at VNIIFTRI.

Although the large Soviet cesium units might have provided the Soviet Union with a starting point to advance both its national and industrial (or tacti- 
cal) standards, it appears that technical progress was severely hampered by a lack of industrial support technologies necessary to the art and science of cesium clocks, such as magnetic shield fabrication, solid-state electronics, vacuum technology, and computer-aided measurement systems. In addition, Western cesium manufacturers publish few detailed technical papers because the competitive nature of the cesium business has made an issue of corporate proprietary rights and the need for secrecy. In this particular area of timekeeping technology, the Soviet Union must start at first principles. The Soviet system also did not provide an innovative environment supported by the sophisticated industrial know-how necessary for the development of instruments requiring the balanced integration of physics, mechanics, and electronics. The Soviet Union now appears to be 'aking significant steps to rectify these deficiencies.

\section{Discussion of Soviet Work}

The published literature indicates that the Soviet cesium effort has followed the same developmental path as the rest of the world-only 10 years later. Abashev et al. (1973) reported studies of hot wire ionizer materials that focused on methods of achieving lower additive noise from the ionization process through the use of cleaner, higher purity materials produced in special laboratory facilities. They cited the work of both J. George and Richard Lacey of the United States in 1963 and 1967. In 1974, Abashev and coworkers reported on work with tungsten, tantalum, and niobium, and favored the use of single-crystal detectors to solve the problem of impurity noise bursts from hot wire ionizer detectors (Abashev et al., 1974). In the United States, the additive detector noise problem was solved some 10 years earlier through the use of a mass spectrometer incorporated into the cesium physics package; this was found to be necessary by most researchers in industry. Experimenters in the United States found that additive noise was virtually independent of material purity (that is, tungsten, tantalum, and niobium) since significant hot wire contamination occurs from both the assembly process and the operating vacuum environment of the apparatus.

Papers on cesium beam velocity distribution by Abashev (1972) and Kurakin (1972) report that beam intensity characterizations followed the traditional approach (though 10 years later) toward the realization of cesium machines with reasonable performance expectations. 
It is interesting that Abashev et al. examined the potential of thallium atomic beam technology in 1972, again just about 10 years behind the operational thallium tubes built earlier by Jacques Bonanomi at the Neuchâtel Observatory in Switzerland, and by Richard Lacey at Varian Associates in Massachusetts.

Soviet efforts to produce a US/NBS-style dipole optics cesium standard began in the early 1970s and resulted in MCS-1. The first cesium results on MCS-1 reportedly were obtained in 1976, and work continued (Yelkin et al., 1983) to improve both the cesium tube and its supporting electronics systems. The lack of good magnetic shielding designs (or, more realistically, executions) was reported; this certainly is consistent with earlier-reported problems on hycrogen maser shielding. Next followed the State Primary Time and Frequency Standard's MCS-2, a hexapole optics machine patterned after the (West) German PTB CS-1 and CS-2 national standards. The MCS-2 machine was the first Soviet cesium device to use a hexapole deflecting magnet system that was similar to the (West) German PTB multipole cesium which first produced data in 1978. Frequency stability of this apparatus, which utilized the (very) same electronics as MCS-1, was measured to be $\cdot 10^{-12} / \sqrt{\tau}$. When MCS-3 operated in March 1984, it was a longer machine set up with dipole optics that were similar to MCS-1. This unit exhibited a much improved signal-to-background ratio (5:1) than previous units and utilized a "C-field" generating design originally used by the Laboratoire Suisse de Recherches Horlogères (Neuchâtel, Switzerland) in one of their cesium devices circa 1970.

After these state standards received modifications to improve magnetic shielding to apply temperature control to the microwave cavities, to improve vacuum $b$; enclosing the beam in a copper tube, and to apply more sophisticated pumping schemes, the accuracy of the three State frequency standards (MCS-1, MCS-2, and MCS-3) was estimated to improve to a uniform 10-13. As reported by the Soviet Union to the Consultative Committee for the Definition of the Second (CCDS) in March 1989, MCS-1 and MCS-2 currently are the only "legal" State Primary Time and Frequency Standards. 
In 1986, another state cesium standard was developed at VNIIFTRI on the theory that either dipole or hexapole deflection optics could be employed or, with the deflecting magnet system removed, it could be used for experiments on an optically pumped cesium device (Abashev et al., 1986).

MCS-3 and MCS-4 remain experimental, with MCS-3 being three times poorer than MCS-1 or MCS-2. MCS-4 is a demountable experimental unit suitable for use as either a cesium apparatus using magnetic state selection or for cesium optical pumping experiments.

The Soviet literature is rich with both theoretical and laboratory work on optical pumping using cesium as well as the required laser technology associated with optical pumping. It is clear from the literature that the Soviet Union has made a commitment to be a leader in this field. This focus promises performance improvements in both short-term stability and in accuracy. Until significant engineering problems can be solved, this technology would be best focused at the national standard level (at VNIIFTRI), where the environment can be carefully controlled (for example, temperature, vibration, and pressure). The Soviet Union has entered this field in a timely manner and, in addition to its own efforts, it will be able to draw upon the excellent work of laboratories in France, Canada, Italy, and the United States. The ability to produce or gain access to a source of stable, solid-state lasers will be a key requirement for Soviet success in cesium optical pumping.

It is clear from both the published literature and from data comparison that the West has provided the Soviet Union with an excellent starting point for VNIIFTRI's development of cesium technology. Table III.1 provides a historical time/technology comparison of cesium standards.

David Allan's September 1989 visit to VNIIFTRI supports an underlying concept found in the literature of a civilian/military split in Soviet time and frequency research/development/production. Work at VNIIFTRI is heavily oriented toward the operation of hydrogen masers, both fixed station and portable. There is a secondary focus on the design, construction, and maintenance of the four national cesium units, co-mingled with increasing emphasis on the further development of opticaily pumped cesium. 
Table III.1

TABULATION OF WESTERN/SOVIET NATIONAL CESIUM STANDARDS

\begin{tabular}{|c|c|c|c|c|c|c|c|c|c|c|}
\hline Designator & Circa & $\begin{array}{c}\text { State } \\
\text { Selectors }\end{array}$ & $\begin{array}{c}\text { Length } \\
(\mathrm{cm})\end{array}$ & $\begin{array}{c}\text { Line- } \\
\text { width } \\
(\mathrm{Hz}) \\
\end{array}$ & $\begin{array}{l}\text { Back- } \\
\text { ground } \\
\text { Current } \\
\text { (pA) }\end{array}$ & $\begin{array}{c}\text { Signal } \\
\text { Current } \\
\text { (pA) }\end{array}$ & $\begin{array}{l}\text { Number/ } \\
\text { Shields }\end{array}$ & $\begin{array}{l}\text { Stability } \\
\left(.10^{-12} / \sqrt{8}\right.\end{array}$ & Accuracy & Remarks \\
\hline РTB CS-1 & 1964 & Hexapole & 80 & 63 & - & 10 & 4 & 10 & $3 \times 10^{-14}$ & $\begin{array}{l}\text { Used as } \\
\text { dock }\end{array}$ \\
\hline NBS 4 & 1967 & Dipole & 52.4 & 200 & 1 & 11 & 2 & 2 & N/A & $\begin{array}{l}\text { Used as } \\
\text { dock }\end{array}$ \\
\hline NBS 5/6 & 1970 & Dipole & 375 & $45 / 26$ & $5 / 1$ & $1 / 5$ & 3 & 1 & $1 \times 10^{-13}$ & \\
\hline MCS-1 & 1976 & Dipole & 65 & $125 / 250$ & - & - & - & 3 & Sov. ref. & $\begin{array}{l}\text { Beam size } \\
8 \times 2 \mathrm{~mm}\end{array}$ \\
\hline MCS-2 & 1978 & Hexapole & 100 & 110 & 50 & $5 / 10$ & - & 5 & $\begin{array}{l}1 \times 10^{-13} \\
-0 . \text { (Ref.) }\end{array}$ & \\
\hline PТB CS. 2 & & Hexapole & 79 & 60 & - & 25 & 4 & 3.7 & $1.5 \times 10^{-14}$ & \\
\hline MCS-3 & 1984 & Dipole & 194 & 50 & 1 & 5 & $3-4$ & 5 & $1 \times 10^{-13}$ & \\
\hline MCS-4 & 1985 & $\begin{array}{l}\text { Mag. or } \\
\text { optical }\end{array}$ & 105 & 105 & 30 & 5 & - & & $8 \times 10^{-14}$ & \\
\hline NBS 7 & 1988 & Optical & 55 & 65 & - & - & 3 & TBD & TBD & In test \\
\hline
\end{tabular}

Recent Soviet data sheets on cesium and rubidium describe small, portable standards, supposedly of Soviet manufacture. This may indicate some manufacturing success in producing compact physics/electronics packages in both cesium and rubidium products. This area should be followed closely as it indicates a shift in cesium emphasis from laboratory standards to working field instruments.

A 1982 data sheet announces the commercial availability of a Soviet industrialized rubidium standard, and a 1988 data sheet announces a $18.9 \times 19.7 \times$ 14.9-inch cesium standard ("Nauka 88"). This package size dictates the Soviet use of a cesium physics package whose maximum dimension must be limited to 19 inches and, more likely, the size of existing Western tubes which are 13 to 16 
inches in length. Without further performance data, one can only speculate where these cesium physics packages originated.

In light of Western visits to VNIIFTRI and the GLONASS and military use of both cesium and rubidium clocks, it would appear that there is a second (and perhaps more important) "center of excellence" in the Soviet Union, most probably located in Gor'kiy. ${ }^{1}$ UK researcher Peter Daly reports that recent data taken from onboard clocks controlling the GLONASS satellites suggest that an improvement in orbital clock stability has been achieved. ${ }^{2}$ Table III.2 presents Daly's view of the GLONASS ensemble and compares GLONASS (Soviet) and GPS (US) atomic clock performances aboard international navigation satellite systems.

If one recognizes that ensembles of rubidium clocks and GLONASS clocks are technically controlled (and built?) by the military-oriented Soviet Space Command, the second center is probably the Gor'kiy "Quartz" NPO (Kalaytanov and Boriskin, 1984).

Soviet researchers at Gor'kiy "Quartz" have expressed surprise that Western industrialized cesium clocks have achieved life expectancies of five to 20 years. The nature of the Soviet response to cesium life expectancy leads to speculation that their smaller industrial clocks may have developmental life-expectancy limitations that could have been overlooked because Soviet researchers were experienced in working with a large volume physics package (for example, NBS/PTB/ VNIIFTRI-type standards). It will be revealing to follow Peter Daly's measurements of GLONASS life expectancy versus clock signatures; this might indicate whether the physics package is of Soviet or US origin.

1 The city of Gor'kiy has recently resumed its pre-1932 name, Nizhniy Novgorod. As the research for this report predates this recent change, the Gor'kiy name will be retained throughout the report.

2 P. Daly, "Current GPS/GLONASS Time Reference and UTC," Proc. 22nd Annual Precise Time and Time Interval (PTTI) Applications and Planning Mtg., December 1990, p. 87 (in press).

P. Daly, "Review of GLONASS System Characteristics," Proc. 1990 Institute of Navigation Conf., September 1990, 267-275. 


\begin{tabular}{|c|c|c|c|}
\hline \multicolumn{4}{|c|}{$\begin{array}{l}\text { Table III.2 } \\
\text { NASS (GL) AND NAVSTAR (PRN) CLOCK } \\
\text { FLICKER FLOOR }\end{array}$} \\
\hline Device & Launch Date & $1 /$ f Floor $\times 10^{13}$ & Clock Type \\
\hline $\begin{array}{l}\text { GL-23 } \\
\text { GL-24 } \\
\text { GL-28 } \\
\text { GL-29 } \\
\text { GL-30 } \\
\text { GL-34 } \\
\text { GL-35 } \\
\text { GL-36 } \\
\text { GL-37 } \\
\text { GL-38 } \\
\text { GL-39 } \\
\text { GL-40 } \\
\text { GL-41 } \\
\text { GL-42 } \\
\text { PRN-06 } \\
\text { PRN-08 } \\
\text { PRN-09 } \\
\text { PRN-11 } \\
\text { PRN-13 } \\
\text { PRN-12 } \\
\text { PRN-03 }\end{array}$ & $\begin{array}{c}\text { 16 Sep } 1986 \\
\text { 16 Sep } 1986 \\
\text { 16 Sep } 1987 \\
\text { 16 Sep } 1987 \\
\text { 16 Sep } 1987 \\
\text { 21 May } 1988 \\
\text { 21 May } 1988 \\
\text { 21 May } 1988 \\
\text { 16 Sep } 1988 \\
\text { 16 Sep } 1988 \\
\text { 16 Sep } 1988 \\
\text { 10 Feb } 1989 \\
\text { 10 Feb } 1989 \\
\text { 1 Jun } 1989 \\
\text { 6 Oct } 1978 \\
\text { 10 Dec } 1978 \\
\text { 28 Apr } 1980 \\
\text { 14 Jul } 1983 \\
\text { 13 Jun } 1984 \\
\text { 8 Sep } 1984 \\
\text { 9 Oct } 1985\end{array}$ & $\begin{array}{c}2.3 \\
2.7 \\
1.0 \\
5.7 \\
11.2 \\
4.1 \\
3.6 \\
1.4 \\
0.6 \\
0.6 \\
3.1 \\
4.8 \\
3.3 \\
0.5 \\
1.6 \\
140.1 \\
1.4 \\
0.7 \\
0.7 \\
0.7 \\
1.5\end{array}$ & $\begin{array}{l}\text { Cesium } \\
\text { Cesium } \\
\text { Cesium } \\
\text { Cesium } \\
\text { Cesium } \\
\text { Cesium } \\
\text { Cesium } \\
\text { Cesium } \\
\text { Cesium } \\
\text { Cesium } \\
\text { Cesium } \\
\text { Cesium } \\
\text { Cesium } \\
\text { Cesium } \\
\text { Rubidium } \\
\text { Quartz } \\
\text { Rubidium } \\
\text { Cesium } \\
\text { Cesium } \\
\text { Cesium } \\
\text { Rubidium }\end{array}$ \\
\hline
\end{tabular}

Source: Ivanov et al., 1990

\section{Projections for the Future}

The future for cesium beam devices is promising. Of the three commonly used frequency standards-cesium, rubidium, and hydrogen-cesium, alone, offers the possibility of generating signals of high frequency accuracy and repeatability. The cesium beam technique allows the isolation of the atom without collisional confinement, as in the rubidium gas cell and the hydrogen maser-processes that are subject to aging. Furthermore, the collision process inevitably causes a frequency shift that, in the case of rubidium, requires close control of temperature and compensation of the gas mixture in the cell. In the case of hydrogen, the confining walls cause collisional frequency shifts that are subject to 
variations depending on the physical condition (on an atomic scale), as well as the contamination of the surface by chemical bonding of unwanted substances. While the atomic linewidth of cesium beam devices depends on the transit time of atoms traveling across the interaction region, and hence, on the length of the tube and the velocity of the atoms vaporized at the source, optical techniques are now available for reducing the speed of the atoms. Other optical techniques are possible that use laser light to perform optical pumping among the 16 hyperfine levels of cesium and permit the concentration of the beam of atoms into a single hyperfine state. This technique can be used to replace the conventional magnetic state selection methods traditionally used in beam devices. These new applications of lasers allow improved signal-to-noise and, therefore, short-term frequency stability. The most advanced cesium beam devices employ microprocessor-controlled modulation and synchronous detection systems that make possible automatic system diagnostics and corresponding corrections.

Whether Soviet technology can keep up with these new ideas that have principally originated in the West is doubtful, considering the present economic climate. So far, the only marketable cesium beam devices available from the Soviet industrial complex are the space-qualified clocks from the Leningrad Radiotechnical Scientific Research Institute. There is no reason why a cesium standard for general laboratory use employing the same cesium beam tube could not be produced by the Soviet Union.

\section{RUBIDIUM ATOMIC CLOCKS}

\section{Summary}

The Soviet Union has achieved superior rubidium performance than that seen in the West. The Soviet approach to this technology is very sophisticated and should allow Soviet researchers to contiriue to advance the state of the art in rubidium. With the link between the basic technologies of rubidium and the Soviet optical pumping objectives in cesium, the Soviet Union-with its impressive group of experienced, talented scientists and engineers-could utilize this knowledge to realize clock and frequency standard technology beyond that which might be expected from the West. 


\section{Discussion of Soviet Work}

The Soviet Union has expended considerably more effort on the research and the industrialization of rubidium vapor frequency standards than on cesium standards.

Most worldwide progress in the field of atomic standards occurred during the 1960s, 1970s, and 1980s. For the most part, the Soviet literature on atomic standards has been concentrated in the last two decades-with little hardware being executed prior to 1970. However, there are a number of papers which reference work associated with rubidium masers and optically pumped rubidium vapor frequency standards that date back to the late 1960s and early 1970s. These papers deal with the underlying physics and physical chemistry, but they do not address experimental instruments.

As previously stated for the other atomic standards, Soviet researchers are well read on Western technology. In the field of rubidium vapor frequency standards, there is a correlation between the Soviet technical timeline and the availability of detailed literature on rubidium technology. In this case, a reflection of the general work of Vanier and his coworkers at Laval University in Canada is noticed (including his published reports on rubidium maser technology).

A paper by Bespalova and Pikhtelev (1983) reports on a technique of filling cells on a vacuum/gas fill system while monitoring frequency. This was some 10 years after E. Jechart (then of Efratom $\mathrm{GmbH}$ ) utilized this same technology in his Munich laboratory.

This does not imply any discredit to the quality of the Soviet efforts. In fact, the work reported is first-rate and it provides the Soviet Union with a distinct advantage in its visible commitment to make a new family of optically pumped atomic standards. M. B. Gornyy, a leader in the development of new optical standards who received his doctorate in Leningrad in 1981, prepared a dissertation on rubidium physics that focused on the use of buffer gases and special cell configurations. It is from such depth that Soviet researchers have and will continue to make excellent progress in the field of optical pumping. 


\section{Projections for the Future}

The Soviet Union does own some state-of-the-art Western rubidium standards. An article by Dubkov et al. (1984) reports on measurements of HewlettPackard's HP-5065A and Rhode \& Schwartz's Efratom XSRM rubidium standards and compares the performance of these Western devices with Soviet Ch1-69 rubidium performance. The results of these comparisons indicate superior performance of the Soviet Ch1-69. The article states that "...a number of [Ch1-69 standards] have been used for several years in organizations of the State Standards Institute of the [Soviet] Academy of Sciences and other places." It also states that "...the design of industrial rubidium..." has made it "...possible to improve the technology of producing electronic components... [thereby] increasing their reliability." As with the hydrogen maser, there appeared to be large quantities of rubidium instruments, which was not found to be the case with cesium instruments.

The following papers further confirm the production of rubidium vapor frequency standards:

- A paper by Gornyy (1987) states that batches of cells "...were developed more than 25 years ago and have long been in mass production."

- A Soviet data sheet offered a commercial rubidium device (Ch1-48) for general sale in 1982.

- A paper by Dubkov et al. (1984) announces the Ch1-69, an industrial standard with 14 units measured.

- A paper by Kaminskiy and Nikulin (1984) describes the use of rubidium instruments for metrological support in the field.

Recently, the Ch1-74 was revealed as a versatile mobile/transportable unit for calibrating frequency standards at $100 \mathrm{kHz}, 1 \mathrm{MHz}$, and $5 \mathrm{MHz}$ (Kalaytanov and Boriskin, 1984). The fact that it operates on either 220 or $115 \mathrm{VAC}, 50-60 \mathrm{~Hz}$ (and $400 \mathrm{~Hz}$ ) may be coincidental to its striking resemblance to the HP-5065A 
rubidium (to be fair, the pictures we have seen are quite vague). It is surprising that more duplication of Western technology (so-called Chinese copies) has not been observed, considering that Western cesium and rubidium instruments have been confirmed to exist in Soviet laboratories for many years. Many nations who either do not belong to or support the strict rules of COCOM now make it easier for the Soviet Union to obtain these standards.

Work undoubtedly will continue in rubidium since it is a very useful, lowcost, compact device. When the highest performance is not required, it fills the need very nicely. If better short-term performance can be obtained, rubidium will become even more important because it could be used as a flywheel for advanced standards such as trapped ion devices.

It appears that the major reason for the early Western advantage in precise time and time interval technology was related to the "soft" Soviet industrial base for manufacturing the many components necessary for a complex atomic standard. Should industrialized countries (such as Japan and those in Central Europe) sell components and technology to the Soviet Union, this advantage could shrink overnight. Recent Soviet visitors to the United States indicate that rubidium instrument production is currently 400 to 500 units per year.

\section{E. HYDROGEN MASER FREQUENCY STANDARDS}

\section{Introduction}

The atomic hydrogen maser was invented in 1959 by N. F. Ramsey and D. Kleppner at Harvard University. The term maser is an acronym for microwave amplification by stimulated emission of radiation, however, in the hydrogen maser, the amplifier is fed back to itself and made to oscillate. Figure III.4 is a conceptual diagram of: (a) the hydrogen maser, and (b) the energy levels of atomic hydrogen.

Atomic hydrogen in the electronic ground state can exist in two basic energy levels, designated by the symbols $F=1$ and $F=0$, depending on the alignment of the spins of the proton and electron and their associated magnetic dipole moments. Two stable configurations of the coupled precessing system can occur: 
(i) when the spins are aligned ( $F=1)$, and (ii) when they are opposed $(F=C)$. When an external magnetic field is applied, an atom in the $F=1$ state can exist in one of the three configurations shown (labeled $m_{F}=+1,0$, and -1 ), which represent the three possible orientations of the rotating magnetic dipole in an external magnetic field. An atom in the $F=0$ state can exist only in the $m_{F}=0$ state. The energy of atoms in each of these four states depends on the external field, as shown in Figure III.4. At room temperature, atoms are distributed nearly evenly among the four states.

(a)

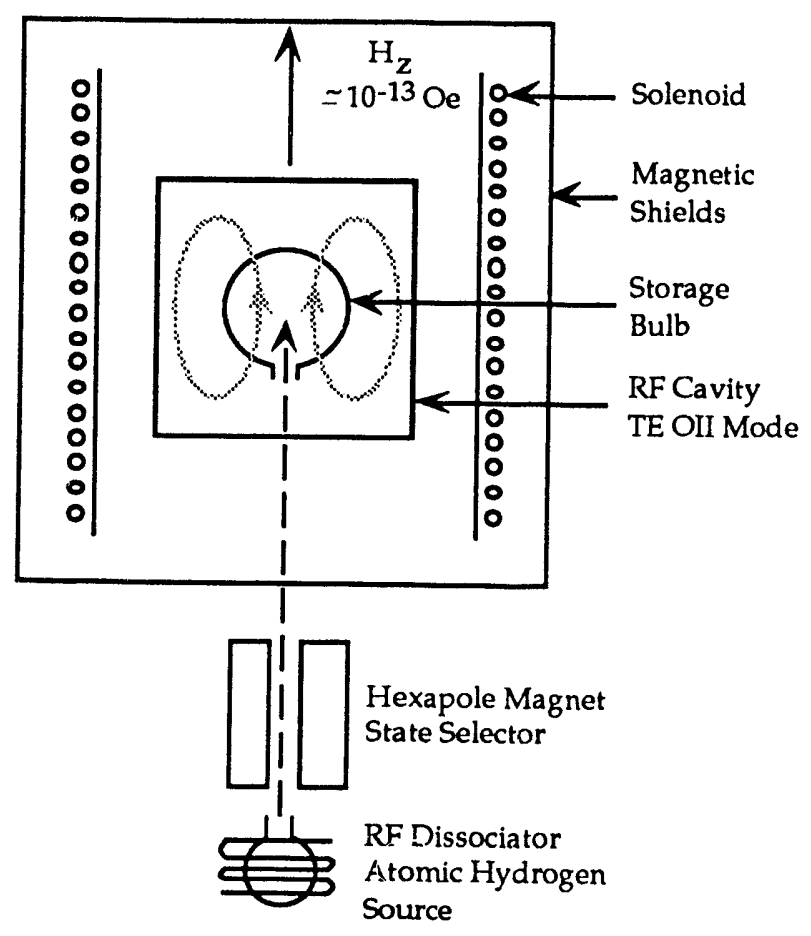

(b)

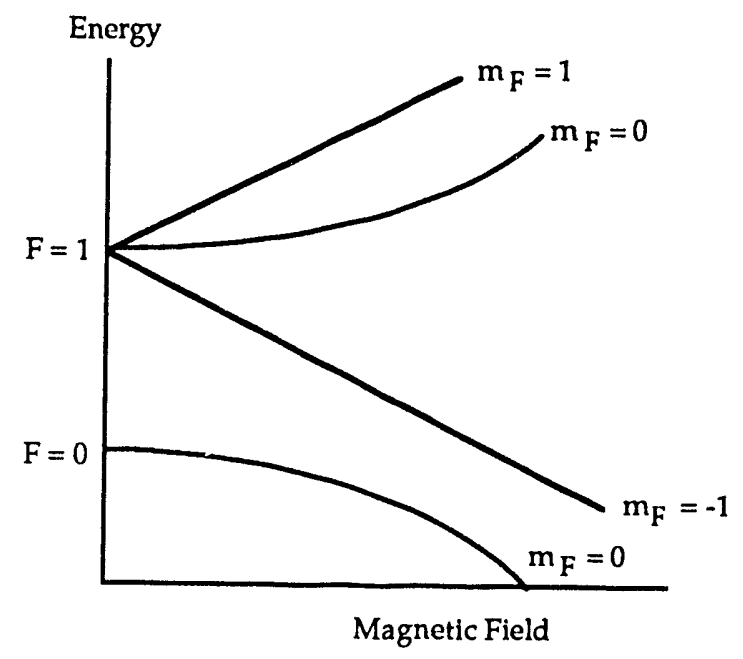

\section{Figure III.4}

(a) Schematic of a Hydrogen Maser and (b) Energy Level Diagram of Atomic Hydrogen

To oscillate, the maser must have a power source. This power is obtained from a constant stream of atoms in an upper energy state entering a region where they encounter a field rotating at the same rate as their precession. These atoms are stimulated to radiate their energy and fall to their ground state, each atom delivering its quantum of electromagnetic energy coherently to the stimu- 
lating field. Since the energy of atoms in the $F=1, m_{F}=0$ state, and the $F=0$, $\mathrm{m}_{\mathrm{F}}=0$ state varies as the square of the magnetic field at low fields, the transition frequency, $\mathrm{f}_{0}$, increases with magnetic field by an amount $\Delta \mathrm{f}_{0}=2751 \mathrm{H}^{2}(\mathrm{H}$ is in oersteds). The value of $f_{0}$ at zero field (and at rest with the observer, or at zero temperature) is $1,420,405,751.7682 \pm .0014 \mathrm{~Hz}$.

Ramsey and Kleppner's basic discovery was that hydrogen atoms, traveling at room temperature velocities of several kilometers per second, could withstand tens of thousands of collisions with specially coated surfaces. This allows storage for a period of 1 second without significant decorrelation of the phase of the signal generated by the atoms. The quality and nature of these surfaces are crucial to the success of the hydrogen masers, and are the principal limitation to the accuracy of the hydrogen maser.

The vessel in which the atoms are stored is located inside a cavity resonator that serves to apply the stimulating RF magnetic field, and as an antenna to receive the atoms' energy. This resonator must have a sufficiently high quality factor, or $Q$, so that a sufficiently large stimulating field can be generated to stimulate the incoming atoms and sustain the oscillation.

There is a limit to the power level at which the maser will oscillate that Lepends on the interatomic collision rate within the storage vessel. Moving atoms can interact in such a way that their phase coherence with the RF field is disturbed or even completely lost. There are other collisional disturbances that cause a systematic frequency shift in the oscillation that is proportional to the loss of the coherence. However, if the atoms in the bulb are all in the $\mathrm{m}_{\mathrm{F}}=0$ states, or accompanied by atoms distributed evenly among the $m_{F}= \pm 1$ states, the resulting frequency shifts can be effectively eliminated. When there are imbalances in that distribution, magnetic gradients in the storage vessel cause frequency shifts that seriously affect the accuracy of the maser.

The process for obtaining atoms in the desired state involves atomic beam techniques. Molecular hydrogen from a pressure vessel (or from various metallic hydrides) is supplied at a controlled pressure of about 0.1 torr to a glass or quartz vessel where an RF field is applied to make a plasma discharge and cause dissociation. Hydrogen atoms emerge from a collimated hole in the glass vessel 
and are led as a beam down the axis of a hexapole (sometimes quadrupole) magnet. As can be seen in Figure III.4, atoms in the $F=1, \mathrm{~m}_{F}=+1$, and $\mathrm{m}_{F}=0$ states require energy to go to high magnetic fields and will fall towards low fields, while those in the other two lower states will fall towards high fields. The very strong radial gradient of magnetic field in a multipole magnet causes atoms in the upper states to move radially inward and be focused into the storage bulb. Normally, equal numbers of atoms in both upper levels enter the bulb. Using additional magnetic systems, it is possible either to isolate atoms only in the desired state or to distribute unwanted atoms evenly among the $\mathrm{F}=0, \mathrm{~m}_{\mathrm{F}}= \pm 1$ states and avoid frequency shifts from interatomic collisions.

\section{a. Causes of Frequency Inaccuracy and Instability in the Hydrogen Maser}

To understand and assess Soviet progress, it is appropriate to review the frequency-governing properties of hydrogen masers, their electronics systems, and the requirements for their raliable operation.

\section{(1) Storage-Vessel Coatings}

Ideally, hydrogen masers should have a storage volume with a surface that minimizes the rate of collision and a low energy of surface interaction with the colliding hydrogen atoms. The energy of interaction between atomic hydrogen and fluorocarbons is small, and to date, various fluoroplastics appear to be the best surface available at room temperature.

The principal limitation of the accuracy and reproducibility of the hydrogen maser frequency standard is the stability and reproducibility of the wall coatings. Surfaces to which hydrogen atoms can stick cause recombination. Changes in the energy of hydrogen interaction due to various surface contaminants cause changes in the way the average phase of the precessing atom is disturbed during a collision. The average phase shift, multiplied by the rate of collisions, catses a frequency shift in the ensemble of atoms interacting with the microwave field. The difference of this shift from maser to maser and, in some instances its variability with time, is the principal disadvantage of the hydrogen maser as an absolute frequency standard. 
Since 1962, teflon has been used for wall coatings in the West almost exclusively in two forms: (i) polytetrafluoroethlyene (PTFE), a long chain polymer; and (ii) fluorinated ethylene-propylene (FEP), a multi-branched co-polymer. PTFE has a linear (and usually heavier) molecular structure and requires a higher temperature to fuse. When hot, FEP remains amorphous and flows fairly evenly, like a high-viscosity varnish. Because of its structure, FEP can harbor more groups of non-fluorine atoms at the ends of its molecular branches than PTFE. Since 1962, there has been little Western effort to find better coating materials, although processes for applying teflon may have improved. There is evidence that the Soviet Union has a superior coating material, which is designated as "polymer F-10."

\section{(2) The Cavity Resonator}

The cavity resonator must have a fairly high $Q$ (usually about 40,000 ) in order to sustain a sufficiently high RF magnetic field to stimulate the atoms to radiate. This is necessary since the power available from the atoms is quite low $\left(\sim 10^{-12}\right.$ watts) because of limitations in the allowable density of atoms. As in any pair-coupled resonators whose resonance frequencies are nearly equal, there is a frequency "pulling" effect for the overall resonance that is proportional to the $Q$ of the respective resonators times their frequency difference. In the case of the hydrogen atom, its resonance quality factor, or line $Q\left(=Q_{1}\right)$ is given by the ratio of the frequency of oscillation to the resonance line width, $f / \Delta f=\pi f T_{2 t}$, where $T_{2 t}$ is the overall unperturbed storage time.

Since the line $Q$ is normally about $2 \times 10^{9}$, and the required resonator $Q$ is about 40,000 we have that

$$
\Delta \mathrm{f}_{\text {out }}=\Delta \mathrm{f}_{\text {cavity }} \mathrm{Q}_{\mathrm{c}} / \mathrm{Q}_{\mathrm{l}}=\Delta \mathrm{f}_{\text {cavity }} \times 2 \times 10^{-5}
$$

If we expect 1 part in 10-15 frequency stability, we must keep the cavity resonator's frequency within $0.07 \mathrm{~Hz}$. This requires dimensional stability in the tenths of microns. To date, the most stable materials in use are glass-ceramics known under trade names such as CERVIT (Owens-Illinois) and ZERODUR (HeraeusSchott). Similar glass-ceramic materiais are now used in Soviet masers. 


\section{(3) Shielding and Control of Magnetic Fields}

While it is desirable to operate the maser at a low magnetic field in order to reduce its sensitivity to changes in magnetic field, the problem of magnetic gradients becomes more severe at low fields. Typically, a field of about 0.5 milligauss with gradients of a few tenths of a microgauss is applied in a 7-inch diameter spherical volume. The internal field must be kept constant within 0.5 microgauss to maintain frequency stability of 1 part in $10^{-15}$. Even in the presence of ambient field variations of only a few hundredths of a gauss (the Earth's field is about 0.5 gauss), very good multiple-layer magnetic shields are required. A number of good materials such as Moly-permendur and Hypernom are available in the West. Similar materials are becoming available in the Soviet Union for use in atomic clocks.

\section{(4) Electronic Systems}

The electronic system for obtaining a useful signal level at a useful frequency in actively oscillating hydrogen masers involves the use of a quartz-crystal-controlled oscillator to synthesize a signal that is locked in phase to the low-level output of the hydrogen maser. A schematic of a typical system is shown in Figure III.5. Here, the amplified $1,420,405,751-\mathrm{Hz}$ output from the maser is heterodyned to lower frequencies with multiples of the $5 \mathrm{MHz}$ from the crystal oscillator and compared, in a phase detector, with the output from a frequency synthesizer. (The steps of frequency variation in this synthesizer supply the necessary output frequency resolution.) The output from the phase detector is led to the electronic frequency control of the quartz oscillator and controls the phase of the quartz oscillator. The system serves to divide the maser output frequency by an exact ratio. For each cycle of maser output, there is an exact fraction of a cycle of output from the quartz oscillator. A phase-locked system is similar to a gear train in a mechanical clock. This behavior is in contrast to that of a frequency discriminator system, which controls the frequency of the quartz flywheel oscillator. This type of control system is similar to a belt drive where a certain amount of phase slippage is inevitable even though the average frequency is highly controlled. 


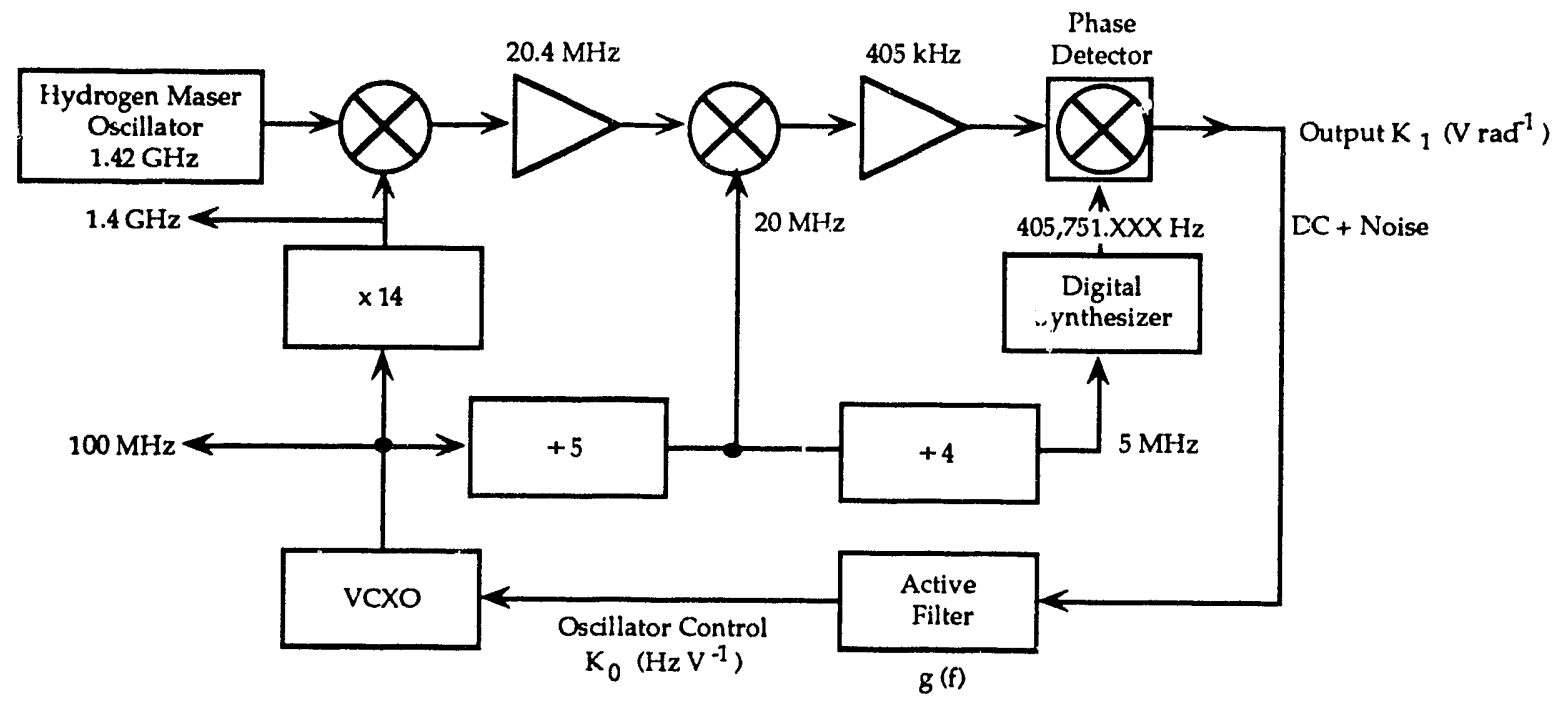

Figure III.5

Electronic System in a Hydrogen Maser

\section{Discussion of Soviet Work}

The idea that molecules and atoms could be used as amplifiers was explored by Townes of the United States, and by Basov and Prokhorov of the Soviet Union, all of whom shared the 1964 Nobel Prize. After the invention of the hydrogen maser in 1959, Soviet activity on these devices began in earnest. An acrount by Basov et al. (1965) describes work which is astonishingly close to that of Ramsey. Their bibliography cites all known work at the time, including data on the six field-operable masers working in the West (mostly in radioastronomical observatories) and comparison data that were made which indicated excellent prospects for fractional frequency stability at $10^{-14}$ or better. At about this time, the Soviet Union decided to use the hydrogen maser both as a frequency standard and as a "frequency store" (that is, a flywheel oscillator to maintain a prescribed or pre-determined frequency). It was only in 1967 that the SI second was defined in terms of the cesium 133 hyperfine structure.

By 1968, B. A. Gaygerov and G. A. Yelkin recognized that the main source of hydrogen maser frequency instability was from cavity mistuning, and they developed a servo method for tuning the maser by observing the amplitude of 
the output signal as the resonance frequency when the cavity is modulated. They stated that this offered an independent method for tuning each maser, in contrast to the usual method where the maser cavity is tuned at a frequency determined by observing the "pulling" of the output frequency (a method that requires a second maser as a stable frequency reference). The cavity modulation process is not as precise a technique, but it is still used today in masers built $n$ both the Soviet Union and in the West. During this time, the Soviet Union was still using the difficult-to-apply PTFE teflon and was suffering large and unpredictable wall shifts. Soviet pregress in this area was lagging that of the W'est by about three years.

Between 1968 and 1972, the Soviet 1 terature reveals little about hydrogen masers. However, in 1972, a substantial effort was reported by Gaygerov and coworkers who described measurements on nine masers (Gaygerov et al., 1972). These masers used titanium getter pumps along with a small ion pump and were said to be capable of normal operation for 5,000 to 10,000 hours (i.e., about one year). Their physical characteristics were almost identical to masers built in the United States. The cavity resonator was made of "crystalline glass," a glassceramic probably similar to the CERVIT used in masers built by the SAO with the usual silvered internal surface. They used a phase-lock receiver controlling a crystal oscillator. The use of titanium getters began at about the same time in the United States for the spaceborne maser flown in the 1976 NASA/SAO relativity test. The Soviet pumps operated at very high magnetic fields, 29 milligauss compared to the 0.5 milligauss levels used in US masers. This indicates that the Soviet Union had difficulty in obtaining good metal for shielding during the early 1970s. These masers required a periodic adjustment and compensation of magnetic field as often as once a day. Soviet researchers reported magnetic disturbances of $4.5 \times 10^{-13}$ in one hour, $3.5 \times 10^{-13}$ in three hours, and $4.6 \times 10^{-13}$ in two hours; they also cited a 300-hour (12.5 days) period for the longest operation, after which the maser was shut down "in normal working state." The reported maser stability, in terms of the Allan standard deviation of measurements, is shown in Figure III.6. The main instability was caused by magnetic field drift, error in the $Q$ compensation setting, and variations in the storage bulb temperature. A six-month preliminary test showed day-to-day frequency drifts within 1 part in $10^{-13}$ and it was concluded, prophetically, that atomic clocks of this type 
"may be recommended as working time reference standards" (Gaygerov et al., 1972).

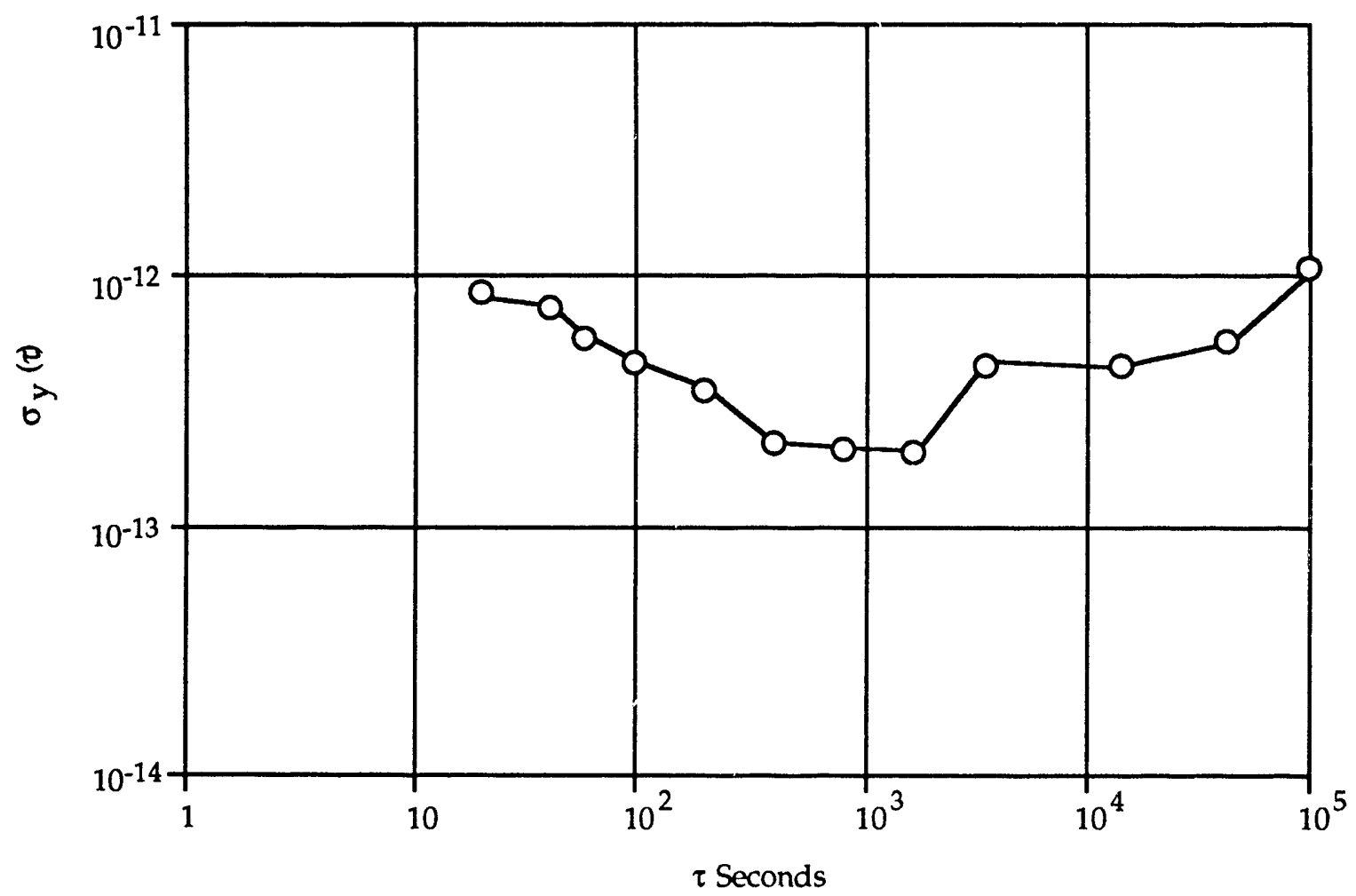

Figure III.6

Frequency Stability of Early Soviet Hydrogen Masers

(Gaygerov et al., 1972)

During this time, Soviet researchers determined the atomic second through the hyperfine interaction of atomic hydrogen in an undisturbed state (Yelkin et al., 1973). This required the frequency of the masers to be not only highly stable, but also accurate. At that time, the Soviet Union was concerned mainly with accuracy, whereas researchers in the West were (and still are) concerned mainly with stability. The issue of atomic time scale accuracy was resolved in 1967 by the use of cesium 133 in the SI system used in most Western countries. 
The motional effect of atoms asymmetrically distributed among the $\mathrm{F}=0$, $m_{F}=+1$, and $m_{F}=-1$ states was the greatest source of error because of their magnetic shielding problem and lack of field homogeneity which required that they operate at high field levels. Soviet researchers adopted a plan for circumventing this problem by alternating the polarity of an axial magnetic field at the exit of the hexapole magnet in order to make a symmetrical distribution of atoms in the unwanted $\mathrm{m}_{\mathrm{F}}=+1$ and -1 sublevels. Though effective in improving accuracy, this system was inefficient and resulted in undesired collisional broadening. A program to measure the wall shift with six bulbs reportedly began in 1970 . The wallshift found is in agreement with Western data for teflon at that time.

A significant Soviet hydrogen maser development is reported by Bogdan et al. (1974). They describe a new Ch1-46 maser that appears to be a totally new approach, and may be the prototype of the Ch1-80 and Ch0-101b devices described in Appendix A. This hydrogen maser model was claimed to be smaller, lighter, lower powered, more reliable, and more convenient, and to have longer life expectancy and better frequency stability and reproducibility. From all appearances, the Ch1-46 is a copy of the SAO VLG-10 masers built in 1970 and has a cavity resonator made of "special glass" (probably a glass-ceramic like OwensIllinois' CERVIT) in the conventional configuration. The reported dynamic magnetic shielding factor of $\mathrm{S}=\Delta \mathrm{H}_{\mathrm{ext}} / \Delta \mathrm{H}_{\mathrm{int}}=1250$ is not good (unless this is a misprint) considering that three shields are used.

In 1976, Zhestkova and coworkers reported on a bulb that is coated with two layers of 4D PTFE and is similar to earlier bulbs used by N. A. Demidov at the Gor'kiy R\&D Instrument Making Institute (Zhestkova et al., 1976). They cited an improved working life and smaller wall shift with this coating. At that time, FEP teflon was a standard coating used in the United States and elsewhere in the West because it was easier to apply than PTFE teflon.

The maser receiver-synthesizer operates by phase locking a $5-\mathrm{MHz}$ quartz crystal oscillator, which can be used in an open-loop configuration to compare the maser to other frequency references. The noise performance of the receiver is poor, compared to 1974 standards of Western technology. The open-loop frequency stability data are better (by a factor of 3) than the closed loop data, compared at $5 \mathrm{MHz}$ (see Figure III.7). The Ch1-46 standard is described as containing 
two hydrogen masers, together with the Ch3-38 electronic frequency meter. By operating a two-maser system, each is able to tune the other using the line $Q$ modulation technique.

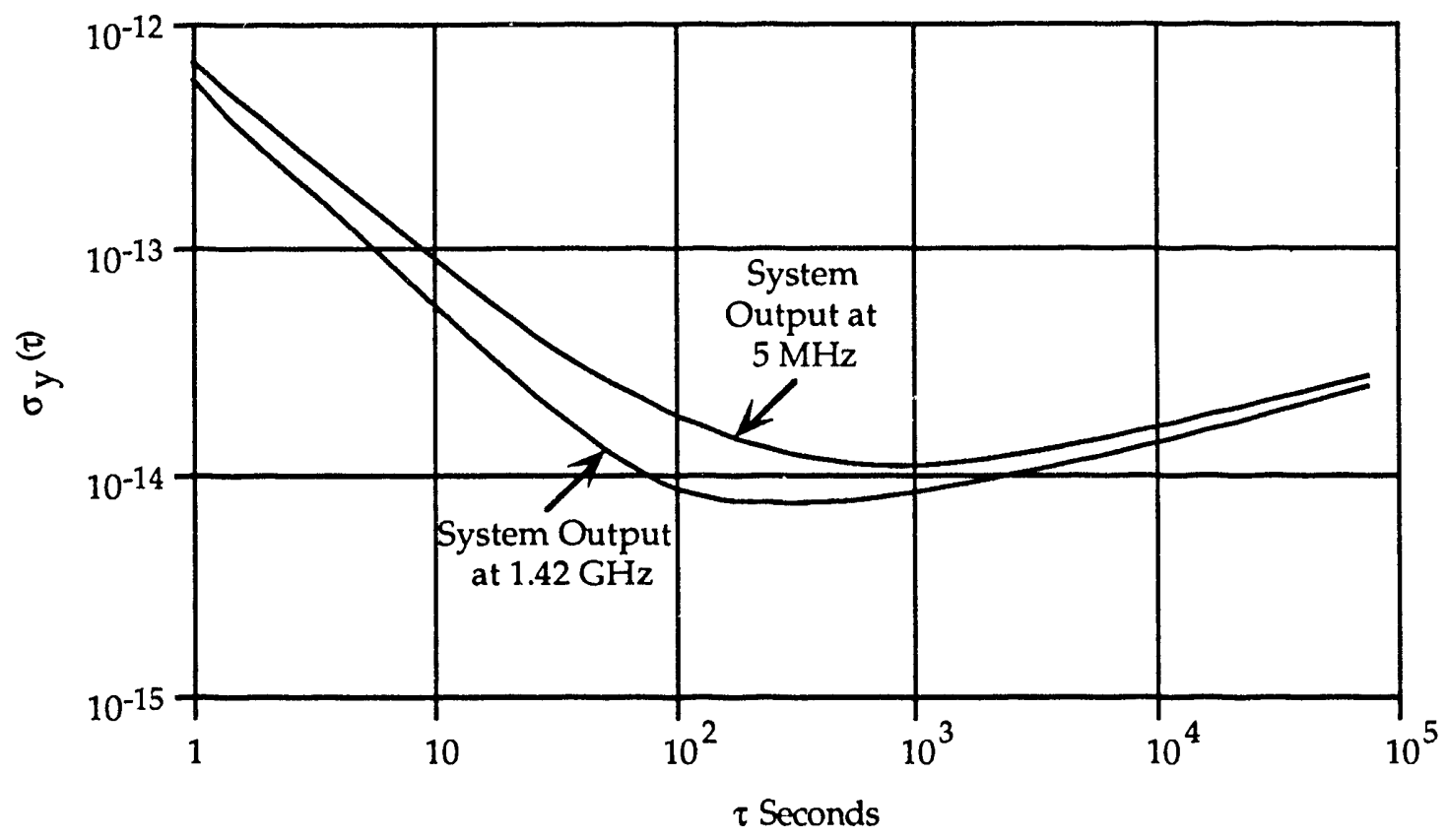

Figure III.7

Frequency Stability of the Ch1-46 Hydrogen Maser

(Bodgan et al., 1974)

The Ch1-46 is cited as being used in laboratories involved in the production and testing of frequency measuring equipment of high stability and accuracy (probably in the production of optically pumped rubidium, and possibly cesium beam working standards). The performance of the Ch1-46 system is very good, and the design of its two hydrogen masers appears to be sound. The life expectancy of these devices appears to be about three years, at a fairly high bearn flux. These devices are probably forerunners of the Ch1-80 masers. This work is comparable to US efforts in the early 1970s.

Korobov et al. (1976) reported on "a new state standard of time and frequency, and methods of realizing its accuracy," citing that the main metrological 
characteristics of atomic clocks improved by a factor of 10 every five years, and that a series of apparatus were being used which operate with an error of $10^{-11}$ to 10-12. The need for a better national standard became evident, and a new state standard was developed by VNIIFTRI with the cooperation other scientists and industrial organizations during the period from 1972 to 1975. The newly produced standards were said to have improved by a factor of 30 , and computerautomated comparisons were made. However, in a significant change of policy, they operated a cesium beam primary standard to reproduce the second of time independently, in accordance with the SI system of units using a highly practical system, where cesium is used to check the frequency of three hydrogen maser standards that have better stability than their cesium device(s) over periods of several months. At a lower level of accuracy, but at a higher level of reliability, they operated an array of three hydrogen maser systems (probably Ch1-80s) and six HP-5061A standards as "custodians" of a working time scale that is computer monitored in order to select the best cesium standard and hydrogen maser to run a working system that provides stable and accurate time and frequency signals. The use of hydrogen masers in the Soviet system no longer appears to be focused on accuracy, but instead on frequency stability over time intervals as long as one year.

An interesting method of controlling a rubidium gas cell frequency standard (HP-5065A) with a hydrogen maser is described by Vishina and Gaygerov (1976). Here, the rubidium standard is phase-locked to the hydrogen maser by controlling the magnetic field of the rubidium gas cell transition region. When the maser quits or the power fails, the rubidium cell standard carries on. It is equipped with a trickle-charged storage battery backup system. This equipment takes advantage of the low drift and good short-term stability of the maser while minimizing the effects of its poor reliability and high power consumption. There appears to be no Western desire to operate such an arrangement of rubidium gas cells and hydrogen masers.

In the early 1970s, the Ch1-44 (an "industrial" standard consisting of two hydrogen masers, probably built at Gor'kiy "Quartz" NPO) was installed in the State Time and Frequency Standard at VNIIFTRI in a "supplementary" capacity. During 1973 and 1974, tests were conducted with the Ch1-44 to improve the error of reproduction of the maser output frequency to a level of 1 part in 10-13. One 
maser was offset $1 \mathrm{~Hz}$ in frequency from the other by raising its magnetic field to 19 milligauss. The other maser was operated at 0.8 milligauss. This method of offsetting the frequencies is crude, and indicates a lack of digital synthesizers.

The masers were tuned in the usual manner by modulating the line $Q$ by varying the beam intensity. The Soviets achieved a $4 \times 10^{-14}$ reproducibility of the relative frequency between the two masers, and in comparisons with the VNIIFTRI-built masers, they obtained a one-month RMS deviation of fractional frequency of $8 \times 10^{-14}$. The VNIIFTRI maser system was also calibrated against the VNIIFTRI cesium standard. A strenuous series of wallshift tests using both FEP and PTFE fluorocarbons were made in an apparent attempt to improve the capability of the hydrogen maser in the role of a primary standard. The Soviets concluded that the measurement error in wallshift was about $1 \times 10^{-12}$ and that this was the most fundamental error in determining the undisturbed hydrogen frequency. The Soviet data on wallshifts are consistent with results obtained elsewhere at that time.

After correction for temperature (second-order Doppler) and magnetic effects, it was concluded that the undisturbed hyperfine frequency for atomic hydrogen was $1,420,405,751.7682 \pm 0.0014 \mathrm{~Hz}$. This 1977 result may be a clear improvement over the value of $1,420,405,751.768 \pm 0.002 \mathrm{~Hz}$ that was obtained by Hellwig and coworkers in $1970,{ }^{3}$ which strangeiy enough, was not 'acluded in the references cited by Zhestkova et al. (1976), in which these data were reported.

By 1976, the role of the hydrogen maser in Soviet timekeeping and frequency standardization further evolved in the direction of being a highly stable flywheel (Yelkin and Purtov, 1976). However, hydrogen maser wall coating work progressed and studies of wall shift continued, and signifirant progress was made. New work reported by Yelkin et al. (1977) references very completely the work done in the West, including techniques involving storage volumes of variable volume that originated at Harvard University in 1968. Their results with a new fluoroplastic, described as "polymer F-10" applied in the usual quartz spherical

3 H. Hellwig, R. F. C. Vessot, M. W. Levine, P. W. Zitzewitz, H. E. Peters, D. W. Allan, and D. J. Glaze, "Measurement of the Unperturbed Hydrogen Hyperfine Resonance Frequency," IEEE Trans. Instr. Meas., IM-19, (1970), 200-209. 
storage volumes, are quite remarkable. The melting temperature for polymer F-10 is considerably lower than the $360^{\circ} \mathrm{C}$ temperature used by SAO for curing FEP-120 teflon. The Soviet F-10 material is a different polymer that has a lower melting point and better capability to form a smooth finish, therefore, it has a lower collision rate than FEP-120. Their results are impressive, even at this date, some 13 years later. They obtain about five times lower values of wall collision relaxation and wall frequency shift than is typical of Western hydrogen masers. Their wall shift for a 7 -inch diameter sphere is -4.7 parts in $10^{-12}$, and even lower numbers are mentioned in the Ch1-75 maser prospectus. The lower values permit greater reproducibility in the output frequency and better prospects for frequency accuracy. Soviet wall relaxation is significantly lower than that of the West. They cite a line $Q$ of $2 \times 10^{9}$ for a 4-inch diameter sphere, and $1.1 \times 10^{10}$ for the usuai 7 -inch diameter sphere. Thus far, the best results obtained in the West have been line Qs of about $6 \times 10^{9}$ for a 7-inch sphere. Clearly, much can be learned from Soviet research on wall coatings, which is pivotal to the maser technique.

Frequency shifts from interatomic collisional processes were reported by Yelkin and Zhestkova (1979) following the 1975 work in the United States by S. B. Crampton and H. T. M. Wang. These sources of frequency instability were not a serious problem in Western hydrogen masers, probably because of the availability in the West of good magnetic shields.

One of the first problems the United States encountered in maintaining hydrogen maser frequency stability was in automatically maintaining the tuning of the maser's cavity, and, like many US technological developments at that time, this was repeated in the Soviet literature with little credit to US innovators. Three methods are presently in use: (i) tuning by setting the resonator's frequency so that no output frequency shift is observed when the oscillation line $Q$ is modulated, (ii) tuning by setting the resonator's frequency where the output signal is strongest, and (iii) tuning by measuring the transmission of signals introduced into the resonator at frequencies near the inflection points of the cavity resonance. Methods (i) and (ii) are accompanied by modulation of the maser signal. Method (iii) does not disturb the oscillation, but requires an external measuring circuit of exquisite stability. 
A review article by Gaygerov (1972) discusses these tuning processes and describes the development of masers with a vacuum system using "titanium absorbers as the basic means for evacuating hydrogen." In 1976, a successful test of the general theory of relativity was conducted in the United States with a spaceborne hydrogen maser that operated prior to launch for one year using a metallic getter pump made of activated zirconium, aluminum, and titanium; however, no reference is made to this well-known technology. Gaygerov describes his magnetic shields as made from "three double-layer magnetic screens made from a permalloy tape $1 \mathrm{~mm}$ thick and $250 \mathrm{~mm}$ wide with welded seams." This indicates a serious lack of suitable shielding material.

Results from operation of Soviet masers in 1977 through 1978 showed that, over a period of 24 hours, one-sigma variations of frequency of $5 \times 10^{-14}$ could be expected. No systematic variations of the frequency greater than $1 \times 10^{-13}$ were observed. Occasional unexplainable variations as large as $2 \times 10^{-13}$ were seen. The probable causes cited for these variations were: (i) changes in magnetic gradients in the storage vessel from variations of external magnetic fields, or (ii) thermal currents in the resonator shields or vacuum belljar. Judging from the metal available for shielding and the method of manufacture (there is no mention of annealing, which is essential for good performance), these are probably reasonable performance expectations. The problem of thermally induced electric currents has long been recognized in US work on microwave atomic standards; the fact that Gaygerov mentions this problem as late as 1979 is surprising. He concludes that there is a need for "a radical means for raising the hydrogen maser stability [that] consists of forming an atom beam which contains only atoms in the $\mathrm{F}=1, \mathrm{~m}_{\mathrm{F}}=0$ state." However, he makes no mention of the work conducted 10 years earlier by Lacey and Vessot in the United States, and by Audoin in France, where such methods were being developed, although these methods have only recently been operated in practical systems. This type of state selection has been incorporated in hydrogen masers built by SAO since 1979.

By operating with cavity autotuning and periodic inversion of the $F=1$ states to make a symmetrical distribution of atoms in the $\mathrm{m}_{\mathrm{F}}=+1$ and -1 states, Gaygerov reports a one-sigma deviation in $\Delta \mathrm{f} / \mathrm{f}$ of $2 \times 10^{-14}$ over a 24-hour period. 
While the designs of the Soviet systems certainly were more than adequate, both the available metal for shields and the cleanliness of their vacuum systems were less than desirable. Despite such shortcomings at this stage, it is clear that there was large-scale production of hydrogen masers in the Soviet Union, and although the Soviets were lagging the United States in a number of areas, they knew what th.ey needed to do. The Soviet technology is no longer lagging that of the United States. The Soviet hydrogen maser production rate is substantially higher (by approximately a factor of 5) than that of the United States.

An article by Gaygerov et al. (1982) gives a penetrating view of Soviet progress with 10 Ch1-46 hydrogen masers, eight of which were autotuned by the Q-modulation technique, and two of which were running with periodic frequency corrections done manually. No masers were switched off. The frequency stability of the $5-\mathrm{MHz}$ signals was evaluated and the error in reproducing a specific frequency was measured over a one-year period. The free-running maser system stability, measured at $5 \mathrm{MHz}$, is shown in Figure III.8. In the autotuned systems, there was a deterioration in the frequency stability due to the amplitudeto-phase variation in the phaselock receivers. Two of the 10 automated masers varied by 2 to $3 \times 10^{-13}$ per month and "were several times modified," and were not included in the statistical evaluation of the ensemble. Data for the six remaining autotuned masers were shown over a one-year period. Two of these masers had rather steady variations of frequency of 2 to $3 \times 10^{-14}$ per month (one upward, the other downward in frequency). The authors checked the cavity temperature and average magnetic field in bulb "not less than once per month." There was no evidence of any frequency variation attributable to the average field or to temperature-induced wall shifts. They checked for magnetic field inhomogeneity shifts in two ways: (i) by inverting the population in the $F=1$, $m_{F}=+1$, and $m_{F}=-1$ levels; and (ii) by periodically varying the average DC field at the exit of the magnet, as described earlier. While this helped to reduce the frequency variation, it was concluded that the systematic long-term behavior was almost certainly due to "deficient technology in the coating of the bulb or contamination of the surface." 


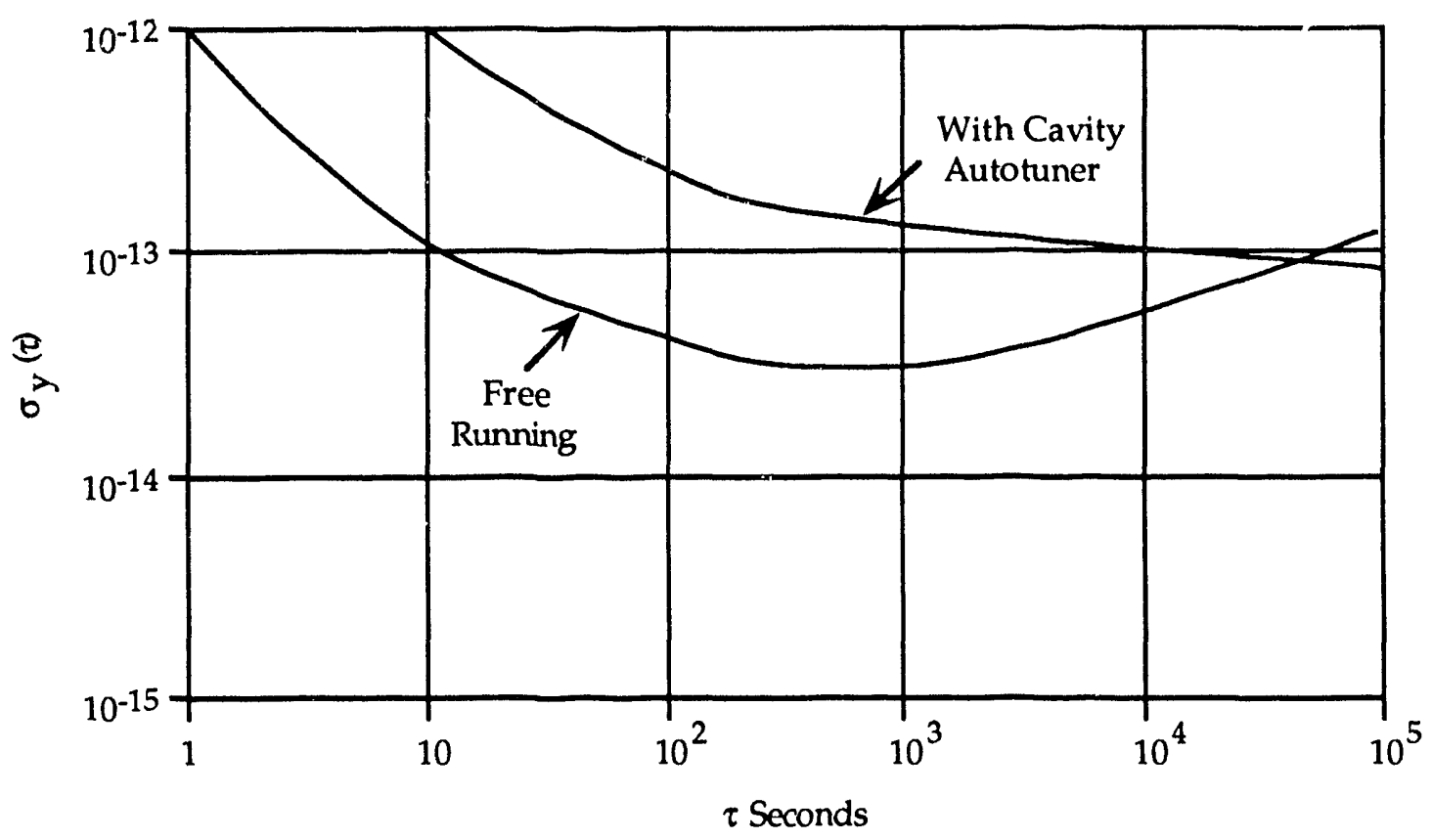

Figure III.8

Frequency Stability of the Ch1-46 Hydrogen Maser at VNIIFTRI

(Gaygerov et al., 1982)

These were carefully conducted tests that revealed the major problems in hydrogen maser technology. Frequency drifts of a few parts in $10^{-15}$ per day or, about 1 part in 10-13 per month, are not uncommon in masers built in the West. When compared against the Bureau International de L'Heure (BIH), some US hydrogen masers have shown wall collision frequency variations of less than 2 parts in $10^{-13}$ per year, and some have no measurable shift. Generally when such variations occur, the trend is to go to lower frequency with a measurable reduction in the oscillator's line $Q$.

It was concluded that, in the ensemble of masers operated continuously with automatic cavity tuning, the error in frequency reproduction, defined as the standard deviation of mean monthly values from the mean for the year, is 2 to $4 \times 10^{-14}$. These masers were used as reference sources in the Soviet State Standard of Time and Frequency, and at that time, were at the current state of the art.

A complete review of hydrogen maser progress was given by Gaygerov et al. (1983) who, for the first time in a Soviet journal, referenced articles from the Pre- 
cise Time and Time Interval (PTTI) Applications and Planning Meeting. The 1976 SAO/NASA GP-A experimental test of the gravitational redshift test with a spaceborne maser is mentioned, but the stated size, weight, and power is grossly in error (440-660 pounds vs. 95 pounds, and 200 watts vs. 70 watts). Efforts to reduce the size and weight of hydrogen masers are discussed in detail. The survey concludes that "it can be expected that in the very near future hydrogen frequency standards of both stationary and transpcrtable type(s) will be developed with frequency instability less than $1 \times 10^{-14}$ per day" and that for longer time intervals, the instability will depend on the variability of the wall shift.

The passive hydrogen maser, which works as an extremely narrowband amplifier that is operated as a frequency discriminator to control a crystal oscillator, was the subject of considerable work in the late 1980s by Belyayev et al. (1987) from the Gor'kiy "Quartz" NPO. The work described closely follows the 1976 and 1979 electronic systems of the US National Bureau of Standards, and a smal'-size cavity resonator designed and patented by H. E. Peters is acknowledged in its references. The properties of these resonators were calculated by Belyayev and Savin (1987). Much effort was expended to obtain a smaller, more transportable device for synchronizing distant stations. The stability data are shown in Figure III.9 where the smaller version of the experimental maser has a reported stability of $\sigma_{\mathrm{y}}(\tau)=3 \times 10^{-12}\left(10 \mathrm{sec}<\tau<10^{3} \mathrm{sec}\right)$, and the larger system has a reported stability of $\sigma_{\mathrm{y}}(\tau)=1 \times 10^{-12}\left(10 \mathrm{sec}<\tau<10^{3} \mathrm{sec}\right)$.

These comparisons reportedly were made using the Ch1-75 maser as a reference. This device is described in a prospectus in Appendix $A$; it has a reported stability of $5 \times 10^{-13}$ in 1 second and $5 \times 10^{-15}$ in 24 hours.

Work on the passive hydrogen maser continued in 1987. Quantity production of the Ch1-76 small-size, passive hydrogen frequency and time standard ensued. A prospectus on this device is included in Appendix A. This device uses a double-frequency correction servo loop for both the quartz oscillator and the cavity tuning, a sophisticated system that originated in Switzerland using only one modulation frequency to control both the cavity and the quartz oscillator to the atomic resonance. Its short-term performance is guaranteed to be better than $3 \times 10^{-13}$ for 1 second, with a systematic error of less than $\pm 1.5 \times 10^{-12}$, and a reproducibility of $\pm 3 \times 10^{-13}$ (see Figure III.9). 


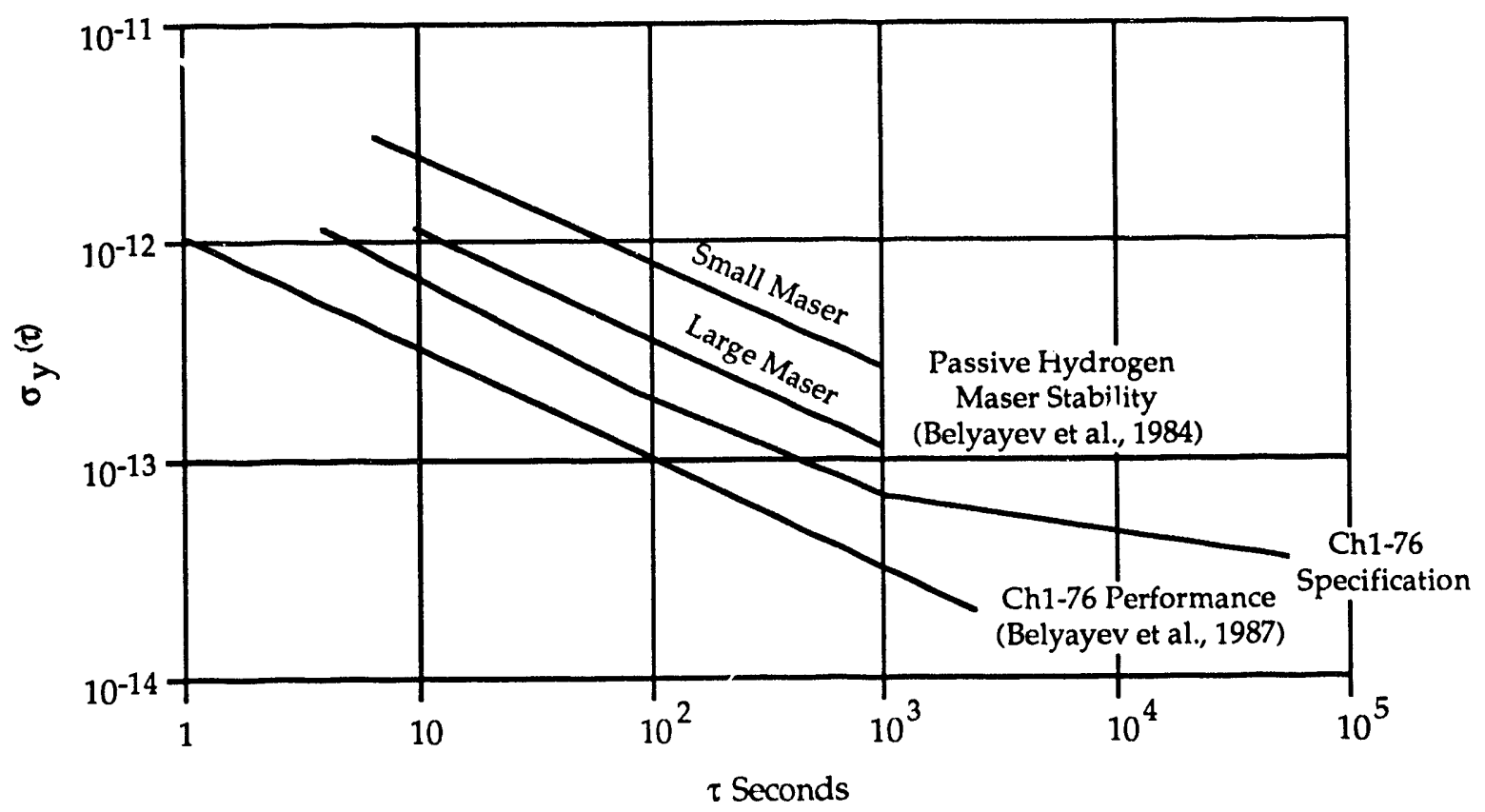

Figure III.9

Passive Hydrogen Maser Performance (1984-1987)

This is better stability thas has been achieved with the NBS (now the National Institute of Standards and Technology [NIST]) prototype passive maser, which is probably due (at least in part) to the superior Soviet F-10 teflon wallcoating material. The mechanical design of the Ch1-76 is rugged and allows efficient and cost-effective manufacture of the critical cavity resonator storage vessel assembly. The magnetic shielding is good and it uses two types of magnetic alloys. Hydrogen is supplied by warming a small quantity of $\mathrm{LaNi}_{5} \mathrm{H}_{\mathbf{x}}$ and it is flow-controlled and purified by permeation through a nickel tube heated by its resistance. State selection is done by a 4-pole magnet $75-\mathrm{mm}$ long with a $1.6-\mathrm{mm}$ bore diameter exactly following the innovations made by H. E. Peters some years ago. This standard is at the state of the art and appears to be highly competitive with, if not ahead of, any US product.

Some idea of the behavior of the Soviet equipment can be obtained in a report from the State Time and Frequency Service (STFS) of the USSR dated March 1989 to the Consultative Committee for the Definition of the Second (CCDS). The State Primary Time and Frequency Standard (SPTFS) is maintained 
at the main metrological center of the State Time and Frequency Service located in VNIIFTRI near Moscow, and a set of five secondary standards are located in Uzhgorod, Khar'kov, Novosibirsk, Irkutsk, and Khabarovsk. Only VNIIFTRI uses cesium beam primary standards; the other stations use hydrogen masers which are synchronized by various techniques.

The CCDS report indicates that the STFS has more than 15 hydrogen masers of the stationary, "commercial" Ch1-70 type that operate as pairs, with automatic cavity tuning servo-systems. During normal operations, roughly 10 of these masers are on-line, and of these, only the three to five with the best long-term stability are used in the Soviet atomic time scale TA(SU) and the UTC(SU) time scale. Both of these time scales are described as "paper clocks" because the STFS does not generate a physical signal corresponding to the UTC(SU) time scale. The CCDS report states that these masers have been operating during the past several years, but there have not been any serious physical investigations or modifications to them. Their stability is described as 2 to $3 \times 10^{-14}$ from $100 \mathrm{sec}-$ onds to several days. The report also states that "unfortunately the reliability of these instruments is not high enough, only some masers work more than a year, the usual time for continuous operation for time-scale generation does not exceed six months."

Typical substations use four Ch1-70 hydrogen masers that have stabilities comparable to those of the SPTFS masers. The performance of these masers reportedly depends "primarily on the environmental conditions and the qualification of the operators."

Among the methods for time-scale comparison, Soviet researchers report use of a portable, compact hydrogen maser, called "Sapphire," which is currently in commercial production. Sapphire is an active maser using a small, dielectrically loaded cavity. Its cavity has a $Q$ of 50,000 with a storage volume of $790 \mathrm{~cm}^{3}$ (Rusin et al., 1985). Because of the large temperature coefficient of the dielectric constant of Sapphire, it is necessary to use an automatic cavity stabilization system (Gaygerov and Yelkin, 1968). With this system, the thermal stabilization problem is controlled to provide a level of stability of $3 \times 10^{-14}$ from 100 seconds to one day. The dimensions of the entire clock system are $12 \times 18 \times 22$ inches, its weight is 136 pounds, and it operates for five hours on its internal 40-watt power 
supply. Apparently, this device is brought to Cuba once a year to synchronize six hydrogen masers at a site near Havana.

The March 1989 report to the CCDS also describes a portable clock, called "Sphere," $w$.ch is another active maser using a spherical full-size cavity made of quartz, and therefore has a lower temperature dependence. It also states that the cavity resonance $Q$ is 50,000 and the maser line $Q$ is 1.5 to $2.23 \times 10^{9}$ and that $\sigma(\tau)$ is best at 1,000 seconds. This clock is 16 inches in diameter, 29 inches high, and weighs 143 pounds with batteries (110 pounds without). It has no automatic tuning servo electronics, however, provision has been made for such tuning by including a second coupling loop in the cavity. Figures III.10 and III.11 show the configuration of the Sapphire and Sphere portable masers. While most of the previous work has been done at Gor'kiy "Quartz," the Sphere and Sapphire hydrogen masers have been developed at VNIIFTRI.

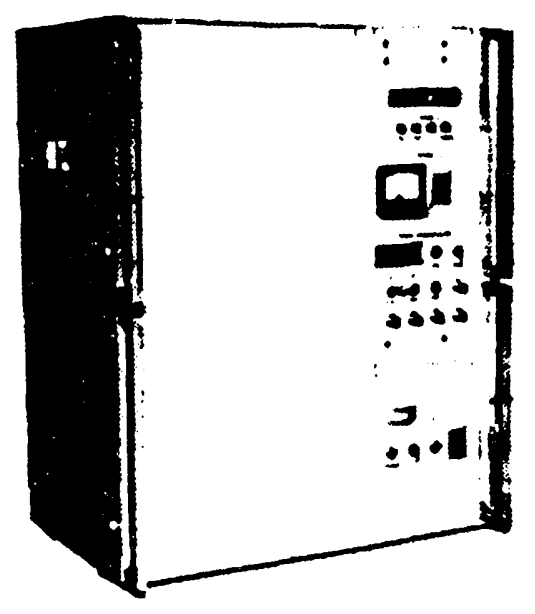

Figure III.10

Portable Clock-Sapphire 


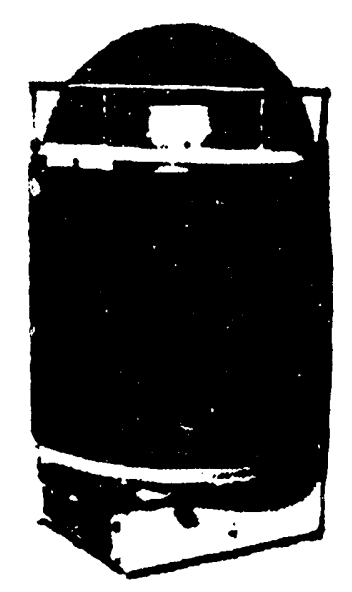

Figure III.11

Portable Clock-Sphere

While these masers represent no major technological breakthroughs, they represent a suhstantial effort, and they make use of the high-production capacity for hydrogen masers available in the Soviet Union. The Soviet development of portable hydrogen masers, in preference to the development of cesium or rubidium devices, indicates a high level of confidence in their hydrogen maser technology in comparison to that of other possible devices.

It is clear that, upon entry into the production of atomic frequency stan.dards, the Soviet Union made a major commitment to produce hydrogen masers. This may have been a legacy of the Basov-Prokhorov 1964 Nobel Prize, which placed the Soviet Union at the forefront of maser research. The technology for hydrogen maser development may, at that time, have appeared to be simpler than the more mature cesium beam and rubidium gas cell standards technology of the West. There is a considerably larger number of individuals involved in hydrogen maser development in the Soviet Union than in the West. These individuals appear to have begun their work at the Lebedev Physics Institute under Basov. Work on hydrogen masers has been conducted at three other centers in 
the Soviet Union-VNIIFTRI (the Soviet bureau of standards), Gor'kiy "Quartz" (the so-called industrial group), and the Leningrad Radiotechnical Scientific Research Institute.

Early Soviet work on hydrogen masers clearly was hampered by a lack of test equipment, electronic subcomponents, and critical rnaterials, particularly magnetic shielding. The Soviet intellectual capability has been first rate from the very start. There is evidence of good theoretical talent and competent, but not spectacular, engineering. Except for a superior wall-coating material, "polymer F-10," there are few Soviet developments that lead those of the United States, even in their most recent products. However, the Soviet production capability is at least 10 times that of the United States; where the United States would build two masers, the Soviet Union would build 10 or more.

Soviet researchers focused their attention on producing both accurate and stable oscillators to operate the clocks in their widely separate timing centers. After the mid 1970s, their quest for hydrogen maser accuracy seemed less important and they developed cesium beam devices to represent the SI definition of the second of time. Today, the hydrogen maser remains the Soviet "workhorse" oscillator. Although Soviet researchers complain about the reliability and operating life of hydrogen masers, they make up for this with redundancy; some 15 to 20 hydrogen masers are reported to be in service at VNIIFTRI, and as many as six are reported to be installed in outlying substations, including Cuba.

The most recent Soviet hydrogen maser developments seen in the Ch1-75, Ch1-80, and Ch-101b systems are state of the art, and some features are ahead of comparable Western devices. The short- and long-term frequency stability of a Soviet Ch1-75 actively oscillating hydrogen maser and a Soviet Ch1-76 passively operated maser was compared with hydrogen masers at the SAO. These two hydrogen maser clocks arrived at Kennedy Airport via Aeroflot, accompanied by Dr. N. A. Demidov and two Soviet engineers. They were then transported to Cambridge, Massachusetts, keeping power on the Ch1-75 maser. The Soviet hydrogen masers were installed in the SAO clock room and compared with three SAO-built hydrogen masers and, by the GPS common-view technique, with the hydrogen masers at the US Naval Observatory. Frequency stability over intervals from 1 second to several days was measured using a time-correlated tech- 
nique to determine the individual statistical behavior of each individual maser. Longer-term comparisons were made to measure the relative frequency drift of the jet of hydrogen masers on hand using the laiest frequency comparison equ pment made available to SAO by ERBTECH Company and NIST. Continuous timekeeping comparisons of the five hydroger masers were made with atomic clocks at the US Naval Observatory, NIST, and the German National Frequency Standards using the GPS spaceborne clocks in the common-view mode of operation in order to remove possible effects of the instability of the space clocks. The performance of the Soviet hydrogen masers is fully comparable to those of the United States (some aspects of the tests are shown in Appendix A).

Soviet portable devices are far ahead of those of the United States. The United States has done little to develop active, non-regenerated $Q$ hydrogen masers of small size and weight. Soviet developments include hydrogen masers for spaceborne applications.

An important Soviet technological advantage is the superior wall-coating material "polymer F-10." This material produces about one-half the wall collision shift, and presumably, one-half the wall relaxation rate of the Dupont FEP-120 teflon used in the United States. Unperturbed confinement of atomic hydrogen is the fundamental basis for the narrow linewidth obtained in the hydrogen maser. There is little or no evidence of Soviet hydrogen maser work at cryogenic temperatures using wall coatings of superfluid ${ }^{4} \mathrm{He}$, although some study is underway on low-temperature hydrogen interactions.

The Gor'kiy "Quartz" NPO manufactures a passive maser, the Ch1-76, that has very good performance based upon the data given in its papers, in the specifications cited in its prospecti, and in recent tests at SAO. While there are some advantages to the small passive maser, the problems of wall coating stability and all the other causes of hydrogen maser instability remain. Although the passive maser technique somewhat reduces the effects of interatomic collision rates by permitting operation at much lower beam flux, the signal-to-noise ratio is necessarily much worse than active masers, and a carefully adjusted modulation and demodulation system is required for frequency control. The Soviet technology for passive masers appears to be comparable to that of the United States and seems to have closely followed the work of Western innovators. 
The most interesting Soviet developments are the small portable clocks Sphere and Sapphire, which are used for time transfer purposes. These are stateof-the-art devices that incorporate all known appropriate hydrogen maser technology into their packages. While these clocks do not represent any technological breakthroughs, except possibly a very effective magnetic field cancelling servo system, they do represent a significantly refined electronics technology. Nearly all of the design features of these clocks originated in Western laboratories. The dielectrically loaded cavity, $\mathrm{H}_{2}$ sorption pumping, metallic hydride hydrogen supply, and many mechanical features such as the stress-free cavity mounting system structure used in the 1976 space probe maser, have found their way into Soviet designs.

\section{Projections for the Future}

The Soviet Union has clearly deployed far more resources, scientists, and manufacturing capabilities to work on hydrogen masers than has the United States and the rest of the West, combined.

Active and passive hydrogen masers will continue to play a strong role in Soviet timekeeping and frequency control. Soviet researchers are working aggressively toward continued performance improvements, particularly those leading to a more accurate realization of the unperturbed hydrogen frequency and to better stability. They have a high production capacity and appear to be gearing up to market these products worldwide.

Opportunities for more US/Soviet cooperative projects in space activities with atomic clocks may occur in the future. During a 1988 visit to Moscow by Dr. R. F. C. Vessot, Dr. N. Kardashev of the Soviet Space Agency suggested that the United States might provide a hydrogen maser system for the Radioastron VLBI spaceborne radiotelescope system. US cooperation has already been approved for this project in the area of ground station support by the NASA Jet Propulsion Laboratory Deep-Space Network. A recent communication (July 1990) from Dr. Kardashev indicates that the possibility of using a US hydrogen maser on Radioastron remains open. 


\section{F. KEY SOVIET RESEARCH PERSONNEL AND FACILITIES}

A list of key Soviet personnel and facilities involved in research on hydrogen masers, cesium beam devices, rubidium gas cells, and rubidium masers is provided in Table III.3. 
Table III.3

KEY SOVIET RESEARCH PERSONNEL AND FACILITIESATOMIC FREQUENCY STANDARDS

( ${ }^{*}$ indicates leading personalities)

\section{HYDROGEN MASERS}

Physics Institute im. P. N. Lebedev, AS USSR, Moscow
N. G. Basov
A. A. Ul'yanov
G. A. Yelkin
G. M. Strakhovskiy
L. P. Yelkina
V. M. Tatarenkov

Physical-Technical and Radio Engineering Measurements All-Union Scientific Research Institute, Mendeleyevo (VNIIFTRI)
G. A. Yelkin
L. P. Yelkina
B. P. Popov
V. I. Purtov

A. F. Sh_ierbinin

A. V. Vishina

B. A. Gaygerov

N. D. Zhestkova

V. A. Paramzin

G. P. Kurnikov

G. Kryukov

S. B. Pushkin

Gor'kiy R\&D Instrument Making Institute, Gor'kiy

N. A. Demidov

B. A. Sakharov

Scientific Production Association (NPO) "Quartz," Gor'kiy
A. A. Ul'yanov
B. P. Fateyev
N. A. Demidov
Y. P. Sharov
A. A. Bogdan
A. A. Belyayev
Ye. M. Yezhov
S. A. Kozlov
A. I. Lavrov
S. V. Polvalyasko
V. A. Logachev
V. L. Simanskiy
G. M. Chernov
V. Yu. Maksimov
A. A. Ul'yanov
M. Yu. Fedotov

Leningrad Radiotechnical Scientific Research Institute, Leningrad

Yu. G. Gouzhva 
Table III.3

KEY SOVIET RESEARCH PERSONNEL AND FACILITIES-

ATOMIC FREQUENCY STANDARDS (cont'd.)

\section{CESIUM BEAM DEVICES}

Physical-Technical and Radio Engineering Measurements All-Union

Scientific Research Institute, Mendeleyevo (VNIIFTRI)

Yu. G. Abashev*

V. K. Kurakin

R. A. Valitov

G. F. Voronin

I. V. Chernyskev

N. N. Raskatov

V. I. Purtov

V. G. Il'in

N. A. Telpuchovskiy

V. V. Sazhin

S. B. Pushkin*
V. K. Yegorov

V. A. Maslov

L. A. Budkin

L. I. Polyakov

M. N. Penenkov

V. N. Baryshev

G. A. Yelkin*

Yu. V. Timofeyev*

Yu. V. Runov

A. A. Ul'yanov*

Leningrad Radiotechnical Scientific Institute, Leningrad

Yu. G. Gouzhva

\section{RUBIDIUM GAS CELLS AND RUBIDIUM MASERS}

Leningrad Polytechnic Institute im. M. I. Kalinin, Leningrad
M. B. Gornyy
D. L. Markman
G. M. Smirnova
B. G. Matisov
V. I. Khutorshchikov

Affiliation Unknown
A. Ya. Leykin
B. Yu. Linker
V. A. Dukhov
E. V. Kulagin
A. I. Samoylovich
A. P. Sheronov
B. A. Pavlova
M. P. Bespalova
V. Kalaytanov
Ye. Boriskin

I. M. Sokolov 


\section{CHAPTER III: ATOMIC FREQUENCY STANDARDS REFERENCES}

Abashev, Yu. G., V. N. Baryshev, G. A. Yelkin (Elkin), Yu. V. Timofeyev (Timofeev), L. I. Polyakova (Poliakova), S. B. Pushkin, V. Yu. Runov, and A. A. Ul'yanov (Ulianov), "Metrological Analysis of Type MTs-3 Cesium Frequency Reference," Meas. Tech., 29, 2(1986), 97-98.

Abashev, Yu. G., and N. N. Raskatov, "The Emission Activation Energy of Ions of Potassium and Sodium from Single Crystals of Tungsten," Meas. Tech., 17, 5(1974), 686-688.

Abashev, Yu. G., N. N. Raskatov, and I. V. Chernyshev, "Measuring the Background Ionic Emission of Tungsten Monocrystals Used in Quantum Frequency Standards," Meas. Tech., 16, 10(1973), 14711474 .

Abashev, Yu. G., G. F. Voronin, and R. A. Valitov, "Velocity Distribution in ar, Atomic Beam," Meas. Tech., 15, 11(1972), 1679-1681.

Abashev, Yu. G., G. A. Yelkin (Elkin), and S. B. Pushkin, "Primary Cesium Time and Frequency Standards," Meas. Tech., 26, 12(1983), 996-999.

Abashev, Yu. G., L. P. Yelkira (Elkina), and S. B. Pushkin, "The Main Characteristics and the Results of Long-Term Comparisons Between Primary Cesium Beam Standards and Ensemble of Hydrogen Clocks of the National Time Standard," IEEE Trans. Instrumentation and Measurement, IM-36, 2(1987), 627-629.

Baydakov (Baidakov), I. G., S. B. Pushkin, and V. P. Titov, "Securing L'nanimity of Time and Frequency Measurements in the USSR at the Highest Section of the Reference System," Meas. Tech., $16,1(1973), 60-63$.

Belyayev (Beliaev), A. A., N. A. Demidov, S. A. Kozlov, V. A. Logachev, S. V. Polvalyashko, V. L. Simanskiy (Simanskii), and A. A. Ul'yanov (Ulianov), "Experimental Investigation of Passive Hydrogen Frequency Standards," Meas. Tech., 27, 3(1984), 224-226.

Belyayev (Beliaev), A. A., N. A. Demidov, B. A. Sakharov, V. Yu. Maksimov, M. Yu. Fedotov, and A. E. Yampol'skiy (Iampolskii), "Ch1-76 Small-Size Passive Hydrogen Frequency and Time Standard," Meas. Tech., 30, 8(1987), 767-772.

Belyayev (Beliaev), A. A., and V. A. Savin, "Calculation and Analysis of Frequency Properties of Special Axisymmetric Resonators of Hydrogen Quantum Frequency Discriminators," Meas. Tech., 30, 2(1987), 154-157.

Bespalova, M. P., and A. I. Pikhtelev, "Apparatus for Producing Gas Mixtures," Otkrytiya, izobreteniya, 46(1983), 150.

Bogdan, D. A., N. A. Demidov, Ye. M. Yezhov (Ezhov), A. I. Lavrov, V. A. Logachev, B. A. Sakharov, G. M. Chernov, A. A. Ul'yanov (Ulianov), B. P. Fateyev (Fateev), and Yu. P. Sharov, "A Hydrogen Frequency Standard," Meas. Tech., 17, 11(1974), 1701-1704.

Dubkov, V. A., E. V. Kulagin, A. I. Pikhtelev, and A. P. Sheronov, "Measurements of the Instability Characteristics of Quantum Frequency Standards," Meas. Tech., 27, 2(1984), 136-138. 
Gaygerov (Gaigerov), B. A., "Automatic Phase Tuning Circuit with Controlled Phase Inverter," Meas. Tech., 15, 11(1972), 1662-1666.

Gaygerov (Gaigerov), B. A., "Automatic Stabilization of the Magnetic Field in a Hydrogen Generator," Meas. Tech., 16, 3(1973), 464-465.

Gaygerov (Gaigerov), B. A., "Hydrogen Masers with Automatically Tuned Resonators," Meas. Tech., 22, 9(1979), 1082-1086.

Gaygerov (Gaigerov), B. A., F. S. Rusin, N. I. Pol'nikov, V. P. Sysoyev (Sysoev), and S. N. Ovchinnikov, "Transportable Quantum Clocks Based on a Hydrogen Generator with a Small Resonator," Meas. Tech., 32, 4(1989), 309-311.

Gaygerov (Gaigerov), B. A., and G. A. Yelkin (Elkin), "Automatic Tuning of the Resonator of the Hydrogen Frequency Standard," Meas. Tech., 11, 6(1968), 839-840.

Gaygerov (Gaigerov), B. A., G. A. Yelkin (Elkin), L. P. Yelkina (Elkina), N. D. Zhestkova, G. P. Kurnikov, and B. P. Popov, "Hydrogen Maser as a Quantum Frequency Standard," Meas. Tech., 15, 11(1972), 1640-1643.

Gaygerov (Gaigerov), B. A., G. A. Yelkin (Elkin), and N. D. Zhestkova, "Fundamental Directions of Hydrogen Generator Development," Meas. Tech., 26, 12(1983), 1000-1003.

Gaygerov (Gaigerov), B. A., and L. P. Yelkina (Elkina), "Method of Tuning Resonator of a Hydrogen Generator," Meas. Tech., 19, 11(1976), 1589-1590.

Gaygerov (Gaigerov), B. A., L. P. Yelkina (Elkina), and S. B. Pushkin, "Metrological Characteristics of a Group of Hydrogen Clocks," Meas. Tech., 25, 1(1982), 23-25.

Gaygerov, B. A., S. B. Pushkin, and F. S. Rusin, "Hydrogen Generator," Otkrytiya, izobreteniya, 22(1986), 243.

Gornyy (Gornyi), M. B., B. G. Matisov, G. M. Smirnova, and V. I. Khutorshchikov, "Short-Term Stability of Rubidium Frequency Standards," Sov. Phys.-Tech. Phys., 32, 4(1987), 448-452.

Gouzhva, Y. G., B. N. Balyashnikov, V. V. Korniyenko, I. G. Pushkina, V. S. Shebshayevich, V. I. Denisov, and A. T. Reutov, "National and International-Wide Prospects of Future Improvements of Position Location and Time Synchronization Aids," Proc. XXII Annual Precise Time and Time Interval (PTTI) Applications and Planning Mtg., (NASA Conference Publication No. 3116), 1990, 525.

Ivanov, N. E., V. A. Salishchev, and A. O. Kupriyanov, "Some Aspects of Space Navigation System GLONASS: Application in the Interest of Time and Frequency Determination," Proc. 4th European Frequency and Time Forum, 13-15 March 1990 (Neuchâtel, Switzerland).

Kalaytanov (Kalaitanov), V., and Ye. Boriskin, "Frequency and Time Standard Ch1-74," Technology and Armaments, 11(1984), 45.

Kaminskiy (Kaminskii), V., and A. Nikulin, "Metrological Support in the Field," Tekhnika $i$ vooruzheniye, 11(1984), 45 (in Russian).

Korobov, V. K., V. G. Il'in, and S. B. Pushkin, "New State Standard of Time and Frequency, and Methods of Realizing its Accuracy," Meas. Tech., 19, 10(1976), 1444-1449. 
Kurakin, V. K., "Beam Intensity at the Exit of an Atomic-Beam Tube," Meas. Tech., 15, 11(1972), 1682-1684.

Rusin, F. S., B. A. Gaygerov (Gaigerov), and S. F. Ponomarenko, "Calculation of a Cylindrical Cavity Partially Filled with a Dielectric," Meas. Tech., 28, 5(1985), 396-398.

Vishina, A. V., and B. A. Gaygerov (Gaigerov), "Hydrogen Time and Frequency Standards with Redundancy," Meas. Tech., 19, 10(1976), 1460-1461.

Yelkin (Elkin), G. A., Yu. G. Abashev, V. V. Varyshev, and V. I. Purtov, "Cesium Atomic-Beam Frequency Standards," Meas. Tech., 26, 5(1983), 375-378.

Yelkin (Elkin), G. A., and V. I. Purtov, "Automatic System for Comparing the Frequency of a [Quantum] Store with the Transition Frequency of Cesium Atoms," Meas. Tech., 19, 10(1976), 14561460.

Yelkin (Elkin), G. A., and N. D. Zhestkova, "Magnetic Displacement and the Effect of Linear Transitions on a Hydrogen Generator's Frequency," Meas. Tech., 16, 10(1973), 1466-1468.

Yelkin (Elkin), G. A., and N. D. Zhestkova, "Collision-Dependent Frequency Shift in an AtomicHydrogen Clock," Meas. Tech., 22, 9(1979), 1076-1078.

Yelkin (Elkin), G. A., N. D. Zhestkova, and G. P. Kunikov, "Error Reproduction of the Frequency of Undisturbed Transition of the Hydrogen Atom," Meas. Tech., 16, 3(1973), 394-397.

Yelkin (Elkin), G. A., N. D. Zhestkova, and V. A. Paramzin, "Measuring Wall-Induced Frequency Shift in a Hydrogen Oscillator," Meas. Tech., 20, 10(1977), 1453-1455.

Zhestkova, N. D., G. A. Yelkin (Elkin), and V. I. Zemskova, "Hydrogen Frequency Standard and Undisturbed Frequency-Transition Evaluation," Meas. Tech., 19, 10(1976), 1461-1463.

\section{ADDITIONAL WORKS CONSULTED}

Abashev, Y. G., V. N. Baryshev, G. A. Yelkin (Elkin), and Y. N. Yakovlev (Iakovlev), "Metrological Parameters of the Primary Cesium Beam Frequency Standards in the National Time and Frequency Standard of the USSR," Frequency Standards and Metrology, ed., A. DeMarchi, Berlin: Springer-Verlag, 1989, 374.

Alekseyev (Alekseev), V. A., and I. I. Sobel'man, "Influence of Collisions of Atoms with Walls of a Storage Chamber on the Emission Frequency of a Hydrogen Maser," Sov. J. Quantum Electron., 15, 1(1985), 3-14.

Belov, V. N., A. G. Gevorkyan (Gevorkian), and G. G. Yushina (Iushina), "Determination of Cesium Saturated Vapor Density in the 304-364 K Temperature Range," Opt. Spectrosc., 63, 3(1987), 414415.

Belyayev (Beliaev), A. A., and V. A. Savin, "Calculation and Analysis of Frequency Properties of Special Axisymmetric Resonators of Hydrogen Quantum Frequency Discriminators," Meas. Tech., 30, 2(1987), 154-157. 
Belyayev (Beliaev), A. A., N. A. Demidov, S. A. Kozlov, V. A. Logachev, S. V. Polvalyashko (Polvaliashko), V. L. Siamankiy (Siamanskii), and A. A. Ul'yanov (Ulianov), "Experimental Investigation of a Passive Hydrogen rirequency Standard," Meas. Tech., 27, 3(1984), 224-226.

Bockhov, B. A., B. A. Gaygerov (Gaigerov), V. P. Korolev, and F. S. Rusin, "Hydrogen Oscillator with a Small Resonator," Radiotekhnika i elektronika, 26, 1(1981), 2471-2472 (in Russian).

Demidov, N. A., E. M. Yezhov (Ezhov), A. A. Ul'yanov (Ulianov), and V. A. Fedorov, "Frequency Shifts of a Hydrogen Generator Due to Hydrogen Atom Collisions with Storage Flask Walls," Meas. Tech., 20, 7(1977), 1000-1002.

Il'in, V. G., S. B. Pushkin, A. Vavr, and E. Graf, "The Radiological Characteristics of Cesium," Meas. Tech., 10(1979), 28-29.

Pushkin, S. B., G. A. Yelkin (Elkin), and G. A. Gaygerov (Gaigerov), "Investigation of the Metrological Characteristics of the State Primary Standard of Time and Frequency, USSR," Meas. Tech., $32,4(1989), 304-308$.

Skobel'tsyn, D. V., ed., "Quantum Electronics in Lasers and Masers," Proc. (Trudy) of the P. N. Lebedev Physics Institute, 31, (1968).

Urbanovich, S. I., "Pressure Dependence of the Hyperfine Structure of Hydrogen," Opt. Spectrosc., $42,5(1977), 467-469$.

Yelkina (Elkina), L. P., G. A. Yelkin (Elkin), and G. M. Strakhovskiy (Strakhovskii), "Measurement of the Frequency Drift of a Hydrogen Standard Owing to Atomic Impacts on the Flask Wall," Meas. Tech., 11, 6(1968), 841-842.

Zhestakova, and G. A. Yelkin (Elkin), "Subject of a Nonuniform Magnetic Field on a Hydrogen Maser," Meas. Tech., 22, 9(1979), 1079-1981. 


\section{CHAPTER IV \\ NEW AND EMERGING FREQUENCY STANDARD TECHNOLOGIES}

\section{A. SUMMARY}

There are a number of new technologies that may have a significant impact on frequency standards in the near future. The use of lasers for state preparation and resonance detection is likely to significantly improve the stability of cesium and rubidium standards. Stabilized lasers show promise because of their very high frequencies relative to linewidth. Very narrow resonances obtainable on microwave transitions in trapped ions have already been incorporated into prototype frequency standards that compete favorably with hydrogen standards. They are likely to dominate the market for long-term freciuency stability during the next decade. Techniques for cooling, collimating, and trapping ions and neutral atoms using lasers show considerable promise for future frequency standard development. Cryogenic hydrogen masers offer substantial improvements over conventional hydrogen standards. Cryogenic dielectric crystal oscillators show promise of providing clean, stable signals needed for frequency synthesis, and as flywheel frequency standards to be used in conjunction with atomic standards having very good long-term stabilities.

The Soviet participation in these areas is significant, but uneven. Soviet researchers have made important contributions to theoretical developments in all of these areas. Their efforts to produce laser frequency standards using saturated absorption techniques are more strenuous and more successful than any efforts in the West. They are major players in the use of laser light to manipulate beams of atoms, especially the collimation of atomic beams. They are leaders in the development of cryogenic single-crystal sapphire resonators. On the other hand, they are lagging in the application of laser cptical pumping techniques to improve cesium and rubidium standards. There is no evidence of experimental work on trapping ions or neutrals in low-frequency electromagnetic traps, and there does not appear to be any work with cryogenic hydrogen masers. The historical research interests of the various Soviet laboratories and the tastes of their directors surely play a role. The availability of certain materials and lasers may also be important. 


\section{B. OPTICALLY PUMPED AND OPTICAL STANDARDS}

Applications of laser optical pumping techniques to the established cesium and rubidium microwave standards promise significant improvements in those microwave standards in the near future. The Soviet Union has been very active in theoretical work, and there is considerable experimental work reported in the published literature. The Soviet rate of progress depends somewhat on developing or importing better stabilized solid-state lasers. Stabilized lasers are being developed in the Soviet Union as optical frequency standards. The Soviet emphasis on optical frequency standards is much greater than that of any other country, compared to their overall efforts in frequency standards. Soviet applications of laser optical pumping to microwave standards and the development of stabilized laser frequency standards are sufficiently differentiated, both in the physics they use and in the personnel involved, that they are presented here in separate subsections.

\section{Optically Pumped Microwave Standards}

\section{a. Summary}

The Soviet Union has performed a fair amount of theoretical work on optical pumping, but little experimental work or building of equipment, with the exception of passive optically pumped rubidium gas cell standards. There is some work being conducted on optically pumped primary cesium beam standards at the Physical-Technical and Radio Engineering Measurements All-Union Scientific Research Institute (VNIIFTRI). However, other than in theory, the Soviet Union appears to be lagging the United States in this important area. The Soviet work on optically pumped microwave standards can be characterized as displaying:

- excellent theoretical capabilities;

- limited access to high-quality foreign semiconductor diode lasers;

- inferior semiconductor diode lasers that are available only in limited quantities; and

- little experimental work or equipment. 


\section{b. Introduction}

Optical pumping is not new either in concept or in application. It was originally described in the 1950s, and the first applications to frequency standards appeared in the early 1960s. By 1965, the optically pumped rubidium gas cell standard was already highly developed. A relatively new and emerging concept is the use of lasers for optical pumping, in particular, the use of stabilized semiconductor solid-state injection lasers. The rapid development of reliable, longlife gallium-arsenic'e injection lasers has had a strong influence, since their wavelength is in a range of application to both cesium and rubidium standards.

In general, optical pumping can be described as the use of the interaction of optical radiation with quantum systems to obtain a non-equilibrium population distribution among the energy levels. It can be used to prepare or maintain a suitable difference between the populations of energy levels used in microwave standards that would normally have almost equal populations at ordinary temperatures due to the smallness of $\mathrm{hv}$, the difference between energy levels compared to the thermal energy $k T$. Here, $h$ is Planck's constant and $v$ is the transition frequency, which is in the microwave domain. Optical pumping can also be used as a means to detect microwave transitions.

A simple, three-level pumping scheme that corresponds to what is actually done in many cases is presented in Figure IV.1. With no applied optical signal, there is virtually no population of level $E_{3}$ at ordinary temperatures because it is so much higher in energy than $E_{1}$ and $E_{2}$. If an optical signal with frequency corresponding to the differences in energy between levels $E_{3}$ and $E_{1}$ is applied to the system, then transitions from $E_{1}$ to $E_{3}$ will be made. It is assumed that the lifetime for spontaneous emission from $E_{3}$ is very short, so the atoms in that level decay radiatively very quickly, with roughly equai probabilities to levels $E_{1}$ and $E_{2}$. This process is called pumping from $E_{1}$. The lifetime for spontaneous transitions from $E_{2}$ to $E_{1}$ is assumed to be very long, as is the case with the magnetic dipole transitions used in most of the microwave frequency standards. With no applied microwave radiation, the atoms will end up in level $E_{2}$ as only level $E_{1}$ is being pumped. If a microwave signal with frequency corresponding to the difference in energy between $E_{2}$ and $E_{1}$ is applied, transitions from $E_{2}$ to $E_{1}$ will occur, and these can be detected in many ways. If a sufficiently high $Q$ cavity res- 
onant at the microwave transition frequency is placed around the system, it could oscillate as a maser at the transition frequency. This is the principle of the optically pumped rubidium maser.

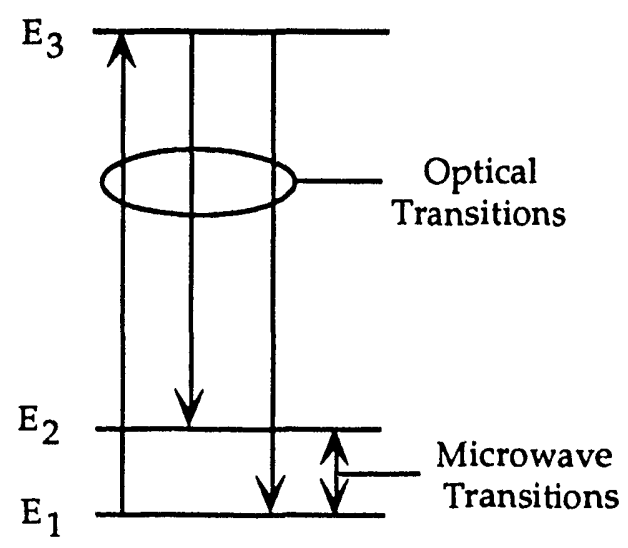

Atoms are pumped by laser light from $E_{1}$ to $E_{3}$, from which they decay spontaneously to both $E_{1}$ and $E_{2}$, populating $E_{2}$ at the expense of $E_{1}$. Microwave transitions drive atoms back to $E 1$, increasing the optical transition rate and the amount of fluorescence.

Figure IV.1

Three-Level Optical Pumping

Optical pumping with a laser improves the performance of cesium standards. It can greatly improve the signal-to-noise ratio and, consequently, the short-term frequency stability. Replacing the state selecting magnets with optical state selection and optical detection by pumping can improve the homogeneity of the necessary small magnetic $C$ field in the microwave transition and drift regions, and thus improve the accuracy. In addition, the response of adjacent microwave transitions (Zeeman transitions) can be made to be very symmetric, in contrast to the situation for magnetic state separation. This also leads to improved accuracy. Most frequency standards laboratories are pursuing this approach.

Laser pumping of rubidium gas cell devices can improve their short-term frequency stability by a considerable amount. One important characteristic of the laser pumping source is its noise. The performance of many of the optical detec- 
tion methods is limited by the amplitude noise of the laser. Incidental frequency modulation at low frequencies can also be a problem.

Optical pumping also is being used widely in other types of frequency standards, such as trapped ion devices and optical standards. These are discussed in other sections.

\section{c. Discussion of Soviet Work}

Early Soviet experiments on optical pumping with diode lasers used current and temperature stabilization instead of external cavity stabilization (Budkin et al., 1984a). The reported laser linewidths were roughly $50 \mathrm{MHz}$ but, in looking at the D2 absorption line of cesium, the resolution was about $150 \mathrm{MHz}$. This indicated that, if the full capability of the bare diode laser were to be achieved, the temperature and current regulation needed to be improved. The Soviet Union was lagging the United States at this time.

Considerable Soviet work was performed on the theory of optical pumping in three-level systems. There were a number of detailed articles using the density matrix formalism, some including the effects of the motion of the atoms and the spectral width of the pumping radiation (Gornyy et al., 1984; Budkin et al., 1985).

Light frequency shifts (due to simultaneous irradiation of the system with both the optical pump signal and the microwave signal of interest) were already well known in the Soviet Union and were studied both theoretically and experimentally (Budkin et al., 1984b). Dependence of the pumping light on spectral width was treated theoretically, and experimental results were reported for large spectral widths. Experimental results for narrow widths were also given, but these were attributed to a French group. This is a clear indication that the Soviet Union was lagging in laser technology.

A numerical calculation on the efficiency of optical pumping of cesium was performed by Yegorov and Maslov (1983). A number of cases were treated, including no mixing, as well as complete mixing of the optically excited states. Techniques for obtaining symmetric populations about the $\mathrm{m}_{\mathrm{F}}=0$ sublevels were also discussed. The well-known, two-laser scheme for completely populat- 
ing the $\mathrm{F}=4, \mathrm{~m}_{\mathrm{F}}=0$ level was cited as being highly desirable for a frequency standard application. This was good theory, but no experimental results were published.

A laser pumped gas cell frequency discriminator was studied theoretically and experimentally by Budkin et al. (1984c). The authors showed that, in principle, it is possible to obtain a considerably improved signal-to-noise ratio with laser pumping in rubidium gas cell frequency standards. Their experiments showed some improvement over the usual spectral lamp pumped standards, but not the improvements expected from the theory. There was excess noise, possibly due to multimoding in the pump laser and extra noise in some of the electronics. This is an important area of work that is recognized worldwide.

Budkin et al. (1988) described experiments on double resonance in cesium vapor in a gas cell with optical pumping. A low-frequency signal for exciting the Zeeman transitions, as well as the microwave signal, was applied. Optical pumping was performed by a semiconductor injection laser without stabilization other than that of current and temperature. This double resonance technique can make a frequency discriminator for frequency standards with improved signalto-noise ratio, but it is somewhat complicated. In addition, there could be frequency pulling.

In optical pumping, the Soviet Union is doing a good job with theory, but is lagging the West in experimental work and practical applications. Soviet researchers are suffering from the lack of availability of good injection lasers, and are lagging in stabilized laser sources.

\section{d. Projections for the Future}

The Soviet ability to make good injection lasers will improve over the next few years, which will benefit their optical pumping efforts. These lasers are very important for telecommunications, particularly in the 1.3- and 1.55-micron wavelengths used in fiber-optic systems. The improved frequency stability and accuracy that could be achieved with optical pumping will have important consequences in both navigation and communication, and is an important area of work worldwide. 


\section{Stabilized Laser Optical Standards}

\section{a. Summary}

Soviet scientists are very active in the field of optical frequency standards, especially methane-stabilized helium-neon ( $\mathrm{He}-\mathrm{Ne}$ ) lasers. They entered the field of optical frequency standards competitively from the start and have continued to be competitive with the rest of the world. The Soviet Union was among the first to apply saturated absorption spectroscopy to the development of an optical frequency standard, and among the first to develop a frequency synthesis chain to link optical frequencies to microwave frequencies. Soviet researchers apparently have chosen to place more emphasis on this technology than on other emerging frequency standard technologies. They have taken the lead in the development of "supernarrow" saturated absorption resonances for frequency standard use and are active in developing improved frequency synthesis chains. Overall, the Soviets are conducting as much or more work on optical frequency standards than anyone in the world, and their work is first rate. Characteristics of the current Soviet research effort include:

- commitment to developing an optical frequency standard;

- involvement of several laboratories;

- continuing development of very large cell methane-stabilized lasers;

- continuing exploration of other supernarrow resonance techniques;

- continuing development of better links from optical-to-microwave frequencies; and

- development of a portable optical standard.

\section{b. Introduction}

The principles of gas laser operation are illustrated in Figure IV.2, using the He-Ne gas laser developed in 1960 at AT\&T Bell Laboratories by Javan and colleagues as an example. An electric discharge in a mixture of helium and neon gas contained in a cylindrical glass laser tube excites helium atoms from the ground state to the excited metastable $2^{3} \mathrm{~S}$ and $2^{1} \mathrm{~S}$ states, but has little effect on the neon atoms. For simplicity, only the processes involved in stimulating laser 
oscillation at $3.39 \mu \mathrm{m}$ are illustrated. In that case, excitation is transferred from the $2^{1} \mathrm{~S}$ level of excited helium atoms to the very nearly coincident $3 \mathrm{~s}$ energy level of neon atoms by collisions between helium and neon atoms. Neon atoms are stimulated by resonant light to make transitions from the $3 \mathrm{~s}$ level to the $3 p$ level, emitting light at wavelength $3.39 \mu \mathrm{m}$, which reinforces the resonant light. The lower $3 p$ level is emptied by spontaneous decay to the 1 s level, which decays to the ground state via wall collisions. The emitted light passes through Brewster angle windows and is reflected back and forth in an optical "cavity" between mirrors at either end of the laser, storing the resonant light so as to stimulate further transitions in newly excited neon atoms. If the rate at which energy is transferred to excited neon atoms exceeds the loss of energy due to relaxation processes and leakage of light from the cavity, self-sustained laser action builds up an optical standing wave between the mirrors, a fraction of which escapes through a partially silvered mirror as coherent light output.

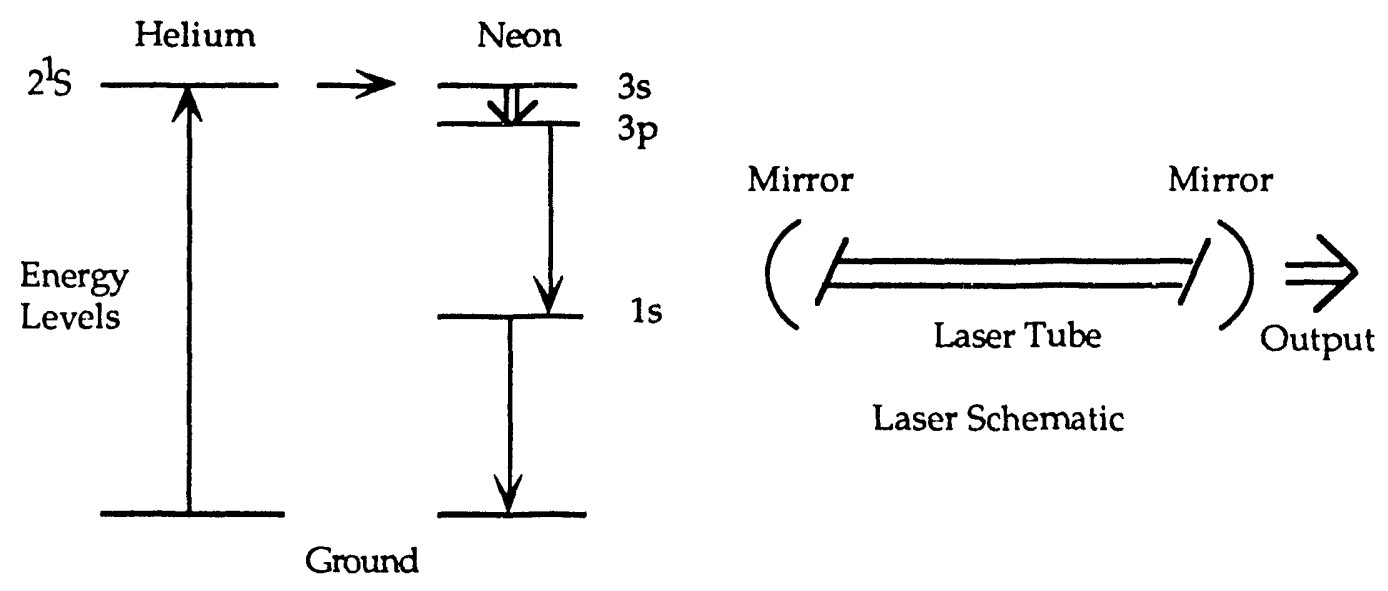

Figure IV.2

Helium-Neon Laser

The gain profile of such a laser is broad because of the Doppler effect: moving atoms emit radiation at frequencies shifted fractionally by $\mathrm{v} / \mathrm{c}$, leading to a velocity dependent gain profile with a width determined by the range of thermal velocities. In addition, the center of the gain profile is shifted by collisions, leading to frequency instabilities in the $10^{9}$ domain. However, the laser frequency 
can be stabilized on an external reference transition (as illustrated in Figure IV.3) in the case of saturated absorption in methane. ${ }^{1}$

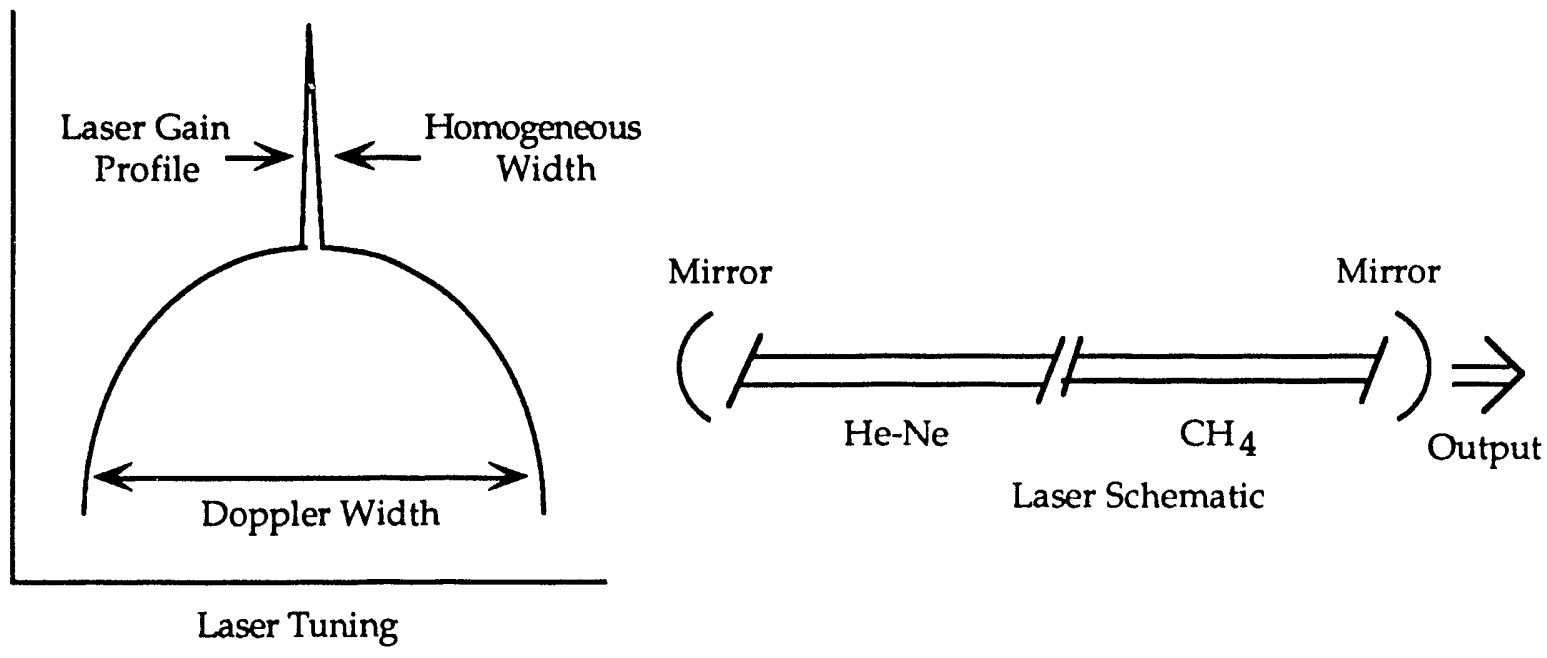

Figure IV.3

Methane-Stabilized Laser

A second cell filled with methane at millitorr pressures absorbs laser light at a rate that reduces the laser gain just to unity at the ambient light level. If the laser is slightly detuned from the center of the methane molecular absorption line (in this case the P[7] line of the $v_{3}$ band), transitions occur only in those molecules whose frequencies are Doppler shifted into resonance with the laser light by their motion relative to the two counter-propagating light waves that make up the laser standing wave. As the laser frequency tunes closer to the molecular resonance, molecules undergoing transitions have smaller and smaller longitudinal velocities, until at line center, both counter-propagating waves interact with the same zero-longitudinal-velocity subgroup of molecules, saturating the transition and reducing the total number of resonant absorbers. This produces a peak in the emitted power centered at zero mistuning and having a resonance width determined only by the homogeneous broadening of the molecular transition. The characteristics of this particular transit on, which led

$1 \quad$ R. L. Barger and J. L. Hall, "Pressure Shift and Broadening of Methane Line at $3.39 \mu$ studied by Laser-Saturated Molecular Absorption," Phys. Rev. Lett., 22 (1969), 4-8. 
to its initial choice and continued popularity, include relatively long natural lifetime and, consequently, narrow linewidth. Because of its near coincidence with the 3.39- $\mu \mathrm{m}$ He-Ne laser transition frequency, this transition has a large absorption coefficient and therefore it can be easily saturated. It is relatively insensitive to stray fields and other systematic effects.

Gas cells filled with iodine $\left(\mathrm{I}_{2}\right)$, sulphur hexafluoride $\left(\mathrm{SF}_{6}\right)$, and osmium tetraoxide $\left(\mathrm{O}_{\mathrm{s}} \mathrm{O}_{4}\right)$ are also used as saturated absorbers, but the best results have been obtained with methane $\left(\mathrm{CH}_{4}\right)$. Refinements of saturated absorption techniques such as separated optical fields, cooling the sample to $70^{\circ} \mathrm{K}$, selection of slowly moving molecules, and expansion of the laser beams in large absorption cells to provide long interaction times have produced "supernarrow" absorption profiles $\left(Q \approx 2 \times 10^{11}\right)$. High resonance $Q$ and large transition energy produce optical frequencies $(88.4 \mathrm{THz})$ with better short-term stabilities than the best current microwave standards (Bagayev et al., 1981). There are two principal obstacles to developing practical optical standards based on these techniques: (i) the stabilities degrade after averaging : mes of only 100 seconds or so due to various systematic effects, and (ii) the transfer of optical frequency stability down to the microwave region of interest to most frequency and time applications requires an extremely complex and expensive set of phase locked lasers and masers. The reliability of these chains has been poor, and stability is significantly degraded. For these reasons, the rest of the world has shifted its attention toward new types of microwave standards which offer even better performance and do not require the difficult transfer from the optical-to-microwave frequency domains. However, some progress in frequency transfer is possible using new techniques and improved crystal oscillators. Cooling and trapping particles using lasers may also benefit the development of optical frequency standards.

c. Discussion of Soviet Work

The first observations of a sharp saturated absorption peak above the Doppler profile of He-Ne laser output power were made by Lee and Skolnick ${ }^{2}$ at Perkin Elmer and by Lisitsyn and Chebotayev (1967) at the Semiconductor Physics Insti-

P. H. Lee and M. I. Skolnick, "Saturated Neon Absorption Inside a 6238- $\AA$ Laser," Appl. Phys. Lett., 10 (1967), 303-305. 
tute. The paper by Lisitsyn and Chebotayev concludes with a suggestion to try the molecular transition in methane at $3.39 \mu \mathrm{m}$. Soviet theoretical and experimental investigations of saturated absorption spectroscopy soon followed in parallel with work being conducted in the West. In 1978, Chebotayev published a benchmark review paper which is significant because it displays the early Soviet claim of precision comparison of two methane-stabilized He-Ne lasers with a frequency stability in the 10-15 domain for averaging times out to 100 seconds, and on that basis essentially sets the agenda for developing an optical frequency standard (Chebotayev, 1978).

In the meantime, N. G. Basov and coworkers at the Lebedev Physics Institute in Moscow achieved frequency stabilities of 5 parts in $10^{14}$ for 10-second averaging times using an He-Ne ring laser with a methane absorption cell (Basov et al., 1972). Another Soviet group active in the middle 1970s was headed by $\mathrm{Yu}$. S. Domnin, who is now at VNIIFTRI. In 1976, Domnin and coworkers reported measurements, using a variety of detectors, of the frequencies of lasers to be used in a chain of harmonic measurements to determine the absolute frequency of an He-Ne laser at $3.39 \mu \mathrm{m}$ (Domnin et al., 1976). More recently, this group has reported the absolute measurement of the frequency of a $\mathrm{CO}_{2}$ laser stabilized using $\mathrm{OsO}_{4}$ with a frequency stability of 3 parts in $10^{13}$ and a reproducibility of 3 parts in $10^{12}$ (Domnin et al., 1987). The same level is claimed for the absolute measurement of its frequency using a multiplier chain at the Soviet State Time and Frequency Standard. Although such lasers presently are not the best candidates for an optical standard, stabilized $\mathrm{CO}_{2}$ lasers can play an important role in frequency synthesis chains in which two stabilized $\mathrm{CO}_{2}$ lasers and a microwave source are sufficient to span the range from a few gigahertz to $150 \mathrm{THz} .^{3}$

The effects of collisions in saturated absorption were treated in a short theoretical paper by Alekseyev (1981), which reported both a broadening effect and a frequency shift that is small in the case of methane.

3 A. Clairon, O. Acef, C. Chardonnet, and C. J. Bordé, "State of the Art for High Accuracy Frequency Standards in the 28- $\mathrm{THz}$ Range Using Saturated Absorption Resonances of $\mathrm{OsO}_{4}$ and $\mathrm{CO}_{2}$," Proc. 4th Symp. on Frequency Standards and Metrology, Berlin: Springer-Verlag, $1989,212-221$. 
The use of stabilization by dispersion in saturated absorption rather than an intensity change was reported by Basov et al. (1981), who claimed an improved resolution and signal-to-noise level, as opposed to using the other technique in a cell the same size.

At the Third Symposium on Frequency Standards and Metrology at Aussois in 1981, Chebotayev presented his claims that methane-stabilized lasers allowed "transfer frequency characteristics of a highly stable laser in the frequency range from 0 to $10^{14}$ with no losses in accuracy" (Chebotayev, 1981). He described a measurement system for using a series of phase-locked lasers to transfer frequencies from $10^{14} \mathrm{~Hz}$ to microwave frequencies using various diode detectors. He justified his claim of no loss in accuracy by comparative stabilities consistent with the likely instability contributions from the rubidium microwave standard alone. A plot of the Allan standard deviation of two methane-stabilized lasers at $3.39 \mu \mathrm{m}$ compared directly with each other claims extravagantly low relative frequency instabilities, as low as a few parts in $10^{17}$ at $\tau=10$ seconds. These claims, the fact that the paper is entitled "Optical Time Scale," and the lack of stability data at longer averaging times where systematic effects inevitably degrade the stability, are all consistent with an interpretation of this paper as a propaganda piece, probably as much for home consumption as to impress the international frequency standard community present at the conference. At the same meeting, Chebotayev's colleagues described saturated absorption methane resonances narrower than $1 \mathrm{kHz}\left(\mathrm{Q} \approx 10^{10}\right)$ which were obtained using a large methane cell with a laser beam expander to increase the transit time of molecules through the optical field (Bagayev et al., 1981).

A more recent review by Bagayev and Chebotayev (1986) sums up their case for an optical frequency standard. Citing such recent methods for improving the linewidth as separated optical fields, two photon resonances, trapping and cooling, and selecting slow molecules, Chebotayev promises the "achievement of frequency stability on the order of $10^{-16}$ to $10^{-17}$." He also states

Simplification of the frequency synthesis scheme will permit developing optical standards which will be able to compete in practice with the best microwave time and frequency standards. The accuracy of frequency measurements in the optical range must reach values of $\approx 10^{-11}$, and the measurement technique itself must be simple and widely available. 
This review is significant both because of its comprehensive summary of Soviet work in this field, and because it displays a continuing Soviet commitment to developing an optical frequency standard, as opposed to, for example, putting more effort into the development of standards based on stored ions. Since that review, Bagayev et al. (1988) have reported reduced resonance widths obtained by optical selection of slowly moving methane molecules. At the Fourth Symposium on Frequency Standards and Metrology in Ancona in 1988, Bagayev and coworkers reported comparisons of their methane-stabilized optical standards to atomic hydrogen maser standards, indicating that the laser standards were superior to the hydrogen standards (Bagayev et al., 1989b). Again, however, the data were presented only for relatively short ( 0.1 to 1 second) averaging times. The group also reported on their results using optical selection of slow molecules with a large (4.5 meters long) methane absorption cell, and claimed to have obtained resonances as narrow as $100 \mathrm{~Hz}\left(\mathrm{Q} \approx 10^{11}\right)$ (Bagayev et al., 1989a).

Similar work continues at the Lebedev Physics Institute. In 1987, Basov and coworkers reported a stability of 2 parts in $10^{14}$ for $\tau=100$ seconds for a portable He-Ne optical clock (Basov et al., 1987). Workers using such clocks at VNIIFTRI now claim a frequency reproducibility of 3 parts in $10^{14}$, although colleagues at the National Research Council in Teddington (United Kingdom), who have studied these standards in-house, do not agree that the reproducibility is that good. At the Ancona Frequency Metrology Meeting, the Lebedev group reported a reproducibility of stationary lasers of 3 parts in $10^{13}$ and predicted that a reproducibility of a few parts in $10^{14}$ could be obtainable with better signal to noise (Gubin et al., 1989). At the Gordon Conference on Atomic Physics in the summer of 1989, Gubin described, in a poster session, the performance of a He-Ne laser stabilized with a 1.6 -meter methane absorption cell cooled to $70^{\circ} \mathrm{K}$ to achieve $Q \approx 2 \times 10^{11}$.

Preliminary results were given by Kurochkin et al. (1988) on resonances observed in $\mathrm{SF}_{6}$ in an internal absorption cell in a two-mode $\mathrm{CO}_{2}$ laser. Both intensity and dispersion resonances were observed. The intensity resonance width was $50 \mathrm{kHz}$. 
In addition to the work on $\mathrm{CO}_{2} / \mathrm{OsO}_{4}$ lasers, there is recent work on stabilized lasers, which are not direct candidates for optical frequency standards, but may play a role in developing frequency synthesis chains that can transfer the excellent short-term frequency stability of $\mathrm{He}-\mathrm{Ne} / \mathrm{CH}_{4}$ lasers from optical-tomicrowave frequencies. One example, the stabilization of semiconductor lasers by locking them to external Fabry Perot cavities, is reported by Akulshin et al. (1989) from the Lebedev Physics Institute. Another example from the same group is the stabilization of semiconductor lasers using saturated absorption resonances (Akulshin et al., 1988).

The Soviet Union leads the world in applications of spectroscopic techniques to achieve supernarrow resonances for optical frequency standard applications. There seems to be some weakness in Soviet attention to the crucial problem of translating the good short-term stabilities at optical frequencies down to microwave frequencies. However, there is recent evidence that Chebotayev and coworkers are beginning new work to improve their frequency chain (Zakharyash et al., 1989). In addition, the above mentioned work on other laser standards may aid the frequency synthesis problem. The most significant Soviet weakness is the lack of improved stabilities for averaging times greater than 100 seconds.

\section{d. Projections for the Future}

While most Western researchers are speculating that stored ions will provide the technology for the next generation of frequency standards, Soviet researchers seem to favor stabilized laser standards. Evidence of this can be seen in their strong efforts in that area, and also in the claims and predictions made in Chebotayev's review articles. Reverse evidence is provided by the lack of experimental work with stored ions, despite Chebotayev's occasional theoretical contributions in that area (see Section IV.C). Presently, the Soviet outlook for a practical optical standard that can compete favorably with the emerging stored ion standards is not very good, both because of instabilities at long averaging times and because of the difficulties in comparing optical frequencies with the frequencies of microwave standards. Breakthrough signs of significant milestones would be convincing evidence of improved stabilities for averaging times beyond 100 seconds, and evidence of improved synthesis of optical frequericies 
from microwave frequencies. Whatever the future of saturated absorption frequency standards as competitive frequency standards, the technology of stable lasers and optical frequency synthesis and measurement will have important applications to information transfer and storage at optical frequencies, an area of such technological importance as to have military and political significance.

Achievements to watch for include reproducibility of methane-stabilized lasers to a few parts in $10^{15}$ using supernarrow resonances, experiments with laser-cooled saturated absorbers, improved frequency synthesis chains, improved stability for $\tau>100$ seconds, and optical spectroscopy of trapped ions and atoms.

\section{TRAPPED AND COLD PARTICLE RESEARCH}

Frequency shifts in atomic standards due to collisions with buffer gases or storage container walls can be avoided by trapping the particles in electromagnetic fields, using either $\mathrm{DC}$ or radio frequency (RF) electromagnetic fields produced by electrodes, or optical electromagnetic fields produced by lasers. Electrons and ions are more easily trapped in electrode traps than neutral atoms and molecules because of the strong electric field force on charged particles. The first applications of trapping to frequency standards are electrode trapped ion standards, which are under development in most Western standards laboratories. Prototype commercial trapped ion standards are already being used in the United States. Laser cooling of electrode trapped ions to further reduce instabilities due to the second-order Doppler shifts shows promise of making trapped ion standards the state of the art in long-term frequency standards in the near future.

The Soviet Union has conducted significant theoretical work on trapping ions, but there is no evidence of experimental development of electrode trapped ion standards. Uncharged atoms and molecules must be precooled by laser cooling or cryogenic techniques before trapping in electrode traps because the electromagnetic forces are so much weaker. Alternatively, neutrals may be trapped in purely optical traps. Precision spectroscopy of trapped neutrals, prerequisite to any eventual development of trapped neutral frequency standards, is underway in several US and European laboratories. 
The Soviet Union has made substantial cortributions to the theory of laser cooling and trapping. There is significant experimental work in laser cooling and trapping of neutrals, however, no evidence of electrode traps for neutrals was found.

Finally, atomic hydrogen masers are operated at low temperatures using molecular solid and superfluid liquid helium storage surfaces. Again, the Soviet Union has made significant contributions to the theory, but there is no evidence of experimental work with cryogenic hydrogen masers. Trapped and cooled ions, cooled and trapped neutrals, and cryogenic hydrogen masers are discussed in the following subsections.

\section{Trapped and Cooled Ions}

\section{a. Summary}

Since the pioneering work of Hans Dehmelt demonstrated that charged particles can be stored for very long times in electromagnetic trapping fields, most standards laboratories in the West have been working on precision spectroscopy of stored ions and the development of trapped ion frequency standards. Prototype commercial mercury ion frequency standards at the US Naval Observatory (USNO) perform as well as or better than hydrogen maser standards for long averaging times and are now contributing to the USNO time scale. Innovations such as laser cooling of single trapped ions are expected to produce practical frequency standards with frequency stabilities in the 10-16 domain in the near future. There has been significant Soviet theoretical work on trapping and cooling particles, including ions, but there is no evidence of any experimental work with trapped ions. Apparently, the groups with experimental expertise that would lend themselves to either the fundamental physics of trapped ions or to the development of trapped ion frequency standards have chosen to work in other areas, such as cooling and collimating neutral atoms and laser frequency standards. 


\section{b. Introduction}

As illustrated in Figure IV.4, Penning traps use a r.niform magnetic field $\mathrm{B}_{0}$ to confine the ions in the radial direction, and a DC potential difference between ring and endcaps to confine them vertically.

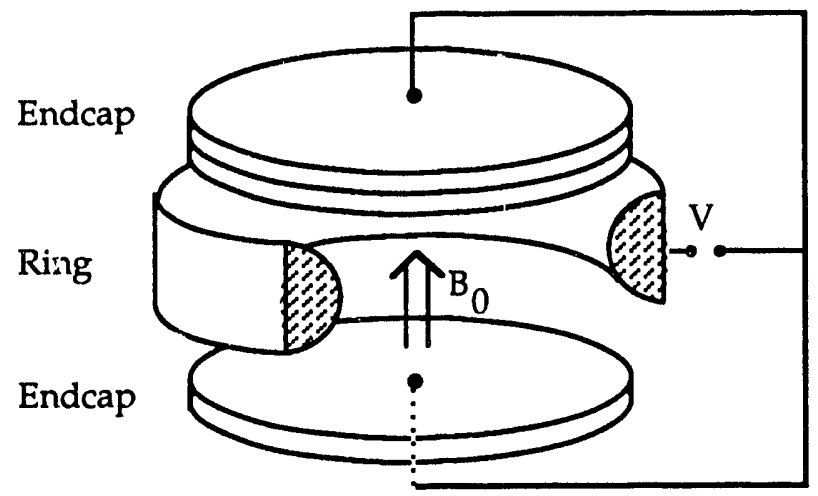

Figure IV.4

Schematic of an Ion Trap

For a Penning trap with inside dimensions of $1 \mathrm{~cm}$, typical values of magnetic field and potential difference are 1 Tesla and 1 volt, respectively. ${ }^{4}$ Radio frequency traps use a sinusoidal potential difference $V=V_{1}+V_{2}$ sin $\omega t$ without any magnetic field. The RF electric field causes the ions to oscillate with an amplitude proportional to the field strength, and with average force directed toward the center of the trap where the field is weaker. The effective potential well is approximately harmonic, so that the ions undergo secular motion with well-defined orbital frequencies, in addition to oscillating at the applied RF field frequency in the case of RF traps. Ions can be held in Penning and RF traps for several days.

W. M. Itano, J. C. Berquist, and D. J. Wineland, "Laser Spectroscopy of Trapped Ions," Science, 137 (1987), 612-617. 
Three prototype mercury ion frequency standards manufactured by HewlettPackard 5 are in operation at the US Naval Observatory. They use mercury 199 ions produced in an RF trap by bombardment with 2 electron volt electrons and cooled by collisions with helium background gas to a few hundredths of an electron volt. The ground-state hyperfine transition resonance is detected by optical pumping. The width of the ground-state hyperfine resonance, as detected by monitoring the 194-nm fluorescence intensity, is roughly $1.5 \mathrm{~Hz}$, or $\mathrm{Q} \approx 3 \times 10^{10}$ (see Figure IV.5). Long-term frequency stabilities for prototype standards are as good as or better than those of hydrogen maser standards. ${ }^{6}$

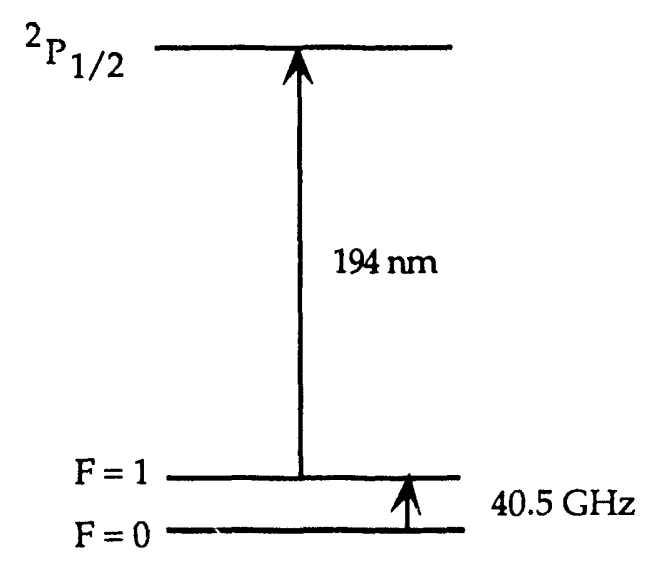

Optical radiation at $194 \mathrm{~nm}$ pumps atoms from the $\mathrm{F}=1$ ground state to the excited state, producing fluorescence as they decay back to the ground states. Microwave radiation at $40.5 \mathrm{GHz}$ pumps atoms from the $\mathrm{F}=0$ ground state to the $\mathrm{F}=1$ ground state, increasing the rate at which atoms are pumped to the excited state, thereby increasing the intensity of fluorescence when the $40.5 \mathrm{GHz}$ is on resonance.

\section{Figure IV.5 \\ Schematic of Mercury 199 Energy Levels}

5 L. S. Cutler, R. P. Giffard, and M. D. McGuire, "A Trapped Mercury 199 Ion Frequency Standard," Proc. 13th Annual Precise Time and Time Interval (PTTI) Applications and Planning Mtg., (NASA Conference Publication 2220, 1981, 563-578).

6 L. S. Cutler, R. P. Giffard, P. F. Wheeler, and G. M. R. Winkler, "Initial Operating Experience with a Mercury Ion Storage Frequency Standard," Proc. 49th Annual Symp. on Frequency Control, 1987, 12-19. 
The short-term stabilities of trapped ion frequency standards are limited by the signal-to-noise ratio, and therefore improve with larger numbers of trapped ions. The long-term stabilities are limited by the second-order Doppler shift, which is worse for larger numbers of ions because the space charge pushes the ions away from the center of the trap out to regions where their kinetic energy is larger. The second-order Doppler shift can be greatly reduced in Penning traps by laser cooling using the recoil force exerted on an ion when it absorbs a photon. Laser light with frequency tuned below the natural resonance frequency is absorbed preferentially by ions moving opposite to the direction of the light because the light frequency is shifted toward resonance by an amount proportional to the ion speed because of the Doppler shift. Absorbing a photon decreases the ion momentum by an amount equal to the photon momentum, and slows down the ion. Ions moving with the direction of the light are out of resonance with the laser light and are unaffected. The net effect of absorbing low-frequency photons and decaying spontaneously back to the ground states is to slow the ions to a limit set by the momentum of a single photon, on the order of $10^{4}$ times cooler than ions cooled by collisions with background gas (see Figure IV.6).

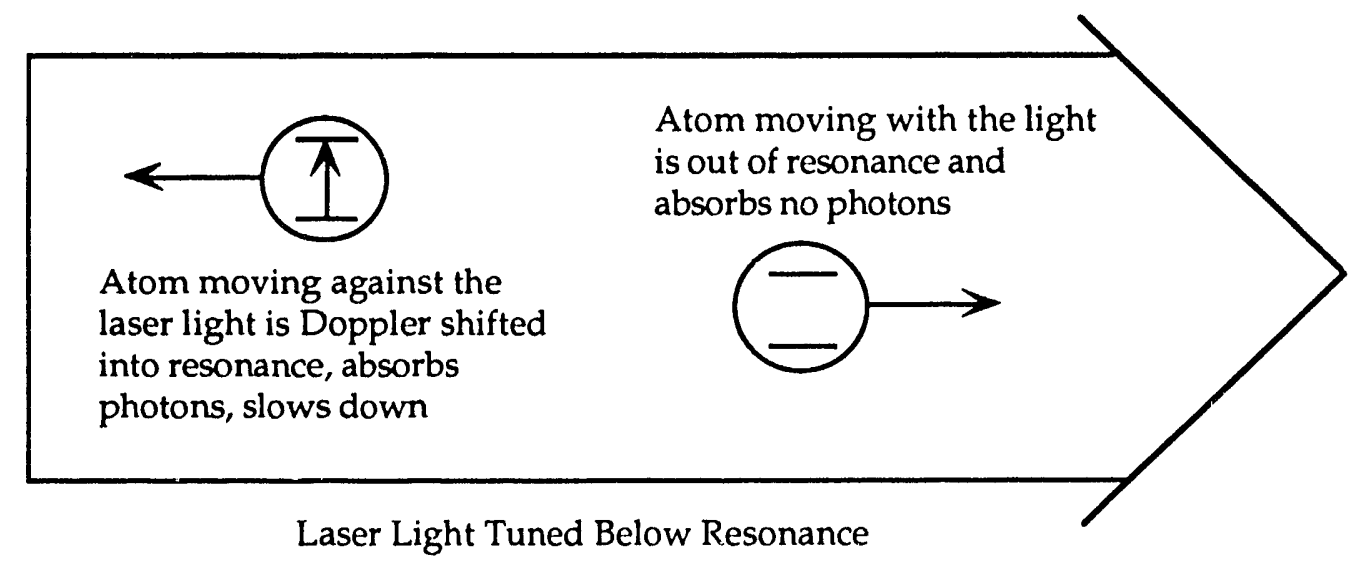

Figure IV.6

Laser Recoil Force Cooling

The potential precision improvement due to reducing the second-order Doppler shift by laser cooling suggests that practical frequency standards with 
long-term stabilities better than a few parts in $10^{16}$ or even $10^{17}$ may soon be available. ${ }^{7}$

c. Discussion of Soviet Work

Soviet physicists have made many contributions to the theory of lineshapes of particles moving in trapping potentials, including trapped ions. 8 In addition, they have made many contributions to the theory of laser cooling of trapped particles, including ions. 9 These theories are interesting because of the fundamental physics of collective effects at low temperatures, and they are also important for frequency standard applications. Consequently, it is potentially significant that there is no mention of experimental work with trapped ions in the published Soviet literature. If there is such work, it is probably not occurring in the laboratories conducting experimental work with laser-cooled neutral atoms, since that work is published in the Soviet literature and is even submitted in English to US journals.

\section{d. Projections for the Future}

Future Soviet development of trapped ion frequency standards is not likely, at least as evidenced in the published literature. This is consistent with commitments to improve conventional hydrogen maser standards and to develop an optical frequency standard. The absence of trapped ion frequency standard development may indicate that the Soviet Union will fall behind the United States and other Western nations during the next decade, when trapped ion frequency standards are likely to dominate long-term timekeeping. The appearance in the Soviet literature of reports on cooling trapped ions to new temperature limits might indicate a change of heart and new interest in developing trapped ion frequency standards.

7 D. J. Wineland, W. M. Itano, J. C. Berquist, J. J. Bollinger, F. Diedrich, and S. L. Gilbert, "High Accuracy Spectroscopy of Stored Ions," Frequency Standards and Metrology, ed., A. DeMarchi, Berlin: Springer-Verlag, 1989, 71-77.

8 Baklanov et al., 1979, 1981; Titov and Ulybin, 1982; Baklanov and Chebotayev, 1986.

9 Letokhov, 1968; Kazantsev, 1973; Vyatchanin, 1977; Letokhov and Minogin, 1981; Minogin and Rozhdestvenskiy. 1984: Kazantsev et al., 1985. 


\section{Cooled and Trapped Neutral Atoms}

\section{a. Summary}

As in many other areas, the Soviet Union has made significant theoretical contributions to the study of cooling and trapping neutral atoms. At the Spectroscopy Institute in Troitsk, one very active experimental group led by V. S. Letokhov has made a number of significant advances in the manipulation of atoms by laser-light forces, and a second experimental group led by V. I. Balykin is now active. Applications of laser cooling to improve optical frequency standards have been proposed by Soviet researchers, and there is some recent work on improving cesium beam standards. There is no evidence of trapping laser cooled atoms in electrode traps where long lifetimes and low Doppler shifts may lead to precision RF spectroscopy and to frequency standard applications.

\section{b. Introduction}

Forces exerted on atoms by laser light can be described as falling into roughly two classes. The first is the radiation pressure force (discussed in Section B.1 above and illustrated in Figure IV.6). Laser light at a frequency below that of an atcinic transition is Doppler shifted into resonance with the transition in an atom moving toward the laser beam. The atom is slowed down by absorbing the photon momentum and then recoils again when it decays by spontaneous emission. The photons emitted during spontaneous decay are emitted randomly in all directions, so that the net effect of successively absorbing photons from the laser beam and then decaying spontaneously is to slow down the atoms moving toward the laser beam. The radiation pressure force can be used to slow down and stop an atomic beam, provided that the atomic transition and the laser-light frequency are kept in resonance as the atoms slow down, either by "chirping" the laser frequency (Balykin et al., 1979), or by tuning the energy of the atomic transition by applying a magnetic field. ${ }^{10}$ The radiation pressure force due to multiple laser beams can be used to collimate atomic beams, as illustrated in Figure IV.7. (1982), 596-599. 


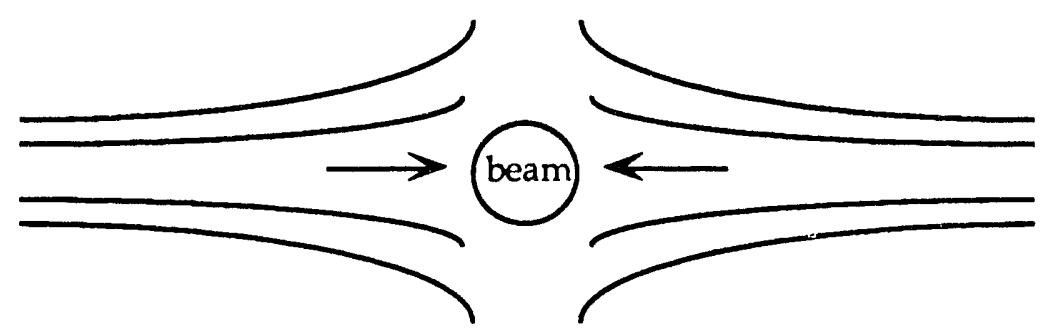

Figure IV.7

One-Dimensional Laser Trap

Atoms attempting to move laterally out of the atomic beam (directed into the page) are slowed by diverging laser beams tuned slightly below resonance. Four such diverging beams can be used (Balykin et al., 1988) to collimate a beam of sodium atoms in two dimensions, and six such beams can be used to make "optical molasses" in which atom motion is so strongly damped as to be diffusive. ${ }^{11}$ Sodium atoms have been loaded into optical molasses with densities up to $10^{8} \mathrm{~cm}^{-3}$ and diffusive escape times over 0.5 seconds. ${ }^{12}$ Cooling to energies equivalent to temperatures of tens of microKelvins, lower than the single photon momentum recoil limit, has recently been achieved.13 A US group ${ }^{14}$ has succeeded in extracting very slow sodium atoms from optical molasses to form an atomic "fountain," as first proposed by Zacharias in 1954. Narrow RF resonance fringes with $2-\mathrm{Hz}$ widths were observed, corresponding to the time for the very slow atoms to rise through an RF field region, be turned around, and fall back through the $R F$ field. These results have significant implications for future alkali microwave standards.

The second class of force is due to the electric dipole moment induced in an atom by laser light near resonance. As with resonance in a mechanical system,

11 S. Chu, L. Hollberg, J. E. Bjorkholm, A. Cable, and A. Ashkin, "Three-Dimensional Viscous Confinement and Cooling of Atoms by Resonance Radiation Pressure," Phys. Rev. Lett., 55 (1985), 48-51.

12 W. D. Phillips, P. L. Gould, and P. D. Lett, "Cooling, Stopping, and Trapping Atoms," Science, (1988), 877-883.

13 P. D. Lett, C. I. Westbrook, R. N. Watts, S. L. Rolston, P. L. Gould, H. J. Metcalf, and W. D. Phillips, "Atoms Laser-Cooled Below the Doppler-Cooling Limit," Proc. 4th Symp. on Frequency Standards and Metrology, Berlin: Springer-Verlag, 1989, 264-269.

14 M. A. Kasevich, E. Riis, S. Chu, and R. G. DeVoe, Digest of Conference on Quantum Electronics and Laser Science, Optical Society of America, Washington, DC, 1989. 
the induced dipole moment is in phase with the laser light if the light frequency is below resonance, and out of phase if the light frequency is above resonance. If there is a gradient in the laser-light intensity, the dipole force pulls atoms toward high light intensity when the light is below resonance, and expels atoms from the regions of high light intensity when the light frequency is above resonance. Using the dipole force to confine atoms was first proposed by V. S. Letokhov over 20 years ago (Letokhov, 1969). This was first realized at AT\&T Bell Laboratories in 1986 by focusing a laser beam tuned below resonance to an absolute maximum of intensity at the focus in the midst of optical molasses. ${ }^{15}$ Slow atoms fall from the optical molasses into the optical trap. A few hundred sodium atoms with energies up to the equivalent of $5-\mathrm{mK}$ temperatures can be held in a volume of $10^{-9} \mathrm{~cm}^{3}$ for roughly 1 second. It is also possible to make a trap using only radiation pressure ${ }^{16}$ to confine larger numbers of atoms $\left(10^{7}\right)$ in larger trapping volumes $\left(10^{-4} \mathrm{~cm}^{3}\right)$.

The spatially varying laser intensities in these optical traps shift the atom frequencies by position-dependent amounts which greatly complicates the prospects for precision spectroscopy and applications to frequency standards. Static traps that rely on the interaction of atomic magnetic dipole moments with spatially inhomogeneous magnetic fields can also trap neutral particles if their energies are low enough. The first such trap ${ }^{17}$ used oppositely directed magnetic field coils to produce a potential well having a volume of roughly $20 \mathrm{~cm}^{3}$, and was capable of holding sodium atoms with energies equivalent to temperatures less than $17 \mathrm{mK}$. A similar magnetic trap has been used to confine over $5 \times 10^{12}$ hydrogen atoms at densities near $10^{13} \mathrm{~cm}^{-3}$ for over 20 minutes at energies equivalent to $40 \mathrm{mK}$. In that case, the precooling was performed by collisions with superfluid liquid helium surfaces. The temperature can be lowered to as little as $3 \mathrm{mK}$ by decreasing the trapping field to allow the more energetic atoms to

15 S. Chu, J. E. Bjorkholm, A. Ashkin, and A. Cable, "Experimental Observation of Optically Trapped Atoms," Phys. Rev. Lett., 57(1986), 314-317.

16 E. L. Raab, M. Prentiss, A. E. Cablc, S. Chu, and D. E. Pritchard, "Trapping of Neutral Sodium Atoms with Radiation Pressure," Phys. Rev. Letl., 59 (1987), 2631-2634.

17 A. L. Migdall, J. V. Prodan, W. D. Phillips, T. H. Bergeman, and H. J. Metcalf, "First Observation of Magnetically Trapped Neutral Atoms," Phys. Rev. Lett., 54 (1985), 25962599. 
evaporate. ${ }^{18}$ Traps with very cold surfaces have the advantage of very low scattering out of the trap by unwanted background contamination.

Motion in the spatially varying magnetic fields of electrode traps perturbs the atom resonance frequencies and also complicates precision spectroscopy and frequency standard applications. However, further success in cooling trapped atoms may lead to confinement with very narrow resonance linewidths at sufficiently low velocities and in sufficiently small volumes to lead to frequency standard applications, as in the case of trapped ions.

\section{c. Discussion of Soviet Work}

Soviet physicists associated with V.S. Letokhov at the Spectroscopy Institute continue to play a leading role in the development of techniques to cool and trap neutrals using lasers. Letokhov himself made some of the earliest practical suggestions for manipulating atoms with laser light (Letokhov, 1969; Letokhov et al., 1976). Some of the first experimental cooling was performed by Letokhov and his colleagues (Balykin et al., 1980). (Their atomic beam collimation experiments were mentioned above.) In addition, this group has recently succeeded in specularly reflecting sodium atoms using the dipole force exerted by the very strong laser field intensity gradient in the evanescent light wave outside a quartz plate associated with total internal reflection within the plate (Balykin et al., 1988). As illustrated in Figure IV.8, specular reflection can occur when the Debroglie wavelength of the atom divided by the angle of incidence is greater than the surface roughness.

In this experiment, the angles were restricted by that condition to less than 0.007 radians because of the high thermal velocities of the atoms. However, laser cooled atoms could be specularly reflected at much larger angles, leading to a proposal by the Letokhov group for atom storage in which cold atoms circulate between two concave mirrors or around a ring in which they are reflected successively by mirrors (Balykin and Letokhov, 1989).

18 N. Mashuhara, J. M. Doyle, J. C. Sandberg, D. Kleppner, and T. J. Greytak, "Evaporative Cooling of Spin-Polarized Atomic Yydrogen," Phys. Rev. Lett., 61 (1988), 935-938. 


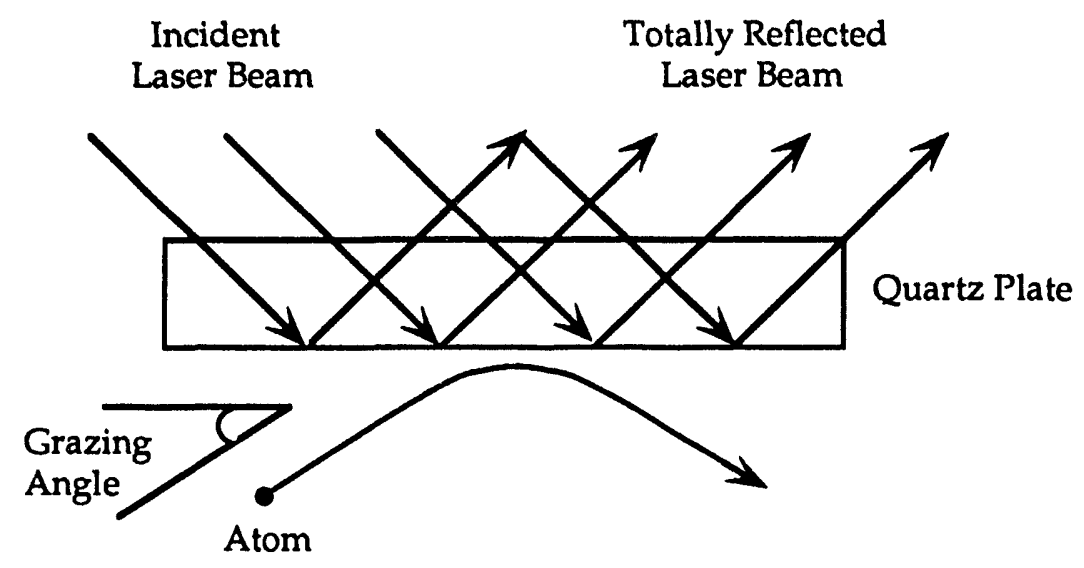

The light intensity just outside the quartz plate in which there is total reflection falls off with distance from the plate in a wavelength of the light, producing a very strong optical field gradient from which an atom is reflected specularly if its own wavelength divided by the grazing angle is greater than the surface roughness.

Figure IV.8

Specular Reflection of an Atom by a Strong Light Intensity Gradient

Recently, a group at the Lebedev Physics Institute succeeded in slowing a cesium atom beam to velocities equivalent to temperatures near $1^{\circ} \mathrm{K}$ using an external cavity injection laser (Akulshin et al., 1989a). This work is very significant, both because it represents the entry of a very important laboratory, and because it is directly applicable to cesium beam standards.

V. G. Minogin and colleagues in Letokhov's group have made significant contributions to the theory of laser cooling and trapping (Minogin, 1986; Minogin and Rozhdestvenskiy, 1987). At the Aussois Frequency Metrology Conference in 1981, Minogin with Letokhov made an interesting suggestion on the use of laser cooling to improve the performance of optical frequency standards, but there does not appear to have been any follow-up experimental work (Letokhov and Minogin, 1981). Recently, a team at the Ioffe Physical-Technical Institute in Leningrad made an interesting suggestion regarding a new transverse cooling effect that might be used to collimate atomic beams over large distances using a uniform coaxial laser beam (Amus'ya and Baltenkov, 1988). 
A special issue of the Journal of the Optical Society reviewed recent work in laser cooling and trapping of atoms with 28 invited papers (13 were from the United States, five from (West) Germany, four from the Soviet Union, three from France, and one each from Denmark, The Netherlands, and Italy). There was one experimental paper by Balykin et al. (1989) describing an extension of their previous studies on atomic beam collimation to localization of sodium atoms in a spherical standing wave. The experimental results confirmed their hypothesis that an appreciable fraction of the atoms are constrained by the dipole force to follow a spherical wavefront so long as the radius of curvature is large enough that the centrifugal force does not overpower the dipole force. These results suggest significant applications to bunching and guiding atomic beams for use, for example, in conventional cesium atomic beam frequency standards. The other three Soviet papers were theory papers. ${ }^{19}$ The overall proportion of Soviet theory papers to experimental papers is characteristic of their competitiveness in this field.

\section{d. Projections for the Future}

The Letokhov group is among the world leaders in the important new field of cooling and trapping neutrals using laser light. It is significant that physicists at the Lebedev Physics Institute have recently entered the field with an experiment to slow a cesium atomic beam, and that the Letokhov-Balykin group has concentrated on collimation techniques rather than on trapping. It is likely that slowing and collimating atomic beams for use in microwave standards will be the first significant application of laser light forces to frequency standards. Significant Soviet work can be expected in this area. Applications of cooling and trapping for improving optical frequency standards should also be expected in the near future. There is no indication of Soviet work on atomic hydrogen, which requires more sophisticated lasers, nor is there any indication of using electrode traps in conjunction with laser cooling or other cooling techniques. Such experiments might indicate a Soviet interest in entering the important contemporary field of trapped ion standards and the eventual use of trapped neutrals for precision spectroscopy and frequency standard applications.

Kazantsev and Krasnov, 1989; Minogin et al., 1989; Kazantsev et al., 1989. 


\section{Cryogenic Hydrogen Masers}

\section{a. Summary}

Cryogenic atomic hydrogen masers have been developed recently in the United States and Canada as a byproduct of cryogenic research on spin-polarized atomic hydrogen gas. Soviet theoreticians led by Yu. Kagan at the Kurchatov Atomic Energy Institute in Moscow have made significant contributions to the theory of collective effects in spin-polarized atomic hydrogen, but there is no evidence of experimental work in this field, or in the development of cryogenic hydrogen masers.

\section{b. Introduction}

Spin-polarized atomic hydrogen research was stimulated by the suggestion that hydrogen atoms with their electron spins aligned would interact so weakly as to undergo a transition to a macroscopic quantum state in the gas phase at sufficiently low temperatures and high densities. ${ }^{20}$ Almost as soon as any appreciable amount of hydrogen atom gas had been cooled to liquid helium temperatures, it was pointed out by several authors ${ }^{21}$ that cold hydrogen atom gas offered the following potential advantages in the operation of hydrogen maser frequency standards: (i) the spin exchange collision cross section, which limits the line $Q$ and radiated power in conventional hydrogen masers, decreases dramatically at low temperatures; (ii) the thermal noise, which requires the use of very long averaging times to achieve optimal stability in convention hydrogen masers, is proportionately less; and (iii) the molecular solid or superfluid liquid helium surfaces used in cryogenic hydrogen masers may produce more reproducible wall shifts. To date, one laboratory has developed a cryogenic maser using solid neon

21 S. B. Crampton, W. D. Phillips, and D. Kleppner, "Proposed Low Temperature Hydrogen Maser," Bull. Am. Phys. Soc., 23 (1978), 86.

R. F. C. Vessot, M. W. Levine, and E. M. Mattison, Proc. 9th Annual Precise Time and Time Interval Conf., 1978 (NASA Technical Memorandum No. 78104, 1978, 549 [unpublished]).

A. J. Berlinskiy and W. N. Hardy, "Cryogenic Masers," Proc. 13th Annual Precise Time and Time Interval (PTTI) Applications and Planning Mtg., 1981 (NASA Conference Publication 2220, 1981, 547-559). 
storage surfaces at roughly $10^{\circ} \mathrm{K}, 22$ and three laboratories have developed cryogenic masers using superfluid liquid helium surfaces at roughly $0.5^{\circ} \mathrm{K} . .^{23}$ Frequency shifts due to collisions between hydrogen atoms and the surface material either on the surface, or in the vapor phase above it, are illustrated in Figure IV.9 for liquid helium surfaces. ${ }^{24}$

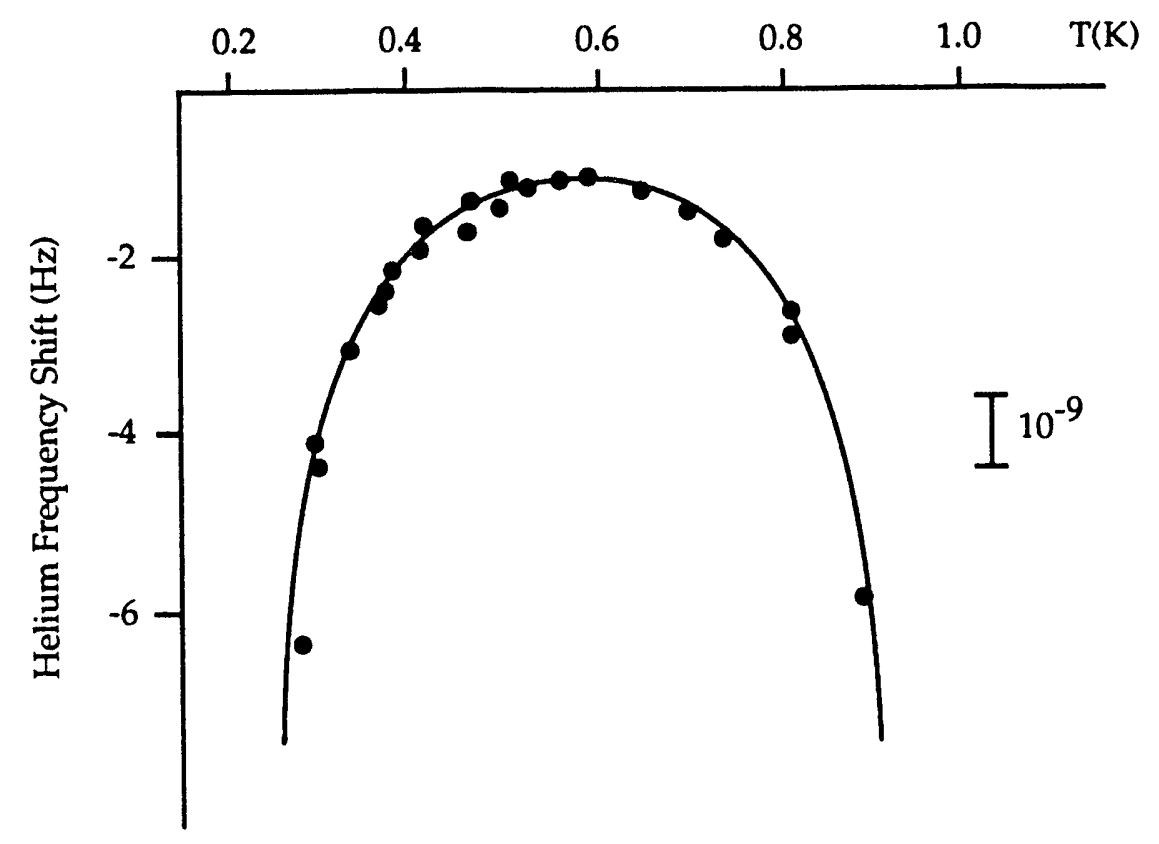

Figure IV.9

Hydrogen Frequency Shifts Due to Helium Collisions

22 S. B. Crampton, K. M. Jones, G. Nunes, and S. P. Souza, "Hydrogen Maser Oscillation at $10^{\circ}$ K," Proc. 16th Precise Time and Time Interval (PTTI) Applications and Planning Mtg., Greenbelt, Maryland, 1985 (NASA Technical Memorandum No. 8756, 1985, 339-349 [unpublished]).

23 H. F. Hess, G. P. Kochanski, J. M. Doyle, T. J. Greytak, and D. Kleppner, "Spin-Polarized Hydrogen Maser," Phys. Rev., A34 (1986), 1602-1604.

R. L. Walsworth, I. F. Silvera, H. P. Godfried, C. C. Agosta, R. F. C. Vessot, and E. M. Mattison, Phys. Rev. Lett., A34 (1986), 2550.

M. D. Hürliman, W. N. Hardy, A. J. Berlinskiy, and R. W. Cline, "Recirculating Cryogenic Hydrogen Maser," Phys. Rev., A34 (1986), 1605-1607.

24 M. Morrow, R. Jochemsen, A. J. Berlinsky, and W. N. Hardy, "Zero Field Hyperfine Resonance of Atomic Hydrogen for $0.18 \leq \mathrm{T} \leq 1 \mathrm{~K}$ : The Binding Energy of $\mathrm{H}$ on Liquid ${ }^{4} \mathrm{He}, "$ Phys. Rev. Lett., 46 (1981), 195; and Phys. Rev. Lett., 47 (1981), 547. 
For both helium and neon, there is a temperature at which the combination of wall collision and gas collision frequency shifts are first-order independent of temperature. This temperature depends on the ratio of storage chamber volume to surface area. The neon minimum comes at a temperature a factor of 20 higher than the helium shifts, and the neon shifts are an order of magnitude larger than the liquid helium shifts, which are themselves an order of magnitude larger than shifts due to the teflon in conventional hydrogen maser standards. The degree to which the relatively large shifts in cryogenic masers can be controlled and the potential short- and long-term frequency stabilities of such devices are currently under investigation in two US and one Canadian laboratory.

\section{c. Discussion of Soviet Work}

Soviet theorists have made significant contributions to knowledge of the behavior of polarized hydrogen atom gas at very low temperatures. In particular, Yu. Kagan and colleagues at the Kurchatov Atomic Energy Institute conducted the first successful analysis of the processes that led to decay of polarization and loss of atoms at low temperatures (Kagan et al., 1981). They successfully accounted for the observed spin decay due to binary collisions. They convincingly demonstrated that three-body recombination would limit achievable atom densities to values well below those required for Bose condensation at temperatures high enough to prevent condensation of the gas onto the storage chamber surfaces. Having shown that interactions of the atoms with the storage chamber surfaces would inevitably be important in the quest for the Bose condensation, Kagan et al. (1982) investigated the possible decrease of the accommodation coefficient at very low temperatures and studied the properties of the two-dimensional gas of atoms adsorbed on surfaces. Kagan and his colleagues have continued this work in many interesting papers dealing with spin-polarized atomic hydrogen, but there were no papers dealing specifically with applications to cryogenic hydrogen masers. Lectures by Dr. Kagan at Harvard University in 1989 on these subjects were very well received. There is no evidence of experimental Soviet work on spin-polarized atomic hydrogen or its applications to cryogenic hydrogen maser frequency standards, perhaps due to a scarcity of ${ }^{3} \mathrm{He}$ refrigerators and ${ }^{3} \mathrm{He}-{ }^{4} \mathrm{He}$ dilution refrigerators. 


\section{d. Projections for the Future}

Despite the promise of improved short-term stability for cryogenic hydrogen maser standards, and despite the Soviet interest in conventional hydrogen maser standards, there appears to be no Soviet work in this relatively new area. A collaboration between low-temperature physicists and atomic physicists investigating fundamental properties of spin-polarized atomic hydrogen, or a combination of cryogenic techniques with electrode trapping of neutrals might indicate a Soviet interest in developing cryogenic standards like the cryogenic hydrogen maser.

\section{CRYOGENIC ELECTROMECHANICAL OSCILLATORS}

\section{Summary}

Very stable electromechanical oscillators, such as the piezoelectric quartz crystal oscillators discussed in Chapter IX, are vitally important to many aspects of precision frequency measurement and time transfer, especially in their use in frequency synthesis chains and as local oscillators. Much better electromechanical oscillators are needed for these purposes, and to serve as flywheel standards to transfer to reasonable measurement times the excellent long-term stability of trapped particle standards. Cryogenic dielectric resonators are likely to play a leading role. Soviet scientists have contributed some of the most important ideas and experiments, even though their materials are not as good as those available in the West.

Soviet work in the area of cryogenic electromechanical oscillators demonstrates the following characteristics:

- excellent theoretical studies of superconducting cavity and dielectric resonators;

- excellent studies of measuremen: theory and fundamental limitations imposed by thermal and quantum effects; and

- excellent experimental work with dielectric resonators. 


\section{Introduction}

The fundamental limit to the frequency stability of a classical, self-excited oscillator is

$$
\frac{\Delta v}{v}=\frac{1}{\mathrm{Q}} \sqrt{\frac{\mathrm{kT}}{2 \mathrm{P} \tau}}
$$

where $\mathrm{T}$ is the absolute temperature, $\mathrm{P}$ is the power delivered, $\tau$ is the averaging time, and $1 / Q$ is the sum of all the dissipative losses. ${ }^{25}$ At very low temperatures, such that $k T<h v / 2$, there is a fundamental quantum mechanical floor to the fluctuations ${ }^{26}$

$$
\frac{\Delta v}{v}=\frac{1}{\mathrm{Q}} \sqrt{\frac{\mathrm{hv}}{4 \mathrm{P} \tau}} \approx \frac{2 \times 10^{-20}}{\sqrt{\tau}} .
$$

Cryogenic techniques help by reducing the thermal noise toward the quantum mechanical limit, and by freezing out dissipation mechanisms that reduce $Q$. Dissipation is linked not only to frequency fluctuations, but also to nonlinearities which cause power-dependent frequency shifts. Consequently, understanding dissipation mechanisms is very important, and high $Q$ is essential to performance. Dimensional stability, which also improves at low temperatures, is also an important consideration because the frequencies of electromechanical resonators are determined directly by their physical dimensions. The fractional frequency shift due to a change of dimension roughly equals the fractional dimension change, so that stability to a few parts in $10^{15}$ requires dimensional stability to a few parts in $10^{15}$, which, even for reasonably large resonators, is less than nuclear dimensions.

25 W. A. Edson, "Noise in Oscillators," Proc. IRE, 48 (1960), 1454-1456.

26 W. E. Lamb, Quantum Optics and Electronics, eds., C. DeWitt, A. Blandin, and C. CohenTannoudji, New York: Gordon \& Breach, 1965, 331-381. 
Hollow superconducting microwave cavities with $Q$ as high as $10^{11}$ have been developed 27 and used as the basis for oscillators achieving frequency stabilities to a few parts in $10^{16}$ at $\tau=10$ seconds. ${ }^{28}$ No significant improvements have since been made, perhaps because the mechanical stability requirements are severe. Power level changes produce variable mechanical stresses; these also affect the $Q$ through surface heating changes. Although coefficients of thermal expansion are small at low temperatures, the temperature must be controlled to the microKelvin level at $2^{\circ} \mathrm{K}$ for a niobium resonator to hold its frequency to a few parts in $10^{15}$.

These mechanical problems are considerably reduced by making the interior of the resonator a suitable dielectric crystal coated with a superconducting film. With this technique, the surface coating is protected at the dielectric interface, and the structural rigidity of the interior material greatly benefits the mechanical stability of the resonator. Cavity strain due to pressure fluctuations from variations in the stored energy is greatly reduced. Resonators of this type have been built and tested using sapphire, which is the best known dielectric material having a loss tangent (contribution to $1 / \mathrm{Q}$ ) less than $10^{-9}$ at low temperatures. Soviet scientists have been successful in their study of such resonators and have made many contributions to theoretical and experimental advances in this area. The dominant instability in these resonators is the effect of changes of skin depth due to power fluctuations. For fractional frequency stability at $3 \mathrm{GHz}$ of the order of 1 in $10^{14}$ at $1.6^{\circ} \mathrm{K}$, the temperature must be held to within $3 \mathrm{mK}$, and the power must be held constant within $0.3 \mathrm{~mW}$.

Very high $Q$ electromagnetic resonators can be made without conductive coatings by using total internal reflection at the inside of dielectric surfaces. Evoking their acoustical analogs, these are described as "whispering-gallery" resonators (Braginskiy et al., 1989). At room temperatures, these can have $Q$ values of a few tens of thousands. Since there are no temperature-dependent skin effects, their temperature coefficient of fractional frequency variation is close to the coefficient of linear expansion, which is roughly $5 \times 10^{-12} / \mathrm{K}$ at $2^{\circ} \mathrm{K}$. There are

27

28

J. P. Turneaure, Proc. IEEE Conf. on Applied Superconductivity, New York, 1972, 154.

S. R. Steia, "Application of Superconductivity io Precision Osciliators," Proc. 29th Symp. on Frequency Control, 1975, 321-329. 
radiative losses caused by the curvature of the internally reflecting surfaces, however, by choosing a high-order mode (where there are as many as 20 reflections about the circumference), and $R / \lambda=1.4$, where $R$ is the radius and $\lambda$ is the vacuum wavelength, the calculated $Q$ is greater than $6 \times 10^{11}$. However, there are other loss mechanisms associated with the homogeneity of the dielectric constant throughout the volume of the ring and losses both from surface roughness scattering and from a lack of perfect symmetry.

\section{Discussion of Soviet Work}

A paper by V. M. Pudalov (1980) provides a complete evaluation of the use of superccnducting microwave resonators in metrology, and indicates an excellent understanding of the state of the art in the frequency stability of rubidium, cesium, and hydrogen standards in relation to that of superconducting oscillators, mostly developed in the United States. The author points out the potential advantages (tunability, high power, very good spectral purity, and good mediumterm stability) of such oscillators for flywheels in atomic standards and infrared frequency stabilized lasers. Various techniques for supplying regeneration to superconducting resonators are discussed, including the possibility of using a microstrip version of the resonator. It is clear from this paper and its references that the potential benefits of superconducting resonators and oscillators which used them were well understood and appreciated and that there was ongoing work on these devices in the Soviet Union. Since the best results quoted were US results (such as those of Stein and Turneaure), it appears that Soviet materials and materials processing may have lagged those of the United States.

Extremely low losses for electromagnetic waves in single-crystal dielectrics were discussed in a short paper by Professor V. B. Braginskiy and his colleagues at Moscow State University (Bagdasarov et al., 1984). Their previous measurements of resonance quality factors of up to $4 \times 10^{8}$ were reported, as subsequently confirmed by a US measurement. ${ }^{29}$ Their measurements of the loss tangent of the sapphire closely followed the $\mathrm{T}^{5}$ law predicted by V. L. Gurevich (1979) on the basis of lattice anharmonicity in hexagonal crystals down to $60^{\circ} \mathrm{K}$. Two flattening regions occur at lower temperatures with $\mathrm{Q} \approx 10^{8}$ for $25^{\circ} \mathrm{K}<\mathrm{T}<60^{\circ} \mathrm{K}$ and $4 \times 10^{8}$ 
for $3.5^{\circ} \mathrm{K}<\mathrm{T}<10^{\circ} \mathrm{K}$. The limits to $\mathrm{Q}$ were attributed to defects and impurities in the crystals available at that time.

Quantum considerations in very low loss systems have been studied extensively by Professor Braginskiy, who was one of the first to recognize that, in principle, it is possible to circumvent the limits imposed by the Heisenberg uncertainty relation by "squeezing" the limits on one of a pair of complementary variables at the expense of the other. Braginskiy's book, entitled Systems with Small Dissipation, is probably the best treatise on high $\mathrm{Q}$ resonators available, and describes both the experimental work then in progress in the Soviet Union and other work reported worldwide (Braginskiy et al., 1985). The highest $Q$ mechanical resonator was made from a single-crystal sapphire that had $Q \approx 4 \times 10^{9}$ at temperature $4^{\circ} \mathrm{K}$ and a frequency of $38 \mathrm{kHz}$. Produced at Moscow State University, this device represented the state of the art at that time.

Frequency drifts of potassium tantalate dielectric resonators were studied by Belokopytov and colleagues (Belokopytov et al., 1985). Time constants on the order of $10^{4}$ seconds were attributed to changes of the dielectric constant near the surface induced by large electric fields caused by band bending.

A theoretical and experimental study of the mode spectrum, temperature coefficient, and $Q$ values for sapphire ring resonators was conducted by Bun'kov et al. (1987). In this paper, it was shown that radiation losses cannot account for the limiting $Q$ values observed for high-order azimuthal modes, therefore, they must be dependent on the quality of the material and the fabrication of the resonator. The frequency temperature coefficient at low temperatures was shown to be due to the permittivity, rather than to the dimensional coefficient. The frequency passed through an extremum at roughly $13^{\circ} \mathrm{K}$. This feature of a frequency extremum apparently occurs at different temperatures in different crystals.

An update on the status of cryogenic oscillators (Braginskiy, 1988) lists $Q$ values for single-crystal sapphire mechanical resonators at $4^{\circ} \mathrm{K}$ as $5 \times 10^{9}$ at frequencies of $30 \mathrm{kHz}$, and for superconducting niobium resonators at $1.3^{\circ} \mathrm{K}$ as $5 \times 10^{11}$ at $10 \mathrm{GHz}$. Soviet work on whispering-gallery resonators at $10 \mathrm{GHz}$ has produced $\mathrm{Q}$ levels of $1.3 \times 10^{9}$ and it appears that they have found a convenient extremum in 
the temperature-versus-resonance frequency relation for their sapphire near $9^{\circ} \mathrm{K}$, where $\delta \mathrm{f} / \mathrm{f}=1 \times 10^{-9}\left(\Delta \mathrm{T}^{2}\right)$ which will allow frequency drift control at the $10^{-17}$ to 10-18 level with easily achieved temperature control at the $10^{-4} \mathrm{~K}$ level. The extremum is believed to result from a very small level of residual chromium impurity in the sapphire. Lower levels of impurity are expected to bring the operating point to a lower temperature where even higher $Q$ is expected. The best results for dielectric resonators quoted in this paper are those of the US Jet Propulsion Laboratory. 30

In a recent study of whispering-gallery microresonators at near-infrared wavelengths, Braginskiy et al. (1989) point out that optical bistability of these small (100- $\mu \mathrm{m}$ diameter) microresonators could make them useful in optical computers.

There is no evidence of Soviet use of high $\mathrm{T}_{c}$ superconductors as resonators, although there is some work in this area in the United States.

\section{Projections for the Future}

The Soviet Union is at the leading edge of theory in cryogenic electromechanical oscillators. There is significant experimental development of frequency standard applications at Moscow State University. Soviet competitiveness with the United States will likely depend on the availability of very high purity materials, an area in which the Soviet Union appears to be lagging. Soviet improvements of $Q$ and the development of a working frequency standard exploiting the frequency shift extremum in sapphire ring resonators near $10^{\circ} \mathrm{K}$ or below would be landmark achievements.

\section{E. KEY SOVIET RESEARCH PERSONNEL AND FACILITIES}

A list of key Soviet research personnel and facilities involved in new and emerging frequency standard technologies is presented in Table IV.1.

30 S. Thakoor, D. M. Strayer, G. J. Dick, and J. E. Mercereau, "A Lead-on-Sapphire Superconducting Cavity of Superior Quality," J. Appl. Phiys., 59, 3(1986), 854-858. 


\section{Table IV.1 \\ KEY SOVIET RESEARCH PERSONNEL AND FACILITIES- NEW AND EMERGING FREQUENCY STANDARD TECHNOLOGIES}

\section{OPTICALLY PUMPED MICROWAVE STANDARDS}

Engineering Physics Institute, Moscow

L. A. Budkin

V. K. Yegorov

V. A. Maslov

Leningrad Polytechnic Institute im. M. I. Kalinin, Leningrad

M. B. Gornyy

\section{SATURATED ABSORPTION STABILIZED LASERS}

Physics Institute im. P. N. Lebedev, AS USSR, Moscow

N. G. Basov

M. A. Gubin

Thermal Physics Institute, Siberian Branch AS USSR, Novosibirsk

S. N. Bagayev

V. P. Chebotayev

Physical-Technical and Radio Engineering Measurements All-Union Scientific Research Institute, Mendeleyevo (Moscow)

Yu. S. Domnin

\section{TRAPPED ION (AND RELATED) THEORY}

Thermal Physics Institute, Siberian Branch AS USSR, Novosibirsk

Ye. V. Baklanov

Ye. A. Titov 


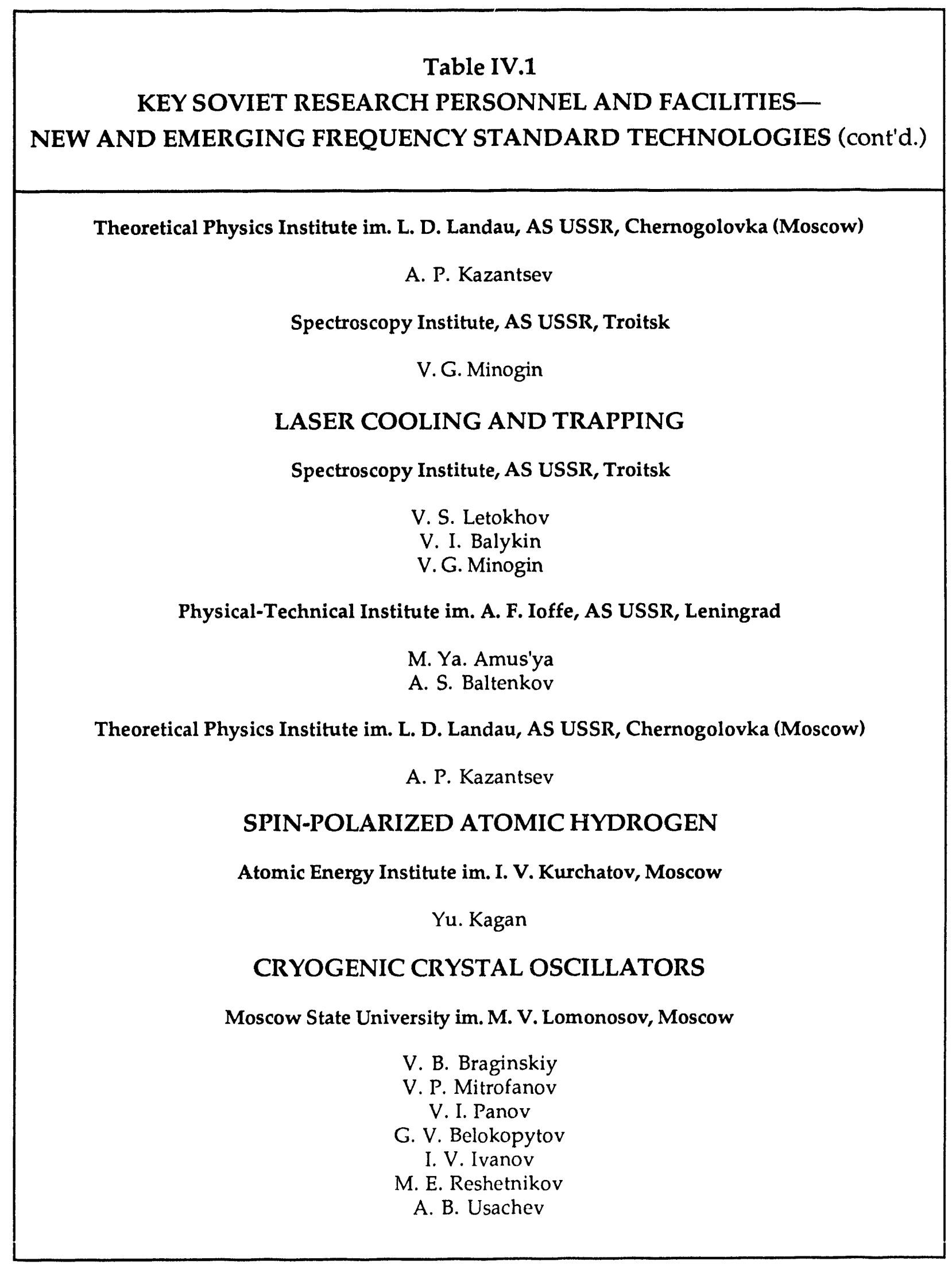




\section{Table IV.1}

KEY SOVIET RESEARCH PERSONNEL AND FACILITIES-

NEW AND EMERGING FREQUENCY STANDARD TECHNOLOGIES (cont'd.)

Physics Institute im. P. N. Lebedev, AS USSR, Moscow

A. M. Akulshin

V. L. Velichanskiy

R. G. Gamidov

V. I. Malakhova

V. V. Nikitin

V. A. Sautenkov

M. Sh. Kobyakova

G. T. Pak 


\section{CHAPTER IV: NEW AND EMERGING FREQUENCY STANDARD TECHNOLOGIES REFERENCES}

Akulshin, A. M., V. L. Velichanskiy (Velichanskii), R. G. Gamidov, V. I. Malakhova, V. V. Nikitin, V. A. Sautenkov, M. Sh. Kobyakova (Kobiakova), and G. T. Pak, "Formation of a Narrow Velocity Distribution of an Atomic Cesium Beam by the Resonant Radiation of an Injector Laser," Sov. J. Quantum Electron., 7(1989a), 1507-1510.

Akulshin, A. M., V. L. Velichanskiy (Velichanskii), A. S. Zibrov, V. V. Nikitin, V. A. Sautenkov, and G. G. Kharisov, "Stabilization of the Frequency of a Highly Coherent Injection Laser Using Intra-Doppler Saturated Absorption Resonances," Sov. J. Quantum Electron., 18, 10(1988), 1214-1215.

Akulshin, A. M., V. V. Nikitin, V. A. Sautenkov, V. L. Velichanskiy (Velichanskii), E. K. Yurkin (Iurkin), and A. S. Zibrov, "Frequency Stabilization of Highly Coherent AlGaAs Diode Lasers," Proc. IV Symp. on Frequency Standards and Metrology, Berlin: Springer-Verlag, 1989b, 236-241.

Amus'ya (Amusia), M. Ya., and A. S. Baltenkov, "Radiation Cooling of Atoms in a Uniform Optical Field," Sov. Tech. Phys. Lett., 14, 5(1988), 388-389.

Bagayev (Bagaev), S. N., A. E. Baklanov, V. P. Chebotayev (Chebotaev), and A. S. Dychkov, "Superhigh Resolution Spectroscopy in Methane with Cold Molecules," Revue Romaine de Physique, 33, 4-6(1988), 361-367.

Bagayev (Bagaev), S. N., and V. P. Chebotayev (Chebotaev), "Laser Frequency Standards," Sov. Phys.-Usp., 29, 1(1986), 82-103.

Bagayev (Bagaev), S. N., V. P. Chebotayev (Chebotaev), A. K. Dmitriyev (Dmitriev), A. E. Om, Y. V. Nekrasov, and B. N. Skvortsov, "Obtaining of Supernarrow Saturated Absorption Resonances of Cold Particles and Their Use for Optical Frequency Standards," Proc. IV Symp. on Frequency Standards and Metrology, Berlin: Springer-Verlag, 1989a, 277-281.

Bagayev (Bagaev), S. N., V. P. Chebotayev (Chebotaev), A. S. Dychkov, and S. V. Malt'sev, "Supernarrow Resonances in Methane on E-line of the $P(7)$ Transition of the $v_{3}$ Band and Their Application in Optical Frequency Standards," J. Physique (Paris), 42 (1981), C8-21-28.

Bagayev (Bagaev), S. N., V. P. Chebotayev (Chebotaev), V. M. Klement'yev (Klement'ev, Klementiev), B. A. Timchenko, and V. F. Zakharash, "Direct Comparison of the Characteristics of Optical and Hydrogen Frequency Standards," Proc. IV Symp. on Frequency Standards and Metrology, Berlin: Springer-Verlag, 1989b, 191-194.

Bagdasarov, Kh. S., V. B. Braginskiy (Braginskii), V. I. Panov, and V. S. Il'chenko, "Anomalously Low Dissipation of Electromagnetic Waves in Perfect Single-Crystal Dielectrics," Sov. Phys.-Usp, $28,1(1985), 95-96$.

Baklanov, Ye. V., and V. P. Chebotayev (Chebotaev), "Resonant Light Absorption by the Ordered Structures of Ions Stored in a Trap," Appl. Phys. B, 39 (1986), 179-181.

Baklanov, Ye. V., V. P. Chebotayev (Chebotaev), and Ye. A. Titov, "A Lineshape of the Trapped Particle in Anharmonic Potential," Appl. Phys., 20, 4(1979), 361-363. 
Baklanov, Ye. V., V. P. Chebotayev (Chebotaev), and Ye. A. Titov, "Optical Resonances of Trapped Particles," Appl. Phys., 25 (1981), 81-86.

Balykin, V. I., and V. S. Letokhov, "Laser Optics of Neutral Atomic Beams," Physics Today, 42, 4(1989), 23-28.

Balykin, V. I., V. S. Letokhov, and V. I. Mishin, "Cooling of Sodium Atoms by Resonant Laser Emission," Sov. Phys.-JETP, 51, 4(1980), 692-696.

Balykin, V. I., V. S. Letokhov, Yu. B. Ovchinnikov, and A. I. Sidorov, "Focusing of an Atomic Beam and Imaging of Atomic Sources by Means of a Laser Lens Based on Resonance-Radiation Pressure," J. Modern Optics, 35, 1(1988), 17-34.

Balykin, V. I., Yu. E. Lozovik, Yu. B. Ovchinnikov, A. I. Sidorov, S. V. Shul'ga, and V. S. Letokhov, "One-Dimensional Localization of Atoms in a Standing Spherical Light Wave," J. Opt. Soc. Am. B, $6,11(1989), 2178-2187$.

Basov, N. G., E. M. Belenov, M. I. Volknov, M. A. Gubin, V. V. Nikitin, and V. N. Troshagin, "Frequency Stabilization of a Ring Laser," Sov. Phys.JETP., 15 (1972), 659-661.

Basov, N. G., M. A. Gubin, V. V. Nikitin, A. V. Nikul'chin, E. D. Protsenko, D. A. Tyurikov (Tiurikov), and A. S. Shelkovnikov, "Transportable Optical Frequency Standard and Results of Its Metrological Tests," Sov. J. Quantum Electron., 17, 4(1987), 545-547.

Basov, N. G., M. A. Gubin, V. V. Nikitin, A. V. Nikul'chin, D. A. Tyurikov (Tiurikov), V. N. Petrovskiy (Petrovskii), and E. D. Protsenko, "A Highly Sensitive Technique of Detecting Supernarrow Spectral Lines Based on the Frequency Resonances of a Double-Mode Laser," $J$. Physique (Paris), 42, 12(1981), C8-89-95.

Belokopytov, G. V., I. V. Ivanov, M. E. Reshetnikov, and A. B. Usachev, "Slow Relaxation Processes in Cryogenic Dielectric $\mathrm{KTaO}_{3}$ Resonators," Sov. Phys.-Tech. Phys., 30, 10(1985), 1229-1230.

Braginskiy (Braginskii), V. B., M. L. Gorodetskiy (Gorodetskii), and V. S. Il'chenko, "Quality Factor and Non-Linear Properties of Optical Whispering-Gallery Modes," Phys. Lett. A, 137, 7-8(1989), 393-397.

Braginskiy (Braginskii), V. B., "Resolution in Macroscopic Measurements: Progress and Prospects," Sov. Phys.-Usp., 31, 9(1988), 836-849.

Braginskiy (Braginskii), V. B., V. P. Mitrofanov, and V. I. Panov, Systems with Small Dissipation, University of Chicago Press, 1985.

Budkin, L. A., A. I. Pikhtelev, S. L. Puzanov, and B. P. Fateyev (Fateev), "Laser-Pumped Quantum Discriminator," Radio Eng. Electron. Phys., 29, 6(1984c), 122-126.

Budkin, L. A., M. N. Penenkov, A. I. Pikhtelev, and S. L. Puzanov, "Light Shifts of the Frequency of a Quantum Discriminator with Laser Pumping," Radiophys. Quantum Electron., 27, 6(1984b), 479482.

Budkin, L. A., M. N. Penenkov, and A. I. Pikhtelev, "Problem of Double Resonance in a Three-Level System," Opt. Spectrosc., 59, 5(1985), 588-590. 
Budkin, L. A., O. G. Okhotnikov, G. T. Pak, A. I. Pikhtelev, and S. L. Puzanov, "A Source of Optical Pumping for High-Resolution Spectroscopy Based on a Semiconductor Laser," J. Appl. Spectrosc., 40, 1(1984a), 12-122.

Budkin, L. A., V. N. Gutsaki, V. I. Dudkin, A. A. Lyalyaskin (Lialiaskin), V. Yu. Petrun'kin, A. I. Pikhtelev, and V. V. Semenov, "Double Resonance in an Alkali Medium with Laser Optical Pumping," Sov. J. Comm. Technol., 33, 5(1988), 177-179.

Bun'kov, S. N., B. A. Vytorushin, V. N. Yegorov (Egorov), V. I. Konstantinov, V. L. Masalov, and P. V. Smirnov, "Cooled Dielectric Resonators for Frequency Stabilization," Sov. J. Commun. Technol. Electron., 32, 9(1987), 165-173.

Chebotayev (Chebotaev), V. P., "Optical Time Scale," J. Physique (Paris), 42, 12(1981), C8-505512.

Chebotayev (Chebotaev), V. P., "Use of Ultranarrow Resonance in Spectroscopy and for Stabilizing Gas Laser Frequencies," Sov. J. Quantum Electron., 8, 9(1978), 1130-1135.

Domnin, Yu. S., N. B. Koshelyayevskiy (Kosheliaevskii), A. N. Malimon, V. M. Tatarenkov, and P. S. Shumyatskiy (Shumiatskii), "Infrared Frequency Standard Based on Osmium Tetraoxide," Sov. J. Quantum Electron., 17, 6(1987), 801-803.

Domnin, Yu. S., V. M. Tatarenkov, and P. S. Shumyatskiy (Shumiatskii), "Absolute Frequency Measurements of Lasers in Submillimeter Infrared Range," Meas. Tech., 19, 10(1976), 1475-1479.

Gornyy (Gornyi), M. B., N. A. Dovator, R. A. Zhitnikov, and B. G. Matisov, "Spatial Inversion of the Population Difference of Hyperfine Sublevels of the Ground State of Cesium Atoms During Optical Pumping," Sov. Tech. Phys. Lett., 10, 1(1984), 15-16.

Gubin, M. A., A. V. Nikulchin, and D. A. Tyurikov (Tiurikov), "Double Mode He-Ne/CH4 Laser Stabilized by Methane Resonances with Relative Width of 10-11," Proc. IV Symp. on Frequency Standards and Metrology, Berlin: Springer-Verlag, 1989, 206-211.

Gurevich, V. L., "Dielectric Losses in Crystals," Sov. Phys.-Solid State, 21, 11(1979), 1993-1998.

Kagan, Yu., G. V. Shlyapnikov (Shliapnikov), I. A. Vartanyants (Vartaniants), and N. A. Glukhov, "Quasi-Two-Dimensional Spin-Polarized Atomic Hydrogen," JETP Lett., 35, 9(1982), 477.

Kagan, Yu., I. A. Vartanyants (Vartaniants), and G. V. Shlyapnikov (Shliapnikov), "Kinetics of Decay of Metastable Gas Phase of Polarized Atomic Hydrogen at Low Temperatures," Sov. Phys.JETP, 54, 3(1981), 590-604.

Kazantsev, A. P., "Acceleration of Atoms by a Resonance Field," Sov. Phys.-JETP, 36 (1973), 861864 .

Kazantsev, A. P., and I. V. Krasnov, "Rectification Effect of a Radiation Force," J. Opt. Soc. Am. B, $6,11(1989), 2140-2148$.

Kazantsev, A. P., G. I. Surdutovich, D. O. Chudesnikov, and V. P. Yakolev, "Scattering, Velocity Bunching, and Self-Localization of Atoms in a Light Field," J. Opt. Soc. Am. B, 6, 11(1989), 21302139. 
Kazantsev, A. P., V. S. Smirnov, G. I. Surdutovich, D. O. Chudesnikov, and V. P. Yakovlev, "Kinetic Phenomena of Atomic Motion in a Light Field," J. Opt. Soc. Am. B, 2 (1985), 1731.

Kurochkin, V. Yu., V. N. Petrovskiy (Petrovskii), E. D. Protsenko, and A. N. Rurukin, "ComplexCavity Two-Mode $\mathrm{CO}_{2} / \mathrm{SF}_{6}$ Laser," Sov. J. Quantum Electron., 18, 6(1988), 781-782.

Letokhov, V. S., "Narrowing of the Doppler Effect in a Standing Light Wave," JETP Lett., 7 (1968), 272-275.

Letokhov, V. S., and V. G. Minogin, "Laser Cooling of Atoms and Its Application in Frequency Standards," J. Physique (Paris), 42 (1981), C8-347-355.

Letokhov, V. S., and V. G. Minogin, "Laser Radiation Pressure on Free Atoms," Phys. Rep., 73 (1981), 1 .

Letokhov, V. S., V. G. Minogin, and B. D. Pavlik, Opt. Comm., 19 (1976), 72.

Lisitsyn, V. N., and V. P. Chebotayev (Chebotaev), "Absorption Saturation Effects in a Gas Laser," Sov. Phys.-JETP, 27 (1968), 227-229.

Minogin, V. G., "Compression of Atomic Beams by Laser Radiation Pressure," Opt. Spectrosc., 60, 5(1986), 657-658.

Minogin, V. G., M. A. Olshany, and S. U. Shulga, "Laser Cooling of Atoms Below the Single-Photon Classical Limit," J. Opt. Soc. Am. B, 6, 11(1989), 2108-2111.

Minogin, V. G., and Yu. V. Rozhdestvenskiy (Rozhdestvenskii), J. Appl. Phys. B, 34 (1984), 161.

Minogin, V. G., and Yu. V. Rozhdestvenskiy (Rozhdestvenskii), "Stable Trapping of Atoms in the Field of Two Standing Light Waves," Opt. Spectrosc., 63, 2(1987), 138-139.

Pudalov, V. M., "Superconducting SHF Resonators and Their Use in Metrology," Meas. Tech., 23, 7(1980), 600-601.

Titov, Ye. A., and V. A. Ulybin, "Influence of Thermal Effects on the Two-Photon Absorption Line Profile of Ions Captured in a Trap," Sov. J. Quantum Electron., 12, 5(1982), 610-614.

Vyatchanin (Viatchanin), S. P., Dokl. AS USSR, 234 (1977), 1295.

Yegorov (Egorov), V. K., and V. A. Maslov, "Calculation of the Efficiency of Optical Pumping of ${ }^{133} \mathrm{Cs}$ by Monochromatic Radiation," Sov. J. Quantum Electron., 13, 12(1983), 1635-1636.

Zakharyash (Zakhariash), V. F., V. M. Klement'yev (Klement'ev, Klementiev), P. A. Losev, B. A. Timchenko, V. V. Yumin (Iumin), V. P. Chebotayev (Chebotaev), and S. N. Bagayev (Bagaev), "A Submillimeter Backward Wave Tube Frequency Locked to a $\mathrm{HCOOH}$ Laser for a Submillimeter Spectrometer," Sov. J. Quantum Electron., 89, 6(1989), 1513-1515.

\section{ADDITIONAL WORKS CONSULTED}

Alekseyev (Alekseev), V. A., "The Effects of Collisions in Precision Spectroscopy," I. Physique (Paris), 42, 12(1981), C8-29-35. 
Budkin, L. A., A. I. Pikhtelev, and S. L. Puzanov, "Radiooptical Double Resonance in Vapors of Alkali Metals," Radiophys. Quantum Electron., 26, 5(1983), 416-422.

Vtorushin, B. A., V. N. Yegorov (Egorov), and V. L. Maslov, "A Low-Noise Ultrahigh Frequency Generator Stabilized by a Dielectric Resonator," Instrum. Exp. Tech., 84, 3(1984), 236 (abstract only). 
(blank)

IV-44 


\section{CHAPTER V \\ TIME AND FREQUENCY TRANSFER AND DISSEMINATION}

\section{A. SUMMARY}

Soviet time and frequency dissemination techniques can be categorized into four areas: (i) satellite techniques, (ii) traditional terrestrial techniques, (iii) nontraditional techniques, and (iv) communication systems techniques.

The Soviet satellite technique, GLONASS, is very similar to the US Global Positioning System (GPS). GLONASS is planned to operate 24 orbiting satellites, each having atomic clocks on board. The Soviet Union is active in its use of television (TV) timing, using both satellites and terrestrial microwave means. As is true for the United States, the synchronization of Soviet communication systems via satellites and microwave terrestrial means is an important issue. The Soviet Union appears to have no equivalent to the US NOAA/GOES weather and time satellite pair, which basically serve the Americas and much of the Pacific and Atlantic oceans. Japan and Europe have an equivalent to GOES. The Soviet Union does not appear to have conducted any two-way satellite timetransfer experir.ents. ${ }^{1}$

The Soviet Union has most of the traditional terrestrial techniques which use the VLF, LF, MF, and HF standard time and frequency bands. In the case of the last two, the Soviet Union uses a broadcast frequency that is $4 \mathrm{kHz}$ off the approved frequency, which considerably reduces the interference with similar services being broadcast by other countries. Due to the lack of interference, the Soviet time and frequency broadcast services get more use than the others, as can be seen in Figure V.1 which is taken from an International Radio Consultarive Committee (CCIR) document. With the modulation present, this $4-\mathrm{kHz}$ offset probably causes Soviet transmitted frequencies to trickle over into the guard-

1 Bordovitsyna et al., 1985; Fedorov et al., 1973, 1987; Kholshevnikov et al., 1984; Paliy et al., 1972, 1985; '’aliy and Fedorov, 1972, 1975; Paliy, 1970.

F. Cordara, P. G. Galliano, V. Pettiti, S. Leschiutta, Jin-An Song, Heng-Qio Zheng, DingChang Lou, and Ven-Zhung Liu, "Synchronization Experiment Between the IFN (Italy) and the Shaanxi Observatory (China) Time Scales Via SIRIO 1 Satellite," Proc. 25th Symp. on Space Stations and Their Utilizations (sic), Rome, Italy, (26-28 March 1985), 85. 
band where no one is supposed to be transmitting. The Soviet Union uses TV timing techniques extensively, both on a terrestrial basis as well as by satellite. The United States has a much larger number of rubidium gas cell frequency standards than does the Soviet Union, but it appears that the Soviet Union does much more with rubidium gas cell portable clocks than does the United Statesboth for commercial uses and for defense. There are several other terrestrial techniques being developed in other countries. It is likely that the Soviet Union is also working on these techniques, but there was no evidence of this in the literature. Soviet researchers do not appear to be doing the type of work that is being conducted at the US Jet Propulsion Laboratory, in which optical fibers are used for ultra accurate time and frequency transfer over short distances. ${ }^{2}$

Non-traditional techniques used by the Soviet Union include relaying time signals off meteor trails. This technique was originally developed in the West, but the Soviet Union has exploited it and taken its accuracy further than any other country. One disadvantage of this technique is that the broadcast frequencies giving the best reflections are in the TV band, which causes interference with commercial TV at some frequency bands. To avoid this, Soviet scientists perform the time transfers during early morning hours. The United States is aware of this meteor trail technique but has not felt a need to develop it because it has other techniques, such as GPS, which are more cost effective and easier to use. Another non-traditional technique used by Soviet researchers is their hydrogen maser work. The Soviet Union has developed hydrogen masers for portable clocks and has accomplished more in this area than any other country. The Soviet Union is unique in its use of active hydrogen masers as portable clocks. ${ }^{3}$

Soviet scientists clearly have studied the US literature regarding communication systems and are conducting some interesting work in this area. Keeping the frequencies the same throughout a communications network does not require the delays to be calibrated. However, Soviet scientists sometimes appear to calibrate the delays in their microwave relay systems using reciprocity techniques. This would allow them to improve the efficiency of time-domain mul-

2 Bolotnikov and Polyak, 1976; Cherenkov, 1974; Fleer, 1972; Fleer and Stepanov, 1972; Fleer and Shichavelev, 1976; Vasin et al., 1972.

3

Dudnik et al., 1972, 1973, 1986; Matveyenko, 1987; Minullin et al., 1971. 
tiplexing of transmissions. Otherwise, Soviet synchronization of nodes appears to be very similar to that of the West (Barkov, 1985).

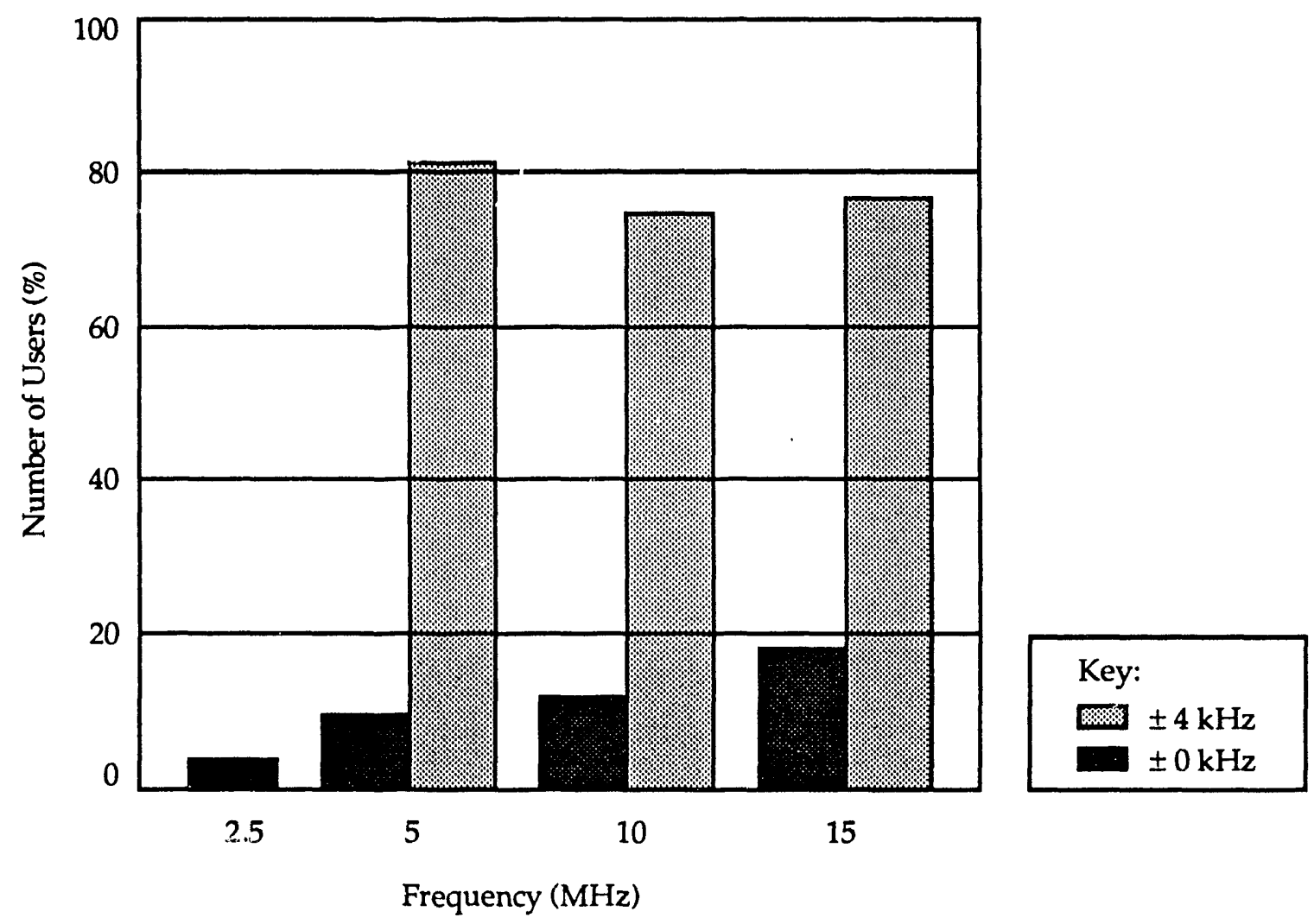

This figure illustrates the much larger usage of the offset MF time and frequency transmissions in the Soviet Union. These frequencies are offset to avoid interference with the rest of the world, which uses the standard CCIR allocations. The problem with this offset approach is that, with the modulation present, it causes the broadcast frequencies to spill over into the guard-band where there are to be no transmissions.

Figure V.1

Use of Frequencies According to the USSR Survey

\section{B. INTRODUCTION}

In a fundamental sense, the transferring of accurate and precise time and/or frequency from some primary standard (point A) to a remote or secondary standard (point B) can be accomplished in two ways: (i) by electromagnetic signal, or 
(ii) by transport of a physical time and/or frequency standard. There are systems where both methods are employed-GLONASS is a classic example. In either case, we have to concern ourselves with measurement noise and measurement inaccuracies. Measurement systems will be discussed in Chapter VI, which discusses time and/or frequency stability, precision, and accuracy of comparison or dissemination systems.

Using electromagnetic signals to transfer time and frequency, one must account for the propagation delay time of the signal from the primary standard to the remote standard or site. The choices for accounting for this delay are to calculate and/or measure it within certain assumptions related to limitations of the system and signal-to-noise considerations. The signal-to-noise considerations are more a part of the measurement system and are addressed in Chapter VI. The accuracy with which this delay is calculated determines the time-transfer accuracy. The unaccounted for changes in the delay time determine the limit for the time stability of the technique. Since time is the integral of frequency, the accuracy of transferring frequency is limited by the unaccounted for time delay instabilities. A typical time and frequency dissemination system involves a transmitter located at point $A$ with a receiver at point $B$. Some techniques are amenable to measuring the path delay by turning the signal around and returning it the same way it came. If the path is reciprocal, the round-trip time can be divided by two in order to estimate the one-way delay.

If transport of a physical time and/or frequency standard (the second method) is used, then the time accuracy is limited by the unaccounted time and/or frequency instabilities in the portable clock or frequency standard over the time of transport. Highly accurate atomic clocks are cumbersome, heavy, and expensive. Over the last decade, the West has been replacing its portable clocks (typically cesium) with satellite time-transfer techriques.

One technique which has proven particularly useful is the GPS commonview technique. Figure V.2 illustrates how this technique works. For a preprogrammed interval, a receiver at site $\mathrm{A}$ records the time difference between its clock and GPS system time, while at the same time, a similar measurement is made at site $B$. The time differences measured at each of the two sites are subtracted. The satellite's clock time (GPS system time) drops out in the subtraction, 
leaving the time difference between clock A and clock B plus the unknown differential delays between the two paths at the time of the measurement. This technique has provided roughly 10 nanoseconds of time accuracy and a few nanoseconds of time stability for international time-difference comparisons.

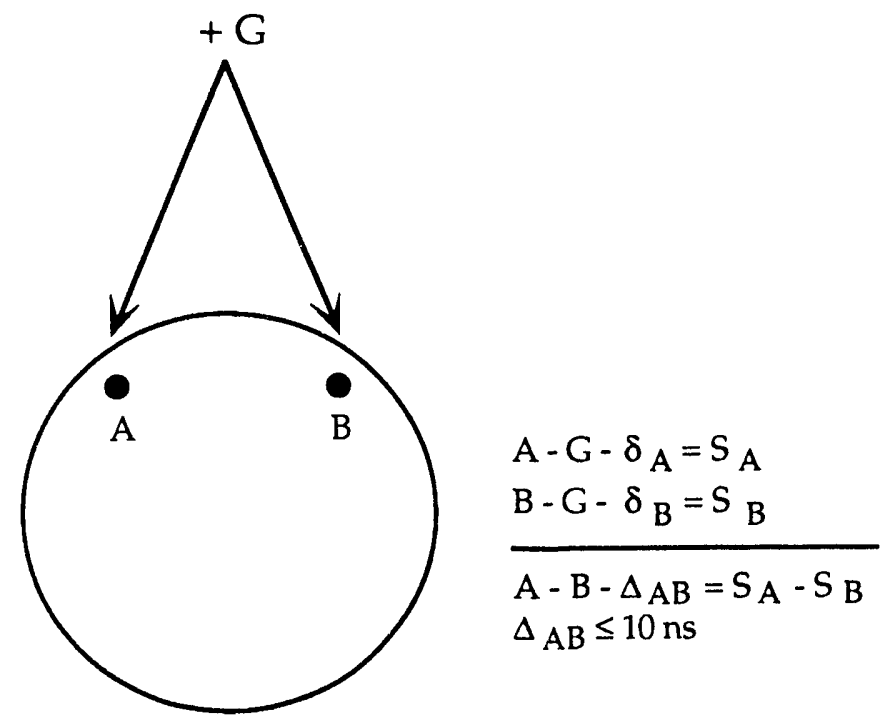

This figure illustrates the leverage gained by using GPS transmissions in common view and available simultaneously at two sites. An international tracking schedule has been developed and is conformed with by the national timing centers having GPS receivers in order to take advantage of this approach. The Soviet Union does not yet participate in this technique because it does not have a GPS receiver at VNIIFTRI (the Physical-Technical and Radio Engineering Measurements All-Union Scientific Research Institute). GLONASS could be used in a similar way, and experiments are now in progress to tie the VNIIFTRI primary cesium beam frequency standards and hydrogen maser clocks into Europe using a combination of GLONASS and GPS. Here, G is the GPS satellite and A and $B$ are the timing centers being compared. The advantage of the common-view technique is that the time difference $S_{A}-S_{B}$ has much less error than the contributing terms, $\mathrm{S}_{\mathrm{A}}$ or $\mathrm{S}_{\mathrm{B}}$. The daily stability of $\triangle_{\mathrm{A}} \mathrm{B}$ from the sidereal daily average optimally weighted across all available GPS satellites approaches a magnitude of 1 nanosecond.

\section{Figure V.2}

\section{GPS Common-View Technique}

As precisions and accuracies have approached the nanosecond level, it has become necessary to include the proper relativistic terms for consistency in the solutions. Soviet scientists seem to be congruous with the West in this area, and often reference US work. 
In the last four decades, time metrology has moved from the millisecond level to the picosecond level. Frequency metrology has progressed from nine significant digits to 16 . The ability to synchronize remote clocks has also improved dramatically. With the availability of GPS, the full long-term frequency stability, as well as the frequency accuracy of the best atomic clocks, can now be transferred to remote sites. The selective availability (SA) of GPS, an intentional degradation of system performance by the US Department of Defense, will adversely affect the accuracy and stability of GPS time and frequency for the average civilian user. As GLONASS becomes available, if the Soviet Union does not degrade its signal, it could take away a significant portion of the international civilian user market; this is discussed further in a later section.

Time transfer systems (or clock synchronization systems) are of ten characterized by a single number, designating a precision or an accuracy of some number of microseconds or nanoseconds. Because this is ambiguous, the following discussion is intended to clarify how time and frequency dissemination systems can be characterized. Some systems require network synchronization in which all of the clocks within a set of nodes agree with respect to each other within some confidence interval. In other systems, the time difference between the nodes can be arbitrary but may need to be stable about a constant offset. Usually network syntonization (same frequency) is needed, as in communication systems. However, there are cases where remote clocks need to be tied to the time and/or frequency of a primary standard, such as with the Universal Coordinated Time (UTC) system. Each country generates its UTC $(k)$ and controls it to be both syntonous and synchronous with the international UTC as generated by the Bureau International de Poid de Mesure (BIPM). The Soviet Union complies with this CCIR agreement.

This chapter is not concerned with measurement noise, that is, divider or counter noise in the laboratory setting, although this can be problematic in some instances. (Chapter VI has some information on this topic.) Instead, it focuses on delay variations and knowledge of absolute delays in time and frequency dissemination systems. As clocks continue to improve, more attention must be paid to the characterization of both the measurement or comparison systems and the dissemination systems. This is especially true if the clocks are remotely 
located from each other. Characterizing the measurement system is essential if a remote (slave) clock is intended to be optimally synchronized or syntonized to a master clock. In the latter situation, optimum design of the servo system locking the slave to the master clock requires a characterization of all of the contributing elements. The published literature indicates that Soviet researchers are only partially addressing these issues.

A free-running clock can almost always be better characterized than one whose output is servo controlled to another clock. Therefore, a computed output or an external micro-phase stepper is useful in providing a synchronized or syntonized output which does not perturb the free-running clock. ${ }^{4}$ A local set of clocks can be better characterized if there are at least three clocks of approximately the same quality. ${ }^{5}$ Once a set of clocks is available, algorithms can be employed to intelligently combine their readings so that the algorithm-computed time and/or frequency can be more stable than that of the best clock in the set. In addition, algorithms can be designed to test for abnormal clock behavior and to desensitize the computed time to any abnormal behavior as well as failures. 6 None of these issues were addressed in any of the Soviet literature reviewed. With the exception of time transfer via meteor trails, the United States appears to be well ahead of the Soviet Union in this area.

Figure V.3 illustrates a straightforward comparison system which measures the time and frequency differences between clock 1 and clock 2 . These can be remotely located as before, at points $\mathrm{A}$ and $\mathrm{B}$, connected by some time and/or frequency dissemination technique. Our concern is the characterization of the overall noise in the comparison including measurement noise, clock noise, and noise introduced in the comparison or dissemination path and system. Figure V.4 illustrates an additional concern which arises in designing a servo

4 D. W. Allan, M. A. Weiss, and T. K. Peppler, "In Search of the Best Clock," IEEE Trans. on Instrumentation and Measurement, IM-38, (1989), 624-630.

5 D. W. Allan, "Time and Frequency (Time-Domain) Characterization, Estimation, and Prediction of Precision Clocks and Oscillators," IEEE Trans. on Ultrasonics, Ferroelectrics, and Frequency Control, UFFC-34, (1987), 647-654.

6 M. A. Weiss, and T. Weissert, "A New Time Scale: AT1 Plus Covariance," Proc. 20th Annual Precise Time and Time Initerual Flärinitiz Mitg., Redondo Beach, California, (30 November1 December 1989). 
loop to slave a remote clock to a master clock. The data from the comparison may not be available immediately; therefore, in the feedback loop the measurement noise, path deviations, and the delay in acquiring the comparison data will fundamentally affect the servo design. Practical delays in acquiring comparison data range from milliseconds to times longer than a month. For example, the delay time (data acquisition time) for servo controlling Coordinated Universal Time at the National Institute of Standards and Technology (UTC NIST) to the international UTC scale is more than a month. Although servo design theory is beyond the scope of this chapter, it should be stressed that the measurement noise, the path noise characteristics, and the delay in acquiring comparison data play important roles in servo design. The above issues are addressed in part with the meteor trail technique, with the system clocks in GLONASS, and with the clocks associated with their communications system.

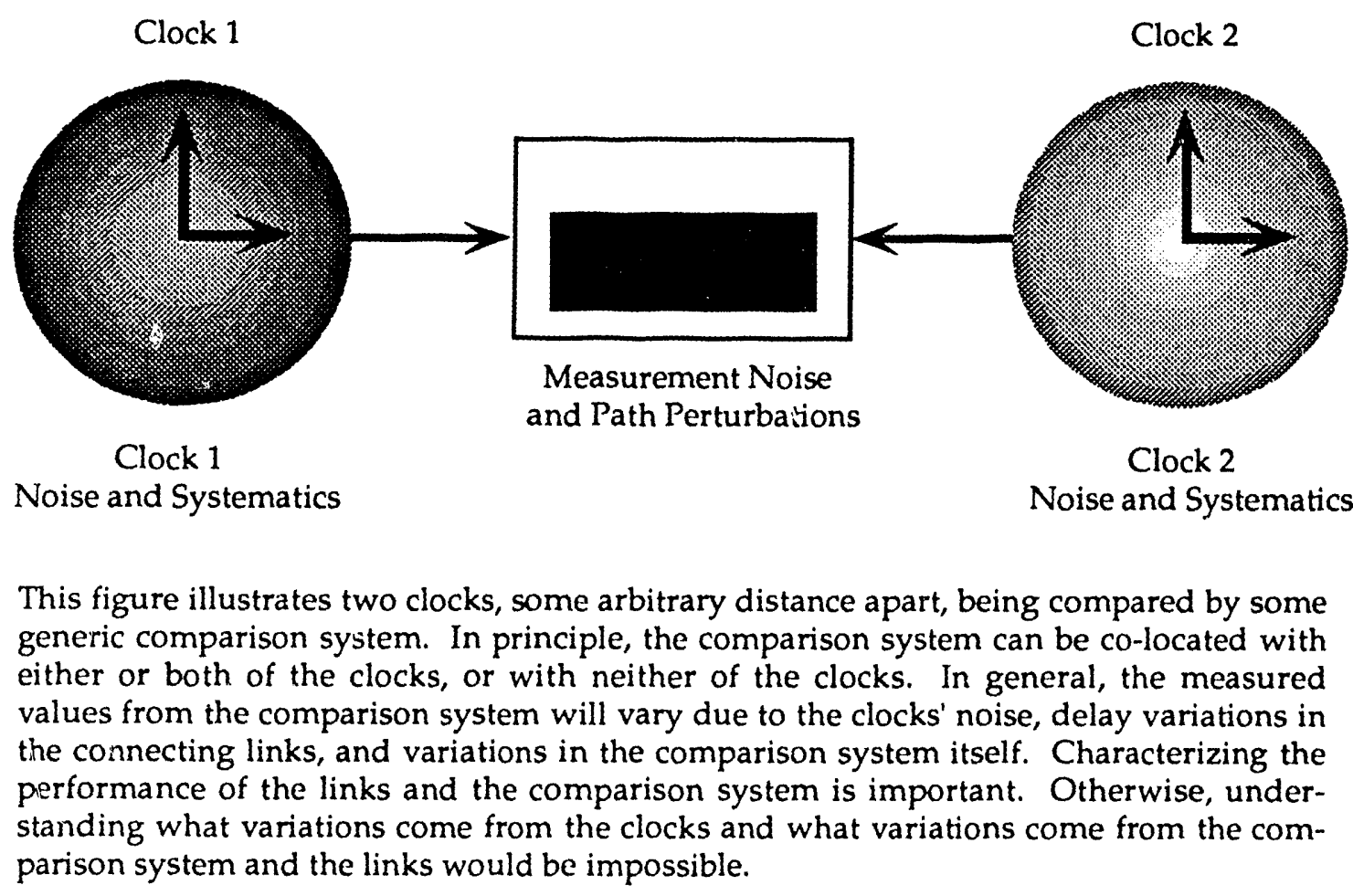

Figure V.3

Straightforward Comparison System 


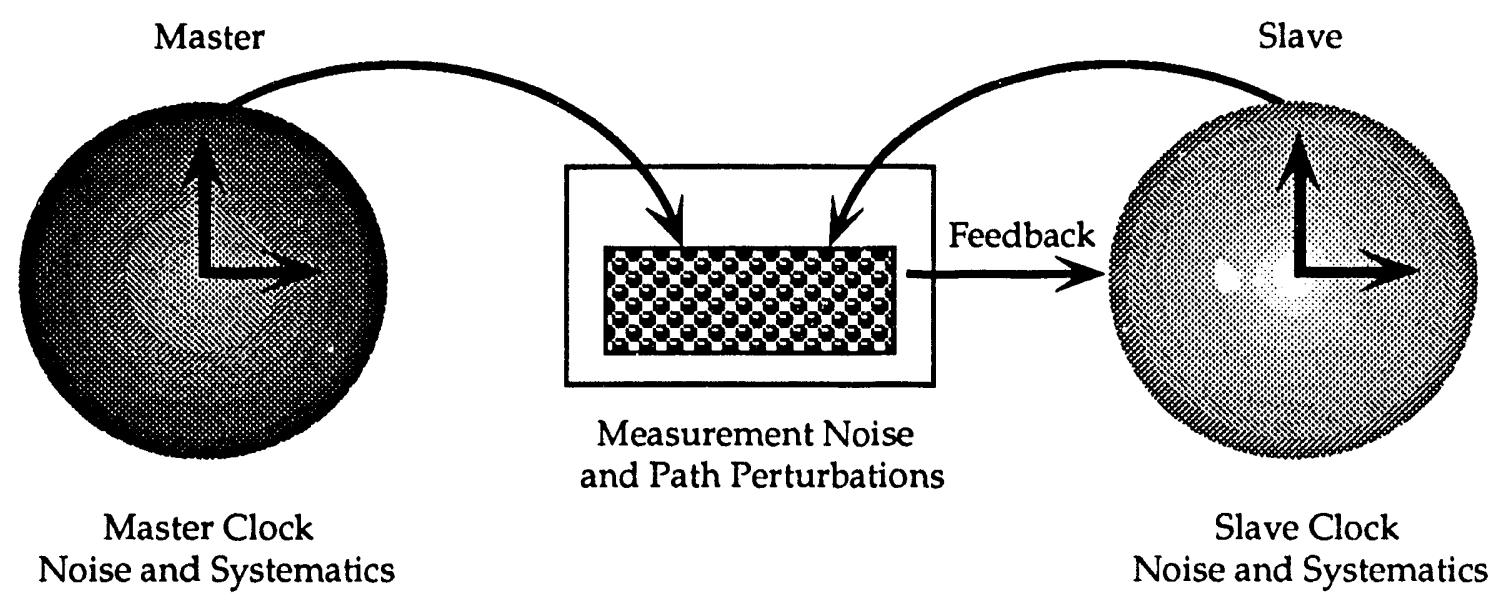

This figure is very similar to Figure V.3. In this case, the goal is to servo control the time and/or frequency of the slave to the master. A proper characterization of the links between the clocks in combination with the comparison system is essential for the proper design of a feedback system to control the slave clock. Another important parameter for the feedback design is the delay associated with the comparison system. This figure also characterizes dissemination systems. The master clock is the transmitter and the slave clock is part of the receiving system.

\section{Figure V.4}

\section{Dissemination or Slave-Locked System}

As previously mentioned, when characterizing systems for comparing clocks that are remotely located to each other, it is important to consider concepts such as time accuracy, time stability, time-prediction error, frequency accuracy, and frequency stability. Each of these concepts has a different and important meaning to the considerations in this chapter.

Conceptually, time accuracy is the tirne difference between the readings of two clocks at some time in a given reference frame. One of the clocks is often defined as perfect (or the master or standard clock) so that the accuracy of a clock is assessed relative to some "ideal" clock or reference time. One can imagine the transport of a perfect portable clock to accomplish this time-difference measurement. Time accuracy is often limited by systematic errors, such as uncertainties in cable delays and propagation path lengths. These can be difficult to measure or assess. 
Time stability is often affected by environmental variations (which affect clock performance as well as dissemination medium). The usual kinds of random variations are often considered in regard to clocks only. However, similar concepts can be carried over to characterize the path-delay variations. Time stability is commonly measured as the root-mean-square (RMS) of the time residuals taken around a linear regression to the time deviations. Given the kind of random variations seen in clocks and dissemination medium, this practice can be very misleading. If there are periodic terms affecting a timecomparison system, then measuring the spectral density of the time or the phase fluctuations is useful. As with clocks, the time deviations of dissemination systems, in general, can be measured using the Allan variance. The effects of these periodic terms can also be measured using $\sigma_{y}(\tau) .5$ For time stability there is often a $\tau$ (averaging-time) dependence, as there is for frequency stability. This $\tau$ dependence for time stability characterization of dissemination or comparison methods is an important consideration that the Soviet Union apparently does not recognize. During panelist David Allan's September 1989 visit to VNIIFTRI (the Physical-Technical and Radio Engineering Measurements All-Union Scientific Research Institute), Soviet researchers stated that they were not aware of this technique, and nothing in the Soviet literature suggests such knowledge. This technique has only limited recognition in the West.

The quantity $\mathrm{K} \tau \sigma_{\mathrm{y}}(\tau)$ is a useful measure for estimating the optimum timeprediction error in a comparison. Often, a particular power-law spectral density process is the dominant model for the signal variations from the clocks and/or the comparison or dissemination system. Optimurn means to minimize the squared error of prediction given a certain power-law spectral density model. The value of $K$ is $1 / \sqrt{3}$ for white-noise phase modulation (PM), 1 for white-noise frequency modulation (FM) and for random-walk FM, and 1.2 for flicker-noise FM under the assumption of optimum prediction. Sometimes, white-noise phase modulation is the predominant noise model for either clocks or dissemination systems, in which case the quantity $\sigma_{x}(\tau)=\tau \bmod \sigma_{y}(\tau) / \sqrt{3}$ approximates the optimum RMS time-prediction error for an average over $\tau$ of $x(t)$ measurements. ${ }^{5}$ While this white-noise PM model is usually applicable only to clocks for short sampling times on the order of seconds out to minutes, it is sometimes 
applicable for sampling times of days and even weeks for some dissemination and comparison systems.

Frequency accuracy for a given primary standard is not a function of integration time and is properly stated as a single number. But the ability of a comparison system to determine absolute frequency difference between two standards is often a function of the sampling average or integration time, $\tau$. The frequency accuracy of a comparison or dissemination system is also a function of the data processing method. This leads to the idea that there is an optimum method for estimating the absolute frequency difference between two remote clocks or for controlling the frequency of a remote clock. This idea is being developed in the United States, and appears to be only partially appreciated in the Soviet Union (Koshelyayevskiy and Pushkin, 1990).

Frequency stability, similar to time stability, is observed by looking at a plot of the fractional frequency offset, $y(t)$, where $y(t)=\left(v(t)-v_{0}\right) / v_{0}$, with $v(t)$ being the time-varying frequency output of a clock and $v_{0}$ being the clock's nominal frequency. In practice, measured values of $y(t)$ are observed over some averaging time, $\tau$. It is often useful to observe a $y(t)$ plot at different averaging times. The frequency stability of a comparison system can be quantified in the same way clocks are characterized, using an $\sigma_{y}(\tau), \sigma_{x}(\tau)$ or $\bmod \sigma_{y}(\tau)$ plot. The time stability of a dissemination or comparison system is best characterized using an $\sigma_{\mathbf{X}}(\tau)$ plot. It is sometimes useful to measure the spectral density of the frequency fluctuations to supplement the above time-domain methods in order to quantify periodic variations. The kind of noise observed in comparisons between two clocks and which may be added by the comparison system will determine how to optimize estimates of characterization parameters (both systematic and noise) for the clocks and the comparison system. One important example of a characterization parameter is the frequency drift between two clocks. Although the Soviet literature does not address the above issues that are important in network synchronization, it is highly probable that the Soviet Union is doing this type of work. There are important relationships between time accuracy, time stability, time prediction error, frequency accuracy, and frequency stability. Soviet researchers seem to appreciate some of these issues, but not all. 
In the past, the accuracy of operational comparisons between remote clocks and dissemination systems fell behind the accuracy of the standards themselves. During the 1980s, the development and application of two-way satellite and GPS time transfer dramatically changed the picture. With the excellent comparison accuracy available with the GPS common-view technique, comparison accuracy is now leading clock accuracy. This was a major breakthrough for international time and frequency comparisons. The GPS common-view technique is now the de facto international standard for comparisons. However, the Soviet Union has been left out of these comparisons-apparently having no GPS receiver. At the April 1989 Consultative Committee for the Definition of the Second (CCDS) it was agreed that the Soviet Union would set up GLONASS receivers at VNIIFTRI in order to perform common-view measurements with the University of Leeds. Leeds, in turn, has developed GLONASS receivers and has GPS receivers, and has agreed to be the point of comparison, as they also perform common-view measurements with BIPM near Paris and with the National Physics Laboratory (NPL) near London. The long-term stability of the primary cesium beam frequency standards at VNIIFTRI appears to be excellent-a few parts in $10^{14}$ over the course of a year (Belotserkovskiy and Paliy, 1972; Koshelyayevskiy and Pushkin, 1990). If this measurement system, as outlined above, is established in the Soviet Union, their primary standards could make a valuabie contribution to International Atomic Time (TAI) and UTC.

N. B. Koshelyayevskiy, a senior scientist in time and frequency at VNIIFTRI, visited NIST in Boulder, Colorado, in April 1991 and reported on the progress of this work. Data on the VNIIFTRI clocks are now being shared with the West on a regular basis. The plan, as suggested at the last CCDS using GLONASS, is being followed with one important exception. The GLONASS tracking schedule for VNIIFTRI is configured for synchronizing clocks across the territory of the Soviet Union. The schedule at Leeds, England, is configured to the East of them for best comparison with VNIIFTRI. Since these schedules are different, some measurement noise is introduced. If a weighted average on an exact common-view set of data were followed, this measurement noise could be reduced considerably. This would require closer cooperation of the staff at VNIIFTRI with the West. W. Lewandowskiy of the Time Service Section at BIPM plans to visit VNIIFTRI to coordinate this effort. 
A decision by GPS system operators to intentionally degrade performance as observed by civilian users-the so-called process of selective availability-raises questions which are important to time-transfer applications. This can seriously impact the international time-comparison system using GPS in common view. If GLONASS remains undegraded, both the civilian timing community as well as a large number of other GPS users could end up using GLONASS receivers. It is apparent that the Soviets are eager to have GLONASS receivers manufactured abroad.

In the United States, there is a Civil GPS Service (CGSC) committee, which provides guidelines for, among other things, dealing with selective availability (SA). These guidelines may be sufficiently effective that the sale of GLONASS receivers in the West may not be an economic threat. There are two parts to SA: (i) clock dither (an onboard intentional modulation of the GPS space vehicle's clock output signal), and (ii) "epsilon" (an intentional degradation of the broadcast satellite ephemeris [satellite position]). When SA was officially turned on (25 March 1990), it was apparent from the international time comparisons that little or no "epsilon" was present in the space vehicle's signals. The implications of this are very significant because, in the common-view time-transfer mode, the clock dither totally cancels. Therefore, during 1990, SA had no deleterious effect on international time comparisons. At the outset of the Persian Gulf crisis, SA was turned off. The plan of the Department of Defense (DoD) is to have the option to turn SA back on and also to include "epsilon" in the SA civil usage degredation at some future date appropriate to DoD needs and strategy. The CGSC, in coordination with the DoD, is working to provide a post-precise ephemeris so that both dither and "epsilon" can be dealt with if SA is turned back on. Under these conditions, the civil community will still have the full accuracy of time comparisons using GPS in common view.

Time transfer using the two-way satellite technique now appears to be an increasingly attractive technique available to primary timing centers. More information is needed on the accuracy and long-term time stability of this comparison technique, as early work has not focussed on these. ${ }^{7}$ Most of the pub-

7 D. Hanson, "Fundamentals of Two-Way Time Transfers by Satellite," Proc. 43rd Annual Symp. on Frequency Control, (31 May-2 June 1989), 174-178. 
lished results are on short-term time stability. This mode has now been operational for about two years between the US primary timing centers UTC(NIST) in Boulder, Colorado, and UTC(USNO) in Washington, DC (a distance of roughly 3 megameters, or 1,700 miles). This technique has better second-to-second time stability than GPS, and the longer-term stability also should be better, but this is still being assessed.

Important factors for all of these comparison and dissemination systems include cost, ease of use, and means for assessing comparison accuracy. An ideal comparison system would have negligible measurement noise, and an ideal dissemination system would have negligible deterioration of the time and/or frequency signal during the time of propagation and would provide the time for dissemination so that it could be compensated. There is currently no single system that meets either of these ideals. In practice, having the comparison or dissemination system properly characterized in terms of delays and delay deviations is essential in order to know the uncertainties associated with the corresponding time and frequency measurements. For completeness, the time and frequency accuracies, as well as the time and frequency stabilities, need to be known.

Figure V.5 is a plot of some of the more commonly used comparison techniques. Both $\sigma_{\mathrm{y}}(\tau)$ and $\bmod \sigma_{\mathrm{y}}(\tau)$, the IEEE accepted standards, are used to characterize the frequency stability of these comparison systems because, in some cases, white-noise phase modulation (PM) is the limiting random process and $\sigma_{y}(\tau)$ characterization is ambiguous for that process. In Figure V.5, the stabilities of various techniques are characterized using $\sigma_{y}(\tau)$ except where indicated by an asterisk. Mod $\sigma_{\mathrm{y}}(\tau)$ was used in those cases where white-noise PM was predominant for some range of sample times, $\tau$; an asterisk marks those cases.

D. A. Howe, D. Hanson, J. Jespersen, M. Lambardi, W. Klepczynski, P. Wheeler, M. Miranian, W. Powell, J. Jeffries, and A. Meyers, "NIST-USNO Time Comparisons Using Two-Way Satellite Time Transfers," Proc. 43rd Annual Symp. on Frequency Control, (31 May-2 June 1989), 193-198.

M. Imae, H. Okazawa, T. Sato, M. Urazuka, K. Yoshimura, and Y. Yasuda, "Time Comparison Experiments with Small K-Band Antennas and SSRA Equipments Via a Domestic Geostationary Satellite," IEEE Trans. on Instrumentation and Measurement, IM-32, (1983), 199-203. 


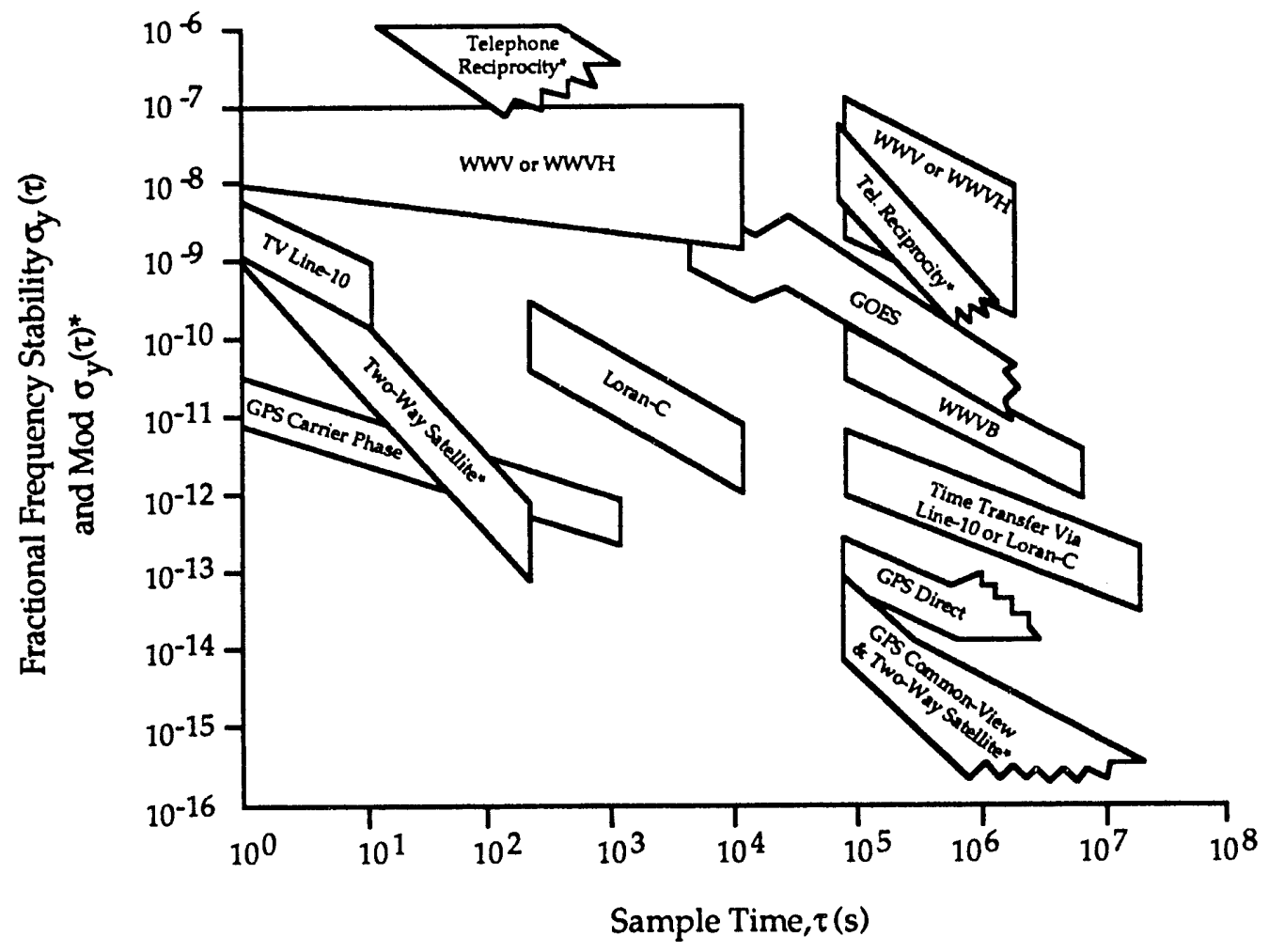

Figure V.5

Nominal Fractional Frequency Stability of Possible Frequency Calibration Methods

The telephone reciprocity data were analyzed under the assumption of reciprocity of the path (to calibrate the path delay, measure the round trip time and divide by two). 8 The short-term data were measured locally; the long-term data were measured between Colorado and Hawaii via a communication satellite. The use of telephone reciprocity appears to have a bright future in the West because of its simplicity and cost effectiveness; there is no evidence that the Soviet Union has done anything with this technique.

8 D. W. Allan, D. D. Davis, J. Levine, M. A. Weiss, N. Hironaka, and D. Okayama, "New Inexpensive Frequency Calibration Service from NIST," Proc. 44th Annual Symp. on Frequency Control, Baltimore, Maryland, (23-26 May 1990), 107-116. 
The WWV and WWVH time and frequency transmissions at 2.5, 5, 10, and $15 \mathrm{MHz}$ (WWV also broadcasts at $20 \mathrm{MHz}$ ) are limited in their stabilities by skywave path variations. GOES East and GOES West are NOAA weather satellites broadcasting UTC(NIST) on two slightly different frequencies near $468 \mathrm{MHz}$. Here, the stability is limited by the knowledge of the satellites' ephemerides. WWVB is NIST's $60-\mathrm{kHz}$ time and frequency broadcast service; in this case, the propagation path stability is limited by the fluctuations in the Earth-ionosphere waveguide.

The TV Line-10 method involves line-of-sight transmissions in the TV band. It can operate with an atomic clock at the transmitter, or with two clock sites receiving the TV Line-10 arrival times concurrently and subtracting one set of numbers from the other. Stability limitations often are caused by the receiving equipment.

Loran-C is a ground-wave navigation signal (at $100 \mathrm{kHz}$ ) operated by the US Coast Guard. The time is monitored and controlled with respect to UTC(USNO). The stability is primarily limited by propagation path variations.

Two-way satellite time transfer uses spread-spectrum modems operating with different up-link and down-link carrier frequencies in one of several different bands $\left(C, K_{u}\right.$, and $\left.K\right)$. The short-term stability for two-way satellite time transfer is limited mainly by signal-to-noise and bandwidth considerations. Currently, the long-term performance seems to be limited by equipment instabilities. One can only extract frequency information from the "GPS Carrier Phase" measurements, and the stability seems to be limited by the GPS onboard clocks. Time and frequency stability of directly received GPS signals is limited mainly by variations in the GPS Kalman state estimates for the system. If one is using an L1 GPS timing receiver only, then the ionospheric modeling errors can contribute additional instabilities. In some cases, signal multipath errors and/or receiver instabilities can also contribute significant instabilities. Using GPS in the common-view mode cancels out the clock instabilities of the GPS satellites and some of the broadcast satellite-ephemeris instabilities. The stability limits for the common-view mode arise from the same mechanisms as for GPS direct measurements except that some of the mechanisms are reduced by commonmode cancellation. 
Because of the Persian Gulf crisis, a more detailed discussion of some of the different ways GPS or GLONASS can be used for precise and/or accurate time and frequency is provided below. There are some who believe that adversarial third-world countries could use accurate timing for hostile activity against the United States, for example, for missile tracking. Because the Soviet Union claims that it will not have SA on GLONASS, for the sake of relevant discussion, it will be assumed that $\mathrm{SA}$ is turned off in the following discussion.

Besides the common-view technique, there are three additional ways of using GPS (and GLONASS) to provide accurate and precise time and frequency. The first technique is direct access to the signal. Knowing a receiver's position will yield GPS system time with an accuracy to UTC of about 1 microsecond. If two additional broadcast data words are used ( $\mathrm{A} 1$ and $\mathrm{A} 2$ ), then time can be known to better than 30 nanoseconds, and the day-to-day time stability is about 10 nanoseconds. The normalized frequency stability, $\sigma_{y}(\tau)$, of this approach is less than $1 \times 10^{-13}$ with $\tau$ in the range of a day to a few weeks. The frequency accuracy is at about the same level. The accuracy of this same approach with GLONASS is now jointly being studied by the University of Leeds and the National Institute of Standards and Technology.

The second technique is the clock fly-over approach. Here, the GPS or GLONASS satellite clock can be thought of as a portable clock. It goes over station A, then some time later orbits in view of station B. Stations A and B compare their readings to estimate the time difference, $A-B$. This method has an advantage over the common-view approach in that sites $\mathrm{A}$ and $\mathrm{B}$ do not have to be in simultaneous common view of a given satellite. For the number of satellites now available, A and B can be anywhere on the Earth. The errors and uncertainties for this approach will be the same as for the common-view technique, except that the deviation of the time and/or frequency of the satellite clock between observations at sites A and B will contribute to the uncertainties, as will the deviation in the different broadcast ephemeris at the two different times. The lack of predictability of the satellite clock over 12 hours is typically less than 10 nanoseconds, and the deviation in the broadcast ephemeris uncertainties is about the same amount. The Soviet Union has already used this technique in 
comparisons with ground portable hydrogen maser clocks and has obtained agreement to about 50 nanoseconds.

The third technique, developed for geodesy, is the most accurate, but it only works over relatively short baselines. In addition to its high accuracy, it can also operate independently of SA. It is patterned after the very long baseline interferometry (VLBI) technique, which uses radio stars to determine baseline movements over long baselines (intercontinental). In the case of GPS, four satellites are viewed simultaneously from two sites, $A$ and $B$. The received signals at $A$ and $B$, which are about $10^{5}$ times brighter than for VLBI radio stars, are later cross-correlated to determine their relative positions and time differences. Uncertainties of a few centimeters on position and about 0.1 nanosecond are attainable over thousands of kilometers. Time accuracies of about 1 nanosecond are possible. This type of comparison cannot be accomplished in real time.

Linear regression analysis of ten is used to analyze processes which do not have a white spectrum for the residuals. In this case, the linear regression coefficients and their confidences often can be very misleading. A mod $\sigma_{y}(\tau)$ diagram will indicate whether or not it is legitimate to use linear regression analysis, and, if not, then it gives a measure of the effects of the degradation caused by the actual deviations on the estimate of the frequency difference between the two remote clocks.

Figure V.6 is a plot of the RMS time-prediction error seen in currently available clocks and oscillators. The data have been used in an optimum way to predict the future over an interval, $\tau_{\mathrm{p}}$.

Figure V.7 is a plot of $\sigma_{x}(\tau)$ as a function of $\tau$. With $\tau=n \tau_{0}$, this shows whether or not there is any benefit from averaging $n$ values of the $x(i)$ time-difference measurements. One advantage of this approach is that it illustrates the benefit of averaging the time-difference measurements, whether or not the instabilities are in the comparison system or in the clocks. If the measurement noise residuals are a white PM process, then the time stability will improve as the square root of $\tau$. If it is a flicker PM process, there will be no improvement with averaging. If the plot degrades with increasing $\tau$ (slope greater than 0 ), then there are probably non-stationary processes perturbing the comparison system. 
In the case of Loran-C, a double hump can be seen at one-half day and at one-half year caused by diurnal and annual variations. Loran- $C$ has been the primary link between the Soviet Union and the rest of the world, and the annual variations have been the principal limitation in the ability of the rest of the world to observe and utilize the performance of Soviet primary frequency standards.

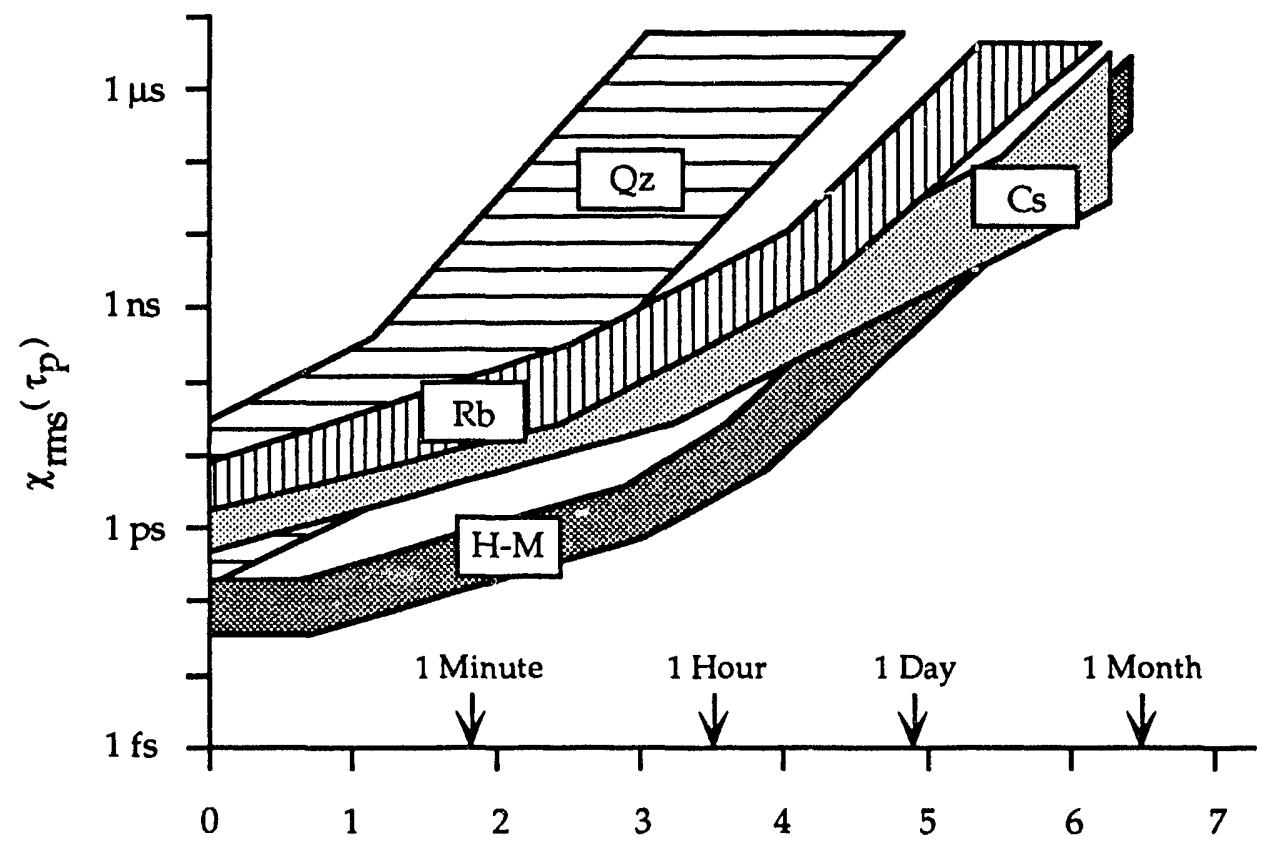

Log Prediction Interval, $\tau \mathrm{p}^{(\mathrm{s})}$

This plot illustrates the time-prediction error, $\chi_{\mathrm{rms}}\left(\tau_{\mathrm{p}}\right)$, as a function of the prediction interval for commercially a vailable precision clocks. Here, $\mathrm{Qz}=$ quartz crystal oscillator clock; $\mathrm{Rb}=$ rubidium gas cell frequency standard clock; $\mathrm{Cs}=$ cesium beam frequency standard clock; and $\mathrm{H}-\mathrm{M}=$ active hydrogen maser clock. This prediction error is calculated from $\mathrm{K} \tau \sigma_{\mathrm{y}}(\tau)$ with $\mathrm{K}$ being chosen for an optimum prediction estimate. The value of $K$ depends on the type of noise.

\section{Figure V.6}

\section{RMS Time-Deviation Error}

Scientists in the West have longer-term common-view data using GPS than is available from GLONASS. As seen in Figure V.7, the time stability does not continue to improve as the square root of $\tau$. In this case, the nonstationary processes causing these degradations are most likely related to ionospheric modeling errors and errors in the estimates of the satellites' ephemerides. The time insta- 
bilities due to GLONASS space-vehicle clock noise and uncertainties in GLONASS ephemerides seem to be significantly larger than those in GPS, as seen in the data from the University of Leeds. 9 Therefore, the common-view timetransfer instabilities and inaccuracies are larger for GLONASS than those measured for GPS. 10

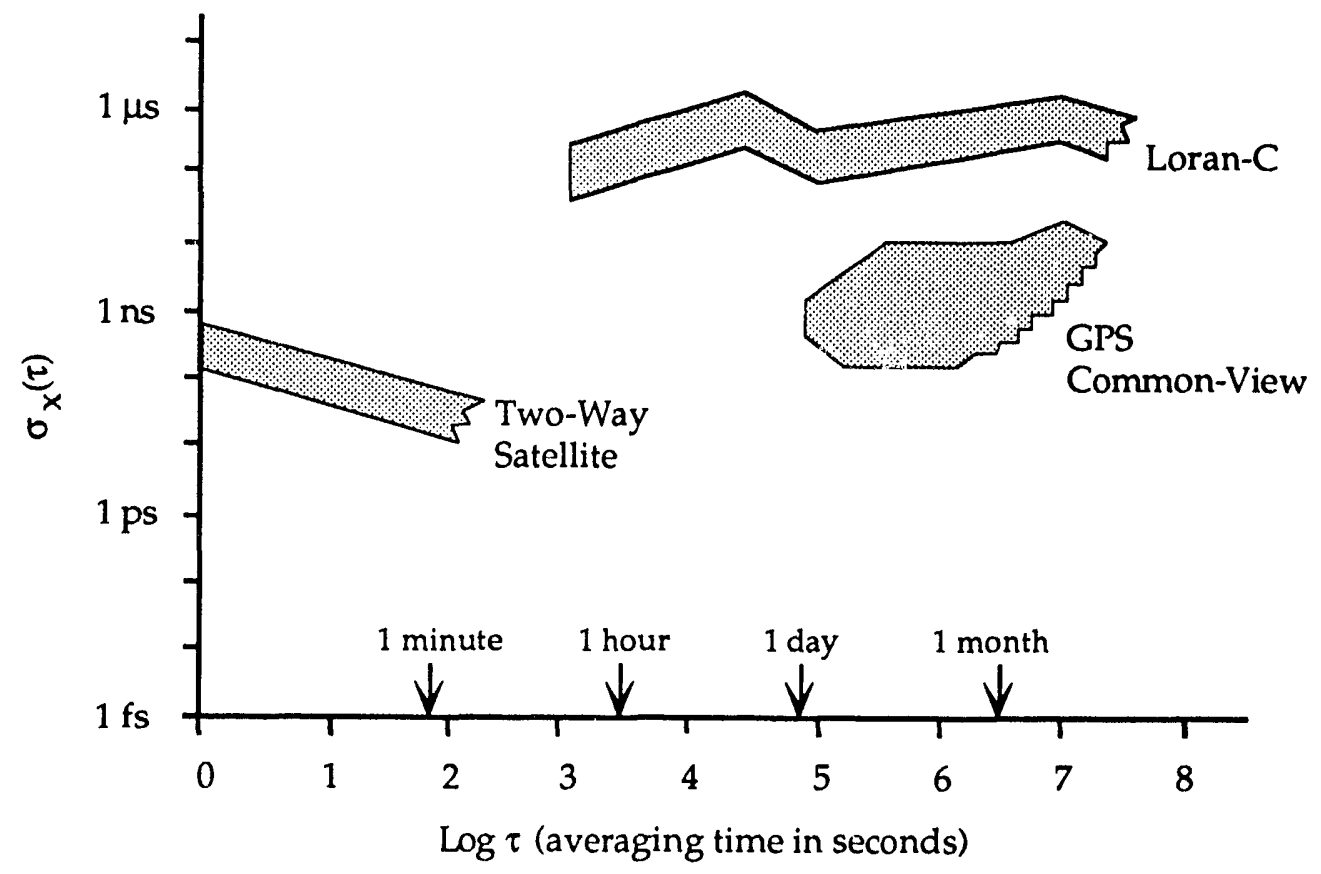

This type of plot can be used to determine whether or not smoothing or averaging the data is beneficial. The time stability is defined as the product $\tau \bmod \sigma_{y}(\tau)$. For flicker noise PM, white-noise FM, flicker noise FM, and random-walk noise FM, the standard deviation of the time residuals grow without bound as the data length increases. Therefore, it is not a good measure. The above product is a good measure, if convergent, and is data-length independent. This measure can also show the systematic effects of environmental perturbations, as well as the different kinds of noise processes that may be driving the instabilities in the comparison system and/or in the clocks.

\section{Figure V.7}

\section{RMS Time Estimate Due to Random Noise}

9

P. Daly, paper presented at the 1989 Institute of Navigation Conference in Colorado Springs, Colorado.

P. Daly, D. W. Allan, I. D. Kitching, and T. K. Peppler, "Frequency and Time Stability of GPS and GLONASS Clocks," Proc. 44th Annual Symp. on Frequency Control, Baltimore, Maryland, (23-26 May 1990), 127-139. 
Multipath distortion effects at the antenna can sometimes cause several nanoseconds of bias in the time inaccuracy in the GPS common-view approach. However, these distortions do not change the slope in this type of plot if the bias is constant. Therefore, the accuracy of frequency transfer is not affected. However, the multipath tends to change slowly with time as the satellite constellation moves gradually. This causes very low frequency variations, which are not significantly detrimental at current levels of performance of atomic clocks. In the future, multipath will become more of a problem; work on this problem is being conducted in the United States. This is a significant problem for GLONASS because of the lower elevation of the Soviet satellites where the multipath effects will be different from day to day and can be expected to repeat every eight days.

For two-way satellite time transfer, the noise limit does not continue to decrease, as indicated by the short-term results in Figure V.7. Daily deviations on the order of a few nanoseconds have been observed, but these will likely be reduced as the systems are improved and better characterized. This characterization of the two-way satellite time-transfer technique will be important in the future, especially for averaging one day and longer. A determination of the time accuracy of this technique will be important as well. Theoretically, both the time stability and the time accuracy of two-way time transfer should provide an excellent means for comparing widely separated clocks. The primary disadvantage of this technique is the need for broadcasting from each station, a requirement which increases cost and involves licensing with government agencies. The Soviet Union has expressed a strong interest in cooperating in some of these comparisons. Future programs undoubtedly will include such experiments. Experiments have already been conducted between the IEN (Instituto Elettrotechnico Nazionale) in Torino, Italy, and two different sites in the People's Republic of China. ${ }^{11}$

It is clear that the best means for comparing widely separated clocks involve satellite techniques. For clocks in close proximity (that is, within a distance of

11 F. Cordara, P. G. Galliano, V. Pettiti, S. Leschiutta, Jin-An Song, Heng-Qio Zheng, DingChang Lou, and Ven-Zhong Liu, "Synchronization Experiment Between the IEN (Italy) and the Shaanxi Observatory (China) Time Scales Via SIRIO 1 Satellite," Proc. 25th Symp. on Space Stations and Their Utilizations (sic), Rome, Italy, (26-28 March 1985), 85. 
about $100 \mathrm{~km}$ ) perhaps optical fibers will provide the best comparisons. In general, the Soviet Union appears to be lagging the United States in both of these developments. The introduction of SA on GPS could adversely change the status of the US lead in the future as it relates to the civil sector.

As more accurate and stable clocks are developed, higher frequencies will need to be used to achieve better phase resolution for the comparisons.

The GPS system could be improved to provide a time accuracy approaching a few nanoseconds (aside from SA). Short baseline comparison studies suggest that accuracies as low as 0.1 nanosecond could be achieved. 12 Time stabilities for GPS common-view comparisons yield $\sigma_{x}(\tau)$ of about 1 nanosecond times $\tau^{-1 / 2}$, where $\tau$ is in days. At $\tau=1$ day, this product actually ranges from 0.8 to 8 nanoseconds for the many international time-stability measurements that use the GPS common-view method. With ionospheric measurements and moreexact post-ephemeris data for the satellites, the GPS common-view technique could yield a comparison limit for frequency accuracy approaching 10-17. This would require approximately three months of integration under the assumption of ideal white-noise phase modulation. Codeless ionospheric measurement receivers, which measure the ionospheric delay along the path, are now becoming available for GPS. It is also likely that precise post-measurement ephemerides will be made available to the civil sector (the non-precise positioning service user). With these advances, the GPS common-view method for time and frequency transfer will improve. ${ }^{13}$

How well the systematics of the two-way satellite timing technique can be understood is yet to be determined. From a theoretical point of view, this technique should be better, in both time stability and time accuracy, than the GPS common-view technique. This method could provide about an order of magni-

12 P. F. MacDoran, D. J. Spitzmesser, and L. A. Beunnagel, "SERIES: Satellite Emission Range Inferred Earth Surveying," Proc. 3rd Int'l. Geodetic Symp. on Satellite Positioning, Las Cruces, New Mexico, (February 1982).

13 W. Lewandowskiy, G. Petit, C. Thomas, and M. A. Weiss, "GPS Time Closure Around the World Using Precise Ephemerides, Ionospheric Measurements, and Accurate Antenna Coordinates," Proc. 5th European Frequency and Time Forum, Besancon, France, (12-14 March 1991). 
tude of improvement. The Soviet Union will probably pick up on this technique-following the lead of the United States and other nations.

An often overlooked experiment that potentially could lead to time-transfer improvement is the Scout Rocket Experiment which involved flight of a hydrogen maser. ${ }^{14}$ This experiment used a microwave Doppler cancellation method and an ionospheric calibration system. According to the published literature, it is estimated that time stability $\sigma_{\mathbf{x}}(\tau)$ over several hours was roughly 10 picoseconds. With this level of stability available from a satellite-born hydrogen maser, cycle ambiguity of the clock's microwave signal could be resolved from pass to pass or from day to day and could yield frequency comparisons over 24 hours of $10^{-16}$. If the residuals for the comparison process were white PM from day to day, it would take only a few weeks to measure a frequency difference at the 10-18 level. At this level, relativity considerations become very important and they will be difficult to calculate. However, with bigger and better computers coming in the future, perhaps the relativity issues will be solvable. This technique was reported at a conference in 1981 where there was a Soviet delegation, but there is no evidence that the Soviet Union is working on this technique. The United States has built and tested the components and has studied the overall technique from a theoretical point of view.

\section{DISSEMINATION SYSTEMS IN THE SOVIET UNION}

\section{Satellite Techniques}

The Soviet literature reveals that there are three satellite dissemination techniques: (i) GLONASS, (ii) television, and (iii) communication systems. Each of these techniques is discussed below.

14 D. W. Allan, C. O. Alley, N. Ashby, R. Decher, R. F. C. Vessot, and G. M. R. Winkler, "UltraAccurate International Time and Frequency Comparison Via an Orbiting Hydrogen-Maser Clock," Journal de Physique, Col!nque C8, (1981), 395-413.

R. F. C. Vessot, M. W. Levine, E. M. Mattison, E. L. Blomberg, T. E. Hoffmann, G. U. Nystrom, B. F. Farrell, R. Decher, P. B. Eby, C. R. Baugher, J. W. Watts, D. L. Teuber, and F. D. Wills, "Tests of Relativistic Gravitation with a Spaceborne Hydrogen Maser," Phys. Rev. Lett., 45, 12(1980), 2081-2084.

R. F. C. Vessot, and M. W. Levine, "A Test of the Equivalence Principle Using a Spaceborne Clock," Gen. Relativ. Gravit., 10 (1979), 181-284. 


\section{a. GLONASS}

The accuracy and stability with which time and frequency can be disseminated via GLONASS satellites is a function of several factors that include the frequency stability and reliability of the clocks on board the satellites, the accuracy and stability with which they can determine the satellites' ephemerides, the accurate measurement and estimation of the propagation delays, the amount of multipath at the antenna, and the receivers' accuracies and stabilities. Once the measurements and models are determined for each of the above contributions, the manner in which these data are processed in a statistical and systeinatic sense will determine the final accuracy and stability of time and frequency transfer and/or dissemination from GLONASS.

In an overall performance sense, at the April 1989 CCDS meeting, Dr. Nikolay Koshelyayevskiy reported a 50-nanosecond accuracy for a GLONASS comparisor against a portable hydrogen maser over a distance of about 3 megameters $(\sim 1,700$ miles). At that time, Soviet scientists apparently had conducted only one experiment. There are currently two GLONASS receivers at the new time and frequency complex at VNIIFTRI.

Table V.1 shows the rapid performance improvement the Soviet Union is achieving with successive GLONASS satellite launches. The numbering in the table is that of Professor Peter Daly at the University of Leeds. The Soviets launch three satellites at a time. The long-term stability and the frequency drift on the later units indicate that they probably use cesium beam frequency standards. This was confirmed at the March 1990 European Frequency and Time Forum.

We have learned that Pïofessor Daly has obtained roughly a 10-meter accuracy on position determination using GLONASS, which gives a good indication of the accuracy limits of the GLONASS ephemerides. This number also gives a nominal value for the time accuracy limit due to this error contribution. Since the satellites are never orthogonal for a navigation or position solution, the time accuracy errors tend to be smaller than one would deduce directly from the 10-meter number, that is, roughly 20 nanoseconds. The time-stability numbers 
obtained by Professor Daly are more like 50 nanoseconds from day to day. In some of their theoretical work, Soviet scientists discuss the potential of being able to determine the ephemeris of satellites to 1 meter (Kholshevnikov et al., 1984).

\begin{tabular}{|c|c|c|c|}
\hline \multicolumn{4}{|c|}{ Table V.1 } \\
\hline \multicolumn{4}{|c|}{ FREQUENCY DRIFT IN GLONASS SATELLITE CLOCKS } \\
\hline Number & Year of Launch & $1 /$ Floor $\left(\times 10^{-13}\right)$ & Drift $\left(1 \times 10^{-15} /\right.$ Day $)$ \\
\hline 16 & 1985 & 30 & +77 \\
23 & 1986 & 10 & -20 \\
37 & 1988 & 2 & -5 \\
\hline
\end{tabular}

Experiments are planned between VNIIFTRI, Leeds, and the BIPM. Professor Daly and David Allan of NIST have combined their efforts to study the onboard clocks and the system time for GLONASS as compared to GPS.10

Plots of GLONASS system time versus UTC(USNO) are shown in Figure V.8. However, GLONASS system time is many tens of microseconds away from UTC, and therefore, more than an order of magnitude less accurate than GPS system time. In addition, it is at the wrong rate and therefore the time inaccuracy is increasing. GLONASS transmits a correction word which brings system time to agree with Moscow time. In turn, Moscow time is supposed to agree with UTC(SU), which is the state standard time from VNIIFTRI. The Soviet intent is to make this correction word bring GLONASS time into agreement with UTC(SU), and they have accomplished this some of the time. UTC(SU) is about 10 microseconds off from UTC and the difference is decreasing. VNIIFTRI currently has no algorithm in place to coordinate UTC(SU), that is, to make it nominally synchronous with UTC. In contrast, UTC(USNO) attempts to stay synchronous with UTC to within roughly a microsecond, and UTC(NIST) has maintained synchronism with UTC to within about $1 / 3$ of a microsecond over the last few years. 


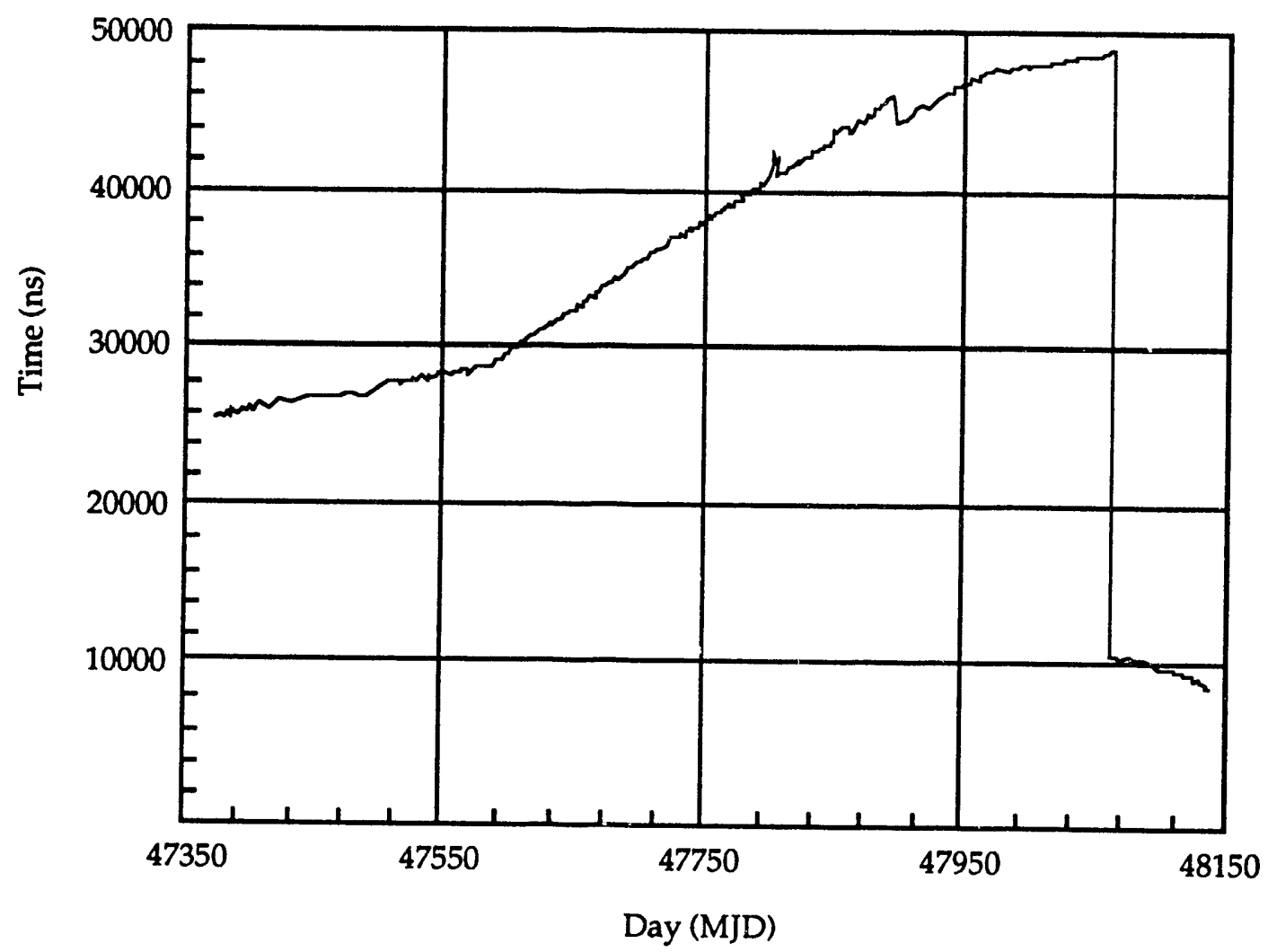

Figure V.8

UTC(USNO) vs. GLONASS System Time

(MJDs 47374 - 48138)

Some data on the stability of GLONASS system time are available. Based on the characteristics of the data, it appears that the Soviet Union has an ensemble of hydrogen masers at the control facility, which is located near Moscow. Soviet researchers apparently use one of the clocks and change the one they use from time to time. During a four-month period (May through August 1989), the performance was spectacular against UTC(USNO). The frequency stability was about 10-14 for integration times on the order of a month, and no apparent frequency drift was observed.10 The data looked as if they were phase locked to UTC(USNO) with a fixed frequency offset, implying that the Soviets have a GPS receiver; Soviet researchers claim they do not. This would imply that they are phase locking with a fixed frequency offset, which is very possible. They could then design receivers which would need oniy two additional constants per satellite-a time offset and a frequency offset-in order to use either GPS and/or 
GLONASS satellites to navigate. Only circumstantial evidence of this scenario is available.

A paper by Koshelyayevskiy et al. (1984) documents time-transfer comparisons using GLONASS and portable hydrogen maser clocks over a distance of 4,000 kilometers between Mencieleyevo and Irkutsk. The first experiment they reported (August-October, 1989) showed a standard deviation on the regression line of 58 nanoseconds, and the portable clock values differed in some cases by more than 100 nanoseconds. The last experiment (July-August 1990) showed much improved results. The regression line had a greatly reduced slope, indicating they were using the technique to accomplish remote syntonization and the residuals were only 22 nanoseconds. There was only one portable clock trip for this period, and it agreed to within 15 nanoseconds.

\section{b. Television}

The Soviet state controls the timing of Soviet TV broadcast so that it is synchronous with Moscow time. Via TV timing techniques, Moscow time is synchronized to UTC(SU) with an accuracy of 20 nanoseconds. ${ }^{15}$ TV time via satellite requires an accurate ephemeris for the rebroadcasting satellite. The Soviet Union can measure the round-trip time back to the transmitter, but that only gives one of three vectors necessary for determining the position of the satellite. The Soviet footprint viewed from a geostationary satellite is large enough so that picking three stations across the Soviet Union would provide a fairly accurate satellite ephemeris. Soviet researchers report that they can communicate time, without interfering with the TV information, to all the major cities with an error of less than 500 to 1,000 nanoseconds (probably meaning time accuracy). They also report being able to provide standard frequencies at accuracies of 1 to 5 $x 10^{12}$ on a daily basis, which would suggest a time stability of about 100 to 500 nanoseconds over that same period, depending on the noise characteristics, which are not discussed (Paliy et al., 1972).

TV timing will sustain about 1 nanosecond of time stability from second to second over line-of-sight paths-improving somewhat with averaging time-

15 D. W. Allan, private conversation with scientists at VNIIFTRI, September 1989. 
but it is limited by the medium to about 100 picoseconds. The TV receivers are the limiting factor and bring both the instabilities and the inaccuracies up to about 20 nanoseconds. Soviet researchers are aware that better receivers would improve their TV timing capabilities. Such receivers have been demonstrated in the United States.

\section{c. Communication Systems}

For communication systems, the Soviet researchers would like less than 0.5-millisecond inaccuracies for the synchronization among the nodes. One paper reports a technique that synchronizes subsystems more tightly among themselves, relaxing the country-wide synchronization requirements (Barkov, 1985). The penalty for lack of synchronization is bigger computer storage buffers to avoid slippage and loss of data. For sub-networks, the Soviet goal is not more than 1 microsecond phase error accumulation over one day (Fedorov et al., 1987).

The better the clocks, the easier it is to maintain synchronization and to resynchronize. The Soviet Union is using quartz oscillators, as is the United States. These would have to be very good quartz oscillators if they can maintain 1 microsecond over a day. The Soviet literature reports the round-trip accuracy of reciprocity for radio-link paths to be 100 to 200 nanoseconds, and cable-link paths to be 300 to 500 nanoseconds for distances up to 1 megameter (Paliy et al., 1972).

\section{Terrestrial Techniques}

A recent CCIR report, entitled "Standard Frequencies and Time Signals," lists all the different transmitters, their locations, and their characteristics. ${ }^{16}$ The propagation path is the limit for these techniques, so their accuracies and time instabilities are the same as those of the United States (see Figure V.5). The reader will note in reference to the above report that the call letters for Soviet transmitters begin with the letter $\mathrm{R}$.

16 "Standard Frequencies and Time Signals," CCIR Report 267-6 (Mod F), Document 7/1008-E, (April 1989), 17th Plenary Assembly, Düsseldorf, 1990. 
Some interesting differences between the Soviet Union and the United States are worth noting for transmissions in the band from 2.5 to $15 \mathrm{MHz}$. The Soviet Union has four transmission sites (Tashkent, Irkutsk, Novosibirsk, and Moscow); the United States has two sites (Colorado and Hawaii). Tashkent and Irkutsk transmit at much lower power levels than does the United States. The power levels at Novosibirsk and Moscow are roughly the same as in the United States. The transmitters at Irkutsk and Moscow use the 4-kHz offset from the allocated frequencies, and, as mentioned before, these are the most commonly used to avoid interference (see Figure V.1). The Soviet Union does not utilize the $20-$ and $25-\mathrm{MHz}$ allocations as does the United States. The time accuracy (when the delays are accounted for) is about 1 millisecond for these Soviet transmissions. The time stability can be somewhat better, but it has very strong periodic variations due to day-night, annual, and solar-cycle ionospheric induced changes.

The 1990 CCIR report shows four Soviet transmitters in the LF band at three sites (Moscow, Irkutsk, and Novosibirsk). These stations would be nominally equivalent to the US NIST transmitter WWVB at $60 \mathrm{kHz}$. With proper theory and care, a 0.1-millisecond accuracy can be achieved using these frequencies up to distances of a few megameters. At these frequencies, there are conditions in which the ground and the ionosphere act like a waveguide, and excellent day-today phase stabilities in the order of a microsecond are achievable. These day-today frequency instabilities tend to average for longer times like flicker noise PM, and frequency transfer accuracies of a few parts in $10^{12}$ can be achieved (see Figure V.5).

The CCIR report describes a set of five transmitter sites with call letters starting with $U$, which are in the VLF band (20 to $25 \mathrm{kHz}$ ). These are located at Molodechno, Arkhangel'sk, Khabarovsk, Frunze, and Gor'kiy (Nizhniy Novgorod). These transmitters are listed as standard frequency and time signal emissions and they broadcast only a few hours each day. The frequencies used, and the band in which these broadcasts are transmitted, suggest that these transmitters could serve as backups for the US Omega navigation system. The range of these transmissions is nearly worldwide. 
There are three Loran- $C$ type transmitters in the Soviet Union; these transmitters are located at Bryansk, Syzran', and Aleksandrovsk Sakhalinskiy. These $100-\mathrm{kHz}$ pulsed carrier signals are used primarily for navigation, but as in the rest of the world, they also can provide precise timing. It is not a stand-alone timing system, because hours, minutes, and seconds are not broadcast. Once time is known from one of the standard broadcast stations to about 1 millisecond, and the correct Loran-C pulse is identified, then the time accuracy can improve to a few tens of microseconds. By identifying a particular cycle of the phase of the $100-\mathrm{kHz}$ pulse, the time accuracy can approach 1 microsecond for the groundwave signal. It is sometimes difficult to resolve the correct cycle, which can cause a discrete error of 10 microseconds. The accuracy of the sky-wave signal is only about 10 microseconds, and the individual cycles cannot be resolved reliably in this case.

The locations of the first two stations are such that two-vector ground-wave navigation could be accomplished in the western part of the Soviet Unionincluding the Moscow region. Loran-C chains usually are run in sets of three. However, the distance to the third station is too far to be included in a groundwave propagation path. The last station mentioned is located on the island just north of Hokkaido, Japan.

The reported transmitter inaccuracies imply that Soviet researchers probably use rubidium frequency standards. They may also use the sky-wave signal both for navigation and for timing to cover the remote reaches of the Soviet Union. Only a single transmitter is needed for timing. With the inaccuracies of its transmitting clocks and the distances they are covering, the Soviet Union probably does not achieve the 1-microsecond accuracy that generally is available with Loran- $C$ in the rest of the northern hemisphere.

The time stability for Loran-C type signals is a function of the averaging time. The receiver integration time constants usually are on the order of 1,000 to 2,000 seconds, and the day-to-day stability ( $\sigma_{x}[\tau=1$ day $]$ ) can be as good as about 40 to 100 nanoseconds for a ground-wave path which has a range of about 1 megameter. One-day frequency estimates are on the order of $10^{-12}$, and the frequency transfer accuracy improves with increasing averaging times to slightly better than $10^{-13}$ (see Figure V.5). 
The Soviet Union buys Western Loran-C receivers to receive non-Soviet transmissions. As in the West, these receivers probably are used for both navigation and timing. The Soviet transmitter north of Hokkaido may well be phase locked to the Japanese and even the Alaskan chain. It would be easy to tell if the signals were monitored.

Until recently (over the last two decades), Loran-C transmissions have been the best means of comparing the time and frequency of the Soviet clocks with the rest of the world. However, even for integration times of a few months, this technique is limited to about $1 \times 10^{-13}$. With cesium clock accuracies now pushing into the $10^{-14}$ domain, this technique is no longer adequate.

\section{Non-Traditional Techniques}

There are two time and frequency transfer techniques that are unique to the Soviet Union: (i) dissemination via reflection off meteor trails, and (ii) the use of hydrogen masers as portable clocks.

The meteor trail technique is based on the principle of signal reciprocity. A timing code, derived from a master clock, is transmitted on a carrier signal, typically of $72 \mathrm{MHz}$. In this frequency band, an ion cloud generated by a meteor trail will act like a mirror and bounce the signal back to Earth. A receiver detects this timing signal and transmits it back while the same meteor trail is still in place. This returned signal is received at the original sending transmitter, which also has a receiver. The round trip time can then be measured. The round-trip time divided by two is assumed to be the one-way time delay.

Using the above measurements and calculations, the remote clock's time can be determined with respect to the master clock. The uncertainties in this technique are caused by a lack of knowledge of the velocity of the ion cloud, the signal-to-noise ratio of the transmission, the dispersion of the timing signal over the transmission path, the lack of a clean definition of time on the rise time of a dispersed pulse at the receivers, the uncertainties in the transmitter and receiver equipment delays, the measurement noise, and the Sagnac effect. Soviet scientists have estimated or measured the errors caused by all of these uncertainties 
with the exception of the Sagnac effect, which is the relativistic effect due to the rotation of the Earth, and it is quite small-up to about 10 nanoseconds, depending upon the geometry of the path (Dudnik et al., 1973, 1986).

Because the Soviet Union operates meteor trail time transfer using the TV band, these operations are performed after 2 a.m. in order to avoid interference with regular TV transmissions. On a given night, Soviet researchers can detect several hundred meteor trails per hour. With each trail they can get repeat reflections, which allows them to estimate the velocity of the ion cloud. The range of a reflected signal is about 2 megameters (Dudnik et al., 1972). Both the Soviet literature and the CCDS report cite an accuracy of 100 to 200 nanoseconds. At the April 1989 CCDS, Soviet representatives reported comparing the meteor trail technique with a portable clock. They conducted the experiment over three legs of a triangle, and listed an uncertainty of 20 nanoseconds.

The time stability should be a function of the number of tracks across which the time difference between the two sites has been averaged. The literature does not indicate whether Soviet researchers are optimally processing the data they obtain. They list the equipment uncertainties at 20 nanoseconds (Dudnik et al., 1986). This 20-nanosecond number (versus the number in the earlier literature) indicates that the Soviet Union has made significant strides in the equipment, the processing, and the reduction of overall uncertainties.

Soviet scientists use the meteor trail technique to communicate time from VNIIFTRI to Pulkovo (near Leningrad). Pulkovo receives the Loran-C signal from Sylt to compare with time in the West, and to provide input into TAI and UTC. The weakest link is the Loran-C contribution.

The meteor trail technique is operationally the best mode of communicating high-precision and accurate time and frequency throughout the Soviet Union, and has been operational for some years. However, it is limited to being a pointto-point system rather than a general dissemination method because it requires a transmitter and a receiver at each point. Reducing the accuracy much below 20 nanoseconds will probably be arduous, and the natural causes of uncertaintiessuch as meteor trail dispersion-will hinder future improvement. This technique is not amenable to directly transferring frequency except through repeated 
time-transfer measurements. In the future, GLONA.SS receivers will probably replace this technique.

Another non-traditional Soviet time and frequency transfer technique in which significant work is being done involves portable hydrogen maser clocks, as discussed in Chapter III. The portable clock "Sapphire" has been taken to the Paris Observatory and to Cuba several times, where there is an ensemble of hydrogen masers.

The laboratory ambient stability of Soviet hydrogen masers is about $2 \times 10^{-14}$, which translates to a 2- to 3-nanosecond dispersion per day if one assumes a flicker noise FM model for the long-term random variations. In a typical portable clock environment, the time dispersion may be significantly increased.

In March 1991, G. A. Yelkin presented a paper updating the VNIIFTRI work on the spherical cavity hydrogen maser portable clock. Soviet researchers now have several months of operating data on this unit. It is currently configured without a cavity servo, hence, it has frequency drift. However, this can be well modeled in a portable clock post-analysis application. As discussed in Chapter III, this design has some interesting advantages, especially as it applies to portability. The spherical outer shell has a coil for servo controlling the magnetic field. With three shields and this coil servo, thr $y$ get a shielding factor of about 200,000. The most important aspect of this design is that the outer shield servo will respond to ambient magnetic field changes. This design should help minimize the time dispersion in a portable clock application.

\section{Communications Systems}

The purpose of the Consultative Committee in International Telegraphy and Telephony (CCITT) Convention is to have the frequencies agree for all communication systems within $10^{-11}$. The literature indicates that the Soviet Union is trying to conform with this standard. This number is frequency accuracy and is difficult in the long term to maintain, in an independent sense, using rubidium gas cell frequency standards. Most Western countries have chosen a hierarchy of cesium beam frequency standards, along with rubidium gas cell standards and quartz oscillator frequency standards. There is no evidence that Soviet 
researchers are using cesium beam frequency standards in any of their communication networks; they appear to use rubidium and quartz standards exclusively.

To maintain the system frequency, Soviet researchers apparently have designed their communication systems to be phase stable, but not accurate, to a microsecond. Thus, by keeping track of the phase relationship between their clocks in a network over one day, they can infer relative frequency to $10^{-11}$.

The goal of the CCITT convention is to keep the frequencies in agreement within some tolerance. As mentioned earlier, keeping the frequencies the same throughout a communications network does not require the delays to be calibrated. However, Soviet researchers sometimes appear to calibrate the delays in their microwave relay systems using reciprocity techniques. This would allow them to perform time-domain multiplexing of transmissions-a technique also being utilized in the West. Soviet synchronization of nodes appears to be very similar to that of the West (Barkov, 1985).

Synchronization throughout a communications network is needed to avoid large buffers at the receiving end and to prevent data slippages. The AT\&T synchronization goal is 10 microseconds, whereas the Soviet synchronization goal appears to be $\sim 0.5$ milliseconds (Sivov et al., 1987).

The Japanese are pushing for a new CCITT standard of 10-13 using improved commercial cesium beam frequency standards. They will meet much resistance throughout the rest of the world. However, AT\&T is installing a combination rubidium gas cell frequency standard with a GPS receiver which should be able to meet this goal except for US Department of Defense degradation of the GPS signal.

With the greatly increasing communication capacity occurring throughout the world, it is anticipated that the Soviet Union will upgrade its communication systems. Some optical fibers have already been laid, and it is likely that the published literature does not yet reflect the long-term Soviet plans. 


\section{PROJECTIONS FOR THE FUTURE}

As the GLONASS constellation reaches its full 24 satellites, it will unduubtedly become the principal Soviet means of high-accuracy time and frequency distribution. The Soviet L'nion is roughly 10 years behind the United States in this regard.

There are four principal modes in which GLONASS or GPS can be used to disseminate time or compare remote clocks:

(1) A receiver can access the time of the clock on board the satellite. Applying a correction word in the data message gives system time. The Soviet Union has made a national commitment to make that synchronous with UTC(SU), r.hich is the standard at VNIIFTRI.

(2) The satellite clocks can be used as portable clocks. As a satellite clock makes its nearest approach to a point on the ground (point $\mathrm{A}$ ), a timedifference measurement is made-accounting for the propagation delays. Later, the same satellite will make its closest approach to point $B$, and a time-difference measurement is made. Accounting for the rate differences between the clocks by successive measurements, the time difference between the clocks at points A and B can be estimated.

(3) In the common-view mode, the clocks at points A and B measure each of their time differences with respect to the same satellite during the same integration period. Then the rate and the time of the satellite clock drop out of the equation when the difference is taken between the two measurements.

(4) Over short baselines, the signals from four satellites can be received at the same time. The received information at points $A$ and $B$ at the ends of a baseline can be cross-correlated after the fact to solve for the differential position and the time difference between clocks $\mathrm{A}$ and $\mathrm{B}$.

The accuracy of the first approach, on a global basis, is less than 100 nanoseconds for GPS. The second technique has an accuracy of approximately 50 
nanoseconds. The third technique has an accuracy between 10 and 20 nanoseconds, and a time stability of between 1 and 5 nanoseconds divided by the square root of the number of days averaged. This third technique is the most cost-effective, accurate, and stable manner to compare the international timing centers. The transfer of frequency accuracy is better than the best primary frequency standards currently in existence. Therefore, this common-view technique is the de facto method for communicating clock data to the BIPM for determining TAI and UTC. The fourth method is the most accurate--better than 1 nanosecond-but it is somewhat labor intensive and only works well over short baselines.

Soviet researchers will probably pick up on all four methods for GLONASS. At the April 1989 CCDS, they agreed to install a GLONASS receiver at VNIIFTRI and to cooperate with a tracking schedule between Moscow and Leeds incorporating the common-view technique. Professor Daly at the University of Leeds has both GLONASS and GPS receivers. He has agreed to act as the transfer point to use GPS in common view to get time to the National Physics Laboratory and to BIPM. This should considerably improve the link with the Soviet Union. TAI will be able to appreciate the full accuracy and stability of the primary cesium beam frequency standards at VNIIFTRI (which now has three, and a fourth is expected to be working in the near future).

The introduction of SA into GPS will degrade the time accuracy to 167 nanoseconds, and the navigation accuracy to approximately 100 meters in two dimensions (RMS). Secure military users will be able to remove the effects of SA and maintain the same accuracy as before. On the other hand, the Soviet Union has stated that it will not introduce the equivalent of SA. This should encourage the manufacture of GLONASS receivers outside of the Soviet Union. Therefore, GLONASS could become more popular for the civil sector than GPS. A paper given at the March 1990 European Frequency and Time Forum strongly expressed the virtues of the joint use of GLONASS and GPS.17

17 C. Rosetti, "A Civil NAVSAT Overlay to GPS and GLONASS," Proc. 4th European Frequency and Time Forum, (13-15 March 1990), Keuchâtel, Switzerland. 
In general, GLONASS clocks are not as stable as GPS clocks, but the GLONASS clocks are improving in performance at a much faster rate than the GPS clocks. At the rate they are improving, GLONASS clocks will catch up with the GPS performance during the 1990s. The Soviet Union is experiencing significant operating lifetime problems with GLONASS satellites, which now typically last only a couple of years. However, GLONASS satellites are launched three at a time, and can be maneuvered into position in approximately eight days. In contrast, the United States has been launching GPS satellites one at a time. The United States is planning to launch two GPS satellites at a time, but it takes roughly six months to make a major orbital change in a GPS satellite's position.

The US Department of Transportation has signed an agreement to study the joint use of GLONASS and GPS for the Federal Aviation Administration and for the Soviet air traffic control system. Contracts have now been let for receivers which will utilize either or both systems. As discussed earlier, there are indications that Soviet researchers have already been studying the joint use of the two systems (Malyy, 1984).

Since the Soviet meteor trail time-transfer technique is operational, and a fair amount has been invested into equipment, this system can be expected to continue with some small improvements.

The Soviet Union will probably continue to utilize other traditional techniques. Given the econumic squeeze, the Soviet Union may cut back in some seemingly less fruitful areas. The Soviets are already broadcasting only part of the time on several of their transmitters. If the Soviet economy were stronger, they would undoubtedly transmit more hours per day. They would probably also install more Loran-C transmitters, as there are not enough transmitters to give good ground-wave navigation and timing across the Soviet Union. 
(blank)

V-38 


\section{CHAPTER V: TIME AND FREQUENCY TRANSFER AND DISSEMINATION REFERENCES}

Barkov, V. M., "Synchronization of Toll Digital Communications Junctions," Telecomm. Radio Eng., 39/40, 11(1985), 5-12.

Belotserkovskiy (Belotserkovskii), D. Yu., and G. N. Paliy (Palii), "State Time and Frequency Service in the Soviet Union," Meas. Tech., 15, 12(1972), 1804-1811.

Bolotnikov, M. V., and A. G. Polyak (Poliak), "Effect of Frequency on Curvature Correction in the Approximate Formula for Calculating the VLW Signal Phase Velocity," Meas. Tech., 19, 6(1976), 661-663.

Bordovitsyna, T. V., L. E. Bykova, V. A. Tamarov, and N. A. Sharkovskiy (Sharkovskii), "Structure of Perturbations in the Orbital Motion of Navigational Earth Satellites Similar to NAVSTAR," Cosmic Res., 23, 5(1985), 557-561.

Cherenkov, G. T., "Time Position of Time-Signal Checks Transmitted over the Radio-Broadcast Network," Meas. Tech., 17, 5(1974), 690.

Dudnik, B. S., B. L. Kashcheyev (Kashcheev), A. Ya. Leykin (Leikin), A. N. Smirnov, and M. D. Sopel'nikov, "Experimental Investigation of Coordination of Time Scales on Two Meteor Channels," Meas. Tech., 15, 11(1972), 1667-1670.

Dudnik, B. S., B. L. Kashcheyev (Kashcheev), and A. N. Smirnov, "Use of Reflected Radio Waves for Calibrating Tie-In Channels of Time Scales," Meas. Tech., 16, 6(1973), 349-353.

Dudnik. B. S., Yu. A. Koval', V. S. Kushch, and S. B. Pushkin, "Accuracy Criteria for Radio-Meteor Systems for the Time-Scale Synchronization," Meas. Tech., 29, 2(1986), 93-97.

Fedorov, Yu. A., V. Ya. Bol'shakov, and N. I. Yermilov (Ermilov), "Automatic Remote Control System for the Output Time of Television Frame Synchronizing Pulses," Meas. Tech., 16, 1(1973), 65-68.

Fedorov, Yu. A., Yu. D. Ivanova, and A. I. Shchavelev, "Coordinateless Method of Measuring Signal Transmission Time Through Satellite Television Channels," Meas. Tech., 30, 2(1987), 158-159.

Fleer, A. G., "Variations in Propagation Time of Short Radiowaves and Nonuniformity of Earth's Rotation," Meas. Tech., 15, 11(1972), 1675-1687.

Fleer, A. G., and A. I. Shchavelev, "Seasonal Variations in the Probability of Deep Fading on Transitional VLW Paths," Meas. Tech., 19, 5(1976), 666-668.

Fleer, A. G., and V. A. Stepanov, "Problems of Experimental Investigation of Radiowave Propagation for Time Service," Meas. Tech., 15, 11(1972), 1671-1674.

Kholshevnikov, K. V., L. L. Sokolov, Ye. I. Timoshkova, and V. B. Tivov, "Accuracy in Predicting Orbital Motion of Artificial Earth Satellite," Vestn. Leningr. Univ. Math., 4(1984), 68-71.

Koshelyayevskiy, N. B., and S. B. Pushkin, "USSR National Time Unit Keeping Over Long Intervals Using a Set of Hydrogen Masers," Proc. 22nd Precise Time and Time Interval Applications and Planning Mtg., 4-6 December 1990. 
Malyy (Malyi), M. M., "Creation and Use of a Unified Time System for Civil Aviation," Meas. Tech., 27, 5(1984), 419-421.

Matveyenko (Matveenko), L. I., "Radio Interferometry and Far Cosmic Navigation," Zemlya $i$ vselennaya, 4(1987), 20-26.

Minullin, R. G., G. N. Paliy (Palii), V. V. Sidorov, A. V. Sukhotskiy (Sukhotskii), and Yu. D. Ivanova, "Locking into the State Standard of Time by Using Reflections from Meteors," Meas. Tech., 14, 1(1971), 33-35.

Paliy (Palii), G. N., "Experimental Evaluation of the Error of Joining Time Scales over Wide-Band Communication and Television Channels," Meas. Tech., 13, 5(1970), 670-674.

Paliy (Palii), G. N., and Yu. A. Fedorov, "System for the Simultaneous Transmission of Time Signals and Information as to the Current Time Along Television Channels," Meas. Tech., 18, 1(1975), 85-87.

Paliy (Palii), G. N., and Yu. A. Fedorov, "Method of Relating Time Scales with the Aid of a Satellite Link and Terrestrial Television Networks," Meas. Tech., 15, 3(1972), 380-384.

Paliy (Palii), G. N., Yu. D. Ivanova, and S. B. Pushkin, "Prediction Error for the Transmission Time of Signals Via Satellite Television Channels," Meas. Tech., 28, 5(1985), 393-395.

Paliy (Palii), G. N., Ya. I. Luk'yanchenko (Lukianchenko), Yu. A. Fedorov, and E. M. Vnukov, "Experimental High-Precision System for Transmitting Time Units and Frequency over Television Channels," Meas. Tech., 15, 1(1972), 54-59.

Sivov, V. A., Yu. G. Pisarev, and V. I. Prytkov, "Synthesis of an Dptimal System of Clock Synchronization Using a Relay Pilot Signal," Signal Generation and Conversion, UDC 621.396.622, Telecomm. Radio Eng., 41/42, 1(1987), 28-33.

Vasin, L. D., E. S., Kiritsev, and B. M. Kireyev (Kireev), "Apparatus for Frequency Collation Using the Signals of Super-Long Wave Radio Stations," Meas. Tech., 15, 11(1972), 1656-1658. 


\section{CHAPTER VI}

\section{METROLOGY AND STATISTICAL REPRESENTATIONS}

\section{A. SUMMARY}

This chapter addresses methods of measuring time and frequency, the methods of describing the characteristics of clocks and oscillators, some useful statistical techniques, and some pertinent relativistic issues. The Soviet literature provided little information in this area, but explicitly and implicitly, several papers with non-related titles touched upon the measurement methods and statistical techniques. A review of the literature revealed no big surprises or unusual trends.

The Soviet Union has focused more energy on producing a clock with a frequency reference in the optical region of the spectrum than any other country. Soviet scientists have had some success but no breakthroughs.

V. P. Chebotayev of the Thermal Physics Institute in Novosibirsk is clearly a world leader in the area of time and frequ.ncy. Several years ago, the Soviet Union announced its goal to base the state standard for frequency on a standard built in the optical region of the spectrum. Apparently Chebotayev was the main motivator behind that goal (Chebotayev, 1978). Referring to K. Evenson's work at the US National Bureau of Standards (NBS), S. N. Bagayev made the following statemient:

This provides a basis for the establishment of a joint time and length standard right up to frequencies of $10^{16} \mathrm{~Hz}$ with the aid of lasers. This joint standard should be capable of transferring all the frequency characteristics of the most stable source to any range without loss in precision (Bagayev et al., 1982).

This goal has not heen realized, nor will it be in the near future. There is a massive effort to accomplish this goal, but it probably will never be achieved without some loss in precision. The statement reveals Soviet goals and aspirations, however, and this is an important consideration in assessing the Soviet program.

In the last few years, the Soviet Union appears to have backed off from that goal. In spite of the lack of progress made in the development of a measurement 
system to optical frequencies, Soviet scientists claim that what is needed is "a qualitatively new jump...in the years ahead." They also claim that decreasing the influence of second-order Doppler effect would allow them to get down to the limit of $10^{-16}$ or $10^{-17}$ :

Simplification $o^{f}$ the frequency synthesis scheme will permit developing optical time standards, which will be able to compete in practice with the best microwave time and frequency standards. The accurate frequency measurements in the optical range must reach values of $10^{-11}$, and the measurement technique itself must be simple and widely available (Bagayev and Chebotayev, 1986).

Otherwise, Soviet time and frequency measurement equipment appears to be quite traditional. The stability, accuracy, and measurement noise achieved by the Soviet Union are somewhat lagging those of the West. Visits to Soviet laboratories revealed some measurement equipment from outside the Soviet Union, but much Soviet-made equipment was also observed. Some of the Soviet instrumentation and concepts are well advanced. For example, Soviet researchers have an instrument which will measure time delays with an error as small as 20 picoseconds (Yefremenko et al., 1987).

The Soviet Union apparently has not implemented the dual-mixer timedifference technique, which has become an important measurement tool in the 'Vest. ${ }^{1}$ China, along with several other laboratories throughout the world, has also picked up this technique. This technique is cost effective and can provide sub-picosecond stabilities from second to second and accuracies of a few picoseconds.

In terms of time and frequency statistical processing techniques, outside of a few very clever schemes, the Soviet Union has mostly converged to using the same measures that have become standard in the rest of the world. For example, Soviet scientists have been using the Allan standard deviation for characterizing the stability of their clocks for roughly the last decade. However, they apparently are not aware of the modified Allan deviation. One clever scheme they have developed is drift estimation using a Kalman filter, and, in this regard, they seem to be leading the United States (Borisov, 1984).

1 For example, see D. W. Allan and H. Daams, "Picosecond Time Difference Measurement System," Proc. 29th Annual Symp. on Frequency Control, (1975), 404-411. 
For measuring clocks remote from each other and in the vicinity of the Earth at relativistic levels of accuracy, the Soviet Union seems to have followed the conventions developed in the West. ${ }^{2}$

\section{B. INTRODUCTION}

Some of the basics of clock measurement are outlined in the beginning of this report. Because the measurement of time interval is the most accurate measurement in metrology, some special techniques have evolved. How do you measure 16 significant digits of frequency, for example? Or how do you measure time stabilities in the sub-picosecond range? How do you measure the time difference of clocks remote from each other to an accuracy of a nanosecond? These questions have been asked, and answers are at hand in state-of-the-art measurement systems. The inclusion of relativistic terms becomes an engineering necessity when operating in the coordinate frame of the spinning Earth.

One of the secrets in conducting high-precision metrology is to set up a measurement system or an experiment to achieve a null result or a near-null result. A classic example for making frequency measurements is the common heterodyne technique. This technique provides a measurement via a frequency mixer by generating a signal at the difference in the frequency between two input frequencies. The key elements in the frequency mixer are a non-linear Schottcky barrier diode, which has extremely low noise, and a low-noise, narrow-band filter. If the frequencies are the same, the difference is zero. If there is a slight difference, the beat or difference frequency is easily measured. Suppose, for example, that a $10.000000 \mathrm{MHz}$ signal and a $10.000001 \mathrm{MHz}$ signal are the inputs. In this case, the beat will be $1 \mathrm{~Hz}$. If one of the input signals changes only 0.1 part per million, then the beat will change by $1 \mathrm{~Hz}$, which is a 100 percent change. Therefore, small changes in a carrier are magnified by the heterodyne factor-in this case 10 million. This heterodyne measurement technique can be used for frequencies up to the optical region of the spectrum. The non-linear mixing elements may change, but this beat-frequency measurement technique is

2 See N. Ashby and D. W. Allan, "Practical Implications of Relativity for a Global Coordinate Time Scale," Radio Science, 14, 4(1979), 649-669. 
employed by time and frequency metrologists throughout the world, including the Soviet Union. In fact, the Soviets probably have developed some of the best optical frequency standards, and their assessment of quality uses this beat-frequency measurement technique.

For time-difference measurements, a wide bandwidth time-interva! counter is typically used. This device measures the time interval between a start pulse from one clock and a similar pulse to stop the counter from another clock. For example, these pulses can be obtained by dividing $10 \mathrm{MHz}$ by 10 million to generate one pulse per second from a fast gate divider. The $10-\mathrm{MHz}$ signal has its own instability, which may be degraded by the noise from the frequency divider and the time-interval counter. The best counters in the West have an accuracy of $\sim 100$ picoseconds, and a time stability of $\sim 20$ picoseconds for sample rates in the vicinity of 1 second.

The time-interval counter used in the Soviet State Time and Frequency Standard at VNIIFTRI (the Physical-Technical and Radio Engineering Measurements All-Union Scientific Research Institute) has a resolution of 1 nanosecond, which would give it an RMS second-to-second stability of $\sim 0.3$ nanoseconds. Soviet scientists claim an accuracy of about 1 nanosecond for this measurement system.

The "Quartz" Scientific Production Association (NPO) in Gor'kiy ${ }^{3}$ is now marketing a frequency comparator-the Ch7-45-which has outstanding performance. Its measurement error for 1 second integration time is 1 part in 1013, and for one hour its measurement error is 1 part in $10^{15}$ when measuring at $5 \mathrm{MHz}$. The basic operating frequency of this comparator is at $100 \mathrm{MHz}$. If the measurements are made at $100 \mathrm{MHz}$, then the heterodyne factor is improved by 20. Time differences with a precision of 1 picosecond also can be measured with a different version of this device, however, there is no mention of its accuracy in the literature. This frequency comparator is integrated into the hardware package of the Ch1-75 hydrogen maser, and was included in the Ch1-75 hydrogen

3 The city of Gor'kiy has recently resumed its pre-1932 name, Nizhniy Novgorod. As the research for this report predates this recent change, the Gor'kiy name will be retained throughout the report. 
maser which was brought to the Smithsonian Astrophysical Observatory for performance comparisons in November 1990.

Another useful frequency measurement technique is to multiply the carrier frequency by some integer to some higher frequency. The deviations are multiplied by the factor of multiplication. By multiplying and differencing repeatedly, the measurement frequencies can be kept low. The deviations will continue to expand until they become easy to measure, even if they are minuscule. This technique is used at VNIIFTRI for making frequency (not time) measurements between hydrogen masers and primary cesium beam frequency standards.

Table VI.1 presents a summary of the different types of measurement systems and their stabilities and accuracies.

\section{SOVIET TIME AND FREQUENCY MEASUREMENT SYSTEMS}

Relatively few published Soviet papers deal directly with time and frequency measurement systems; however, in general, the techniques discussed in the introduction to this chapter seem to be commonly used in the Soviet Union.

A paper, entitled "Improving the Accuracy of Time Interval Measurements by the Random Coincidence Method," by Tyrsa and Khokryakov (1987) discusses a specialized technique that is applicable when the initial phase of the measured pulse is random and uniformly distributed, as would be the case due to the frontend gating (or trigger noise) in a time-interval counter. This technique appears to be similar to one developed at the Physikalisch-Tecknische Bundesanstalt (PTB) in Braunschweig, (West) Germany more than 20 years ago. The net effect is to increase the precision one more significant digit than that which is available from the time-interval counter readout. The specific accuracy and stability capacity of this technique is not cited in the paper. 


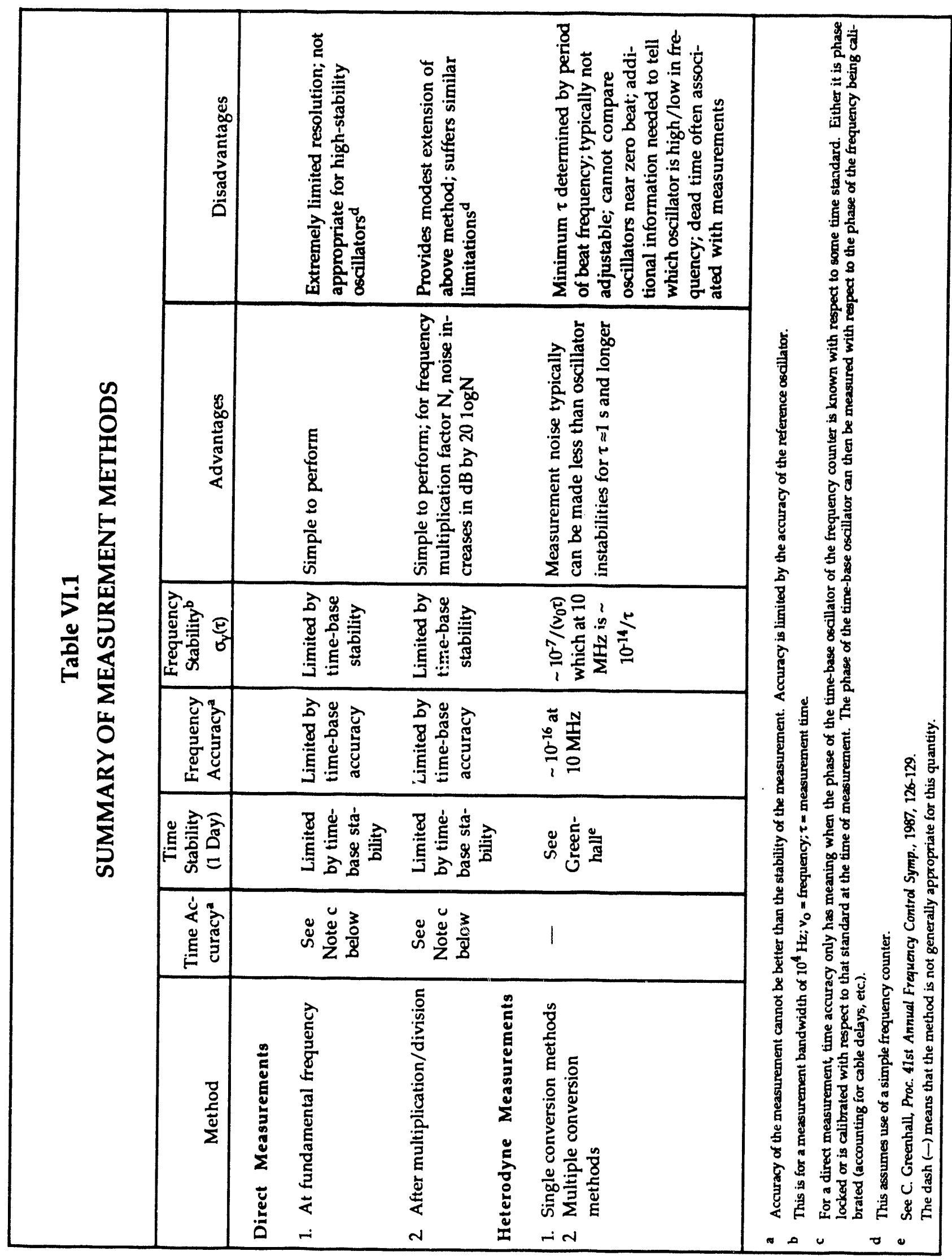




\begin{tabular}{|c|c|c|c|c|c|c|}
\hline \multirow{7}{*}{ 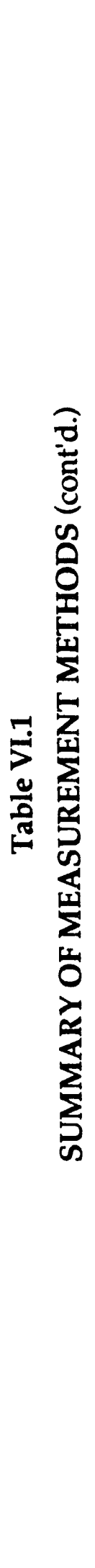 } & 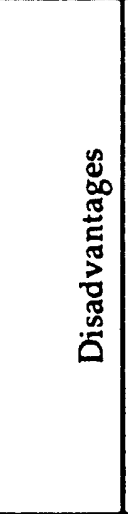 & 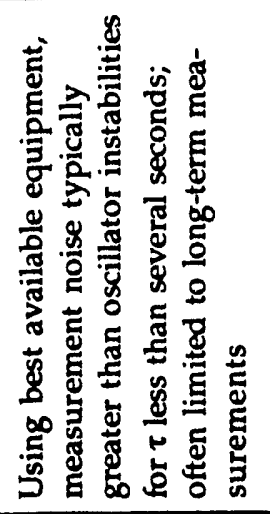 & 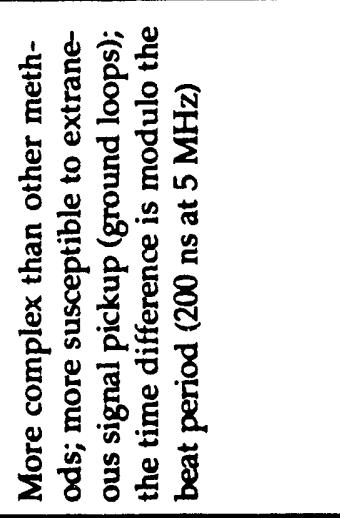 & 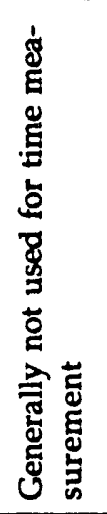 & 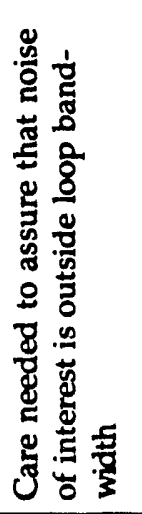 & 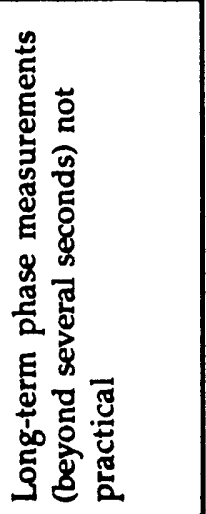 \\
\hline & 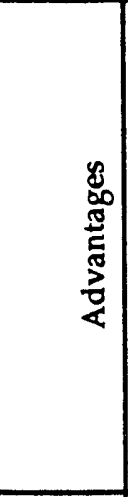 & 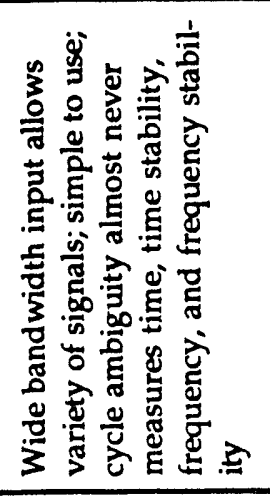 & 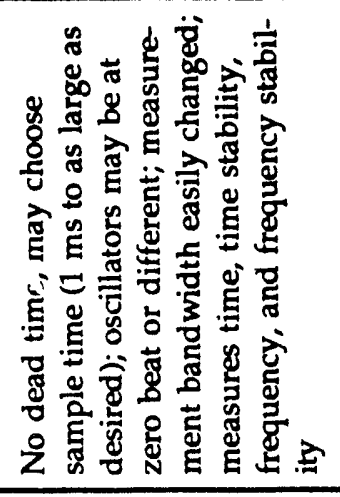 & 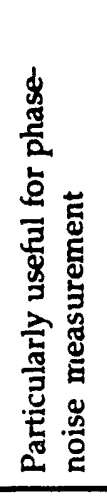 & 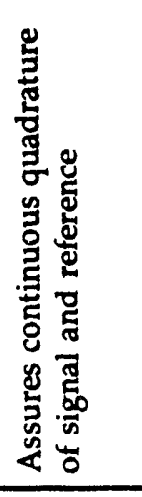 & 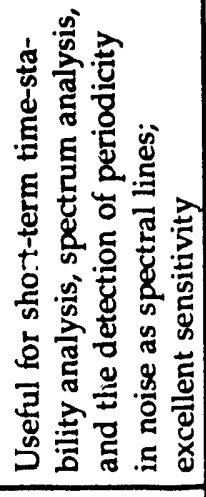 \\
\hline & 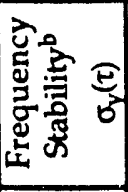 & 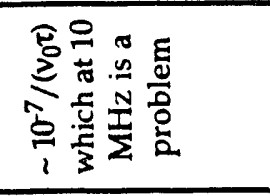 & 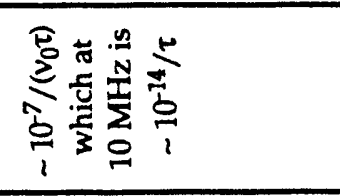 & 1 & 1 & 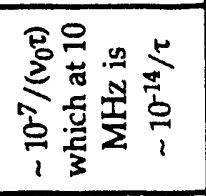 \\
\hline & 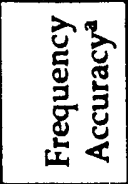 & 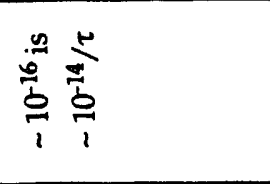 & 草 & 1 & 1 & 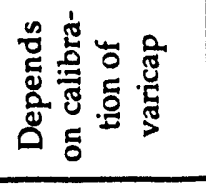 \\
\hline & 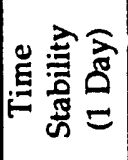 & 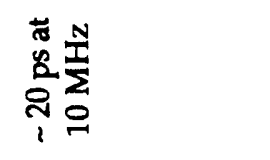 & $\frac{4}{2}$ & 1 & 1 & 1 \\
\hline & 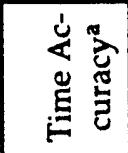 & $\frac{n}{8}$ & $\stackrel{4}{2}$ & 1 & 1 & 1 \\
\hline & 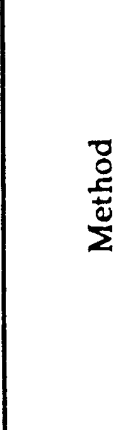 & 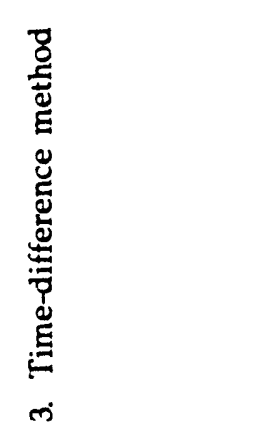 & 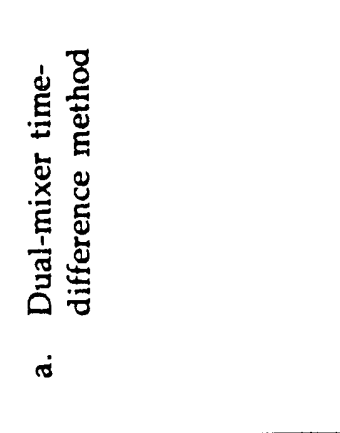 & 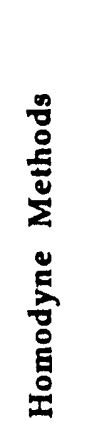 & 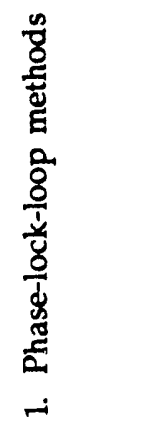 & 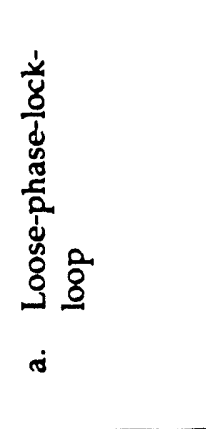 \\
\hline
\end{tabular}




\begin{tabular}{|c|c|c|c|c|c|}
\hline \multirow{7}{*}{ 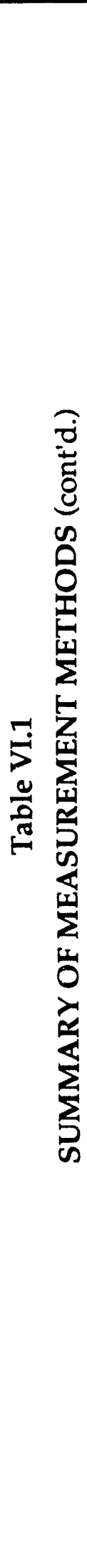 } & 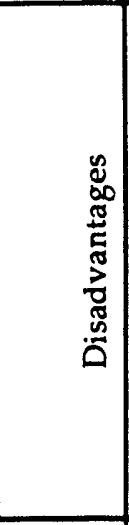 & 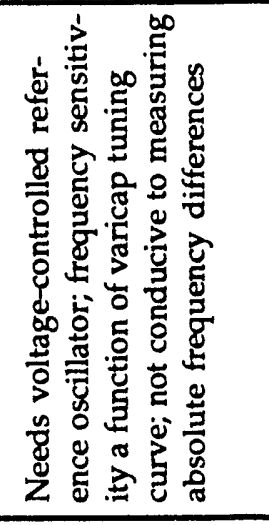 & 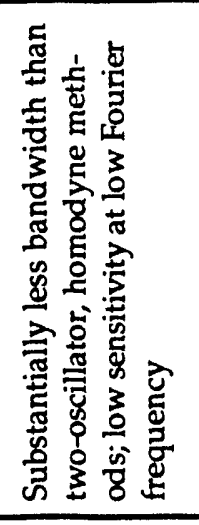 & 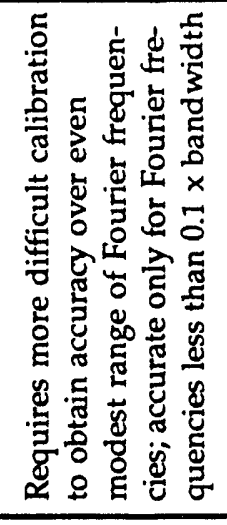 & 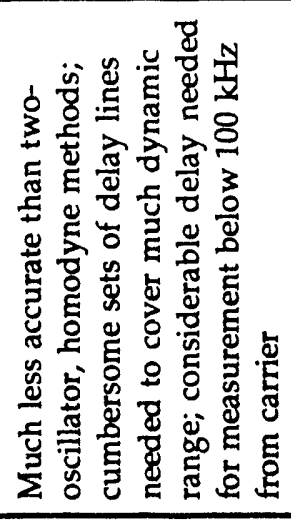 \\
\hline & 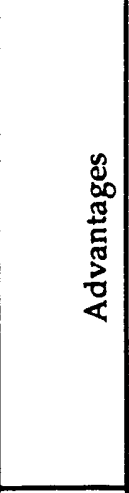 & 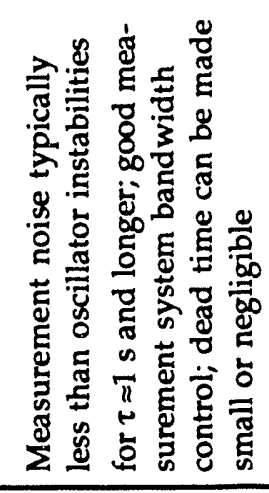 & 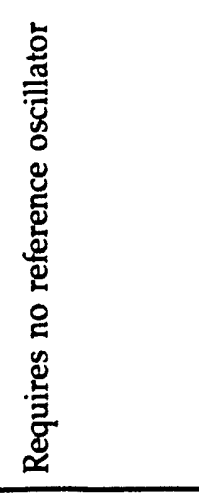 & 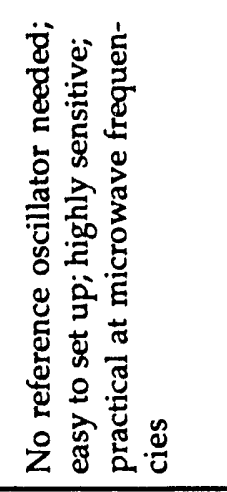 & 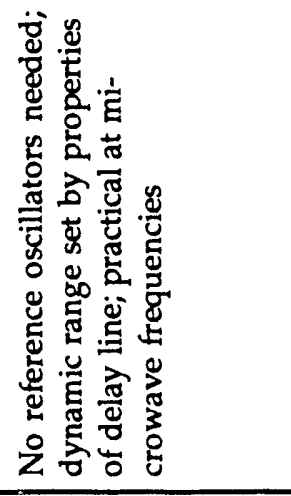 \\
\hline & 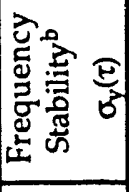 & 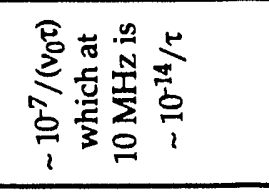 & 1 & 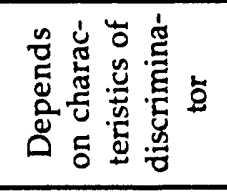 & 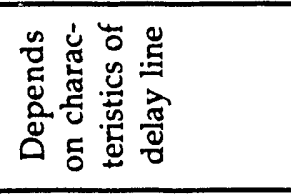 \\
\hline & 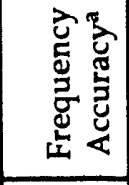 & 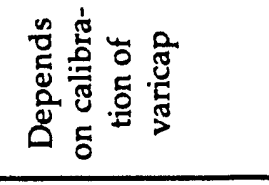 & 1 & 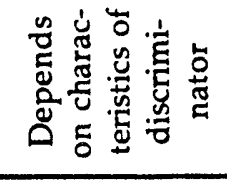 & 1 \\
\hline & 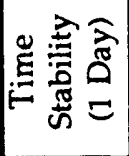 & 1 & 1 & 1 & 1 \\
\hline & 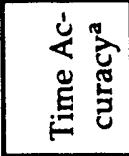 & 1 & 1 & 1 & 1 \\
\hline & 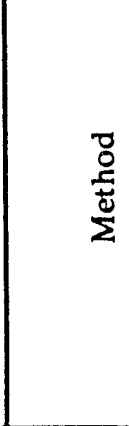 & 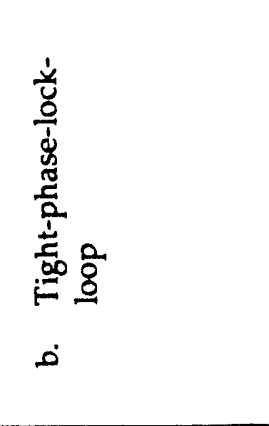 & 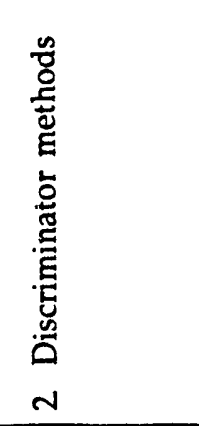 & 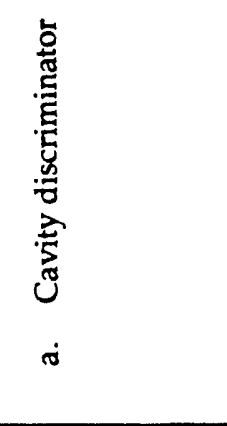 & 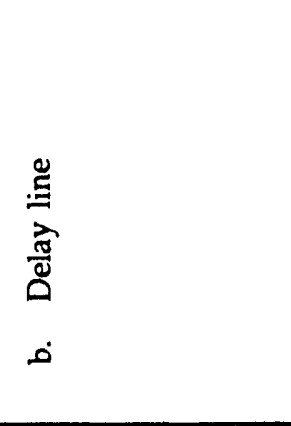 \\
\hline
\end{tabular}


V. A. Logachev and G. P. Pashev (1983) describe an interesting statistical process to determine the frequency drift in a near cptimum way. They present a simple computing method which yields results that are roughly 80 percent of the optimum for drift estimations taken in the presence of white-noise FM, flicker noise FM, and random walk noise FM.

An excellent paper by Borisov (1984) discusses the determination of longterm stability of quantum frequency standards by Kalman filtering. The Soviet Union is leading the United States in this area. Some US work has been conducted on modeling processes with flicker noise using Kalman filtering. Borisov indicates that extremely good estimates of frequency drift can be made using the Kalman-Bucy filter for both the frequency drift term and the levels of stochastic processes. Drift estimates are precise to 1 percent with 400 readings. Given such an estimate of drift, he could then measure the stochastic levels at longer time intervals, using the Allan deviation of the residuals. This process gave a clearer characterizition of clock performance in terms of the long-term stability and predictability of its oscillator. This paper is also relevant to the GLONASS effort because the clocks on board the satellites have frequency drift in the presence of flicker noise. Optimum time prediction in the presence of frequency drift is dependent on proper modeling. It appears that the Soviet Union has developed an effective tool in this regard-a tool that could be used for GPS.

D. A. Yefremenko and coworkers appear to have developed an outstanding vector volt meter (Yefremenko et al., 1987). They report data on calibrating coaxial line lengths of roughly 8 to 14 centimeters with a precision of \pm .33 picoseconds. They claim a resolving power of 3 picoseconds over a measurable delay range from 20 picoseconds to 100 microseconds with a reiaive error of 3 parts in $10^{6}$. The range of pulse string periods is from 100 to a million nanoseconds with a step size of 20 nanoseconds. This time-position meter has a resolving power of roughly 3 picoseconds, and a measurable delay range from 20 picoseconds to 100,000 microseconds with a relative error of 3 parts per million. It provides meteorological characteristics over wide signal-parameter ranges, as well as error histograms and distribution moments. This device appears to have state-of-the-art speed logic and is competitive with the West. 
Visits to VNIIFTRI revealed none of the above exceptional techniques. As mentioned earlier, Soviet scientists were using a standard 1-nanosecond timeinterval counter, making measurements every hour of all the masers against each other and against their primary cesium beam frequency standards. The problem with this approach is that the measurement noise is significantly above the clock noise at integration times of one hour. Soviet scientists would be better off to measure once a day or to average their measurements, then the measurement noise would be less than the clock noise. When this was pointed out to them, they seemed to be aware of the problem. The Soviet measurement approach for generating the official UTC(SU) time is nearly identical to that of the US Naval Observatory (USNO), including the measurement period rate of once per hour using a time-interval counter to measure the time differences. However, the USNO time-interval counter is more than an order of magnitude better.

As GLONASS becomes more important for time and frequency dissemination and as a time reference system, the position of the satellites (ephemerides) will become more important. Thirty centimeters of delay uncertainty amounts to 1 nanosecond of time transfer uncertainty. Bordovitsyna et al. (1985) have conducted a theoretical study of the US NAVSTAR GPS system and appear to be leading US researchers in the orbital mechanics involved. Soviet scientists are good orbital mechanicians and are probably doing an excellent job with the orbital calculations for the GLONASS system, although this has not been confirmed from GLONASS experimental data taken in the West. It may be that their theoretical work and their operational procedures for GLONASS orbit determination are not together.

The Bordovitsyna paper states that the Soviet Union plans to configure 24 satellites in three orbital planes at 63.4-degree inclination to the ecliptic with eccentricities of 0.01 or less. High-speed, motion-prediction computer algorithms operating with 16-bit words provide more than 1 millimeter of error over 60 orbital revolutions. The authors estimate that the spatial position of a NAVSTAR-type satellite should not exceed 100 meters for every 60 revolutions of the satellite with a good determination of the coefficients of the gravitational field. They perform an error analysis of the various perturbing effects and cite 
that the most significant effect is radiation pressure. They apparently are devising accurate, high-speed algorithms for predicting satellite motions.

No Western literature is cited in the bibliography of the Bordovitsyna paper. Bomberg is the relativist of the group and he attended the August 1988 International Astronomical Union (IAU) meeting in Baltimore. He is on a relativity committee for the IAU to determine the proper use of relativity in astrometry, and is highly competent.

A paper by Kholshevnikov et al. (1984) describes the use of laser systems to measure satellite positions from 1 centimeter to 1 meter. They report the perturbing effects that must be taken into account and discuss the most important of the following perturbations:

- perturbations from geopotential;

- lunar solar and planetary perturbations;

- atmospheric drag;

- solar and terrestrial radiation pressure;

- perturbations related to coordinate system, such as precession, nutation, motion of the poles, and continental drift;

- relativistic effects;

- electromagnetic forces (charged satellite in magnetic field);

- finite dimensions of artificial satellite;

- influence of asphericity of artificial satellite on orbital motion; and

- errors in coordinates of tracking station.

Their complete list is cited above to show the thoroughness of the Soviet analysis. No other group in the world has even approached 1-centimeter accuracy for orbital determination. In theory, they appear to be better than any other group.

For improved prediction accuracy, the problem becomes increasingly complex. Soviet scientists predict that an accuracy of 1 meter is attainable. However, an accuracy of 1 centimeter apparently cannot be attained without invoking fundamentally new ideas-the solutions being intractable with current theory and computer technology. 
Based on a preliminary study conducted by Decker and others in conjunction with time transfe: with the space shuttle, it is estimated that the United States could realize about 10 centimeters with a hydrogen maser on board and a laser retro-reflector-in addition to Vessot's Doppler cancellation system and the three-link Doppler and ionospheric cancellation system. 4

There was no direct information on how Soviet researchers determined the ephemerides for GLONASS. Apparently there are two tracking stations-one of which is near Moscow - and there may be a third station nearing completion. Soviet scientists at VNIIFTRI stated that they thought a cross-link ranging system was operating on the GLONASS satellites, however, other Soviet scientists have disputed this claim. Cross-link ranging would allow determination of the distance between the spacecraft and would be a tremendous advantage in making geometrical solutions. Cross-link ranging is planned for the GPS Block $2 \mathrm{R}$ configuration in the 1995-1996 timeframe.

If GLONASS has implemented the cross-link ranging system, then only one tracking station is necessary-rather than the five the United States must maintain for GPS. Also, cross linking reduces the demands of the onboard clock stability. If the actual numbers come close to the theoretical numbers, GLONASS could be a much better time transfer system than GPS-even when selective availability (SA) is not used. Observations made by Professor Daly at the University of Leeds indicate that GLONASS navigational accuracy presently is not as good as that of GPS with SA turned off.

\section{PROJECTIONS FOR THE FUTURE}

The quality of time and frequency metrology depends on the instrumentation, techniques, signal-to-noise ratios, methods, and theories of processing, as well as the quality of the clocks involved. Each of these elements as it relates to the future plans of the Soviet Union should be considered.

4 R. F. C. Vessot, and M. W. Levine, "Performance Data of Space and Ground Hydrogen Masers and Ionospheric Studies for High-Accuracy Frequency Comparison Between Space and Ground Clocks," Proc. 28th Annual Symp. on Frequency Control, (1974), 408-414. 
Generally, Soviet time and frequency instrumentation is not as advanced as that of the West (including Japan), with perhaps a few notable exceptions: (i) a vector voltmeter-like device used for measuring electrical lengths with precisions of 3 parts per million (as good as 20 picoseconds); (ii) a Soviet frequency comparator capable of 1-picosecond precision in measuring time difference, and a precision of 0.1 picosecond $/ \tau$ for frequency measurements over intervals, $\tau$; and (iii) the Soviet ability to phase lock a frequency divider chain to an optical atomic frequency reference. Although Soviet researchers have excelled in making an optical clock, they admit that their system for doing this is so cumbersome that it is impractical, and that a major breakthrough is needed before an optical transition will be a useful clock. The Soviet Union, along with the rest of the world, is actively pursuing this possibility. If commerce opens up between the Soviet Union and the rest of the world, the Soviet acquisition of time and frequency measurement instrumentation clearly will be advantageous to the Soviet Union and slightly advantageous to the West.

Soviet laboratory techniques are not exceptional; most of their techniques can be found outside the Soviet Union. There are some techniques available abroad which Soviet researchers could utilize. In particular, they have never utilized the dual-mixer time-difference system developed in the West, whereas China has. If commerce opens up, Soviet researchers can be expected to acquire some of the Western techniques.

The Soviet Union is lagging the West in using satellite techniques for measuring clocks that are remote from each other. It is on a par with the West in most traditional terrestrial techniques; however, Soviet scientists have performed some exceptional modeling of the effects of the ionosphere on propagation delay of timing signals. This modeling could help improve both their timing and navigation accuracy when using the VLF band. This modeling could also help them use, for example, the US Omega system. The Soviet Union clearly is leading the rest of the world in utilizing reflections off meteor trails for comparing clocks that are remote from each other. Future Soviet advances with this technique are limited by the basic behavior of the reflecting ion cloud left by the meteorites as well as by signal-to-noise and instrumental considerations. The accuracy limit appears to be roughly 20 nanoseconds. The West has alternative techniques, which are better. 
The Soviet Union undoubtedly will continue to develop satellite timing techniques. With the development of GLONASS, there are numerous ways Soviet researchers could use this system to enhance time and frequency metrology throughout their country. The GPS systems developed in the West are significantly superior to the Soviet meteor trail technique. The Soviet Union will probably incorporate these techniques into GLONASS as receivers become available. The rest of the world may turn from GPS to GLONASS if GPS SA becomes too objectionable.

GPS SA limits the non-secure receiver user to 100 -meter navigation accuracy in two dimensions, and 176 nanoseconds of timing accuracy. These accuracies are significantly worse than those of GLONASS. No information was found in the published literature that indicates whether the GLONASS system has SA capability. Soviet scientists have stated that GLONASS will not have SA capability. Since they have copied GPS in so many other important aspects, not having SA capability in GLONASS would be an unusual exception. There are active efforts in the United States to provide useful "workarounds" to deal with SA in GPS. If these are successful, there would be less motivation for the West to turn to GLONASS.

The two-way satellite timing technique apparently has not been developed in the Soviet Union. It has the potential for 1-nanosecond accuracy and time stabilities of about 20 picoseconds for integration times of about five minutes. The Soviets will probably want to participate with other countries in making time comparisons, as China has, and they will want to develop this technique within their own country. One technique currently being developed in the West is broadcasting time and frequency from a satellite. Undoubtedly, the Soviets will also take advantage of this technique.

Regarding the signal-to-noise problem, there are some subtleties here. Often, one may think all that is required to circumvent this problem is simply to increase signal power, and this is true if the noise is random and uncorrelated (white noise). However, measurement systems often can have low-frequency components-even components that have non-stationary behavior. In this case, averaging the noise may not help, especially if a mean value does not exist or if 
averaging the noise does not bring about convergence to a well-b haved value. This is often the case in time and frequency metrology-and does not seem to be generally recognized in the Soviet Union. (Soviet researchers are not alone in not recognizing these problems.) In addition to random noise, which may or may not be stationary, there are often systematic biases in measurement equipment. These biases are often determinable, and efforts generally are made to reduce these biassing effects.

An interesting signal-to-noise issue involves the measurement of millisecond pulsars. The Soviet Union, along with several other countries, has expressed a desire to participate in these exciting measurements, and Japan has launched a major campaign to participate as well. This area of metrology appears to be a race between the quality of Earth atomic timekeeping and the astrometric improvement of the signal to noise as received from the brightest millisecond pulsars. Currently, within the United States, the largest spherical radio telescope in the world at Arecibo, Puerto Rico, with a diameter of $\mathbf{3 0 0}$ meters, still has a measurement noise as large as 350 nanoseconds. This high level of measurement noise requires an integration time of about one year to achieve a performance level comparable to the best atomic clocks on Earth. Despite the large antenna size, low-noise $\left(7^{\circ} \mathrm{K}\right)$ temperature, and the sophisticated processing methods, the integrated interstellar medium perturbations across the 13,000 light-year distance cause a high level of measurement noise. The Soviet Union plans to build a bigger and better telescope than that at Arecibo. It also plans to launch two space telescopes to eliminate the influence of the Earth's atmosphere. Soviet reports claim a ten-fold improvement in signal to noise over currently available Earth-bound steerable-parabolic antennas. To achieve this goal, Soviet scientists state that they need much better short-term stability than that which is currently available from the best hydrogen maser clocks. For signal-to-noise reasons, hydrogen masers are currently the most popular radioastrometry reference clock.

As for methods and theories for processing time and frequency measurements, the Soviet Union has some fine theoreticians who seem to be doing good work in this area. A recent visit to VNIIFTRI (September 1989) revealed that the Soviets had not yet started to use the modified Allan variance, but they were very interested in doing so. They also do not appear to be using time-scale algo- 
rithms as a technique for combining the readings for optimum usage of the clocks in their ensemble for the generation of UTC(SU). As previously mentioned, Soviet scientists have developed a technique for estimating the frequency drift of a clock that is perturbed by flicker noise FM, which appears to be superior to that of the United States. If the Soviet Union has not already used this processing technique in GLONASS, it certainly would be wise to do so in the future.

Regarding better clocks for time and frequency metrology, Soviet researchers plan to launch hydrogen masers as the onboard clocks for GLONASS. They claim that they are currently launching cesium beam frequency standards-a conclusion the panel had come to by statistically analyzing the data received by Professor Peter Daly at the University of Leeds. ${ }^{5}$ In this regard, hydrogen maser reliability has been a serious Soviet issue. The Soviets cannot consistently keep their ground hydrogen masers running for long periods and, if not for their capability to launch three spacecraft at a time, they would fall behind in their GLONASS population of viable spacecraft.

In theory, with hydrogen mas ors on board GLONASS, with cross link, and with the outstanding theoretical work of Soviet orbital mechanicians, GLONASS, as a time and frequency reference system, could move ahead of GPS even without SA (intentional signal degradation). With proper receiver development, processing techniques, and high-quality clocks in the satellites and on the ground, a system such as GPS or GLONASS is capable of transferring time with an accuracy of less than 1 nanosecond and with time stabilities into the picosecond region from day to day. Soviet researchers could follow the lead of AT\&T Bell Laboratories and use a combination of rubidium clocks and GLONASS receivers at their communication network nodes and greatly improve the synchronization and syntonization of these network nodes.

The Soviet Union will continue to pursue time and frequency metrology in the optical region of the spectrum. It has been successful in this area and has some excellent scientists involved in this area of research and development.

5 P. Daly, D. W. Allan, I. D. Ketching, and T. K. Peppler, "Frequency and Time Stability of GPS and GLONASS Clocks," Proc. 44th Annual Symp. on Frequency Control, Baltimore, Maryland, (23-26 May 1990), 127-139. 
The Soviets have been among the world leaders in optical time and frequency metrology, and their goals at VNIIFTRI appear to be very much in this direction.

What is theoretically possible and what the Soviet economy will sustain may be very different scenarios. Given the current degradation of the Soviet economy, it is difficult to predict. Researchers in the United States have already learned a great deal in 1990 from direct interactions with their Soviet counterparts, and as they continue to analyze the performance of GLONASS clocks. The November-December 1990 visit by Soviet researchers to the Smithsonian Astrophysical Observatory with their hydrogen masers was a notable breakthrough of the Glasnost' era. 
(blank)

VI-18 


\section{CHAPTER VI: METROLOGY AND STATISTICAL REPRESENTATIONS REFERENCES}

Bagayev (Bagaev), S. N., V. G. Gol'dort, A. S. Dychkov, V. M. Klement'yev (Klement'ev, Klementiev), Yu. A. Matyugin (Matiugin), M. V. Nitikin, Yu. Ya. Pecherskiy (Pecherskii), and V. P. Chebotayev (Chebotaev), "Joint Time and Length Standard," Sov. J. Quantum Electron., 12, 3(1982), 267-273.

Bagayev (Bagaev), S. N., and V. P. Chebotayev (Chebotaev), "Laser Frequency Standards," Sov. Phys.-Usp., 29, 1(1986), 82-103.

Bordovitsyna, T. V., L. E. Bykova, V. A. Tamarov, and N. A. Sharkovskiy (Sharkovskii), "Structure of Perturbations in the Orbital Motion of Navigational Earth Satellites Similar to NAVSTAR," Cosmic Res., 23, 5(1985), 557-561.

Borisov, B. D., "Determination of Long-Term Stability of Quantum Frequency Standards by Kalman Filtering," Sov. J. Quantum Electron., 14, 6(1984), 875-877.

Borisova, L. B., and V. N. Mel'nikov, "Relativistic Corrections to Readings From a Portable Clock," Meas. Tech., 31, 4(1988), 323-327.

Borisova, L. B., K. A. Bronnikov, and V. N. Mel'nikov, "Taking Into Account Gravitational and Relativistic Effects in Maintaining a Unified Time Scale on Earth and Surrounding Space," Meas. Tech., 31, 5(1988), 450-455.

Chebotayev (Chebotaev), V. P., "Use of Ultra-Narrow Resonance in Spectroscopy and for Stabilizing Gas Laser Frequencies," Sov. J. Quantum Electron., 8, 9(1978), 1130-1135.

Il'in, V. G., V. V. Sazhin, M. V. Bolotnikov, and G. A. Yelkin (Elkin), "High-Precision Time and Frequency Measurements in the National Economy and Scientific Investigations," Meas. Tech., 17, 7(1974), 1033-1037.

Ivanov, V. V., and B. M. Stepanov, "Measurement of Small-Duration Intervals," Meas. Tech., 14, 1(1971), 36-40.

Kholshevnikov, K. V., L. L. Sokolov, Y. I. Timoshkova, and V. B. Tivov, "Accuracy in Predicting Orbital Motion of Artificial Earth Satellite," Vestn. Leningr. Univ. Math, 4(1984), 68-71.

Logachev, V. G., and G. P. Pashev, "Comparison of Methods of Measuring Systematic Linear Frequency Variation of Time- and Frequency-Standard Signals," Meas. Tech., 26, 10(1983), 838-841.

Logunov, A. A., and Yu. M. Loskutov, "Contradictory Character of General Relativity. The Relativistic Theory of Gravitation," Theor. Math. Phys., 67, 2(1985), 425-433.

Logunov, A. A., and Yu. M. Loskutov, "Delay of Radio Signals in the Relativistic Theory of Gravitation," Sov. Phys.-Dokl., 30, 11(1985), 971-972.

Tyrsa, V. E., and M. V. Khokryakov (Khokriakov), "Improving the Accuracy of Time Interval Measurements by the Random Coincidence Method," Meas. Tech., 30, 3(1987), 263-265. 
Yefremenko (Efremenko), D. A., I. A. Malevich, and E. I. Tabachnik, "Computerized Apparatus for Examining and Certifying Devices for Determining the Time Parameters of Measurement-System Signals," Meas. Tech., 30, 3(1987), 225-228.

\section{ADDITIONAL WORKS CONSULTED}

Abashev, Yu. G., V. N. Baryshev, G. A. Yelkin (Elkin), Yu. V. Timofeyev (Timofeev), L. I. Polyakova (Poliakova), S. B. Pushkin, V. Yu. Runov, and A. A. Ul'yanov (Ulianov), "Metrological Analysis of Type MTs-3 Cesium Frequency Reference," Meas. Tech., 29, 2(1986), 97-98.

Basov, N. G., M. A. Gubin, V. V. Nikitin, A. V. Nikul'shin, Ye. D. Protsenko, D. A. Tyurikov (Tiurikov), and A. S. Shelkovnikov, "Transportable Optical Frequency Standard and Results of Its Metrological Tests," Sov. J. Quantum Electron., 17, 4(1987), 545-546.

Belotserkovskiy (Belotserkovskii), D. Yu., V. G. Il'in, and I. V. Stepanova, "International Cooperation in the Field of Exact Time and Frequency Measurements," Meas. Tech., 18, 4(1975), 517-519.

Dubkov, V. A., E. V. Kulagin, A. I. Pikhtelev, and A. P. Sheronov, "Measurements of the Instability Characteristics of Quantum Frequency Standards," Meas. Tech., 27, 2(1984), 136-138.

Leykin (Leikin), A. Ya., and Ye. Z. Orlov, "Equipment for Comparing Frequencies of Highly Stable Generators," Meas. Tech., 16, 10(1973), 1469-1470.

Nazarov, K. I., "Certain Problems Associated with Verification of Time Measuring Equipment," Meas. Tech., 31, 4(1988), 356-358.

Polishchuk, R. F., "Time-Scale Service in the Vicinity of the Earth," Meas. Tech., 29, 8(1986), 707711. 


\section{CHAPTER VII \\ RELEVANT SCIENCE APPLICATIONS}

\section{A. INTRODUCTION}

Two areas of science stand out which require the best clocks and frequency standards. One area is relativity and gravitation and the other is very long baseline interferometry (VLBI).

Time and distance intervals form the basic elements of relativity theory which, in the case of general relativity, includes gravitation. Precision clocks thus play a key role in many tests of the theory. Relativity is also very important in the performance of the clocks themselves because in many cases the limiting effect on accuracy is the relativistic time dilation or second-order Doppler effect.

VLBI is a type of radio astronomy that, to date, has the highest capability for angular resolution. It consists of widely separated receiving sites, each requiring extremely stable oscillators. Hydrogen masers are used routinely in this application worldwide. The Soviet Union is actively developing a spaceborne VLBI radiotelescope in its Radioastron program to be used in conjunction with ground-based stations.

Quantum mechanics is an important aspect of the science relevant to precise clocks and frequency standards. While the original contributions were mainly from German, Danish, and British physicists, Soviet physicists have made strong contributions, particularly in detailed applications of quantum mechanics and measurement theory.

This chapter is divided into three sections: relativity and gravitation, astrophysics and very long baseline interferometry, and quantum measurements. 


\section{B. RELATIVITY AND GRAVITATION}

\section{Summary}

The Soviet Union has conducted a fair amount of work in relativity. In November 1965, Soviet scientists performed the very first experiment with a precision clock on a satellite; their objective was to verify its gravitational and velocity effects and to show that a clock could work well in a spacecraft. Their work in relativity theory, for the most part, is excellent. However, some of their published work on a relativistic theory of gravitation is controversial and the new theory has not yet been accepted by most experts, including many in the Soviet Union.

\section{Introduction}

The special theory of relativity states that the laws of physics are the same for all inertial systems with no gravitational fields present and moving at constant velocity with respect to each other. An inertial system is one in which free masses retain their state of uniform motion, that is, they do not accelerate. Included in this is the constancy of the speed of light; it is independent of the inertial frame in which it is measured.

The fact that two observers moving with respect to each other, both performing an experiment to measure the speed of light, get the same result may seem surprising, but this has been verified experimentally to high precision, at least for round-trip measurements. What this means is that the concept that absolute distance and absolute time are separate entities has to be given up. They are intertwined in such a way as to make the speed of light come out the same, independent of the relative velocity of the observers.

Putting this mathematically, we have, in classical Ner itonian mechanics, for absolute space and time elements, expressed in the two coordinate systems, unprimed and primed, before and after a constant velocity transformation or rotation 


$$
\begin{aligned}
d x^{\prime 2}+d y^{\prime 2}+d z^{\prime 2} & =d x^{2}+d y^{2}+d z^{2} \\
d t^{\prime} & =d t
\end{aligned}
$$

where the sum of the squares of the differential rectangular coordinate elements is the square of the differential length in the primed and unprimed coordinate system. The time differentials in the two systems are equal. This is the manner in which classical Newtonian mechanics expresses length and time invariance under constant velocity transformations or rotations.

In special relativity these are replaced by

$$
c^{2} d t^{\prime 2}-\left(d z^{2}+d y^{\prime 2}+d z^{\prime 2}\right)=c^{2} d t^{2}-\left(d x^{2}+d y^{2}+d z^{2}\right)
$$

where $c$ is the speed of light. Now the invariant quantity involves both length and time. It is easy to see that this gives $c$ for the speed of light in both coordinate systems.

The full transformation between systems moving with relative velocity $\mathrm{v}$ along the $\mathrm{x}$ axis is given by the Lorentz transformation

$$
\begin{aligned}
& x^{\prime}=\gamma(x-v t) \\
& y^{\prime}=y \\
& z^{\prime}=z \\
& t^{\prime}=\gamma\left(t-v x / c^{2}\right)
\end{aligned}
$$

where $\gamma=\left(1-v^{2} / c^{2}\right)^{-1 / 2}$. Here it is assumed that at $t=t^{\prime}=0$, the origins of the two systems coincide, and the primed coordinate system is moving in the $+x$ direction along the $x$ axis with velocity $v$. On taking differentials of both sides of all the equations, it is easy to show that Equation (2) is satisfied. This transformation reduces to the classical Galilean transformation, as it should, when $\mathrm{v} / \mathrm{c}$ goes to zero.

The Lorentz transformation that allows arbitrary velocity direction and coordinate rotations appears more complex but contains nothing new. 
Once one accepts that space and time intervals are intertwined and transform, as in Equation (3), with the resulting space-time interval invariance given in Equation (2), then it is clear that all physical vectors must transform in a similar manner or one would get absurd results. This leads to the concept of tensors and tensor transformation. The space-time vector $(x, y, z, c t)$ is a first-rank tensor, called a 4 vector. The speed of light $c$ is a rank-zero tensor, a constant. The electromagnetic field is a second-rank tensor. When one writes the laws of physics in tensor form, proper relativistic transformation is assured. This leads to fully relativistic mechanics and electrodynamics.

A little algebra on the differentials of the transformation shows that, at a fixed position in the primed coordinate system, we have $\mathrm{dt}^{\prime}=\mathrm{dt} / \gamma$, that is, the elapsed time interval indicated by a clock fixed in the primed system is less than that of the clocks in the unprimed system. Expressing the result in terms of the frequency $v$ of the clocks gives $v^{\prime}=v / \gamma$. Thus, a clock moving relative to the unprimed coordinate system with velocity $\mathrm{v}$ has a longer time interval between ticks or lower frequency than the clocks in the unprimed system, that is, it runs slow. The same result is found for a clock stationary in the unprimed system (and consequently moving in the primed system) $d t=d t^{\prime} / \gamma$ or $v=v^{\prime} / \gamma$. It runs slow or with a lower frequency compared to the clocks in the primed system. This is usually expressed as "moving clocks always run slow." This particular relativistic effect is called the second-order Doppler shift because it depends upon $\mathrm{v}^{2} / \mathrm{c}^{2}$. Another name often used for this effect is time dilation.

There is an obvious asymmetry in this effect. After all, the velocity is relative and the whole situation appears symmetric, so why should the clock that is designated as "moving" run slow? This argument has been used by some as proof that the effect must be zero. However, the apparent symmetry is removed when it is remembered that the clock that is considered as moving is, in effect, always compared against different clocks as it moves. These clocks, which have been synchronized in their own system, do not appear to be synchronized when viewed from the system containing the moving clock. This follows from the requirement that the speed of light be constant. The disagreement in synchronization produces the asymmetry. The term $\gamma v x / c^{2}$ in the fourth member of Equation (4) is the synchronization difference. 
The lack of synchronization found when clocks that have been synchronized in their own system are observed from a system moving with respect to them leads to the often used statement "the clock that is ahead is behind," where ahead refers to the first clock to go by the observer, and behind refers to its time indication.

Time dilation can be used to calculate the age difference in the twin paradox in which a traveling twin ages less than its stay-at-home sibling. However, the twins, initially together, should be brought back together again after the trip, and proper handling of the trip requires accelerated reference systems and must be treated in general relativity. Then it can be shown that the traveling twin, who experiences the forces of acceleration and deceleration, is the one who ages less.

Note that all of these relativistic time effects do not depend upon the construction of the clocks (in the twin paradox, the clock is biological). They arise strictly from the space-time interval invariance and invariance of the laws of physics in general.

A similar analysis shows that moving objects always appear to contract along the direction of motion: $L^{\prime}=L / \gamma$ where $L^{\prime}$ and $L$ are along the direction of relative velocity and $L^{\prime}$ is the apparent length of a moving rod that measured $L$ when it was stationary. A similar asymmetry occurs because of lack of simultaneity in the two systems.

These effects are small in everyday life. For example, a clock moving at $670 \mathrm{mph}\left(1 \times 10^{-6}\right.$ times the speed of light $)$ appears to run slow by about $5 \times 10^{-13}$. This speed is comparable to that of a fast jet aircraft and also the thermal speed of a fairly light atom at room temperature. A clock using a thermal beam of such atoms would thus run slow by about $5 \times 10^{-13}$, a large shift for high-quality clocks. Also, the clock would have a rate that depended upon temperature due to the velocity dependence of the atoms on temperature. Thus, one can see that not only are precision clocks useful for measuring relativistic effects, but relativistic effects also are very important in the fundamental workings of the clocks.

Additional understanding evolves from the Lorentz transformation, Equation (3). For example, it is apparent that velocities greater than $\mathrm{c}$ lead to absurd 
results, such as imaginary lengths or frequencies. It is not difficult to show that it would take an infinite amount of energy to accelerate a particle to $c$, or that velocities do not add linearly but add so that $\mathrm{v}_{\text {sum }}=\left(\mathrm{v}_{1}+\mathrm{v}_{2}\right) /\left(1+\mathrm{v}_{1} \mathrm{v}_{2} / \mathrm{c}^{2}\right)$ which can never exceed $c$ for $v_{1}$ and $v_{2}$ less than $c$. Also, it is not difficult to derive the famous relation $E=M^{2}$, the equivalence of mass and energy. Special relativity is now an everyday tool of the physicist.

When gravitation and acceleration are taken into account, the situation becomes more complex and we enter the realm of general relativity. At the heart of general relativity is the concept known as the equivalence principle. Since inertial and gravitational mass had been shown to be equal experimentally to fairly great precision, Einstein assumed they were identically equal. He then imagined doing experiments in a box initially held stationary in a gravitational field and then accelerated in a field-free environment at just the right amount and direction to duplicate the effects of the gravitational field. One cannot envision any difference in the results of experiments on masses in these two cases because of the equality of gravitational and inertial mass. Einstein then postulated that all experiments under these two conditions would give the same results. This is the simple form of the equivalence principle.

Once this is accepted, then it is possible to use any coordinate system one wishes, accelerated or not. Then one must write the laws of physics in such a way, like that in special relativity (called covariantly), that they do not depend on what coordinate system is being used. The equations become tensor equations that express the covariance.

It is not difficult to show that even a simple accelerated system, such as a coordinate system rotating about an axis, has curvature of space due to the rotation. This curvature causes the paths of free particles, as viewed in the rotating system, to be curved rather than straight, since free particles travel along what are called geodesics, the straightest possible lines in the curved space-time. Geodesics become true straight lines only in flat, uncurved space.

The curvature of the space can be related to the equivalent "gravitational" field of the rotating system leading to Coriolis and centripetal forces. The differential equations of the motion of the free particles in the curved space can easily 
be derived. Since curvature of space has the same effect as a gravitational field, real gravitational fields must also cause curvature if the equivalence principle holds. After great effort, Einstein found a tensor equation that related the curvature caused by real gravitation to the matter/energy sources. This, then, is the full essence of general relativity. It is said that "space curvature tells matter how to move and matter tells space how to curve." However, the mathematics involved in discussing this in detail are far beyond the scope of this introduction.

One very important result is the ratio of the rates or frequencies of two moving clocks at different gravitational potentials and different velocities as viewed in a frame in which all gravitating masses are stationary:

$$
\begin{aligned}
& \frac{d \tau_{1}}{d \tau_{2}}=\frac{\omega_{1}}{\omega_{2}}=\sqrt{\frac{\left(1+2 \frac{\phi_{1}}{c^{2}}-\frac{v_{1}^{2}}{c^{2}}\right)}{\left(1+2 \frac{\phi_{2}}{c^{2}}-\frac{v_{2}^{2}}{c_{2}}\right)}} \\
& \approx 1+\frac{\left(\phi_{1}-\phi_{2}\right)}{c^{2}}-\frac{\left(v_{1}^{2}-v_{2}^{2}\right)}{2 c^{2}}
\end{aligned}
$$

where $\phi_{1}$ and $\phi_{2}$ are the Newtonian gravitational potentials at clock 1 and 2 respectively, and $v_{1}$ and $v_{2}$ are the clocks' speeds. The reason for choosing the frame in which the masses are stationary is that the gravitational potentials are much more complex in a frame where there is moving matter but, of course, the results must be the same. A fairly good approximation at the Earth is a nonrotating frame with origin at the center of the Earth. The rotating mass of the Earth does have an effect, but it is extremely small.

From Equation (4) we see that a clock at an elevation above the surface of the Earth runs faster than a clock on the surface because the gravitational potential increases with height. The effect is about $1 \times 10^{-13}$ for $1 \mathrm{~km}$.

Equation (4) has been verified reasonably well to the lowest order in $\phi / \mathrm{c}^{2}$ by precision clock experiments. Tests have been proposed to look at higher-order terms, including a hydrogen maser carried close to the sun. 
From the above, one can see that precision clocks are crucial to relativity and vice versa.

\section{Discussion of Soviet Work}

The first experiment with a clock in space was performed by the Soviet Union in late 1965 (Basov et al., 1967). This was a first attempt to verify the iaws of general relativity using space technology and to perform space research with a highly stable clock. The experiment was launched on 26 November 1965 on Kosmos-97. Comparison of Earth and space clocks used the first-order Doppler canceling scheme invented in 1959 by Badessa and coworkers at the Massachusetts Institute of Technology, and was cited in Basov's references.

At the time, the experiment was a remarkable technological breakthrough. The spaceborne clock used in this experiment was an ammonia maser and its frequency could be compared to an accuracy of $1 \times 10^{-11}$ to a group of three ammonia masers on the ground. The first-order Doppler was able to be canceled to about $1 \times 10^{-5}$.

More than 10 years later, in 1976, a hydrogen maser was used in a joint NASA/SAO rocket-launched test of relativity. 1 The experiment used a more sophisticated version of the Badessa et al. system. The theoretical predictions of general relativity were tested to $70 \times 10^{-6}$ in a near-vertical flight that reached $10,000 \mathrm{~km}$.

The Soviet test was the forerunner of many future spaceborne experiments using atomic clocks. While Basov and coworkers do not claim to have tested general relativity with their pioneering experiment in an eccentric low-Earth

1 R. F. C. Vessot, M. W. Levine, E. M. Mattison, E. L. Blomberg, T. E. Hoffmann, G. U. Nystrom, B. F. Farrell, R. Decher, P. B. Eby, C. R. Baugher, J. W. Watts, D. L. Teuber, and F. D. Wills, "Tests of Relativistic Gravitation with a Spaceborne Hydrogen Maser," Phys. Rev. Lett., 45, 12(1980), 2081-2084.

R. F. C. Vessot and M. W. Levine, "A Test of the Equivalence Principle Using a Spaceborne Clock," General Relativity and Gravitation, 10, 3(1980), 181-284. 
orbit, it is clear from their paper that this was their intent. There does not appear to have been any further Soviet programs involving clocks in space until the GLONASS program.

Two papers by Logunov and Loskutov $(1985,1986)$ discussed a relativistic theory of gravitation. Their predictions differ slightly from the general theory of relativity, but these differences are sufficiently small that they are probably not detectable within the solar system. Their fundamental approach differs from that of general relativity. Most experts, including many within the Soviet Union, disagree with their interpretations and criticisms of some of the details of general relativity. For these reasons, this new theory has gained little acceptance.

Relativistic corrections to portable clocks were covered in a theoretical paper by Borisova and Mel'nikov (1988). The authors used standard general relativity and no new results were reported. Relevant papers from the West were well referenced. Soviet researchers clearly understand relativity and know how to make all of the important calculations and corrections for precision timekeeping and navigation.

A paper by Borisova et al. (1988) discussed the problems of maintaining a unified time scale on the Earth and surrounding space. Again, standard general relativity was used. It is well known that it is impossible to synchronize clocks in a rotating frame in the conventional manner usually envisioned by sending light signals back and forth. The usual solution is to synchronize the clocks to a set of fictitious co-located clocks fixed in a non-rotating frame and to make them run at the same rate as the fictitious clocks. This provides a universal time scale. All this was covered in the paper, as well as all the gravitational effects. Clocks in satellites and propagation of signals were well treated. All calculations were carried out to order $1 / c^{2}$. Again, relevant papers from the West were well referenced.

\section{Projections for the Future}

Soviet scientists are well positioned in relativity theory and practice. They know how to make all the corrections to clocks in arbitrary situations and are 
doing it in practice with their GLONASS system. They will continue to stay at the forefront in this area.

\section{ASTROPHYSICS AND VERY LONG BASELINE INTERFEROMETRY}

\section{Summary}

The Soviet Union has a VLBI network in operation and is planning to have an orbiting radio telescope in its Radioastron program which will allow VLBI to be conducted with much longer baselines in conjunction with ground stations. Soviet researchers are now collaborating with colleagues in other countries in some observations, and they plan to work with a number of other countries when Radioastron is in operation. Based on a small amount of literature and the experience of this panel, it appears that the Soviet Union has good capabilities in this area. Soviet signal processing equipment is apparently lagging that of the United States and Japan. However, Soviet scientists and engineers appear to be very capable in pursuing VLBI work.

\section{Introduction}

VLBI uses wideiy separated microwave receiving sites that have large antennas and very stable local oscillators. It works by effectively combining the signals received from a pair of antennas and looking at the interference between them. Figure VII.1 shows an idealized VLBI setup as viewed from the South Pole with two antennas at the equator of the Earth receiving a plane wave signal of wavelength $\lambda$. As the Earth rotates, the difference in phase between the two signals from the antennas is given by $\Delta \theta=B \sin ($ alpha) $/ \lambda$, where $B$ is the baseline length, $\lambda$ is the signal wavelength, and alpha is the angle between the baseline and the wavefronts. As the Earth rotates, the phase angle changes because alpha changes. The angular resolution of the interferometer is given by the rotation of the baseline necessary to change the phase difference between the two signals by about a radian and is on the order of $\lambda / B$. For $B=7,000 \mathrm{~km}$ and $\lambda=18 \mathrm{~cm}$ (the hydroxyl line), the resolution is roughly $5 \times 10^{-3}$ seconds of arc. Using shorter wavelength and/or longer baseline improves the resolution. To date, the highest angular resolution of all types of astronomy has been obtained with VLBI. 


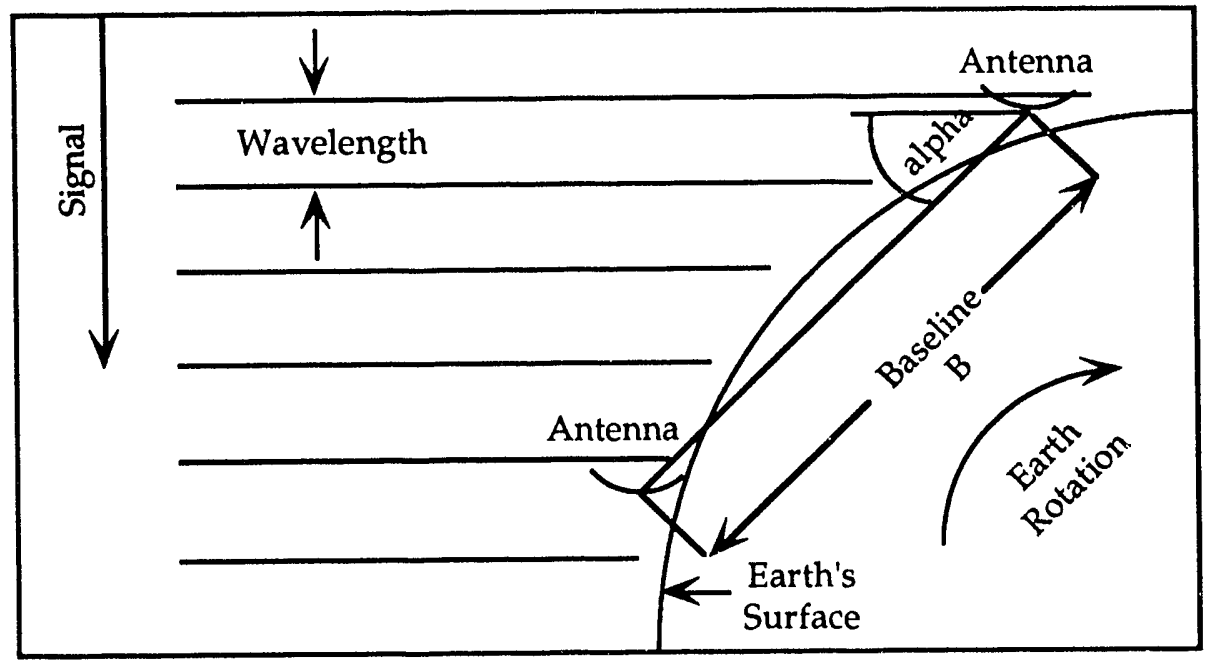

Figure VII.1

VLBI System

When the antennas are far apart, it is impossible to compare their received signals directly or to transmit them to the same location by radio because the phase information, which is the crucial element, would be badly distorted. Instead, the signals are heterodyned at both antennas down to a low frequency using precision local oscillators, usually hydrogen masers. The low-frequency signals, which still have the essential phase information, are then digitized and tape recorded along with timing signals. The tapes are then transported to a location where the phase differences are studied using correlation techniques.

Since the phase of the signal coming out of the receiver at each antenna depends equally on the signal phase and the local oscillator phase, the local oscillator must have extremely good phase stability. Therefore, the phase uncertainty due to noise and random phase drift in the local oscillator at the signal frequency must be less than about a radian over the observation time-which may be several hours. If the phase error gets larger than this, the correlation in the signals disappears and the signal-to-noise ratio suffers. Hydrogen masers are currently the only devices capable of giving this performance. 
The signals being observed are always small, therefore, large antennas and very low-noise, usually cooled, receiving electronics, are required. The digitizer and recorders must also be high quality and wide band. The systems are large and expensive, particularly if a satellite is being considered for operation as one of the stations.

\section{Discussion of Soviet Work}

VLBI not only is valuable for astrophysical investigations, it can also be turned around and used for terrestrial measurements, including the study of motion of tectonic plates (continental drift). Exact knowledge of position is, of course, extremely important to navigation. VLBI systems are used to precisely determine the position of the Earth in an inertial frame represented by distant astronomical radio sources. Regular measurements of the Earth's polar motion and rotation are made by the US Naval Observatory using VLBI data. The Soviet Union has only recently begun to divulge the exact positions of its radio telescopes.

There are radio telescope stations equipped for VLBI located at Simeiz on the Crimean peninsula near Yalta (Matveyenko et al., 1986). These are 22-meter diameter, fully steerable dishes operated at frequencies up to $22 \mathrm{GHz}$. Another antenna is located at Pushchino, about $100 \mathrm{~km}$ south of Moscow. It also operates at $22 \mathrm{GHz}$, but so far has not been used much for VLBI operations. This site also includes a cross-shaped array of dishes operable at $1,025 \mathrm{MHz}$, similar to the array near Bologna, Italy, along with a phased-array of $20-\mathrm{MHz}$ and $70-\mathrm{MHz}$ antennas. Another large antenna is located near Ulan-Ude in the Transbaikal.

There is a 70-meter deep-space tracking station in the Crimea at Yevpatoriya, which was a military station that is still used for space tracking. It is capable of VLBI operation, but is normally not used in international VLBI observing programs. A similar 70-meter deep-space tracking station is located in the Soviet Far East at Ussuriysk, just north of Vladivostok, however, its coordinates have not yet been released.

The Soviet Union is building a third deep-space tracking station in Suffa, near Tashkent. Soviet scientists expect to make it operable at millimeter wave- 
lengths; their present goal is $3 \mathrm{~mm}$. This station is planned to be completed in 1993. Near this site is another 20-meter tracking station.

An active development program is underway for a spaceborne VLBI radiotelescope in the Soviet Radioastron program. During the summer of 1988, when Dr. R. Vessot visited Professor V. Braginskiy at Moscow State University, Dr. N. Kardaschev asked Dr. Vessot to meet with him and other space scientists at the Space Research Institute of the Soviet Academy of Sciences. During this meeting, Dr. Vessot was asked if the Smithsonian Astrophysical Observatory could supply a spaceborne hydrogen maser for the Radioastron program. It was obvious to all that this question could only be answered by NASA and those in the United States in control of the transfer of US technology. At the request of NASA, a summary of the risks and benefits to the United States was developed by R. Vessot and R. Decher of the NASA Marshall Space Flight Center, and it was submitted in early 1989. Thus far, no positive action has been taken by NASA on this question, although NASA will collaborate on the tracking and data acquisition aspects of the Soviet Radioastron program.

Since that time, a new initiative has been taken by the European Space Agency, called the international VLBI satellite. The list of cooperating investigators from all nations involved in this work totals roughly 70 radioastronomers. The international VLBI satellite is a second generation of the Radioastron concept and it will require some form of highly stable spaceworthy oscillator. As yet, there appears to be no official support from NASA for this program, although there are a number of NASA astrophysicists and astronomers involved in the European Space Agency proposal.

The possible division of effort could be as follows:

Vehicle and launch facilities

Satellite

Microwave receivers for the satellite

Tracking stations and Earth VLBI terminal Spaceborne clocks
Soviet Union

European Space Agency

Australia

United States, Australia, Japan

Soviet Union or United States 
The present Radioastron program has received support for spaceborne receivers from the following, couniries:

Finland

Germany

The Netherlands

Australia

India
$23 \mathrm{GHz}$

6-cm band systems

6-cm band systems

$18-\mathrm{cm}$ band systems

$327 \mathrm{MHz}$.

The United States will provide tracking support with the NASA deep-space network, but as of this writing, nothing had been formally concluded.

As mentioned earlier, the VLBI technique requires technology for very highspeed recording and data handling. The United States and Japan clearly have a significant lead in this type of VLBI equipment in the MARK-III terminal. Whether and/or how this technology will be available in the Soviet Union remains to be seen.

Strong points of the Soviet VLBI work include their high-quality scientists (who know and understand what they are doing) and their very good Earthbased hydrogen masers. In addition, these scientists seem to receive strong national support for their efforts. Weak points of the Soviet VLBI work include their inferior recorders and electronics for data handling. Little is known about the quality and ruggedness of Soviet antennas.

\section{Projections for the Future}

Soviet scientists will continue to push for the Radioastron project. They now have competition from the European Space Agency and probably would not like to lose their lead. They have improved in electronics in a number of areas and it seems likely that their recording and data handling capability will also improve. 


\section{QUANTUM MEASUREMENTS}

\section{Summary}

The quantum theory of measurement shows that there are fundamental limits to the stability of oscillators. These are due in part to the fundamental quantum nature of the electromagnetic field and also in the way the measurements are made. While we are still far from reaching some of these limits, it is nevertheless important to know them and use them as guidelines for progress. The Soviet Union is very active in this field and their top scientists are among the world leaders. There has been some very good collaboration between US and Soviet scientists in this area. The Soviet Union appears to be on a par with the West in theoretical aspects, but is lagging in experimental work.

\section{Introduction}

The uncertainty principle is at the heart of all quantum mechanical measurements. Simply stated, the product of uncertainties in the simultaneous measurements of certain pairs of physical variables must be equal to or greater than a certain minimum value. Examples of some of these pairs of variables are $x$ and $p_{x}$ (the $x$ component of posi' ${ }^{\prime}$ on of a particle and its $x$ component of momentum), $J_{z}$ and $\theta_{z}$ (the component of angular momentum of a system about the $\mathrm{z}$ axis and its angle of rotation about the $\mathrm{z}$ axis), the energy $\mathrm{E}$ of a system, and the time $t$ at which it is measured. Such variables are members of the class called "canonically conjugate" in the sense of classical mechanics. The minimum uncertainty product for these variables is $h / 4 \pi$, where $h$ is Planck's constant, $6.626 \times 10^{-34}$ joule seconds. Then, for example $(\Delta x)(\Delta p) \geq h / 4 \pi$, where $\Delta x$ and $\Delta p$ are the RMS uncertainties in $x$ and $p$, respectively. Consequently, the more precisely the $x$ component of the position of a particle is measured, the less precisely the $x$ component of the momentum can be measured immediately afterwards (zero time delay) and vice versa.

By using the uncertainty relation for energy and time, one can arrive at $(\Delta n)(\Delta \phi) \geq 1 / 2$, the uncertainty relation for photon number and phase angle for an electromagnetic mode. This means that if one exactly measures the energy 
(number of photons times the energy per photon) in a mode, then the phase is completely unknown.

Generally, uncertainty relations are caused by physical variables that do not commute with each in the quantum mechanical sense. This, of course, includes the cases mentioned above. There are many other considerations, but it is beyond the scope of this introduction to go much further into detail. Some considerations of direct importance to oscillators are discussed below.

At temperatures such that $\mathrm{k}_{\mathrm{B}} \mathrm{T}>\mathrm{hv} / 2$, a self-excited oscillator has frequency fluctuations governed by thermal noise, provided the resonator frequency is constant. Here, $k_{B}$ is Boltzman's constant, $T$ is the absolute temperature, $h$ is Planck's constant, and $v$ is the oscillator frequency. The quantity $h v / 2$ is the zero point or minimum energy the oscillator can have and is a manifestation of the uncertainty principle. The fractional frequency fluctuations for this thermal case are then given by the well-known formula

$$
\sigma_{\mathrm{y} \text { th }}(\tau)=\frac{1}{\mathrm{Q}} \sqrt{\frac{\mathrm{k}_{\mathrm{B}} \mathrm{T}}{2 \mathrm{P} \tau}}
$$

where $Q$ is the loaded $Q$ of the resonator, $P$ is the power delivered to the loaded resonator, and $\tau$ is the averaging time.

When $k_{B} T<h v / 2$, then the frequency fluctuations are at the fundamental quantum limit, the Townes-Schawlow limit, given by

$$
\sigma_{y q u}(\tau)=\frac{1}{Q} \sqrt{\frac{h v}{4 P \tau}} .
$$

This is simply the thermal noise formula with $k_{B} T$ replaced by the zero point energy, $h v / 2$, where it has again been assumed that the resonator frequency is constant. This limit is fundamental and it is imposed by the uncertainty principle of quantum mechanics. 
Another quantum source of frequency fluctuations is that due to variations in the size of the resonator caused by the quantum fluctuations in radiation pressure acting on the walls. These fluctuations are proportional to $\mathrm{P}^{1 / 2}$ and, in combination with the Townes-Schawlow limit which is proportional to $\mathrm{P}-1 / 2$, give an optimum power level for the minimum total frequency fluctuations. For the best cryogenic oscillators with $Q \approx 10^{11}$ at $10 \mathrm{GHz}$ and achievable material properties, the minimum fluctuations are about $2 \times 10^{-20} / \tau^{1 / 2}$. This is orders of magnituaie better than that which has been achieved to date, but there is much room for improvement.

One can show (Braginskiy and Vorontsov, 1975) that if the coordinate $x$ of a classical mechanical resonator (not self excited) that is very weakly coupled to its surroundings (very high $Q$ ) is measured essentially continuously with a measurement bandwidth that is wide compared to the oscillator frequency, then the fluctuations in the measurement are

$$
\sigma_{x \text { th }}(\tau)=\sqrt{\frac{k_{B} T \tau}{2 \pi v m Q}} .
$$

If the oscillator is completely isolated (infinite $Q$ ), then it is a quantum oscillator and the fluctuations are

$$
\sigma_{x q u}=\sqrt{\frac{h}{8 \pi^{2} v m}}
$$

Combining these two results, we see that if $k_{B} T \tau / Q<h / 4 \pi$, then the oscillator behaves as a quantum system. In other words, if the product of the thermal noise power in the resonators bandwidth, $k_{B} T v / Q$, and the averaging time, $\tau$, is less than the zero point energy, $h v / 2$, the system behaves as a quantum system. This can occur for systems now achievable such as a sapphire mechanical resonator at $2^{\circ} \mathrm{K}$ with $\mathrm{Q}=1 \times 10^{9}$ resonant at $10^{3} \mathrm{~Hz}$ for measuring times shorter than about $1 \times 10^{-3}$ seconds. This is a good example of a macroscopic, but very simple, quantum mechanical system. 
The same analysis can be applied to find the voltage fluctuations in an electromagnetic resonator consisting of an inductance and capacitance (not self excited) by replacing the mass, $m$, in the above formulae with $C /(2 \pi v)^{2}$, where $C$ is the resonator capacitance, and the resonant frequency is $v=1 / 2 \pi(\mathrm{LC})^{1 / 2}$, with $\mathrm{L}$ being the resonator inductance.

Another area of theoretical and possible practical importance is called quantum nondemolition (QND) measurement. If one makes any kind of a measurement $r$ on a system and gets a result $R$ and then immediately (zero time later) makes the same measurement, he gets exactly the same result, $R$. This is an essential part of measurement theory and the interpretation of quantum mechanics. The act of making the measurement puts the system in an eigenstate of the measurement operator so that repeated immediate measurements always give the same result. If the system is allowed to evolve freely in time between the measurements, successive measurements may or may not give the same results. If they do, they are QND measurements. Thus, a QND measurement is ideal for determining whether the system changed states due to an influence other than the measurement, and is the reason for much of the interest.

A simple example of a QND measurement, in principle, is the momentum of a free particle in one dimension. Once a measurement has been made with result $p$, the system is in an eigenstate of the momentum operator with eigenvalue $p$. Momentum eigenstates are also eigenstates for a free particle, so the results will always give $p$ independent of the time between measurements. Any operator that commutes with a time-independent Hamiltonian such as that for a free particle will behave in this manner and will therefore be a QND measurement operator.

The measurement of position of a free particle is not a QND measurement. Making the first measurement of position puts the particle in a position eigenstate, but now the momentum is spread out and is uncertain because the particle is not in a momentum eigenstate. Consequently, since the position of the particle at later times depends not only on its initial position but also on its momentum, there will be a spread in later position measurements due to the spread in momentum induced by the previous position measurement. This effect is called back action. It occurs with any operator that does not commute with the Hamil- 
tonian or, as can be shown, when the operator in the Heisenberg representation does not commute with itself at different times. The position operator for a free particle is such an operator.

Quantum nondemolition measurements potentially are important because they allow measurements to be made without the above-mentioned back action. Their application to gravity wave detectors, which use very large bars as mechanical harmonic oscillators, is currently a subject of considerable interest.

While in many cases it is possible to find a measurement that is QND in principle, it may be difficult if not impossible to implement. For example, momentum measurements on a free particle are QND, but to date, it is not known how to make sufficientiy precise momentum measurements to be useful.

An interesting situation arises with the harmonic oscillator. Here, it can be shown that the commutator of the position operator with itself at different times is a periodic function of the time difference with the same period as the oscillator. The same is true for the momentum operator. Under these conditions, the commutator vanishes each time that the time difference is a multiple of the oscillator half period. Therefore, if the position measurement is always performed at these times, it is QND. This is called a stroboscopic measurement. Note, however, that the uncertainty product must satisfy the uncertainty relation at all times. There are other types of QND measurements for harmonic oscillators, such as measuring the real or imaginary part of the complex amplitude, but these are beyond the scope of this introduction.

Another area of interest is the concept known as squeezed quantum states. These are harmonic oscillator states whose uncertainties oscillate in time in contrast to the constant uncertainties of the well-known minimum uncertainty coherent state. Figure VII.2 presents phase plane plots of three situations. Figure VII.2a represents a coherent oscillator state which has minimum uncertainties in $x$ and $p$, coordinate and momentum, respectively. The uncertainties are represented by the circle and, as it moves about the origin in the direction indicated, these uncertainties do not change. Figure VII. $2 b$ represents a squeezed state whose uncertainties in $x$ and $p$ are minimum when $x$ or $p$ are zero. This is a phase-squeezed state. Note that the minimum uncertainty in $x$ or $p$ is reached 
twice in each oscillator cycle. Figure VII.2c represents the state with maximum uncertainties at zero $x$ or $p$. This is an amplitude-squeezed state. The previously mentioned stroboscopic QND measurement puts the oscillator in a squeezed state. Squeezed states can only be achieved with nonlinear or time-varying measurement operators. They are of interest because, in principle, they allow measurements to be made with lower noise than can be made with coherent states.

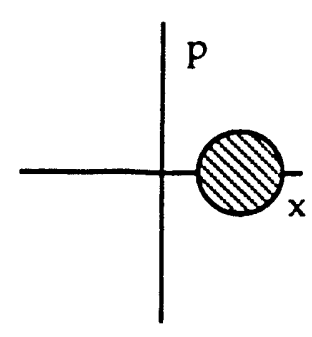

(a)

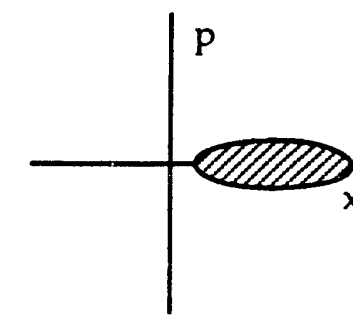

(b)

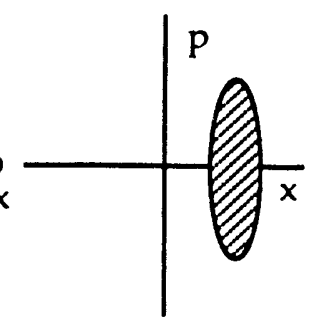

(c)

(a) Coherent state with constant uncertainties as the phasor rotates. (b) Squeezed state with minimum uncertainty in $x$ or $p$ at $x$ or $p=0$. (c) Squeezed state with maximum uncertainty in $x$ or $p$ at $p$ or $x=0$.

Figure VII.2

Harmonic Oscillator States

The field of quantum measurement is still a subject of considerable activity and debate among top theorists in the world. It is important for understanding the ultimate performance of frequency standards.

\section{Discussion of Soviet Work}

In 1980, a joint paper by two top Soviet scientists and a top US scientist, Kip Thorne, reviewed QND measurements (Braginskiy et al., 1980). These authors continue to collaborate on a great deal of excellent fundamental work. Their paper reviewed much of the status up to that time and showed that there were strong contributions from both the West and the Soviet Union. The idea of 
using QND for gravitational wave detectors is credited to Braginskiy, but the fundamental idea of QND measurements goes back to Von Neuman, more than 35 years ago. The fundamental effect of quantum fluctuations in the radiation pressure on cavity frequency and, consequently, frequency stability of oscillators was a Soviet contribution. It is clear that the Soviet Union was on a par with the West in theory at that time.

Further work on oscillator stability and fundamental measurement limitations is covered in excellent book by Braginskiy et al. (1985), where the quantum behavior of very high $Q$ resonators with regard to measurement of the generalized position coordinate (position in the case of a mechanical resonator and voltage across a capacitor in an electromagnetic resonator) is discussed. QND measurements are also covered, in particular, stroboscopic and quadrature-amplitude measurements. Contributions from the East and West here are clearly stated. Again, the Soviet work is on a par with that of the rest of the world.

Recent frequency stability performance for various frequency standards and fundamental quantum limitations are discussed in another review paper by Braginskiy (1988). However, most of the experimental results reported are from the West. This paper also reported on expectations for cryogenic hydrogen masers. The standard quantum limit is still many orders of magnitude better than the best experimental results. Stroboscopic types of measurement were also discussed, as well as other squeezed-state techniques.

A technique for the QND measurement of the energy or frequency of a group of photons in a waveguide was discussed theoretically by Braginskiy and Khalili (1988). It was shown that this leads to special quantum states for the group which were termed frequency anticorrelated states. Such states can be used in the Doppler measurement of momentum of a free-mass gravitational antenna, and they give an asymptotically QND energy or momentum measurement as the averaging time becomes long.

There is some Soviet work on squeezed states, most of it theoretical. Judging from the number of papers cited, there is considerably less work going on there than in the West. 
A study was conducted on the generation of optical squeezed states in parametric amplification with a noisy pump wave or four wave mixing with noisy reference wave (Oraevskiy, 1987). It is shown that both amplitude and phase fluctuations reduce the squeezing that can be achieved; however, if only ampiitude fluctuations are present and they are small enough, then squeezing is not limited. Phase fluctuations always affect the squeezing. This work was motivated by the fairly small squeezing reported in experiments.

Degradation of the squeezing of states in an optical field due to diffraction was also treated (Akhmanov et al., 1988). Experiments, mostly in the United States, had demonstrated squeezing using phase-sensitive parametric amplification. The reported noise reduction achieved in homodyne detection was not very large, and considerably better performance could be expected theoretically. An explanation was made in terms of loss of phase match in the propagating beams due to the diffraction from a finite-size optical beam compared to the ideal plane wave behavior.

\section{Projections for the Future}

Soviet work will continue in this area, probably increasing as time passes and experimental results get closer to the fundamental limits. The good association and collaboration of Braginskiy with Western scientists undoubtedly will continue. Squeezed states could play an important role in communications because of their noise reduction properties, and this is always valuable in improving performance and reducing cost. Braginskiy and Khalili, along with the collaboration of the prominent US scientist, Kip Thorne, are preparing a book entitled Quantum Measurements which will be published in the United States in 1991.

\section{E. KEY SOVIET RESEARCH PERSONNEL AND FACILITIES}

A list of key Soviet researchers involved in relativity and gravitation, astrophysics and very long baseline interferometry, and quantum mechanics research is provided in Table VII.i. 
Table VII.1

KEY SOVIET RESEARCH PERSONNEL AND FACILITIES-

RELATIVITY AND GRAVITATION, ASTROPHYSICS AND VERY LONG

BASELINE INTERFEROMETRY, AND QUANTUM MECHANICS

\section{RELATIVITY AND GRAVITATION}

Moscow State University im. V. M. Lomonosov, Moscow

A. A. Logunov

Yu. M. Loskutov

Academician and Rector of University; theoretical physics Theoretical physics

Physics Institute im. P. N. Lebedev, AS USSR, Moscow

N. G. Basov

L. B. Borisova

V. N. Mel'nikov
Nobel Prize winner; masers and optical physics

\section{Unknown Affiliation}

Theoretical physics

Theoretical physics

\section{ASTROPHYSICS AND VERY LONG BASELINE INTERFEROMETRY}

Space Research Institute, AS USSR, Moscow
N. S. Kardashev
L. I. Matveyenko
L. Gurvitz
V. Slysh
M. Popov
L. R. Kogan
V. Andreyanov

\author{
Deputy Director \\ Director of Crimean Operations \\ Project Scientist for Radioastron \\ Head of Radioastronomy Division \\ Head of Science \\ VLBI on celestial $\mathrm{H}_{2} \mathrm{O}$ masers \\ Head of tracking electronics
}

Other Facilities

Radiophysics and Electronics Institute, AS USSR, Yerevan Main Astroiomicai Observatory, AS UkSSR, Goloseyev (Kiev) 
Table VII.1

KEY SOVIET RESEARCH PERSONNEL AND FACILITIES-

RELATIVITY AND GRAVITATION, ASTROPHYSICS AND VERY LONG

BASELINE INTERFEROMETRY, AND QUANTUM MECHANICS (cont'd.)

\section{QUANTUM MECHANICS}

Mossow State University im. V. M. Lomonosov, Moscow

V. B. Braginskiy

Professor of Physics, Chair of Radiophysics and Electronics Division (gravity-wave detectors and very high-quality oscillators)

F. Ya. Khalili

Colleague of Braginskiy, a very strong mathematical physicist

V. P. Mitrofanov

Senior Scientist

V. I. Panov

Senior Scientist

Yu. I. Vorontsov

Associate Professor of Physics 


\section{CHAPTER VII: RELEVANT SCIENCE APPLICATIONS REFERENCES}

Akhmanov, S. A., A. V. Belinskiy (Belinskii), and A. S. Chirkin, "Squeezed States in the Case of
Parametric Amplification in Diffracted Light Beams," Sov. J. Quantum Electron, 18, 5(1988), 560561.

Basov, N. G., M. I. Borisenko, S. P. Vlasov, N. Ye. Dubonosov, and G. M. Ivanov, "Operational Test of a Molecular Generator on an Artificial Earth Satellite," Cosmic Res., 5, 4(1967), 608-616.

Borisova, L. B., K. A. Bronikov, and V. N. Mel'nikov, "Taking into Account Gravitational and Relativistic Effects in Maintaining a Unified Time Scale on Earth and Surrounding Space," Meas. Tech., 31, 5(1988), 450-455.

Borisova, L. B., and V. N. Mel'nikov, "Relativistic Corrections to Readings from a Portable Clock," Meas. Tech., 31, 4(1988), 323-327.

Braginskiy (Braginskii), V. B., "Resolution in Macroscopic Measurements: Progress and Prospects," Sov. Phys.-Usp., 31, 9(1988), 836-849.

Braginskiy (Braginskii), V. B., and F. Ya. Khalili, "Frequency-Anticorrelated Quantum States," Sov. Phys.JETP, 67, 1(1988), 84-88.

Braginskiy (Braginskii), V. B., V. P. Mitrofanov, and V. I. Panov, Systems with Small Dissipation, Chicago: University of Chicago Press, 1985.

Braginskiy (Braginskii), V. B., and Yu. I. Vorontsov, "Quantum Mechanical Limitations in Macroscopic Experiments and Modern Experimental Techniques," Sov. Phys.-Usp., 17, 5(1975), 644-650.

Braginskiy (Braginskii), V. B., Yu. I. Vorontsov, and K. S. Thorne, "Quantum Nondemolition Measurements," Science, 209, 1(1980), 547-557.

Logunov, A. A., and Yu. M. Loskutov, "Delay of Radio Signals in the Relativistic Theory of Gravitation," Sov. Phys.-Dokl., 30, 11(1985), 971-972.

Logunov, A. A., and Yu. M. Loskutov, "Contradictory Character of General Relativity. The Relativistic Theory of Gravitation," Theor. Math. Phys., 67, 2(1986), 425-433.

Matveyenko (Matveenko), L. I., R. Z. Sagdeyev (Sagdeev), V. M. Balebanov, V. I. Shevchenko, V. I. Kostenko, V. A. Grishmanovskiy (Grishmanovskii), V. Ye. Velikhov, S. P. Ignatov, B. Z. Kanevskiy (Kanevskii), L. R. Kogan, G. D. Kopelyanskiy (Kopelianskii), A. N. Kozlov, A. P. Molodyanu (Molodianu), E. P. Molotov, A. Kh. Papatsenko, A. M. Romanov, A. V. Shevchenko, I. A. Strukov, V. V. Timofeyev (Timofeev), A. B. Severnyy (Severnyi), I. G. Moyseyev (Moiseev), R. L. Sorochenko, A. P. Tsivilev, R. M. Martirosyan (Martirosian), A. M. Aslanyan (Alsanian), A. G. Gulyan (Gulian), Ya. S. Yatskiv (Iatskiv), and M. V. Golovnya (Golovnia), "The Soviet 18-cm Wavelength VLBI Network," Sov. Astron. Lett., 12, 1(1986), 25-28.

Orayevskiy (Oraevskii), A. N., "Generation of Squeezed States by a Fluctuating Pump Wave," Sov. J. Quantum Electron., 17, 8(1987), 967-968. 
(blank)

VII-26 


\section{CHAPTER VIII \\ AVAILABLE SUPPORTING TECHNOLOGY}

\section{A. SUMMARY}

In addition to the underlying physics, each type of atomic standard relies on additional electronic circuitry to realize the inherent stability of the atomic resonance in a physical output. These circuits include oscillators, frequency multipliers, synthesizers, laser sources, and low-noise electronics. The published Soviet literature on atomic clock research contains little detailed information on the type of technologies used for these functions. A review of these specific technical areas reveals that the Soviet Union has capabilities at least comparable to those of the United States in several key areas.

The quartz crystal oscillators available for use in clocks include not only the classic thermally controlled, or "ovenized," oscillator that has been widely used in the West, but also low-noise versions and digital-thermal compensation oscillators that can provide performance comparable to ovenized oscillators at a small fraction of the power, weight, and size. Low-noise oscillators are needed to allow the interrogation of very narrow linewidths and to provide output signals of very high spectral purity. The Soviet state of development as of 1985 was significantly below that of the United States and would not have been adequate for use with ion storage clocks. Soviet researchers have developed a digital-thermal compensation scheme that is applicable to small, low-power clocks for portable air or space operations. The last reported work on such a scheme is in 1986; it indicates that the Soviets understand the concept even though their implementation at that time was not as polished as current Western devices.

The Soviet Union has demonstrated a capability to make numerically controlled synthesizers that use digital-to-analog converters to create small, precisely controlled frequency steps. These devices can be used in atomic standards to allow precise adjustment of the clock's output frequency without disturbing the internal atomic resonance. Clocks controlled in this manner are especially useful in creating clock ensembles where the physical output of one clock is controlled by a computer to slave it to an averaged output of a group of clocks. This 
technique is also useful in controlling the frequency of a clock in a remote location to broadcast time reference signals, such as in the GLONASS system.

Frequency multipliers and low-noise electronics are also key parts of clock servo-control loops. There are few references to unique devices in the published Soviet literature. However, by observing the achieved performance of Soviet clocks, it is possible to make the assumption that the Soviet Union has capabilities comparable to those of the United States.

Soviet scientists have used laser light sources for laser frequency standards and for stable sources of light for optical state selection in atomic frequency standards.

\section{B. QUARTZ CRYSTAL OSCILLATORS}

The quartz oscillator is the basis of all modern timekeeping. Since the development of practical electronic circuits early in this century, electrical timekeeping has rapidly supplanted mechanical and astronomical methods. The crystal element works as a narrowband transducer converting electrical energy to mechanical vibrations in its lattice structure and then back again to an electrical signal. $Q$ factors for the highest quality crystals are on the order of $10^{7}$. Placed in a feedback loop in an oscillator circuit, these devices are capable of producing radio frequency output signals with a frequency stability of a few parts in 10-14. ${ }^{1}$ The Soviet Union is also known to have naturally occurring raw quartz of exceptionally high quality.

The problems with using crystal oscillators for high-precision applications arise from their inherent instabilities due to temperature, vibration, and longterm frequency drift. Improvements made to the basic technology include temperature control of the crystal with an oven, temperature compensation by passive and active means, rotated cuts of the crystal blank to optimize particular parameters, and careful processing and mounting of the resonator. 
One of the key parametors in the manufacture of quartz crystal resonators is the angle at which the resonator is cut with respect to the crystallographic axes of the quartz material. The properties of quartz vary greatly depending on the crystallographic direction in which a property is measured. For example, the coefficient of thermal expansion and the temperature coefficients of the elastic properties of the materials can change dramatically. Today's common crystal cuts are chosen in an effort to optimize performance. Figure VIII.1 illustrates several common crystallographic orientations. Note that some cuts are rotated only in the theta direction while others are rotated in both the theta and phi angles. The AT cut, until recently, has been favored for high-precision quartz clocks. Recent improvements in measurement of the crystallographic axes have made it possible to produce doubly rotated cuts, such as the SC cut. Resonators using the SC cut have proven to be significantly better than the older, AT-cut units.

The published literature reveals very little about the Soviet capability in lownoise oscillators. Vishina and Yelkin (1988) claim a stability of about $1 \times 10^{-12}$ at 1 second. This can be contrasted to the $5 \times 10^{-14}$ stability reported by Bloch et al. ${ }^{2}$ and the $3 \times 10^{-13}$ stability achieved by Brandenberger et al. ${ }^{3}$ The limiting factor to stability is sensitivity to the level of the amplitude of the oscillator. Vishina and Yelkin state that an .01 percent amplitude regulation is required to achieve a frequency stability of $1 \times 10^{-12}$. The Soviet Union continues to use the AT-cut crystal which is no longer widely used for high precision in the West. The SCcut crystal has better noise, vibration, and temperature performance. In 1985, R. L. Filler showed that the SC cut is roughly five times less sensitive to drive level than the AT cut for a $5.115-\mathrm{MHz}$ third overtone crystal. ${ }^{4}$ The Soviet Union is aware of the SC cut, but it is apparently not widely used there (Bagayev et al., 1986).

2 M. B. Bloch, M. Meirs, and J. C. Ho, "The Microcomputer-Compensated Crystal Oscillator (MCXO)," 43rd Annual Symp. on Frequency Control, 1989, 16.

3 H. Brandenberger, F. Hadorn, D. Halford, and J. H. Shoaf, "High-Quality Quartz Crystal Oscillators: Frequency Domain and Time Domain Stability," 25th Annual Symp. on Frequency Control, 1971, 226.

4 R. L. Filler, "The Amplitude-Frequency Effect in SC-Cut Resonators," 39th Annual Symp. on Frequency Control, 1985, 311. 

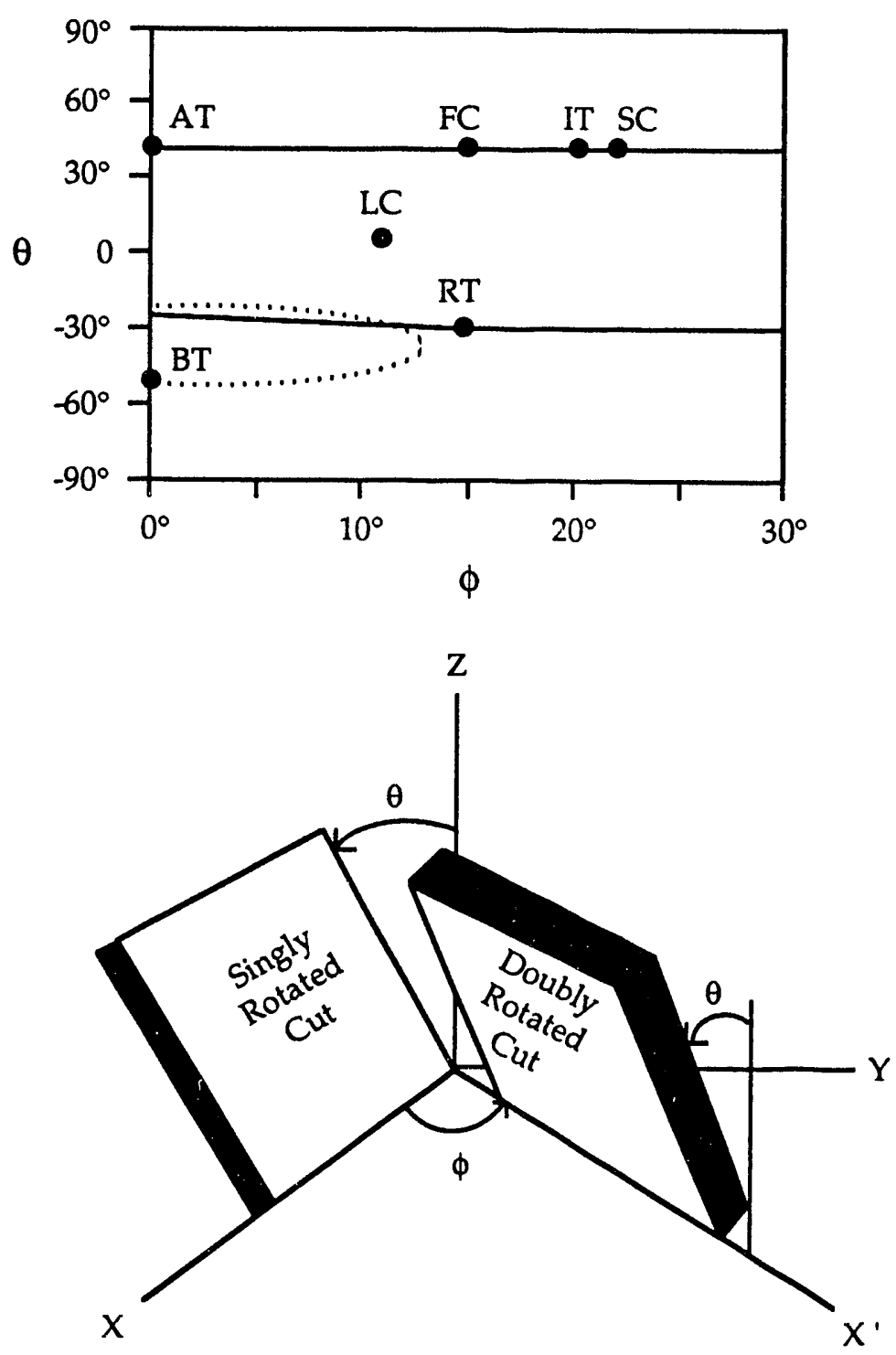

$\mathrm{Y}$ cut $\approx+90 \mathrm{ppm} /{ }^{\circ} \mathrm{C}$ (thickness-shear mode); $\mathrm{X}$ cut $\approx-20 \mathrm{ppm} /{ }^{\circ} \mathrm{C}$ (extensional mode).

Figure VIII.1

Doubly Rotated Quartz Cuts ${ }^{5}$

5 J. Vig, Quartz Crystal Resonators and Oscillators, US Army LABCOM, 1987. 
At a performance level of about $1 \times 10^{-12}$, the Vishina oscillator is adequate for cesium, rubidium, and hydrogen maser standards. In cesium clocks, the inherent noise in the atomic servo loop is greater than that of the oscillator for averaging times of 1 to 10 seconds. Beyond that time, the servo control loop corrects the oscillator. In rubidium standards and hydrogen masers, the very short loop time constant in the servo also compensates for the noise in the oscillator and makes possible the good short-term stabilities below 10,000 seconds for which these clocks are known. Oscillators comparable to the Vishina oscillator are used on most modern Western clocks.

Ion storage clocks require better performance than that which is available from the Vishina quartz oscillator. The atomic resonance for the mercury ion clock is at about $40.5 \mathrm{GHz}$. When the frequency of the flywheel oscillator is multiplied to that frequency to interrogate the atomic line, the oscillator's noise is also multiplied with it (noise increases as the square of the multiplication factor). If the oscillator does not have exceptional short-term stability and spectral purity, the measurement of the resonance will be less precise. For example, the linewidth of the mercury ion transition is less than $1 \mathrm{~Hz}$. Therefore, an oscillator with a short-term stability in the 10-11 range will only allow the narrow resonance line to be barely detected. By comparison, a cesium clock will usually have a linewidth of several hundred hertz at a frequency of $9 \mathrm{GHz}$, and the situation is roughly 10,000 times less critical. In practice, hydrogen masers are used for interrogation sources in the ion storage clocks at the US Naval Observatory. ${ }^{6}$

In the area of environmental sensitivity, there have been two major advancements over the past 10 years. These include the development and use of the SC-cut crystal, and the use of digital frequency compensation of thermal effects using dual-mode excitation of the crystal.

As previously noted, Soviet researchers are aware of the SC cut, but there was no mention of its use in the published literature. This is significant because the SC cut is the most widely used crystal in military and high-precision oscilla-

6 L. S. Cutler, R. P. Giffard, P. J. Wheeler, and G. M. R. Winkler, "Initial Operational Experience with a Mercury Ion Storage Frequency Standard," 41st Annual Symp. on Frequency Control, 1987, 12. 
tors in the West. For temperature-controlled ovenized precision oscillators, the manufacturing processes in SC cuts are critical. There are relatively few Western sources, and the yield of usable crystals is low. Export of technology for manufacturing the doubly rotated cuts is controlled by COCOM. There is no indication in the published literature as to whether or not the Soviet Union has developed a serious capability to manufacture SC-cut crystals.

In addition to the reduction in sensitivity to the crystal drive level, the SC cut is superior to the AT cut in two other critical areas. The first advantage is temperature sensitivity at the normal operating point. Most crystal cuts exhibit a "turnover point" in their frequency-versus-temperature response where the slope of the curve is zero as the direction of the temperature sensitivity changes, as shown in Figure VIII.2. The cut of the crystal is optimized to put this point at a convenient temperature. A typical ovenized oscillator will be set to control the temperature at $75^{\circ} \mathrm{C}$. This allows the oven to always have range for thermal control by adding heat for temperatures up to about $60^{\circ} \mathrm{C}$. A typical AT-cut crystal will have a temperature slope of a few parts in $10^{8}$ per degree on either side of the turnover point. The performance of the SC-cut resonator is more than 10 times better (see Figure VIII.2). ${ }^{7}$ The second major advantage to the SC cut is its reduced sensitivity to vibration. Oscillators using AT-cut crystals usually have a vibration sensitivity on the order of $1 \times 10^{-9}$ per G. Clocks using SC-cut crystals have demonstrated improved performance on the order of $3 \times 10^{-10}$ per G. 8

Temperature and vibration sensitivity are important for atomic standards. Because the servo control loop must keep the frequency of the crystal oscillator locked to that of the atomic resonance, it must be able to follow and correct changes in the crystal's output frequency. By reducing the magnitude of the frequency excursions that the servo has to control, the stress on the loop is reduced. There is also a design trade-off in the servo system between the magnitude of the frequency range to be covered and the gain of the servo. Vibration effects on the output frequency of an atomic clock are nearly the same as they are on its inter-

7 R. Burgoon and R. L. Wilson, "Design Aspects of and Oscillator Using the SC-Cut Crystal," 33rd Annual Symp. on Frequency Control, 1979, 411.

8

B. T. Milliren, D. W. Martin, and D. A. Emmons, "An Acceleration-Compensated Precision Quartz Osciliator," 42ind Annuai Symp. on Frequency Control, 1988, 334. 
nal crystal oscillator. Vibration of the oscillator causes a mechanical perturbation on the crystal itself. Those vibrations are reproduced at the output of the oscillator in the form of phase-noise sidebands separated from the carrier by the frequency of the vibration. That is, an oscillator operating on a platform that is vibrating at a $50-\mathrm{Hz}$ rate will have noise in its output spectrum $50 \mathrm{~Hz}$ above and below the primary output frequency. The magnitude of the noise is proportional to the magnitude of the vibration. Standards operated in a laboratory environment experience little or no vibration. On the other hand, clocks on aircraft, ships, or land vehicles are exposed to strong mechanical vibration. The servo control loops do not operate fast enough to remove these frequency errors.

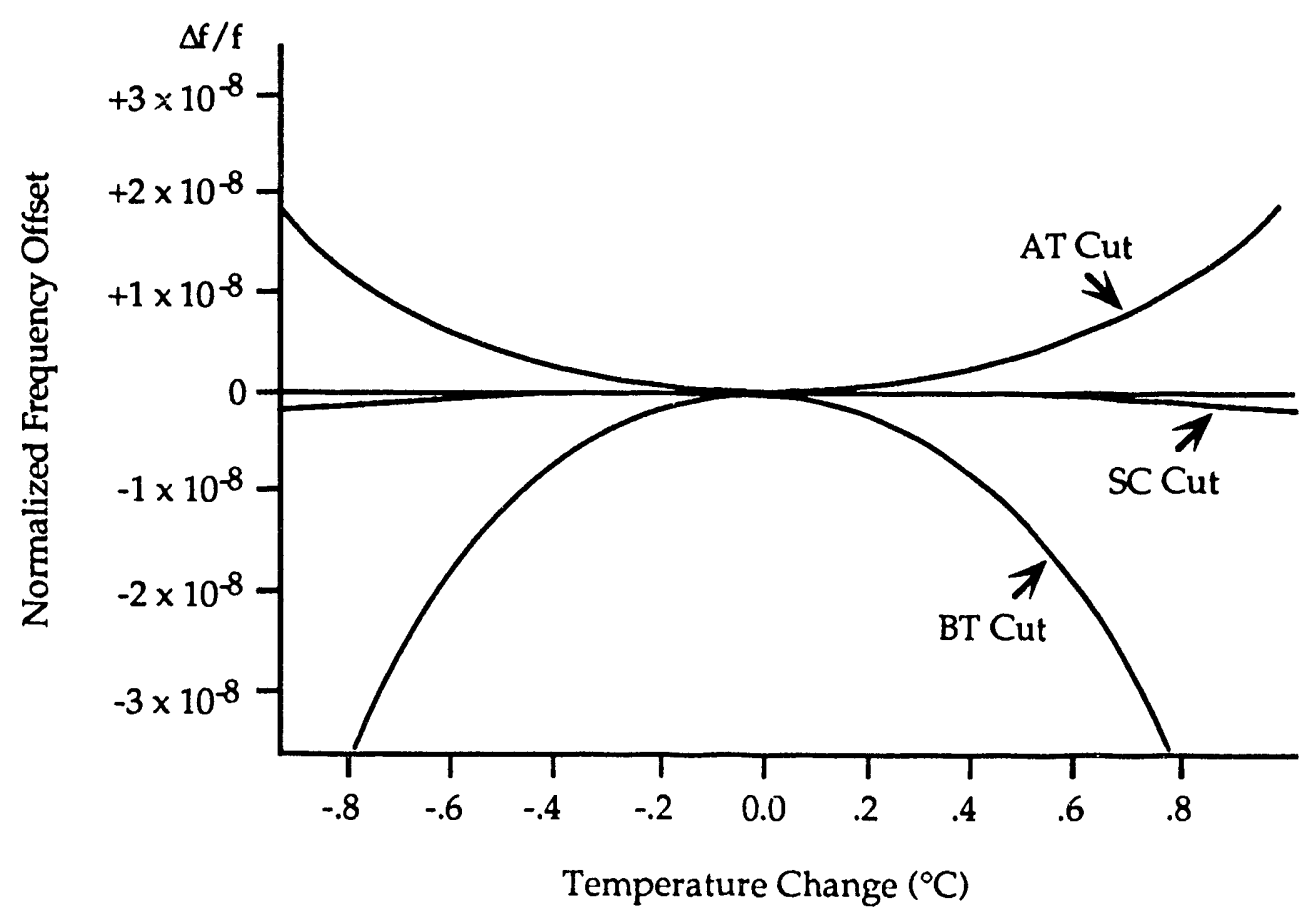

Figure VIII.2

Crystal Temperature Performance Close to the Turnover Temperature

Another development which has some bearing on atomic standards is the use of dual-mode excitation of the crystal to achieve digital-thermal compensation. Until recently, high-performance oscillators used ovenized temperature control to maintain the crystal at its turnover point operating temperature. 
Other types of computer-controlled thermal compensation schemes have been tried, but with several orders of magnitude poorer success. ${ }^{9}$ In 1978, Kusters and Leach laid out the mechanism for simultaneously using two modes of crystal oscillation. ${ }^{10}$ Each mode has a different temperature coefficient. The difference between the two output frequencies is directly related to the crystal temperature, and can be used very accurately to determine the true temperature of the resonator. Prior methods of temperature compensation relied on measuring the temperature at a point near the crystal, but could not measure its temperature directly. These oscillators are referred to as temperature-compensated crystal oscillators; they are subject to hysteresis and similar effects which are introduced into the compensation process. The typical limit for frequency control over the military temperature range $\left(-40\right.$ to $\left.+65^{\circ} \mathrm{C}\right)$ was a few parts in $10^{6}$ for these oscillators. The new approach has yielded results 100 times better. Kusters' device was a laboratory demonstration unit which was not practical for field use due to the size and power of the components needed. Little new work was reported until 1989, when Filler et al. presented a device known as a microcomputer-controlled crystal oscillator (MCXO). The clocks cited by Filler were built by two US companies-Frequency Electronics and General Technical Services. ${ }^{11}$ The reason that little was reported was that the work being conducted by the US Army on this concept was classified from 1982 to 1989 . The clock, which was revealed in 1989, was a major improvement over previous work. It used no more power than a temperature-compensated crystal oscillator, had similar temperature performance to an ovenized oscillator, and showed promise of being inexpensive to produce in quantity (see Figure VIII.3).

9 R. L. Filler, J. A. Messina, and V. J. Rosati, "Frequency-Temperature and Aging Performance of Microcomputer-Compensated Crystal Oscillators," 43rd Annual Symp. on Frequency Control, 1989, 27.

10 J. A. Kusters, M. C. Fischer, and J. G. Leach, "Dual-Mode Operation of Temperature- and Stress-Compensated Crystals," 32nd Annual Symp. on Frequency Control, 1978, 389.

11 M. B. Bloch, M. Meirs, and J. C. Ho, "The Microcomputer-Compensated Crystal Oscillator (MCXO)," 43rd Annual Symp. on Frequency Control, 1989, 16.

A. Benjaminson and S. C. Stallings, "A Microcomputer-Compensated Crystal Oscillator Using a Dual-Mode Resonator," 43rd Annual Symp. on Frequency Control, 1989, 20. 


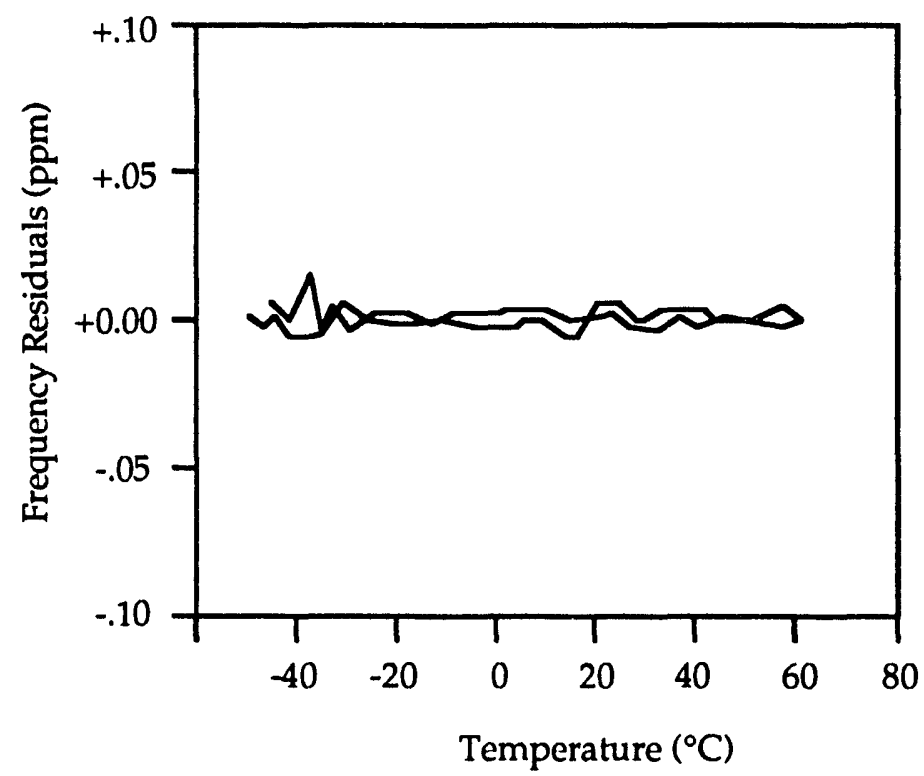

Figure VIII.3

Residuals vs. Temperature for the TFR Dual-Mode Oscillator SN AP7

In 1986, Bagayev et al. reported an oscillator with most of the features of the MCXO. It was a lightweight, low-power, self-contained unit using dual-mode oscillation and digital compensation. The temperature compensation (Figure VIII.4) clearly is within the $\pm 5 \times 10^{-8}$ range achieved by Filler. It is interesting that the shape of the frequency versus the temperature curve is not the nearly random response of Figure VIII.3, but instead is the poorly interpolated result shown by Kusters' work from 1978 (Figure VIII.5). The difference is in the nature of the interpolation. Filler used quadratic interpolation; Bagayev used a linear scheme. Since Kusters also reported using an alternate quadratic method (Figure VIII.6), there is no reason to believe that the Soviet Union has not since adopted this method and can now come much closer to Filler's results. One major shortcoming of Bagayev's work is that the crystal he used was an AT-cut rather than an SC-cut crystal. Again, the apparent Soviet lack of an SC capability hinders the performance of their quartz oscillators. There is an important difference in the MCXO application. Here, the accuracy of the cut is not as critical. The reason is that an MCXO is designed for a crystal with a turnover temperature near ambient, which is a point where the performance is relatively insensitive to the precision of the cut. Table VIII.1 shows a comparison of the reported results. In addi- 
tion to the MCXO application, the Soviet Union is also looking at improving the performance of its ovenized oscillators by using dual-mode operation to provide the temperature error information to control the oven (Fromberg and Yampolskiy, 1986). That scheme has not yet been implemented in the United States.

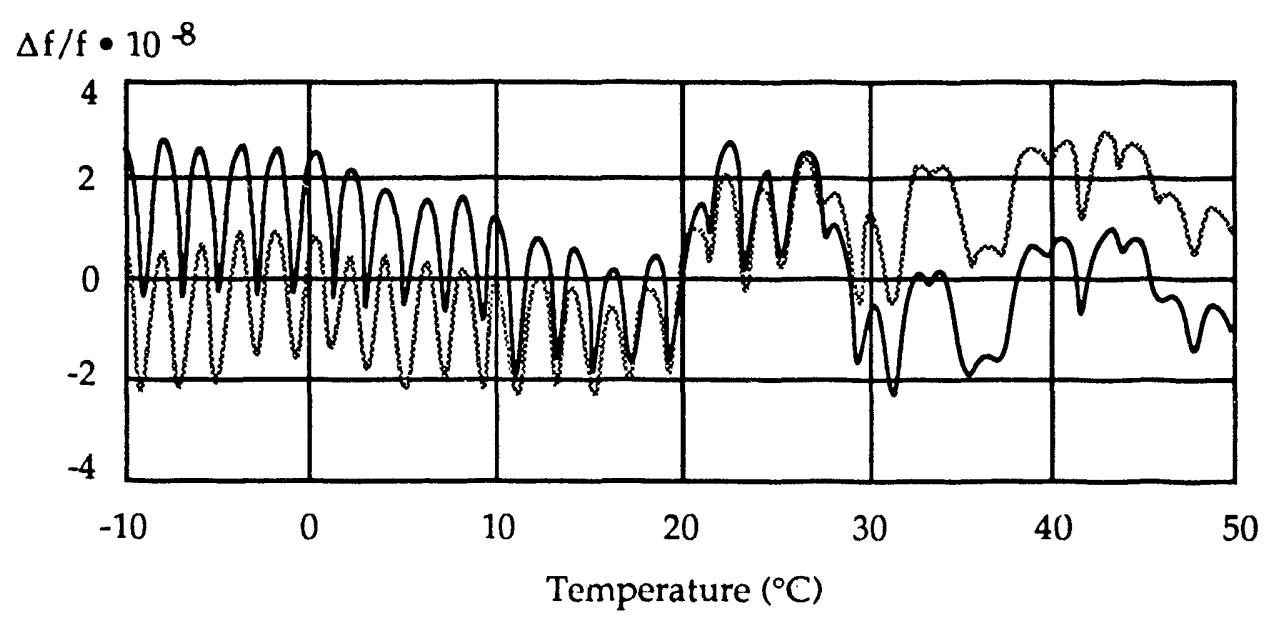

Figure VIII.4

Temperature Compensation for a Soviet Oscillator

(Bagayev et al., 1986)

To date, MCXO oscillators have not been applied to atomic clocks. The driving force in their creation was finding a low-powered replacement for the ovenized oscillator for low-power applications such as field radios. Since the declassification of the MCXO in 1989, only eight units have been delivered in the United States. There is no indication as to how many oscillators the Soviet Union may have made, but it is reasonable to assume that their primary application is the same as that of the United States. The most appropriate use of the $\mathrm{MCXO}$ in atomic standards would be in ruggedized clocks used in field or space applications where small size and low power consumption are particularly important. There are limitations to application of the present MCXO devices in atomic clocks. The phase noise of the MCXO unit, as shown in Figure VIII.7, illustrates the increase in noise around the carrier near the correction rate. That level is still below what is found in some cesium clock oscillators, but it is cer- 
tainly not appropriate for hydrogen masers or even rubidium clocks. Bagayev's oscillator also showed a spurious output at the frequency of the second mode which was stronger than 80 decibels below the primary output. A crystal filter on the primary output would remove the unwanted component, but would add additional sensitivity to temperature and vibration.

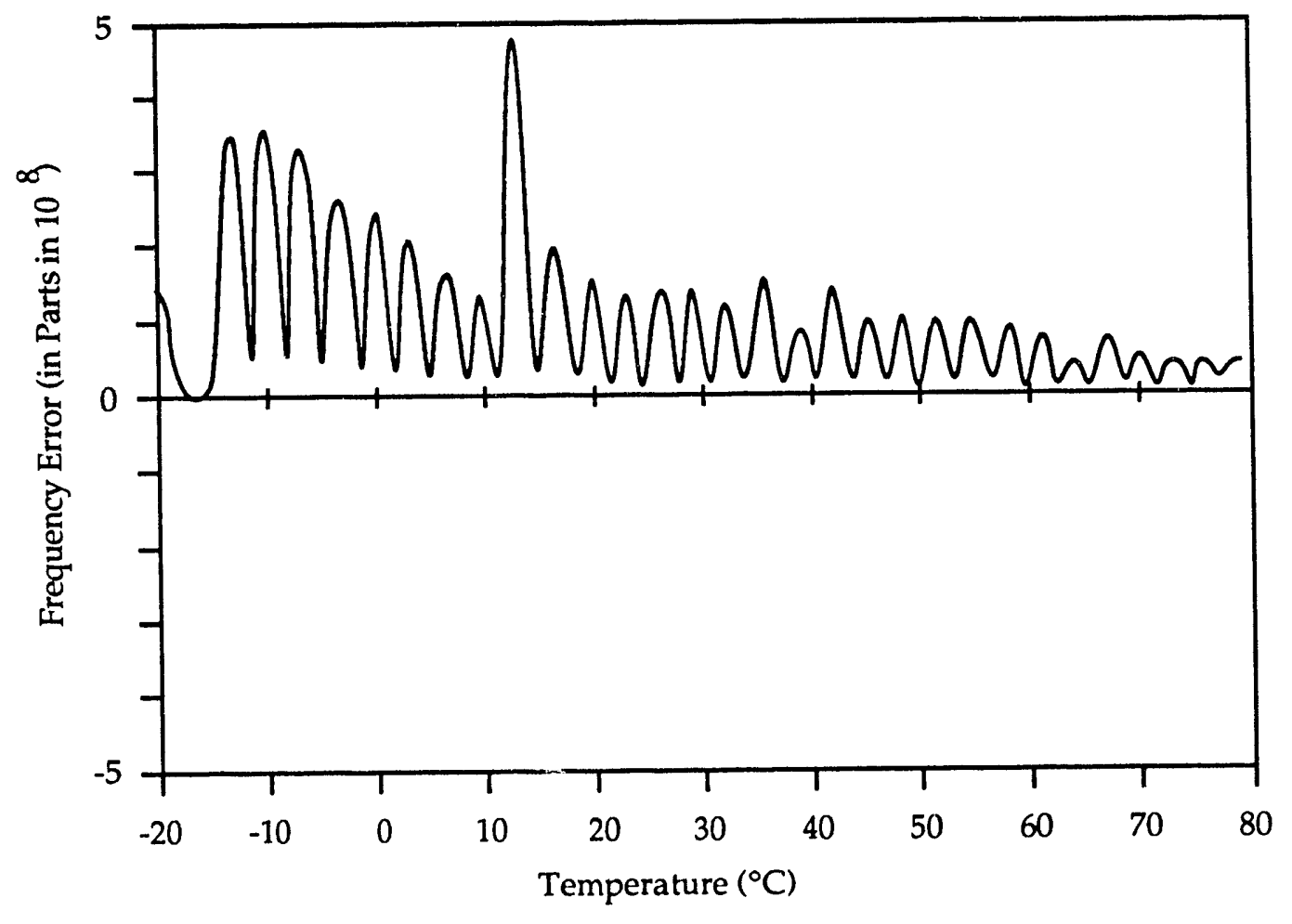

Figure VIII.5

Compensation Error: Linear Interpolation, 30 Points 


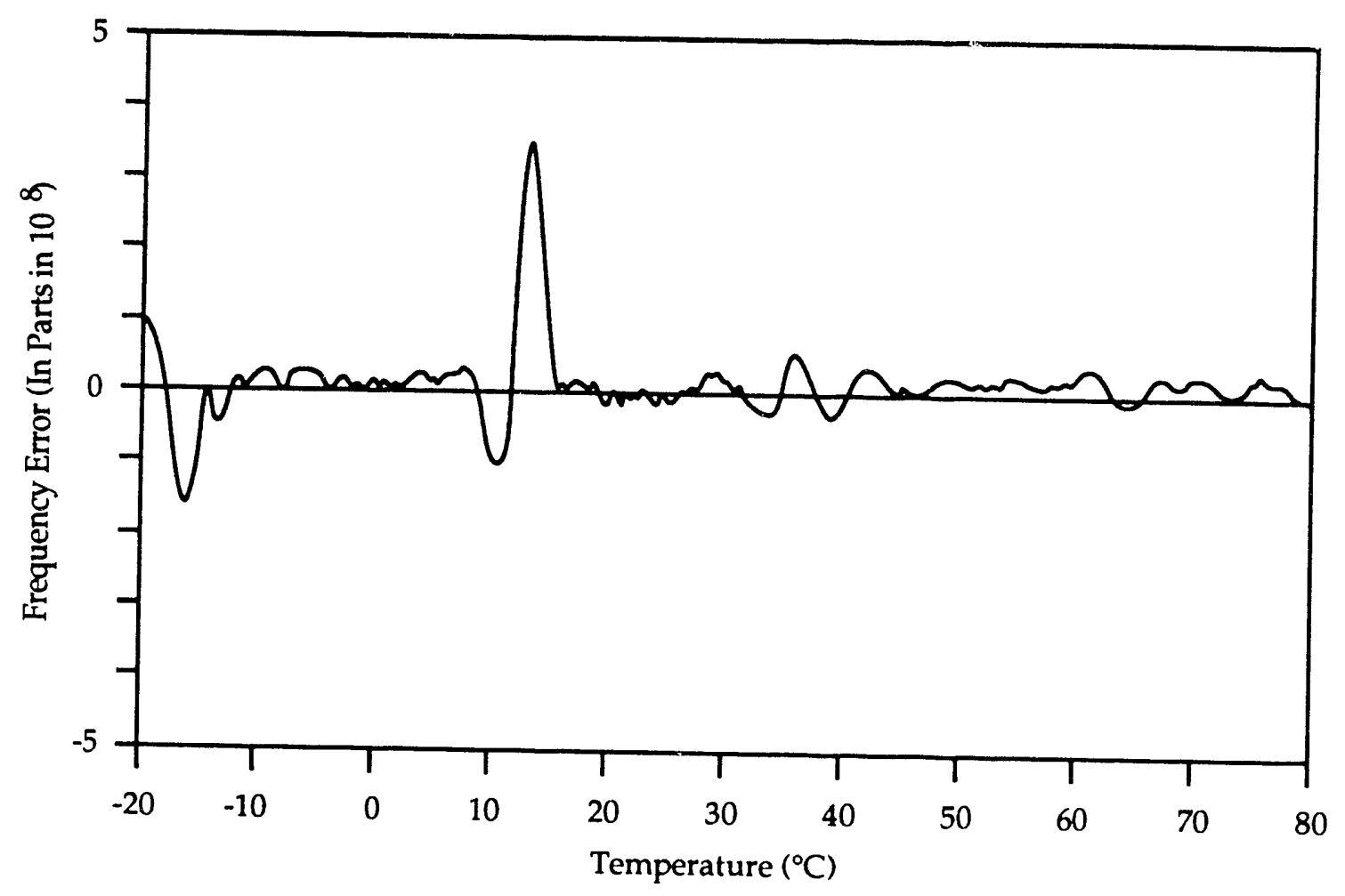

Figure VIII.6

Compensation Error: Quadratic Interpolation, 30 Points

\begin{tabular}{|c|c|c|}
\hline \multicolumn{3}{|c|}{$\begin{array}{c}\text { Table VIII.1 } \\
\text { COMPARISON OF SOVIET AND US OSCILLATORS }\end{array}$} \\
\hline & Bagayev et al., 1986 & Filler et al., 1989 \\
\hline $\begin{array}{l}\text { Size }\left(\mathrm{cm}^{3}\right) \\
\text { Power (milliwatts) } \\
\text { Temperature Range }\left({ }^{\circ} \mathrm{C}\right) \\
\text { Stability Over Temperature } \\
\text { Agirg Rate }\end{array}$ & $\begin{array}{c}100 \\
85 \\
-10 \text { to }+55 \\
\pm 3 \times 10^{-8} \\
\text { Not given }\end{array}$ & $\begin{array}{c}75 \\
41 \\
-55 \text { to }+85 \\
\pm 5 \times 10^{-8} \\
1 \times 10^{-10} / \text { day }\end{array}$ \\
\hline
\end{tabular}




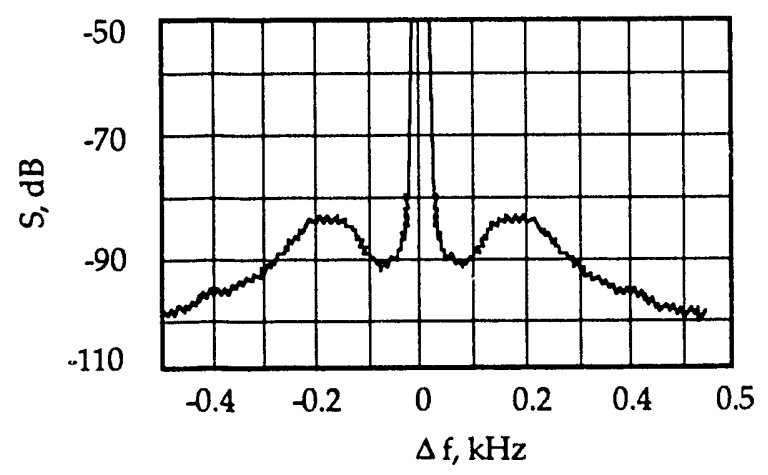

(a)

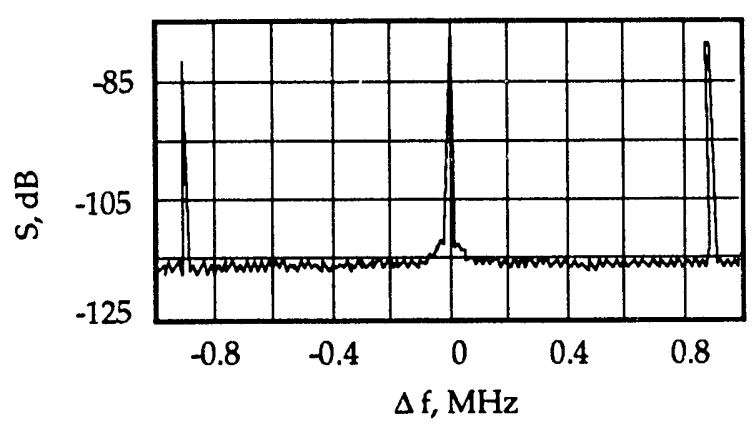

(b)

Figure VIII.7

Noise Increase Around the Carrier

(Bagayev et al., 1986)

\section{SYNTHESIZERS}

While the Soviet literature is awash with patents on synthesizers, there is no mention of the application of these devices to atomic standards. The role of the synthesizer, as shown in the generic example of Figure VIII.8, is to allow the use of an oscillator at a standard frequency in the primary loop while still generating the required atomic resonance frequency. Table VIII.2 lists some common frequencies used in standards.

By design, the synthesizer output frequency is added to the multiplied crystal frequency. This allows the synthesized signal to have a poorer spectral purity without affecting the signal used to interrogate the atomic resonance. Early cesium clocks, such as the Hewlett-Packard 5060A, used a second crystal oscillator to generate the required frequency, but that proved inconvenient because it was sometimes desirable to operate the clock at a frequency that was offset from the calculated nominal. Later Hewlett-Packard clocks included a digital synthesizer whose divisor ratios could be programmed in hardware. The first HewlettPackard 5061A clocks had thumbwheel switches to allow the user to select various preselected offsets to match one of several time scales. As atomic timekeeping matured, it was no longer necessary to make large adjustments to frequency, and the switches were replaced by internal printed circuit connections. 


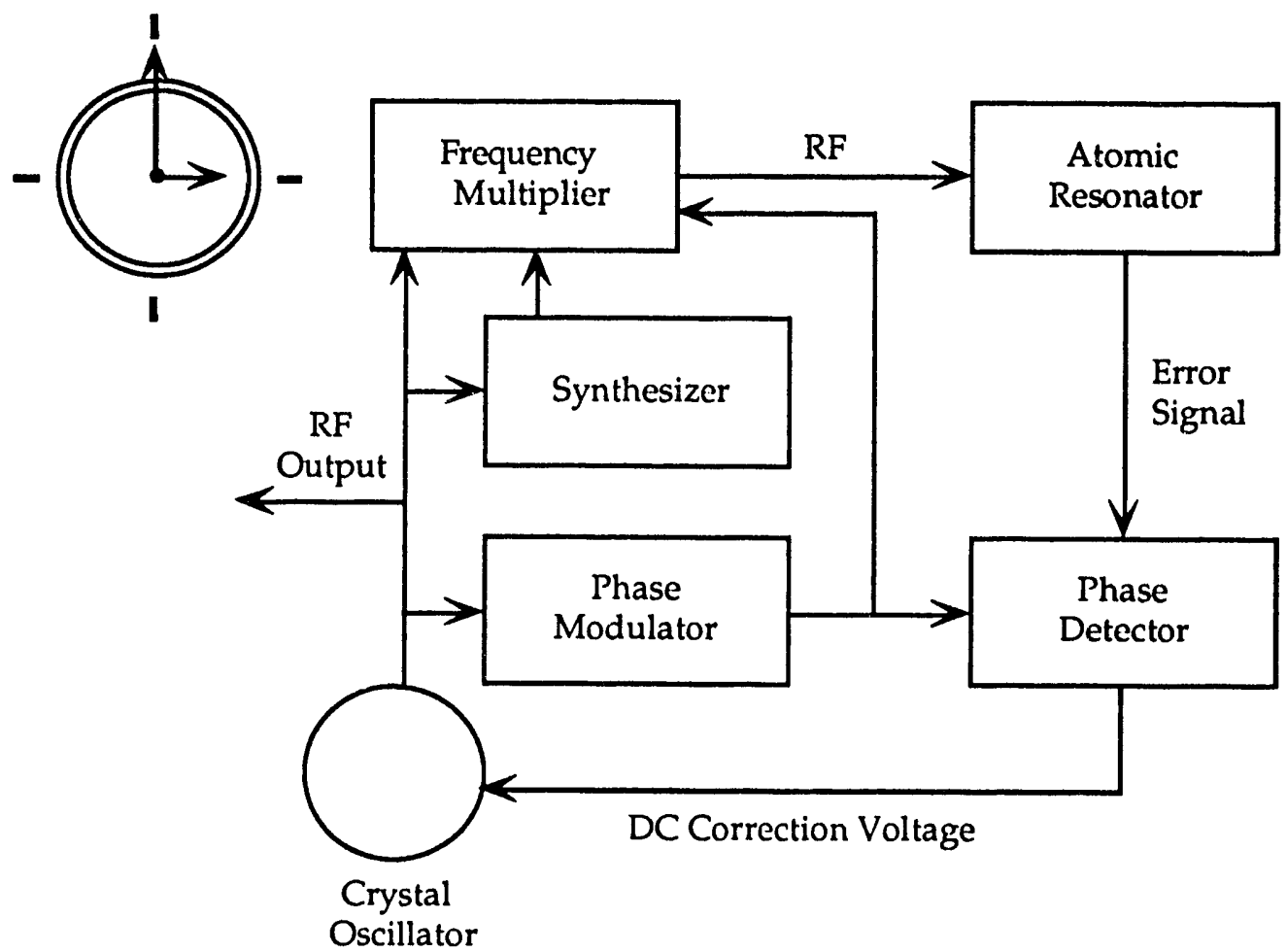

Figure VIII.8

Generic Atomic Clock

\section{Table VIII.2}

COMMON FREQUENCY STANDARDS

\begin{tabular}{|l|c|c|}
\hline Clock Type & Output Frequency & Synthesizer Frequency \\
\hline & & \\
Rubidium & $10.0 \mathrm{MHz}$ & $5.3125 \mathrm{MHz}$ \\
Cesium & $5.0 \mathrm{MHz}$ & $12.631 \mathrm{MHz}$ \\
Cesium & $10.23 \mathrm{MHz}$ & $14.3682 \mathrm{MHz}$ \\
Hydrogen & $5.0 \mathrm{MHz}$ & $.40575168 \mathrm{MHz}$ \\
\hline
\end{tabular}


For many years, the synthesizer was simply a circuit used to create the necessary odd frequency to make the clock work. With the current interest in ensembles of atomic clocks for timekeeping at the nanosecond level, it has again become necessary to make small, precise adjustments in frequency. A device known as a microstepper has been used to adjust the phase of a single clock's output (at $5 \mathrm{MHz}$ ) to correct it to the calculated phase. There are several problems with this approach. The main disadvantage is that the microstepper adds a significant amount of noise to the output spectrum including bright lines at the $100-\mathrm{Hz}$ intervals associated with the stepping rate. Another disadvantage is the addition of yet another electronic box to the system which adds another failure mechanism. Recently, the US Naval Observatory has been correcting the phase of its master clock by adjusting the frequency of one of the hydrogen masers using the maser's internal synthesizer. This method is extremely precise, adds very little noise, and does not add additional devices or complexity to the system. With the appropriate mathematical algorithm, it is now possible to use this method to hold the output phase of a clock to several nanoseconds of a remote reference. The limiting factor now tends to be the precision to which the external signal can be compared with the local signal.

The preferred synthesizer technology for this application is the numerically controlled oscillator (NCO). These oscillators use digital-to-analog converters to create a waveform at the desired frequency from a reference frequency. The Soviet Union appears to use this technique widely and many patent references have been found. The published literature shows several variations on the NCO theme; with some being optimized for lower noise, and others being optimized for higher tuning resolution. Kozlov (1989) describes a device that uses digital phase detectors to control the frequency of a pulse generator with a phase lock loop. The digital output of an accumulator fed from the detectors is put into a digital-to-analog converter to create analog output. The analog output is sampled and used to drive a pulse generator which is monitored by the digital phase detectors. Kozlov's synthesizer is at the current state of the art. There is no indication in the Soviet literature of the tuning range and minimum step size of these NCOs. Western NCOs are capable of 48-bit resolution. A typical hydrogen maser synthesizer using an NCO has a step size of $10^{-8} \mathrm{~Hz}$, which corresponds to a change of about $1 \times 10^{-17}$ in the output frequency. 
A logical application of NCO synthesizers would be in the Soviet Ch0-101B calibrating covice of frequency and time. The Ch0-101B includes two Ch1-80 frequency and time reference standards, each of which contains two hydrogen masers. Also included are two automated measurement systems, a phase comparator, distribution amplifiers, a rubidium clock, and a mechanism for forming working time scales. The time-scale mechanism is quoted as having a resolution of 0.1 microseconds in discrete steps, and a smooth adjustment capability in the 0 to 0.1 microsecond range. This would imply a minimum control of roughly 50 nanoseconds, depending upon their definition of smooth. This device apparently does not have computer control of the output signal. The use of an NCO synthesizer along with an appropriate ensembling algorithm in the internal model 908 microcomputer could turn the Ch0-101B into a nanosecond level system. The addition of a GLONASS receiver to provide a reference to GLONASS time would allow steering of this ensemble to remove the long-term frequency instabilities of the masers. While Soviet researchers apparently have these tools in hand, there is no evidence that they have actually applied them to operate such a system.

\section{FREQUENCY MULTIPLIERS}

Within atomic standards, frequency multipliers are essential for creating the necessary microwave signals to interrogate the resonance. Ideally, frequency multipliers should do nothing but multiply the input signal. In practice, they also add short-term noise, long-term phase drifts, and spurious spectral line components. Relatively few papers on multiplier design are published either in the West or in the Soviet Union.

Early Soviet work by Il'in and Lukin (1972) shows the use of a heterodyne frequency multiplier with effective multiplication up to $10^{4}$. Their frequency stability results of $1.5 \times 10^{-13}$ at 1 second and $1 \times 10^{-14}$ at 100 seconds are not outstanding results for that time. Ivanov (1975) analyzed the effects of short-term instability in the synthesizer. He performed a good theoretical treatment which confirmed the intuitive conclusion that the performance is inferior when the synthesizer is outside the frequency control loop of the standard. Ivanov also showed that optimal loop filtering gives somewhat better performance than simple integration at shorter averaging times. 
The Soviet Union has also used klystrons as microwave signal sources for cesium clocks (Yelkin and Purtov, 1976). A frequency multiplier was used to lock the klystron and to generate square wave frequency modulation. The multiplication factor was 1,836 starting with a $5-\mathrm{MHz}$ source. The instability added by the multiplier was $5 \times 10^{-14}$ in the form of slow phase drifts.

In related work, Basov et al. (1979) described the use of superconductive point contact devices to provide useful frequency multiplication and mixing in the range of $10^{9}$ to $10^{13} \mathrm{~Hz}$ with good noise performance. Lipatov and Sizov (1984) used high-order frequency multiplication to generate a reference signal for a radio astronomy receiver. Here, the $5-\mathrm{MHz}$ output of a rubidium standard was multiplied to $300 \mathrm{MHz}$ using a charge storage diode. The $300 \mathrm{MHz}$ was then applied to another charge storage diode to generate harmonics in the 5.7- to 7.5-GHz range. This design suppressed unwanted harmonics of the $300 \mathrm{MHz}$ by 40 decibels with the lower order spurious signals (for example, $5 \mathrm{MHz}$ ) at negligible levels.

Experimental work was conducted on a metal-oxide-metal diode for harmonic generation in the sub-millimeter and infrared ranges (Klement'yev et al., 1985). The diode consisted of an 8-micron tungsten wire with a 0.5 -micron diameter rounded end pressed against a cobalt surface. The diode was fed with a 2- to $3-\mathrm{GHz}$ drive signal, and harmonic mixing in the diode with a millimeter wave source was investigated. For harmonics $\mathrm{N}$ greater than 15, the signal-tonoise ratio of the diode mixer output was proportional to $\mathrm{N}^{-2}$. Harmonics up to about 30 were usable. The results depended only weakly on the frequencies involved because of the antenna properties of the tungsten wire. Harmonic generation in the submillimeter and infrared range should be achievable with this type of diode. Work conducted much earlier in the United States on metaloxide-metal diodes was referenced.

\section{E. PROJECTIONS FOR THE FUTURE}

The main Soviet disadvantage in the supporting technology area appears to be its lack of high-quality SC-cut quartz crystal resonators. In all other areas, the Soviet Union shows capabilities similar to those of the United States. Since the 
usefulness of this type of crystal cut is well documented in Western scientific and engineering literature, the Soviet Union must be aware that developing its ability to manufacture doubly rotated cut crystals is critical to future progress. 


\section{CHAPTER VIII: AVAILABLE SUPPORTING TECHNOLOGY REFERENCES}

Bagayev (Bagaev), S. N., A. V. Kosykh, A. N. Lepetayev (Lepetaev), and V. F. Samoylenko (Samoilenko), "A Two-Mode Crystal Oscillator with Digital-Thermal Compensation," Telecomm. Radio Eng., 40/41, 6(1986), 44-47.

Basov, N. G., E. M. Belenov, S. I. Vedeneyev (Vedeneev), G. P. Motulevich, and V. V. Nikitin, "Frequency Multiplication in the $10^{10}-$ to $10^{13}-\mathrm{Hz}$ Range with the Aid of Weak Superconductive Couplings," Sov. Phys.-Usp., 22, 8(1979), 662.

Fromberg, E. M., and V. S. Yampolskiy (Iampolskii), "Temperature Regulator for a Crystal Oscillator," Otkrytiya, izobreteniya, 45 (1986), 157.

Il'in, V. G., and A. E. Lukin, "Phase Fluctuations in a Heterodyne Multistage Frequency Multiplier," Meas. Tech., 15, 11(1972), 1659-1661.

Ivanov, N. I., "Short-Term Instability of the Frequency Synthesizer in an Atomic Clock," Meas. Tech., 18, 2(1975), 248-251.

Klement'yev (Klement'ev, Klementiev), V. M., B. A. Timchenko, and V. P. Chebotayev (Chebotaev), "Generation of Higher Harmonics in a Point W-Co Contact," Sov. J. Quantum Electron., 15, 11(1985), 1541-1543.

Kozlov, V. I., "Frequency Synthesizers Based on Storage Adders," Elektrosvyaz, 2 (1988), 53.

Lipatov, B. N., and A. S. Sizov, "A System for the Coherent Conversion of the Signals of the Centimeter-Band Astronomical Very Long Baseline Radio Interferometer of the Scientific Research Institute of Radiophysics," Radiophys. Quantum Electron., 27, 2(1984), 79-82.

Vishina, A. V., and G. A. Yelkin (Elkin), "5-MHz Crystal Oscillator with High Short-Term Stability," Meas. Tech., 28, 1(1985), 56-58.

Yelkin (Elkin), G. A., and V. I. Purtov, "Automatic System for Comparing the Frequency of a Quantum Store with the Transition Frequency of Cesium Atoms," Meas. Tech., 19, 10(1976), 1456-1460. 
(blank)

VIII-20 


\section{CHAPTER IX \\ INSTITUTIONAL CONSIDERATIONS: THE SOVIET PRECISION TIMEKEEPING ESTABLISHMENT}

\section{A. INTRODUCTION}

The development of precision timekeeping technology in the Soviet Union as well as the United States has involved a complex of scientific, industrial, and government institutional organizations. In both countries the elements of the complex include measurement standards laboratories, national academies of science, technology-based government agencies (particularly defense and space), universities, industrial research laboratories, and manufacturing enterprises. While the involvement of counterpart organizations in the two countries may suggest similarities, the details are, in fact, very different. From a general viewpoint, the differences reflect the controlling role of central planning in the Soviet Union and the very different degrees of autonomy and interaction among various institutional organizations. In this connection, the basic driving forces that affect communication and enterprise in the two nations are clearly involved. The purpose of this discussion is to bring some of the more important institutional factors into focus as they affect the progress of modern precision timekeeping technology in the two nations. This discussion is based on panelist A. O. McCoubrey's experience in the program for technical cooperation between the Soviet Union and the United States in the field of metrology,1,2,3 a book describing the organization of science and technology in the Soviet Union, ${ }^{4}$ and information gained from a review of the published Soviet literature. It must be

In 1972, the Unite: $i$ States and the Soviet Union entered into a bilateral Agreement on Cooperation in Fields of Science and Technology. One of the working groups under the agreement was concerned with the field of metrology. The responsible US organization was the National Bureau of Standards (now the National Institute of Standards and Technology); the responsible Soviet organization was the Gosstandart (State Committee for Standards). From 1976 until the termination of cooperation in the field of metrology in 1983, A. O. McCoubrey was Chairman of the US working group. The Soviet Chairman (V. I. Kiparenko, followed by L. Isayev) was the Chief of the Metrology Administration of Cosstandart.

A. O. McCoubrey, R. J. Celotta, D. G. McDonald, J. L. Tech, and R. D. Young, Foreign Trip Report (16 May-3 June 1976, USSR), National Bureau of Standards, Washington, DC, 1976. Report (24 June-6 July 1979, USSR), National Bureau of Standards, Washington, DC, 1979. 
emphasized that the first two sources involve information that is now quite old, and the third source involves information that tends to be speculative. This discussion of institutional considerations does not reflect the remarkable developments that have been unfolding in the Soviet Union; these developments will (if they have not already) induce major changes in Soviet institutions and their interrelationships.

Modern precision timekeeping was motivated by scientific advances in radio frequency spectroscopy just before and after World War II. The earliest advances were made in universities, and major contributions were made during the same time period in the Soviet Union and the United States. These parallel scientific advances are very much reflected by the award of the 1964 Nobel Prize in Physics to Charles Townes of the United States and to Nikolay Basov and Aleksandr Prokhorov of the Soviet Union. In the Soviet Union and the United States, scientists were well aware of the relevance of their discoveries to precision timekeeping. In the 1950s, many US scientists (Zacharias, Dehmelt, Ramsey, Dicke, and others) became involved in practical developments in cooperation with industrial laboratories and the government. This industrial work was substantially supported by the Department of Defense and NASA. The first practical approach to precision timekeeping, based on microwave absorption in ammonia, was undertaken at the National Bureau of Standards (now the National Institute of Standards and Technology) in 1948. This approach was followed by ammonia maser studies and cesium atomic beam development in the early 1950s. The first commercial cesium atomic beam frequency standards were delivered in 1957, and hydrogen masers were introduced in the early 1960 s.

In the Soviet Union, the first practical use of quantum phenomena for precision timekeeping appears to have begun in 1959, under the technical leadership of A. Ya. Leykin. This work apparently involved the ammonia maser (Belotserkovskiy and Paliy, 1972). In 1962, a "molecular generator" (probably an ammonia maser) was used by G. A. Yelkin at the State Time Service Laboratory (VNIIFTRI, see discussion below). The hydrogen maser was first introduced in 1967, and the first cesium atomic beam frequency standard was operated in 1976. The first evidence of the direct involvement of Soviet scientists was at the First All-Union Symposium on Quantum Measures of Frequency in 1980, where academician Basov was the chairman of the Organizing Committee. 
Historical accounts of precision timekeeping developments in the United States are given by Ramsey 5 and Forman. ${ }^{6}$ A sketchy historical account of the developments in the Soviet Union is given by Belotserkovskiy and Paliy (1972).

There are fundamental differences between the Soviet Union and the United States that affect the practical advancement of precision timekeeping in each country. In the United States, there are relatively frequent and open communications between academic and industrial scientists. These scientists receive incentives (financial rewards) to become involved in collaborative work with industry. In addition, US government agencies, such as the Department of Defense and NASA, encourage investment in industrial capacity to use new technology.

There is an apparent absence of these factors in the Soviet Union. The Soviet scientific establishment relies excessively upon bureaucratic central plans developed at levels of government that are far removed from the appreciation of science, applications, and new opportunities.

The following sections discuss the various Soviet institutional organizations involved in the development of precision timekeeping.

\section{B. SOVIET MANAGEMENT OF SCIENCE AND TECHNOLOGY}

The Soviet system for managing science and technology is complex, appears to be changing in real time, and a complete description of it is well beyond the scope of this report. Therefore, the following discussion is limited to the level of Sovi st government that is concerned with managing the major institutional organizations involved in precision timekeeping. A more detailed discussion of this topic can be found in a 1988 book that describes the management structure of Soviet science and technology after two years of Gorbachev's rule. ${ }^{4}$

$5 \quad$ N. F. Ramsey, "History of Atomic Clocks," J. Res. Nat'l. Bur. Stand., 88 (1983), 301.

6 P. Forman, "Atomichron: The Atomic Clock from Concept to Commercial Product," Proc. IEEE, 73 (1985), 181. 
At that time, it was clear that the Communist Party was the highest level of Soviet government and was ruled through the Council of Ministers. The situation is currently fluid; there is now a Presidential Council that appears to be Gorbachev's equivalent of a cabinet of ministers, and the Communist Party of the Soviet Union is torn with dissention. Within this framework, there are at least three Union-level departments of interest for this discussion.

The State Standards Committee (Gosstandart), the Soviet Academy of Sciences, and the Ministry of Education are directly involved in precision timekeeping technology. It is likely that at least one industrial ministry is involved, but specific information was not available. It must be recognized that the Ministry of Defense is involved; however, direct evidence of this is limited (Kalaytanov and Boriskin, 1984). Recent prospecti (December 1990) from the Leningrad Radiotechnical Scientific Research Institute indicate that this institution is heavily involved in space and military work.

\section{GOSSTANDART (STATE COMMITTEE FOR STANDARDS)}

Gosstandart is an immense and bureaucratic Soviet organization (Boytsov, 1975) that consists of two major structures: the Standardization Administration, and the Metrology Administration.

It is difficult to estimate the size of Gosstandart, but in a conversation with a US researcher, the Soviet Chief of the Metrology Administration once claimed that his administration alone employed 250,000 people throughout the Soviet Union. The corresponding employment in the same activities in the United States is almost certainly less than 20,000 people (the National Institute of Standards and Technology employs 3,000 people).

\section{The Standardization Administration}

The Gosstsandart Standardization Administration is roughly comparable to US organizations that include all private sector standards writing bodies (ASTM, ASME, API, IEEE, and EIA), the American National Standards Institute (ANSI, the coordinating body), all related publishing activities, all private sector product testing laboratories (Underwriters Laboratories), and all related regulatory bodies. 
The Standardization Administration of Gosstandart is described by Boytsov (1975), but the details are not particularly applicable to this discussion of precision timekeeping technology. It is relevant to note that elaborate support is provided for a very large body of product standards, design standards, process standards, and testing standards. The expansion of these standards and their effective application to industrial quality assurance has been the focus of important objectives in the five-year plans of the Soviet government. In contrast to traditional US practice, formal standards (designated by GOST numbers) are enforced by the Soviet State for quality control of products, as well as for regulation of public health and safety. In the United States, prevailing practice favors, to a much greater extent, an emphasis on product performance standards in preference to product design standards; the driving force in this connection is the tendency of product design standards to inhibit innovation.

\section{The Metrology Administration}

The Gosstandart Metrology Administration is roughly comparable to the US National Institute of Standards and Technology (NIST) in combination with all state and local weights and measures laboratories and all private sector industrial calibration service laboratories. Gosstandart has branch organizations in all the republics and autonomous regions of the Soviet Union.

The Metrology Administration of Gosstandart includes the three special scientific institutes described below.

\section{a. VNIIMS (Metrological Services All-Union Scientific Research Institute)}

VNIIMS, headquartered in Moscow, is responsible for solving special scientific organizational and methodological problems that are important to the effectiveness of the metrological services.

In the early 1970s, a high-level research laboratory was organized as part of VNIIMS; the nucleus of the staff was a small group of very competent scientists from the High Temperatures Institute of the Soviet Academy of Sciences. A ded- 
icated laboratory, located next to the new headquarters of the Soviet Academy of Sciences in Moscow, was established with unusual (for Gosstandart) priority access to hard currency for equipment. Scientific research at this laboratory focused mainly on basic work leading to new standard reference data. However, in 1979, there was also some preliminary work on measuring the electromagnetic radiation in the environment.

The organization of this special research laboratory in VNIIMS reflects a high-level Soviet government decision to improve the scientific capabilities of Gosstandart, and the Soviet Academy of Sciences was a major factor in its implementation. The potential of this laboratory to contribute to precision timekeeping technology was certainly high, but visits to VNIIMS in 1976 and 1979 by A. O. McCoubrey revealed no evidence of any related activity. In fact, in 1979, there seemed to be some policy barriers affecting decisions to work in electromagnetic technology areas within the mission of the Gosstandart institute responsible for precision timekeeping (but there was no evidence of any direct effect on precision timekeeping).

b. VNIIM (Metrology All-Union Scientific Research Institute im. D. I. Mendeleyev)

VNIIM is headquartered in Leningrad in the historical Mendeleyev Institute of Metrology. This establishment was founded in 1842 to provide national reference standards for weights and measure. In 1893, under the leadership of D. I. Mendeleyev, 7 the institute became one of the first scientific metrology laboratories to address the needs of emerging electrical technology and the accelerating industrial revolution. The corresponding scientific metrology laboratories in other industrial nations included the Physikalisch-Tecknische Reichsanstalt (now the Physikalisch-Tecknische Bundesanstalt [?TB]) in Germany (1887), the National Physics Laboratory in Great Britain (1899), and the National Bureau of Standards (now the National Institute of Standards and Technology) in the United States (1901). The Mendeleyev Institute, as a component of Gosstandart, introduction (in 1867) of the Periodic Table of the Elements. He also made pioneering contributions to Russian industry (petroleum, coal, gunpowder, and shipbuilding). 
continues to have a high level of institutional dignity and historical pride. The responsibilities of the Mendeleyev Metrology Institute include mass, length, temperature, pressure, force, optical quantities, electrical quantities, magnetic quantities, ionizing radiation, and neutron flux. These activities essentially correspond to the metrology programs of the US National Institute of Standards and Technology in Gaithersburg, Maryland.

A relatively new (1977) Branch Laboratory of VNIIM is located in Lomonosov, about 40 kilometers northwest of Leningrad. There are additional branches of VNIIM in other parts of the Soviet Union, including Sverdlovsk and Tbilisi.

Visits to VNIIM branches in Leningrad, Lomonosov, and Tbilisi in 1976 and 1979 revealed that the laboratory equipment at VNIIM was mediocre and relatively out of date (as much as 10 years), compared to equipment in corresponding laboratories at the US National Institute of Standards and Technology.

At the Mendeleyev Metrology Institute, work on length standards based on lasers stabilized by iodine and methane absorption cells is also linked to precision timekeeping (Bagayev and Chebotayev, 1986; Korobov et al., 1984). In this connection, it was clearly recognized in the Soviet Union, as well as in other countries, that the frequency of well-stabilized lasers could be accurately determined by comparison with the national standard of frequency (cesium atomic beam standard). To carry out this comparison, scientists at the Mendeleyev Metrology Institute collaborated with the Gosstandart precision timekeeping laboratory in Moscow (see discussion below). Their results, published in 1984, are similar to the same comparison first carried out at the National Bureau of Standards about 10 years earlier.

c. VNIIFTRI (Physical-Technical and Radio Engineering Measurements All-Union Scientific Research Institute)

VNIIFTRI has five technical divisions: time and frequency, electromagnetics, mechanical measurements, heat, and ionizing radiation. VNIIFTRI is headquartered at Zelenograd in the northern suburbs of Moscow. It has a staff of roughly 2,700 people, about half of which belong to a Design Bureau (see discus- 
sion below). VNIIFTRI was created by restructuring a number of related activities in 1955 (Belotserkovskiy and Paliy, 1972).

Visits to VNIIFTRI laboratories in 1976 and 1979 by A. O. McCoubrey revealed that the laboratory equipment at VNIIFTRI (most of which was manufactured in the Soviet Union) tended to be out of date, compared to equipment in corresponding US laboratories.

The time and frequency and electromagnetics programs at VNIIFTRI correspond to programs in the same technical areas at the US National Institute of Standards and Technology laboratories in Boulder, Colorado.

VNIIFTRI has a number of branch laboratories located at Irkutsk, Kazan', Khabarovsk, and L'vov. The Irkutsk Branch appears to be one of the more important locations for precision timekeeping activities, probably because this location has a relatively high-power time and frequency transmitter and an astronomical observatory (affiliated with a local university) that was formerly an astronomical time service facility.

The main laboratory of the Soviet State Time and Frequency Service is located at VNIIFTRI. However, the State Time and Frequency Service appears to be an autonomous entity that is managed by the Interdepartmental Commission for Unified Services of Gosstandart (Boytsov, 1975). The Commission includes representatives from the Soviet Academy of Sciences and a number of ministries (such as the Ministry of Communication) and other departments.

The primary cesium atomic beam frequency standards of the Soviet National Time and Frequency Standard are maintained at VNIIFTRI (Abashev et al., 1989). These resium standards are used in conjunction with an ensemble of hydrogen masers (Abashev et al., 1987).

Within Gosstandart there are formal administrative procedures for the approval and certification of State Standards for measurements (Yelkin et al., 1984). Details and data concerning candidate standards developed in the laboratories of the Metrology Administration must be examined at the highest levels of management, and the final designation of a "State Standard" requires the formal 
action of the Chairman. At the US National Institute of Standards and Technology, the corresponding decisions are made by scientific managers two or three levels below the level of the Director. There is no evidence that the Soviet procedures provide greater quality assurance.

The Soviet State Standard for Time and Frequency is a complex of many individual frequency standards that changes as the technology advances. In 1976, the complex included hydrogen masers and quartz oscillators (Yelkin et al., 1984). The cesium atomic beam frequency standard that was first operated at VNIIFTRI in 1975 was later designated as MTs- 1 and added to the complex. At a later date, an additional cesium atomic beam frequency standard, the MTs-2, was added to the complex, and in 1984 the MTs-3 was also included (Abashev et al., 1986).

\section{SOVIET ACADEMY OF SCIENCES}

The present Soviet Academy of Sciences evolved from the Imperial Academy of Sciences founded in 1724. However, its role since the 1917 revolution has shifted toward increasing policy-related responsibilities. Like the US National Academy of Sciences, the Soviet Academy of Sciences has members who are selected on the basis of their achievements in science; but unlike the US Academy, the Soviet Academy operates more than 200 scientific institutes, laboratories, observatories, experimental stations, libraries, museums, and research ships. The Soviet Academy of Sciences is also affiliated with science academies in the Soviet republics; the organizationai structure tends to be similar in each case. The institutes of the Soviet Academy of Sciences generally are focused on a particular scientific or technical discipline and generally are the leading organizations in the nation in their particular fields.

Institutes of the Soviet Academy of Sciences that contributed to Soviet progress in precision timekeeping are described below.

\section{Physics Institute im. P. N. Lebedev, AS USSR, Moscow}

N. G. Basov has been a member of the Lebedev Physics Institute since 1950, and his publications include a thorough analysis of the design of hydrogen masers (Basov et al., 1968). Other members of the Lebedev Physics Institute also 
regularly make important contributions to precision timekeeping; a recent example is concerned with diode lasers that are essential to the next generation (optically pumped) cesium atomic beam standards (Akulshin et al., 1989).

\section{Thermal Physics Institute, Siberian Branch AS USSR, Novosibirsk}

V. P. Chebotayev, a leading expert on stabilized lasers works at this institute (Bagayev and Chebotayev, 1986). Some very competent work leading to the synthesis of laser frequencies from radio frequencies stabilized by a rubidium frequency standard has occurred at this institute (McCoubrey, 1976). A metal-onmetal whisker diode was used following the work of the US National Bureau of Standards in Boulder, Colorado. Chebotayev continued this interest through the years, and published values for the absolute frequency of a methane-stabilized helium-neon laser (Bagayev et al., 1987). He also suggested the possibility of incorporating an optical frequency standard into the State Standard for Frequency and Time, and claims (perhaps extravagantly) to have operated the first "optical clock" (Bagayev and Chebotayev, 1986; Chebotayev et al., 1982). Continuing in this direction, Chebotayev and his colleagues recently made a direct comparison of optical and hydrogen maser frequency standards (Bagayev et al., 1989). Chebotayev, a frequent visitor to the United States, is a competent and visionary scientist with a long-standing interest in advancing precision timekeeping technology through applications of his research.

\section{Spectroscopy Institute, AS USSR, Troitsk (Kransnaya Pakhra)}

V. S. Letokhov is the leading scientist at this institute. He is working on laser cooling of atoms (Balykin et al., 1985) in programs similar to those in progress at the US National Institute of Standards and Technology in Gaithersburg, Maryland, and in Boulder, Colorado (and a number of other laboratories throughout the world). Soviet and US scientists recognize that a future major scientific advance in precision timekeeping will be based on atoms or molecules that essentially are at rest (thermal motion absent). The most recent publication by Letokhov and his colleagues reflects a fundamental theoretical and experimental investigation of the science that must be understood before a new approach to precision timekeeping can be based on atomic particles that are at 
rest in space (Balykin et al., 1989). Letokhov also travels frequently outside the Soviet Union.

The Soviet Academy of Sciences certainly has contributed to the scientific development of the Gosstandart Metrology Administration. It is quite likely that the academy was instrumental in the involvement of N. G. Basov as the organizer of at least one All-Union Symposium on improving the precision of quantum frequency standards.

\section{E. UNIVERSITIES AND POLYTECHNICAL INSTITUTES}

The teaching staff and students at Soviet universities and polytechnic institutes contribute to research and development. The traditional Soviet bias toward theoretical science seems to be stronger in the universities than in the Academy institutes and, perhaps for this reason, the universities do not seem to be as well equipped for experimental laborato: $y$ research leading toward new technologies. Many academic scientists seem to hold joint appointments with Academy institutes and universities, and their experimental research results tend to credit the institutes. For these reasons and possibly others, no literature was found on precision timekeeping that was clearly based on research in the highest level academic institutions. This situation, to the extent that it is correct, stands in sharp contrast to the productive output of US universities.

In the case of polytechnic institutes, single-curriculum applied institutes, and engineering institutes, the conditions appear to be quite different; research and development is carried out, most of which is funded by contracts from industry. Thus, it is not surprising that contributions to precision timekeeping are published from the Engineering Physics Institute in Moscow (Yegorov and Maslov, 1983, 1984) and the Polytechnic Institute im. M. I. Kalinin in Leningrad (Gornyy et al., 1984, 1987). These publications reflect positive contributions to precision timekeeping. It is also significant to note that the engineering institutes are sources of professional staff for organizations engaged in precision timekeeping development and applications. Again, the corresponding role of engineering schools does not exist to any great extent in the United States, at least, as far as precision timekeeping is concerned. On the other hand, the physics departments of universities are very much involved. 


\section{F. DESIGN BUREAUS}

Design bureaus are unique creations of the Soviet system. They are always associated with a source of science or special technology and they function to incorporate that science or technology into useful prototype products, special materials, or services that are useful to society. The management of a design bureau is generally independent of its affiliated scientific partner. They are staffed with competent applied scientists and engineers.

One design bureau that is important to Soviet precision timekeeping is affiliated with VNIIFTRI and is located in the same complex. This design bureau has been instrumental in the design of hydrogen maser frequency standards that are used on a much greater scale in the Soviet Union than in the United States. Another example is the Special Design Bureau for Analytic Technological Equipment in Uzhgorod. Investigators at this organization have considered the possibility of using the ytterbium atom as a basis for a quantum frequency standard in the visible spectrum (Goldovskaya et al., 1982). It is possible that one or more additional design bureaus are involved in Soviet precision timekeeping; in particular, the advanced state of development of rubidium optically pumped frequency standards suggests that some special arrangements have been made, possibly in Gor'kiy.

Soviet design bureaus play a role that in the United States is played by corporate research laboratories, contract research and development enterprises, and some not-for-profit organizations (such as Arthur D. Little, Battelle Memorial Institute, and SRI).

\section{G. SCIENTIFIC PRODUCTION ASSOCIATIONS}

The scientific production association (NPO) is another unique creation of the Soviet technology system. It functions to create and apply technology in the shortest possible time and to introduce advanced products to users on a pre-production basis. The organization of an NPO varies from case to case, but in general, it seems to be superimposed upon a number of existing organizations that have the capacity to create technology and to produce products on a small scale. 
VNIIM (Gosstandart) in Leningrad was reorganized within an NPO in 1977. Since then, the entity as a whole has been known as NPO VNIIM with an assignment, in addition to the traditional metrological mission responsibilities of the Mendeleyev Metrology Institute, to produce and sell instruments based on Mendeleyev Institute technology for:

- mechanical measurement;

- electromagnetic measurement;

- temperature and thermophysical measurement;

- physicochemical measurement;

- ionizing radiation measurement; and

- hydrophysical measurement.

NPO VNIIM is now encouraged to sell these instruments in hard currency markets.

It is not clear (as of early 1990) whether or not NPOs are an important factor in precision timekeeping technology in the Soviet Union. It is quite possible that the production of hydrogen masers and rubidium frequency standards in Gor'kiy involves one or more NPOs. More insight may be available after a more complete study of information obtained during a recent visit to Gor'kiy by a scientist from the National Institute of Standards and Technology. 8

In December 1990, we became aware of a high level of activity in the development and production of space-worthy rubidium, cesium, and hydrogen clocks at the Leningrad Radiotechnical Scientific Research Institute. The relationship of this institution to the Soviet institutional system is, as yet, unclear.

8 Dr. Jon T. Hougen visited the Microwave Spectroscopy Laboratory at the Applied Physics Institute of the Soviet Academy of Sciences in Gor'kiy on 25-29 September 1989. During this visit, he met with A. A. Ul'yanov, N. A. Demidov, and B. A. Sakharov of the Gor'kiy R\&D Instrument Making Institute, where he was shown an apparently well-designed, compact, commercial-grade hydrogen maser. Dr. Hougen was told that the Soviet engineers were given permission to open direct contacts with their Western counterparts without formal approval from Moscow. They were also encouraged to explore possibilities for commercial sales, and their meeting with Dr. Hougen was the first step in this direction. Dr. Hougen has referred them to David Allan of the NIST Time and Frequency Division. The organizational structure above the Gor'kiy R\&D Instrument Making Institute is not yet clear. 
(blank)

IX-14 


\title{
CHAPTER IX \\ INSTITUTIONAL CONSIDERATIONS: THE SOVIET PRECISION \\ TIMEKEEPING ESTABLISHMENT \\ REFERENCES
}

\begin{abstract}
Abashev, Yu. G., L. P. Yelkina (Elkina), and S. B. Pushkin, "The Main Characteristics and the Results of Long-Term Comparisons Between Primary Cesium Beam Standards and Ensemble of Hydrogen Clocks of the National Time Standard," IEEE Trans. Instrumentation and Measurement, IM-36, 2(1987), 627-629.
\end{abstract}

Abashev, Yu. G., V. N. Baryshev, G. A. Yelkin (Elkin), and Y. N. Yakovlev (Iakovlev), "Metrological Parameters of the Primary Cesium Beam Frequency Standards in the National Time and Frequency Standard of the USSR," Proc. IV Symp. on Frequency Standards and Metrology, Berlin: Springer-Verlag, 1989.

Abashev, Yu. G., V. N. Baryshev, G. A. Yelkin (Elkin), Yu. V. Timofeyev (Timofeev), L. I. Polyakova (Poliakova), S. B. Pushkin, V. Yu. Runov, and A. A. Ul'yanov, "Metrological Analysis of Type MTs-3 Cesium Frequency Reference," Meas. Tech., 29, 2(1986), 97-98.

Akulshin, A. M., V. V. Nikitin, V. A. Sautenkov, V. L. Velichanskiy (Velichanskii), E. K. Yurkin (Iurkin), and A. S. Zibrov, "Frequency Stabilization of Highly Coherent AlGaAs Diode Lasers," Proc. IV Symp. on Frequency Standards and Metrology, Berlin: Springer-Verlag, 1989, 236-241.

Bagayev (Bagaev), S. N., V. M. Klement'yev (Klement'ev, Klementiev), and V. P. Chebotayev (Chebotaev), "Measurements of the Absolute Frequency of an $\mathrm{He}-\mathrm{Ne} / \mathrm{CH}_{4}$ Laser," JETP Lett., 45, 2(1987), 83-86.

Eagayev (Bagaev), S. N., and V. P. Chebotayev (Chebotaev), "Laser Frequency Standards," Sov. Phys.-Usp., 29, 1(1986), 82-103.

Bagayev, S. N., V. P. Chebotayev (Chebotaev), V. M. Klement'yev (Klement'ev, Klementiev), B. A. Timchenko, and V. F. Zakharash, "Direct Comparison of the Characteristics of Optical and Hydrogen Frequency Standards," Proc. IV Symp. on Frequency Standards and Metrology, Berlin: Springer-Verlag, 1989.

Balykin, V. I., et al., "Recent Programs in the Control of the Motion of Atomic Beams by Laser Fields," Proc. IV Symp. on Frequency Standards and Metrology, Berlin: Springer-Verlag, 1989, 248263.

Balykin, V. I., V. S. Letokhov, and V. G. Minogin, "Cooling of Atoms by Means of Laser Radiation Pressure," Sov. Phys.-Usp., 28, 9(1985), 803-826.

Basov, N. G., G. M. Strakhovskiy (Strakhovskii), V. V. Nikitin, T. F. Nikitina, V. M. Tatarenkov, and A. V. Uspenskiy (Uspenskii), "Design Problems and Investigation of the Performance of the Hydrogen Atomic Beam Quantum Oscillator," Quantum Electronics in Lasers and Masers, Proc. (Trudy) of the P. N. Lebedev Physics Institute, 31 (1968), 127-161.

Belotserkovskiy (Belotserkovskii), D. Yu. and G. N. Paliy (Palii), "State Time and Frequency in the USSR," Meas. Tech., 15, 12(1972), 1804-1811. 
Boytsov (Boitsov), V. V., Standardization in the USSR: 1925-1975, Moscow: Standartov, 1975 (translated from Russian and published for the National Bureau of Standards and the National Science Foundation by Amerind Publishing Co. Pvt., Ltd., New Delhi, 1980).

Goldovskaya (Goldovskaia), N. V., V. L. Goldovskiy (Goldovskii), L. L. Shimon, and I. I. Dudich, "Possibility of Establishment of a Quantum Frequency Standard for the Visible Range Using an Intercombination Spectral Transition in the Ytterbium Atom," Sov. J. Quantum Electron., 12, 12(1982), 1659.

Gornyy (Gornyi), M. B., B. G. Matisov, G. M. Smirnova, and V. I. Khutorshchikov, "Short-Term Stability of Rubidium Frequency Standards," Sov. Phys.-Tech. Phys., 32, 4(1987), 448-452.

Gornyy (Gornyi), M. B., N. A. Dovator, R. A. Zhitnikov, and B. G. Matisov, "Spatial Inversion of the Population Difference of Hyperfine Sublevels of the Ground State of Cesium Atoms During Optical Pumping," Sov. Tech. Phys. Lett., 10, 1(1984), 15-16.

Kalaytanov, V., and Ye. Boriskin, "Frequency and Time Standard Ch1-74," Technology and Armaments, 11 (1984), 45.

Korobov, V. K., Yu. V. Tarbeyev (Tarbeev), N. P. Gerasimov, and S. B. Pushkin, "Single TimeFrequency-Length Standard," Meas. Tech., 27, 7(1984), 579-582.

Yegorov (Egorov), V. K., and V. A. Maslov, "Calculation of the Efficiency of Optical Pumping of ${ }^{133}$ Cs by Monochromatic Radiation," Sov. J. Quantum Electron., 13, 12(1983), 1635-1636.

Yegorov (Egorov), V. K., and V. A. Maslov, "Effect of Light Shifts on the Accuracy of Optically Pumped Cesium Atomic-Beam Frequency Standards," Sov. Phys.-Tech. Phys., 29, 3(1984), 334-338.

Yelkin (Elkin), G. A., S. B. Pushkin, and V. M. Tatarenkov, "Government Certified Primary Time and Frequency Reference Standard," Meas. Tech., 7 (1984), 25-26 (in Russian). 


\section{APPENDIX A1 \\ EXCERPTS FROM "PERFORMANCE OF SOVIET AND US HYDROGEN MASERS"}

in

Proceedings of the Twenty-Second Annual Precise Time and Time Interval (PTTI) Applications and Planning Meeting, 1990

by
A. A. Ul'yanov
N. A. Demidov
E. M. Mattison
R. F. C. Vessot
D. W. Allan
G. M. R. Winkier 
(blank)

A-2 


\begin{abstract}
The frequencies of Soviet- and US-built hydroger masers located at the Smithsonian Astrophysical Observatory (SAO) and at the United States Naval Observatory (USNO) were compared with each other and, via GPS common-view measurements, with three pr:mary frequency-reference scales. The best masers were found to have fractional frequency stabilities as low as $6 \times 10^{-16}$ for averaging times of approximately $1 C^{4}$ seconds. Members of the USNO maser ensemble provided frequency prediction better than $1 \times 10^{-14}$ for periods up to a few wceks. The frequency residuals of these masers, after removal of frequency drift and rate of change of drift, had stabilities of a few parts in $10^{-15}$, with several masers achieving residual stabilities well below $1 \times 10^{-15}$ for intervals from $10^{5}$ seconds to $2 \times 10^{6}$ seconds. The fractional frequency drifts of the 13 masers studied, relative to the primary reference standards, ranged from $-0.2 \times 10^{-15} /$ day to $+9.6 \times 10^{-15} /$ day.
\end{abstract}

\title{
MASERS STIJDIED
}

Four of the masers under study were located at the SAO Mäser Laboratory. Two of these, serial numbers P13 and P26, are model VLG-11 masers built by the SAO Maser Group. The Soviet masers at SAO were a model Ch1-75 active maser and a model Ch1-76 passive maser. All masers other than Ch1-76 were active oscillators. Maser Ch1-75 is equipped with an autotuning system designed to stabilize the resonance frequency of the maser's microwave cavity, and thus reduce frequency variations due to cavity pulling. The autotuner, which was operated during part of this study, employs linewidth modulation by means of alteration of the internal magnetic field gradient at intervals of 100 seconds; a high-stability signal from another maser is used as a reference for the system. Masers P13 and P26 do not use cavity autotuners.

Nine masers at the USNO were studied. Three were SAO VLG-11 masers, serial numbers P18, P19, and P22; two were SAO VLG-12 masers, numbers P24 and P25; and four were commercial masers, serial numbers N2, N3, N4, and N5.

The masers were compared with each other and with three primary time scales, TAI, NIST(AT1), and UTC(PTB). TAI (International Atomic Time) is maintained by the Bureau International de Poids de Mesure (BIPM) in Sevres, France; it incorporates time and frequency data from about 180 clocks located in more than 50 standards laboratories throughout the world. NIST(AT1) is an unsteered, unsyntonized cesium-generated time scale maintained by NIST Time and Frequency Division, Boulder, Colorado. Its generating algorithm, AT1, is optimized for frequency stability 
and minimum time prediction error. UTC(PTB), generated at the Physikalische-Technische Bundesanstalt in Braunschweig, Germany, is controlled by PTB primary cesium standard Cs-1.

\section{LONG-TERM DRIFT}

The frequencies of the masers at SAO against NIST(AT1) are shown in Figure 1; their drift rates relative to NIST(AT1), calculated by means of linear regressions on the frequencies over the entire observation period, are given in Table 1. The Ch1-75 - NIST(AT1) frequency difference was obtained from the GPS common-view measurements. The frequencies of P13 and P26 relative to NIST(AT1) were then calculated using the frequency differences between those masers and Ch1-75 measured by the SAO beat frequency measurement system; the values plotted represent one-hour averages observed once per day. Daily measurements of the Ch1-75 - Ch1-76 time difference yielded the data for Ch1-76. The frequency excursions and subsequent recoveries seen in Figure 1 for P13 and P26 at MJD 48201 were due to a two-hour power failure that affected those masers but not Ch1-75 or Ch1-76. With the exception of the P13 and P26 excursions on MJD 48201, fluctuations that correlate among the data for Ch1-76, P26, and P13 are probably due to the GPS common-view time transfer or to NIST(AT1); such fluctuations, which appear across the data length, amount to roughly 1 to 2 nanoseconds per day, which is consistent with the level of previously observed common-view time transfer noise. The fluctuations are absent from the Ch1-75 graph because Ch1-75 was the clock controlling the GPS receiver, and its data were Kalman filtered with respect to NIST(AT1). The correlated fluctuations are difficult to identify in Ch1-76's frequency graph prior to MJD 48200, probably because they are obscured by Ch1-76's frequency variations.

\section{FREQUENCY STABILITY}

Short-term frequency stability measurements of the active masers at SAO, as expressed by the Allan deviation $\sigma_{\mathbf{y}}(\tau)$, were obtained from the beat-frequency measurements system and are shown

in Figure 2. The measure used in Figure 2 is the reduced Allan deviation $\tilde{\sigma}_{y}(\tau)=\sigma_{y}(\tau) / \sqrt{2}$ for the frequency pair. If two oscillators contribute equal amounts of noise, then $\tilde{\sigma}_{y}(\tau)$ represents the frequ ency variability of the individual oscillators; if, however, one oscillator contributes considerably more noise than the other, as in the case of Ch1-75 with autotuner on, then $\sigma_{y}(\tau)$, rather than $\tilde{\sigma}_{y}(\tau)$, represents the variability of the noisier oscillator. Figure 2 gives $\sigma_{y}(\tau)$ for the frequency differences P26 - Ch1-75 and P26 - P13 over two 9-day intervals. During interval A, 11 October-20 October (MJD 48175 - 48220), the autotuner was turned off. By the beginning of interval B, P13 and P26 had restabilized following the power interruption on 6 November. Curves $a$ and $b$ in Figure 2 sho' the stability of P26 vs. Ch1-75 with the autotuner on and off (during intervals A and B), 
respectively. A relative drift of $1.68 \times 10^{-14}$ / day has been removed from curve $a$, and a drift of 1.24 $\times 10^{14} /$ day from curve $b$. The peak in curve $a$ at approximately $\tau=100$ seconds probably results from modulation of Ch1-75's internal magnetic field at a period of 100 seconds for the autotuner. Curve $c$ is the reduced Allan deviation for P26 - P13 during interval B; a relative drift of $6.45 \times 10^{-15} /$ day has been removed. The drift-removed stability of P26 - P13 during interval A was not significantly different from curve $c$. The effect of removing frequency drift is seen by comparison with curve $d$, which gives the reduced Allan deviation for P26 - P13 without drift removal. Curves $b$ and $c$ show that the drift-removed stability levels of the Soviet (autotuner off) and SAO masers are comparable for averaging times between a second and a day.

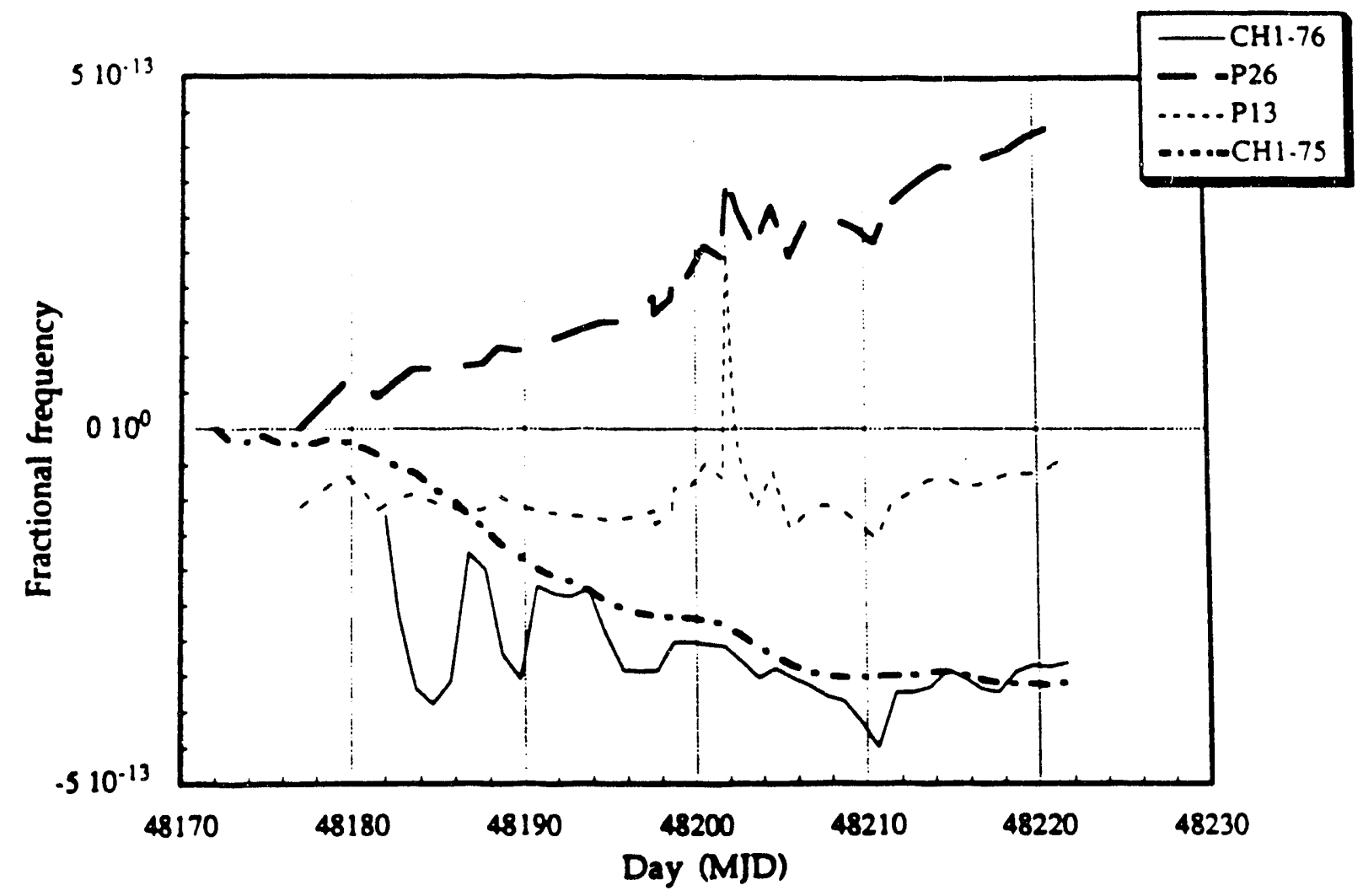

Figure 1

Frequencies of Masers at SAO vs. NIST(AT1)

Comparison of $\tilde{\sigma}_{y}(\tau)$ with the line segments included in Figure 2 shows that $\tilde{\sigma}_{y}(\tau)$ [and thus $\left.\sigma_{y}(\tau)\right]$ is proportional to $\tau^{-1}$ for $\tau<100$ seconds and to $\tau^{-1 / 2}$ for $100<7 \times 10^{3}$ seconds; as predicted theoretically, this behavior is due to additive noise in the maser receivers and noise within the atomic linewidth, respectively. For $\tau>10^{4}$ seconds, $\tilde{\sigma}_{y}(\tau)$ is proportional to $\tau^{1}$ for P26 - P13 with drift not 
removed; this form of $\tilde{\sigma}_{y}(\tau)$ is characteristic of linear frequency drift. In the case of the driftremoved data, portions of the graphs are approximately proportional to $\tau^{1 / 2}$, which is identified with random walk of frequency; this behavior may result from the simultaneous action of several quasi-independent frequency-determining mechanisms in the masers.

Table 1

DRIFT RATES OF MASERS AT SAO VS. NIST(AT1)

\begin{tabular}{|c|c|}
\hline Maser & Frequency Drift $\left(1 \times 10^{-15} /\right.$ day $)$ \\
\hline Ch1-75 & -8.6 \\
Ch1-76 & -3.1 \\
P13 & +0.7 \\
P26 & +9.6 \\
\hline
\end{tabular}

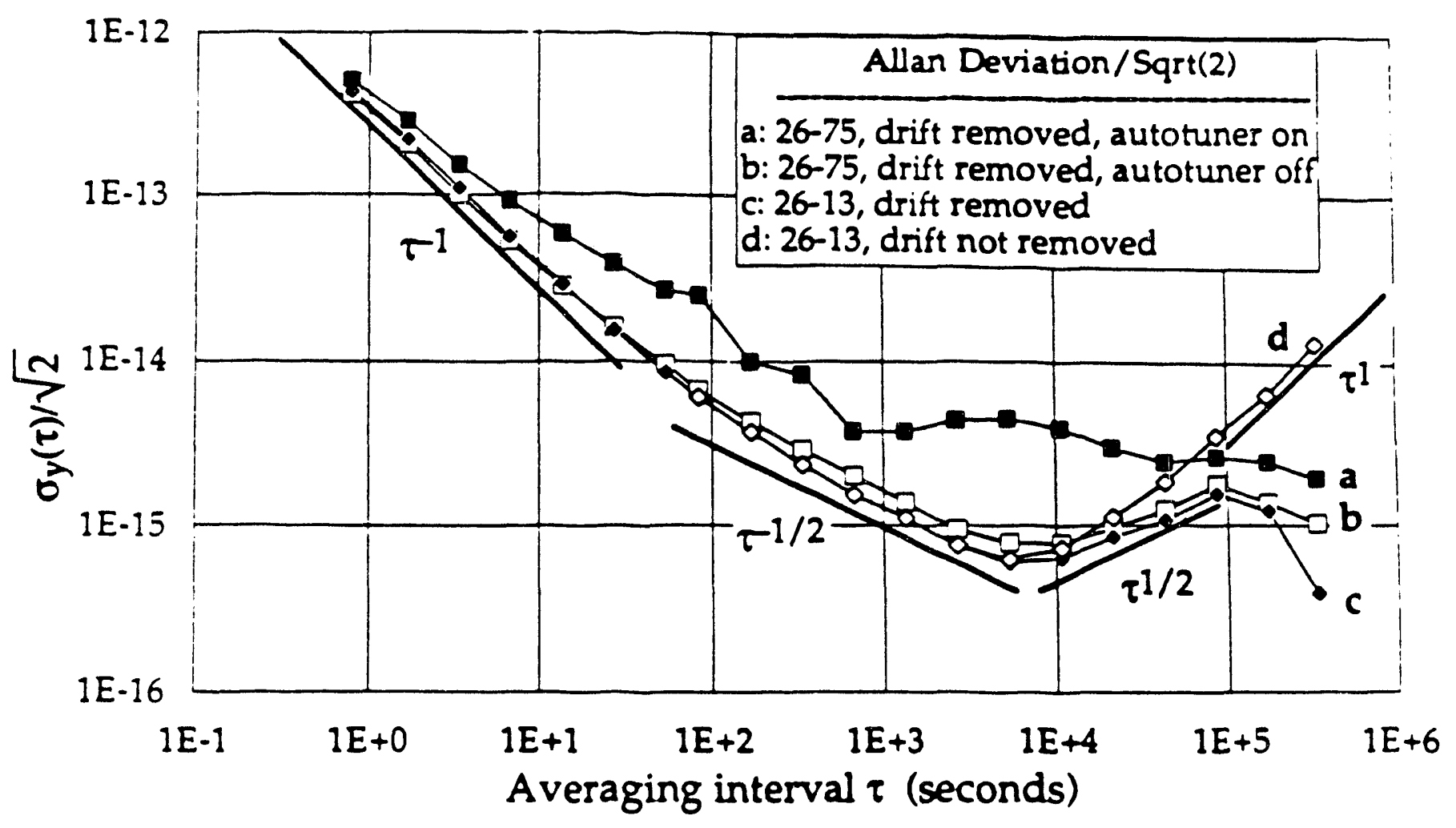

Figure 2

Frequencies Stabilities of Masers at SAO 


\section{APPENDIX A2}

FREQUENCY STANDARDS DESIGNED FOR SPACECRAFT USE AT THE LENINGRAD RADIOTECHNICAL SCIENTIFIC INSTITUTE 
(blank)

A -8 


\section{GEM}

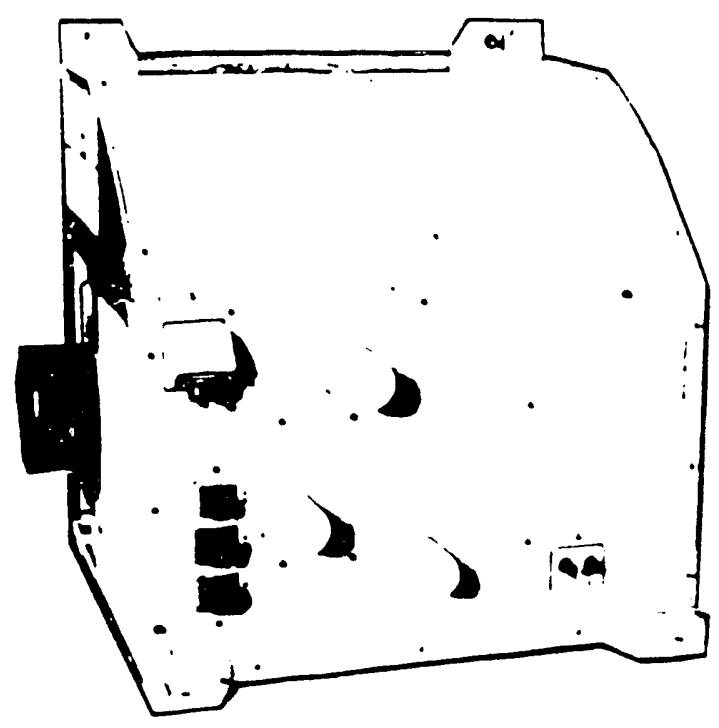

Cesium beam frequency standard for onboard space equipment

\section{Specifications}

- Output frequency

- Relative frequency accuracy over lifetime

- Stability, averaging time

- 1 second

- 100 seconds

- 1 hour

- 1 day

- Temperature change of frequency, $1 /{ }^{\circ} \mathrm{C}$

- Temperature range

- Power supply

- Power consumption

- Dimensions

- Weight

- Operational life
$5 \mathrm{MHz}$

$\pm 1.10^{-11}$

$5.10^{-11}$

$1.10^{-11}$

$2,5.10^{-12}$

$5.10^{-13}$

$5.10^{-13 \circ} \mathrm{C}$

$0 . . .+40^{\circ} \mathrm{C}$

27 VDC

Not greater than $80 \mathrm{~W}$ $370 \times 450 \times 500 \mathrm{~mm}$ $39.6 \mathrm{~kg}$

17,500 hours 


\section{MALACHITE}

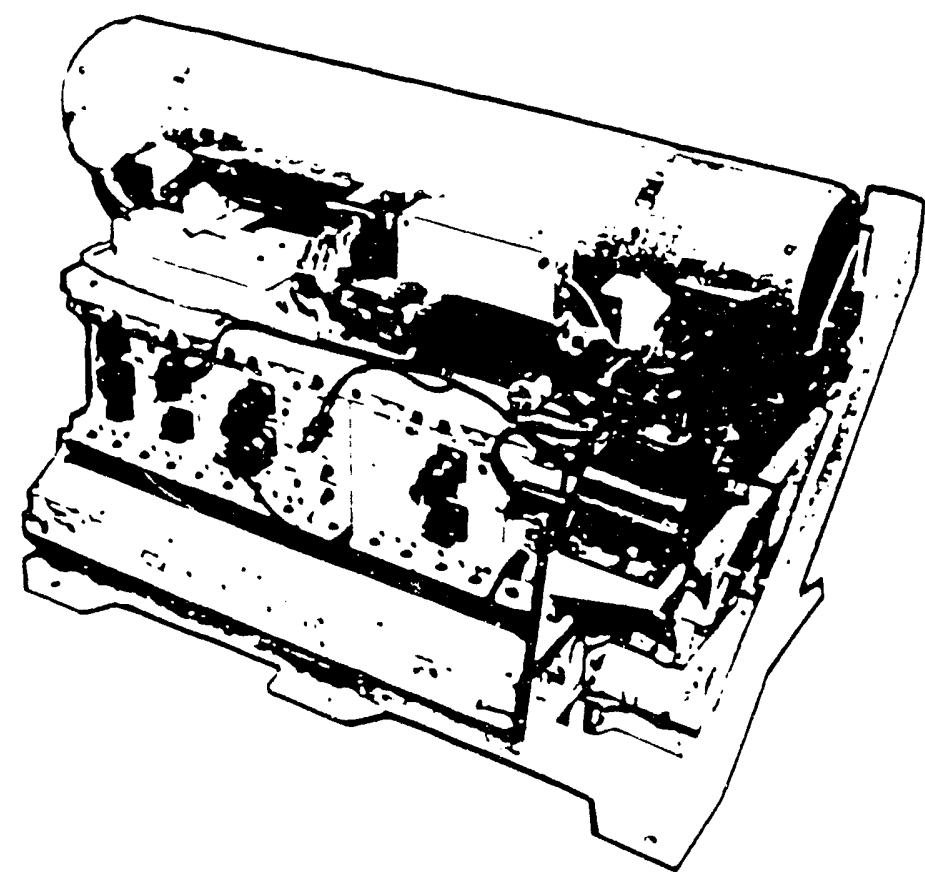

Cesium beam frequency standard for onboard space equipment

Specifications

- Output frequency

- Relative frequency accuracy over lifetime

- Stability, averaging time

- 1 second

- 100 seconds

- 1 hour

- 1 day

- Temperature change of frequency, $1 /{ }^{\circ} \mathrm{C}$

- Temperature range

- Power supply

- Power consumption

- Dimensions

- Weight

- Operational life
$5 \mathrm{MHz}$

$\pm 1.10^{-11}$

$2.10^{-11}$

$5.10^{-12}$

$5.10^{-13}$

$1.10^{-13}$

$2.10^{-13{ }^{\circ} \mathrm{C}}$

$0 \ldots+40^{\circ} \mathrm{C}$

27 VDC

$90 \mathrm{~W}$

$421 \times 414 \times 655 \mathrm{~mm}$

$52 \mathrm{~kg}$

28,500 hours 


\section{REFERENCE}

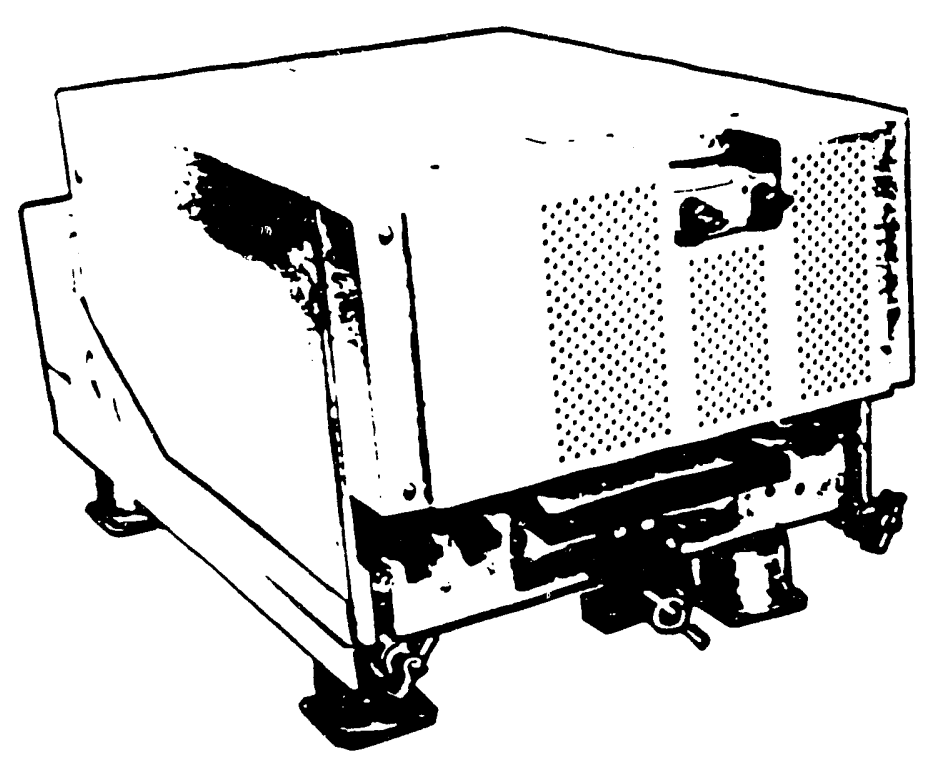

Rubidium cell frequency standard for airspace vehicles

\section{Specifications}

- Output frequency

- Relative frequency accuracy over lifetime

$5 \mathrm{MHz}$

- Stability, averaging time

- 1 to 100 seconds

$\pm 2,5.10^{-11}$

- 100 to 1000 seconds

- Temperature change of frequency, $1 /{ }^{\circ} \mathrm{C}$

- Frequency drift over 1 month of continuous operation

$(1-2) \cdot 10^{-11}$

$(2-5) \cdot 10^{-12}$

$2,0.10^{-12 \circ} \mathrm{C}$

- Temperature range

- Power supply

- Power consumption

- Dimensions (w/o mounting frame)

- Weight (w/o mounting frame)

$5.10^{-12}$

$-30 \ldots+55^{\circ} \mathrm{C}$

27 VDC

$35 \mathrm{~W}$

$257 \times 194 \times 320 \mathrm{~mm}$

$12.5 \mathrm{~kg}$ 


\section{AMETHYST}

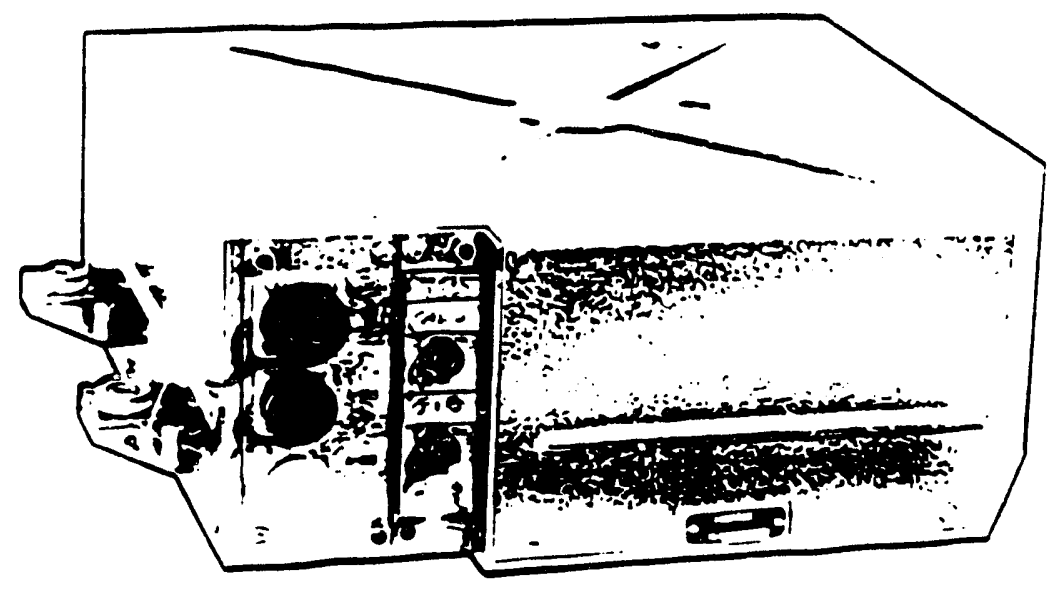

Rubidium cell frequency standard for space vehicles

\section{Specifications}

- Output frequency

- Relative frequency accuracy over lifetime

- Stability, averaging time

- 1 to 10 seconds

- Reproducibility from turn-on to turn-off

- Warm-up

- Temperature range

- Operational life in "quartz oscillator" mode

- Power supply

- Power consumption

- Dimensions

- Weight
$5 \mathrm{MHz}$

$\pm 3.10^{-10}$

$5.10^{-11}$

$5.10^{-11}$

2.5 hours

$-5 \ldots+40^{\circ} \mathrm{C}$

15,000 hours

27 VDC

$28 \mathrm{~W}$

$300 \times 280 \times 110 \mathrm{~mm}$

$8 \mathrm{~kg}$ 


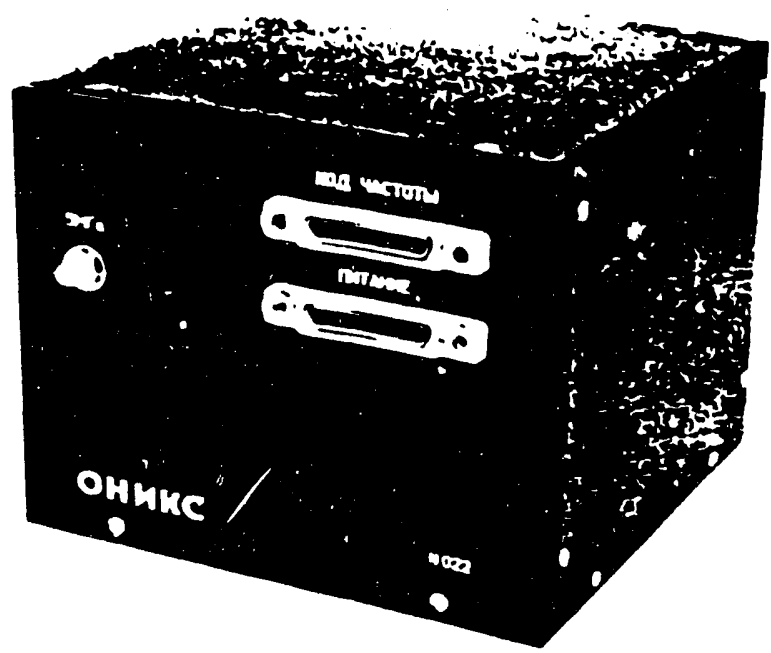

Rubidium cell frequency standard for airspace vehicles and ground equipment

\section{Specifications}

- Output frequency

- Relative frequency accuracy over lifetime

- Stability, averaging time

- 1 to 10 seconds

- Instrument error of frequency adjustment

- Frequency drift over 1 year of continuous operation

- Temperature range

- Operational life

- Power supply

- Power consumption

- Dimensions

- Weight
$5 \mathrm{MHz}$

2, $5.10^{-11}$

$5.10^{-11} \cdot \tau^{-1 / 2}$

$1.10^{-11}$

$3,5.10^{-10}$

$-60 \ldots+60^{\circ} \mathrm{C}$

50,000 hours

27 VDC

$20 \mathrm{~W}$

$130 \times 130 \times 110 \mathrm{~mm}$

$2 \mathrm{~kg}$ 


\section{BERYL}

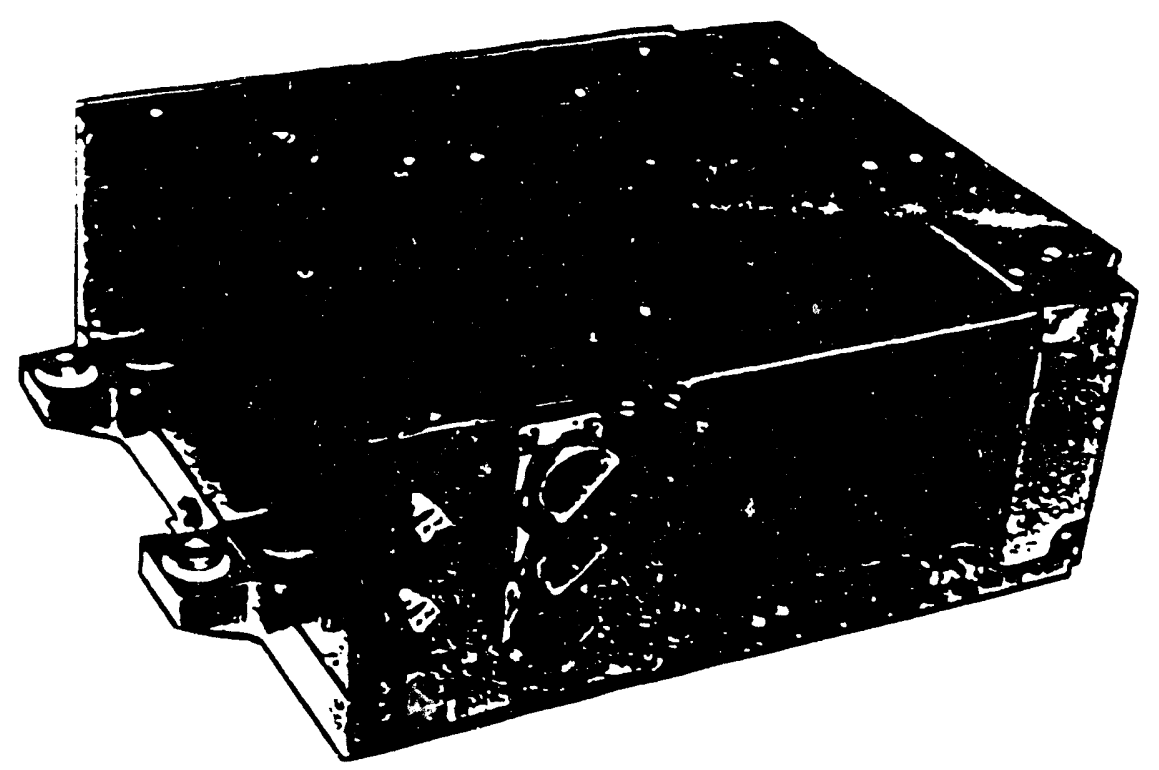

Rubidium cell frequency standard for space vehicles

\section{Specifications}

- Output frequency

- Frequency adjustment step

- Relative frequency accuracy over lifetime

- Stability, averaging time

$$
\text { - } 1 \text { to } 100 \text { seconds }
$$

- Frequency drift

- Temperature range

- Non-harmonic distortion in $100 \mathrm{kHz}$ bandwidth (5 MHz)

- Warm-up from $25^{\circ} \mathrm{C}$ to $\pm 5.10^{-11}$

- Power supply

- Power consumption in warm-up ( $30 \mathrm{~min}$.) in operation mode at $25^{\circ} \mathrm{C}$

- Dimensions

- Weight
$5 \mathrm{MHz}$

$2,2.10^{-11}$

$(2-3) \cdot 10^{-11}$

$2.10^{-11} \cdot \tau^{-1 / 2} /$ month

Not greater than $1.10^{-11} / \mathrm{mo}$.

$-5 \ldots+45^{\circ} \mathrm{C}$

Not less than $95 \mathrm{~dB}$ $30 \mathrm{~min}$.

23-24 VDC

$20 \mathrm{~W}$

$300 \times 268 \times 120 \mathrm{~mm}$

Not greater than $8 \mathrm{~kg}$ 


\section{LAZURITE}

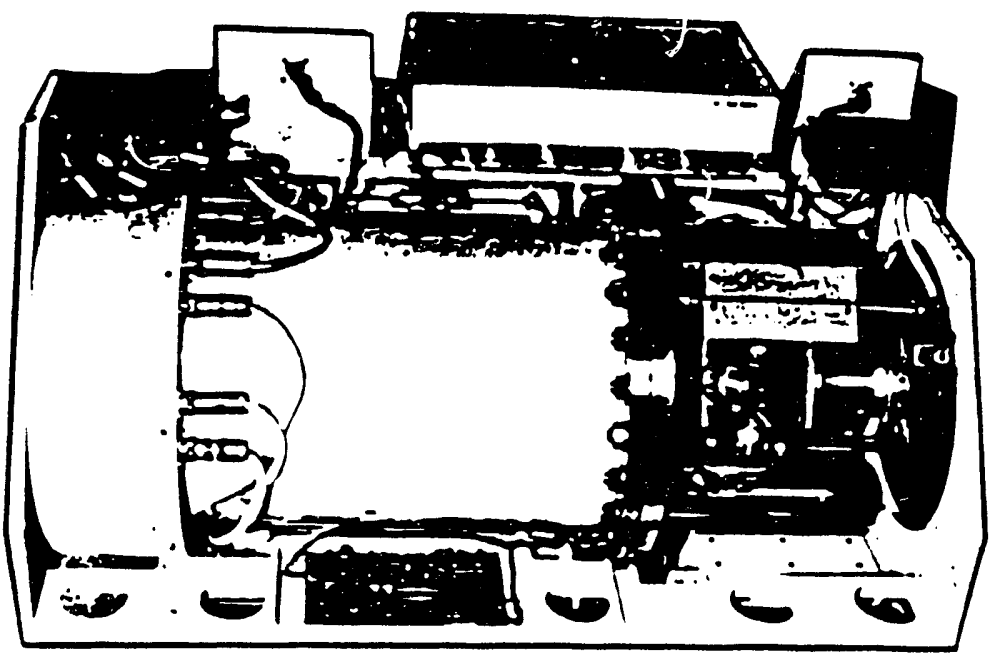

Compact hydrogen frequency standard for space vehicles

\section{Specifications}

- Output frequency

- Stability, averaging time

- 1 to 100 seconds

- 1 day

- Settability

- Operational life

- Power supply

- Power consumption

- Temperature range

- Volume

- Weight
$5 \mathrm{MHz}$

$1,5.10^{-12} \cdot \tau^{-1 / 2}$

$2.10^{-14}$

$1.10^{-14}$

15,000 hours

2:- VDC

$90 \mathrm{~W}$

$0 \ldots+40^{\circ} \mathrm{C}$

$75 \mathrm{dm}^{3}$

$40 \mathrm{~kg}$ 
(blank)

A-16 
APPENDIX A3

HYDROGEN MASERS FROM

GOR'KIY "QUARTZ" SCIENTIFIC PRODUCTION ASSOCIATION (NPO) 
(blank)

A-18 


\section{Active Hydrogen Maser}

Frequency and Time Standard

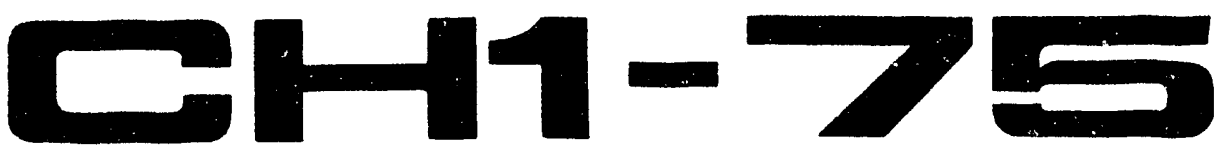

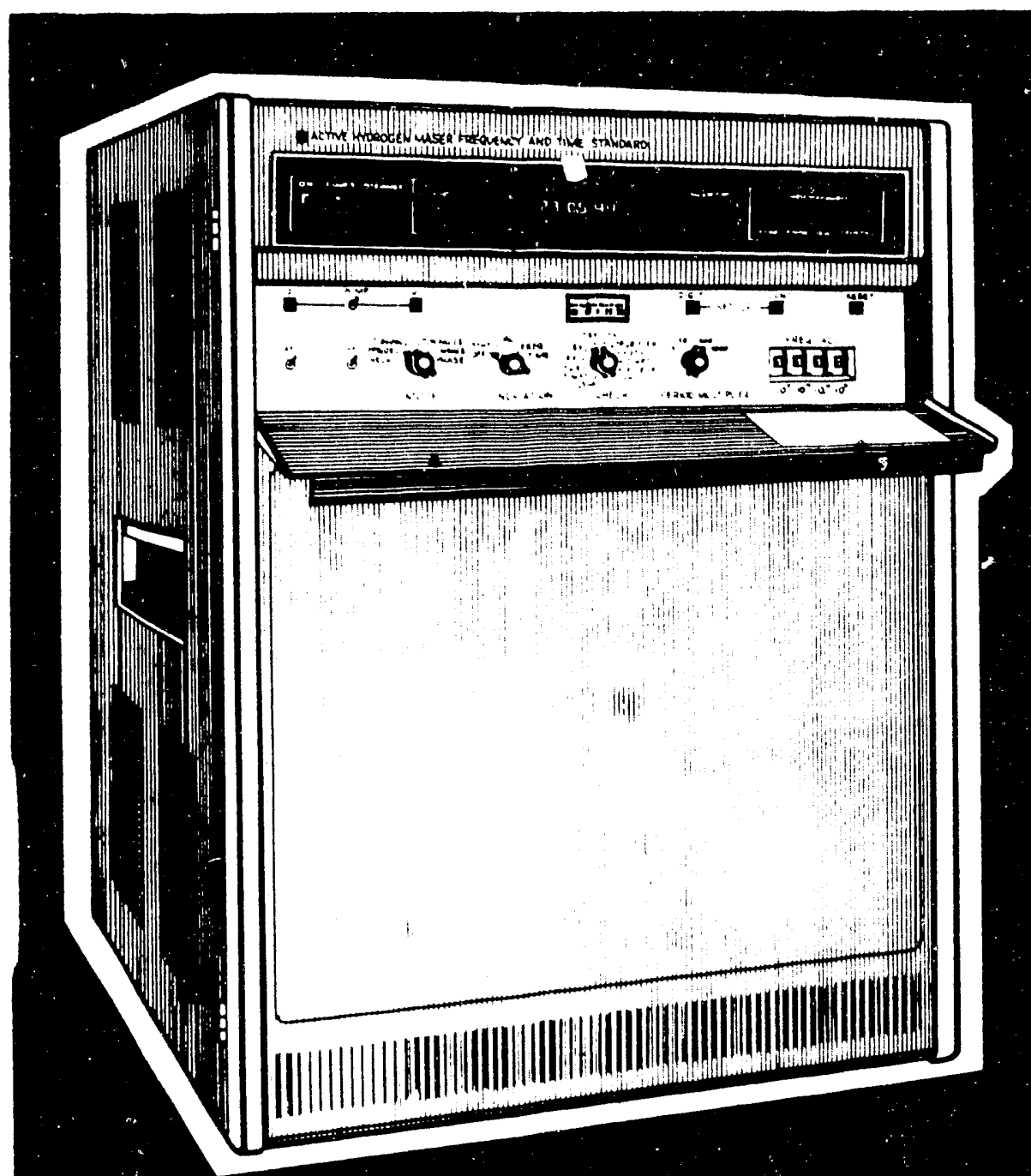

OUTPUT SIGNAL FREQUENCY STABILITY:

NOT MORE THAN $4 \cdot 10^{-13} / \mathrm{s}$ NOT MORE THAN $3 \cdot 10^{-15} / \mathrm{h}$ NOT MORE THAN $5 \cdot 10^{-15} / 24 \mathrm{~h}$ A-19 flll радиоизмерительная

BASED ON HYDROGEN MASER

\section{APPLICATION:}

NATIONAL TIME AND FREQUENCY CALIBRATION SERVICES

RADIOASTRONOMY

NAVIGATION

GEODESY

RFSF $\triangle R \cap H$ 


\section{Active Hydrogen Maser Frequency and Time Standard ㅁ-1-7E}

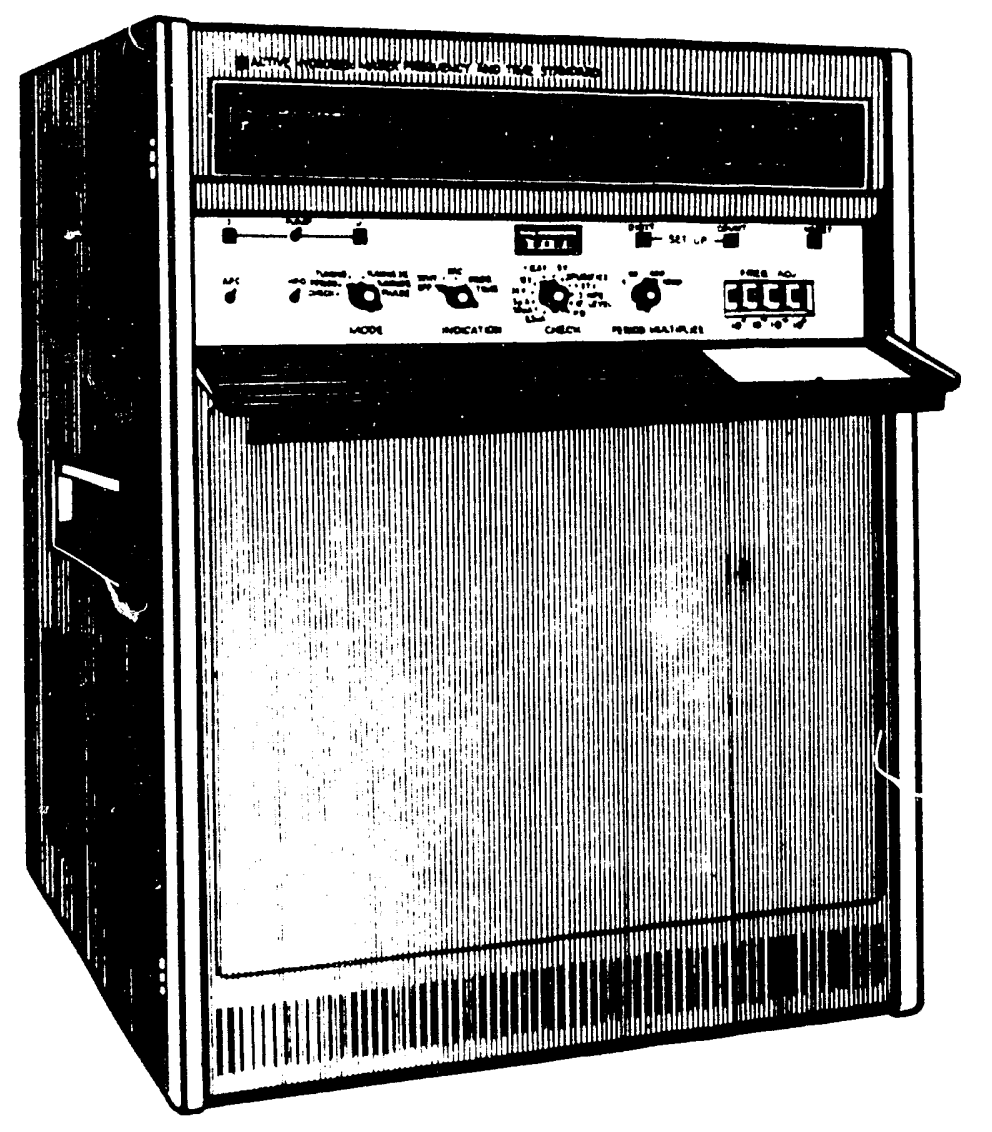

DESIGNED TO BE USED AS A SOURCE OF HIGHSTABLE PRECISE PURE SPECTRAL $5 \mathrm{MHz}$ AND $100 \mathrm{MHz}$ SIGNALS FOR TIME AND FREQUENCY KEEPING 


\section{COMPOSITION AND PRINCIPLE DF OPERATION}

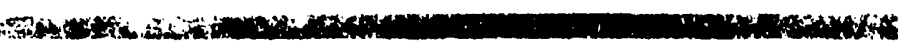

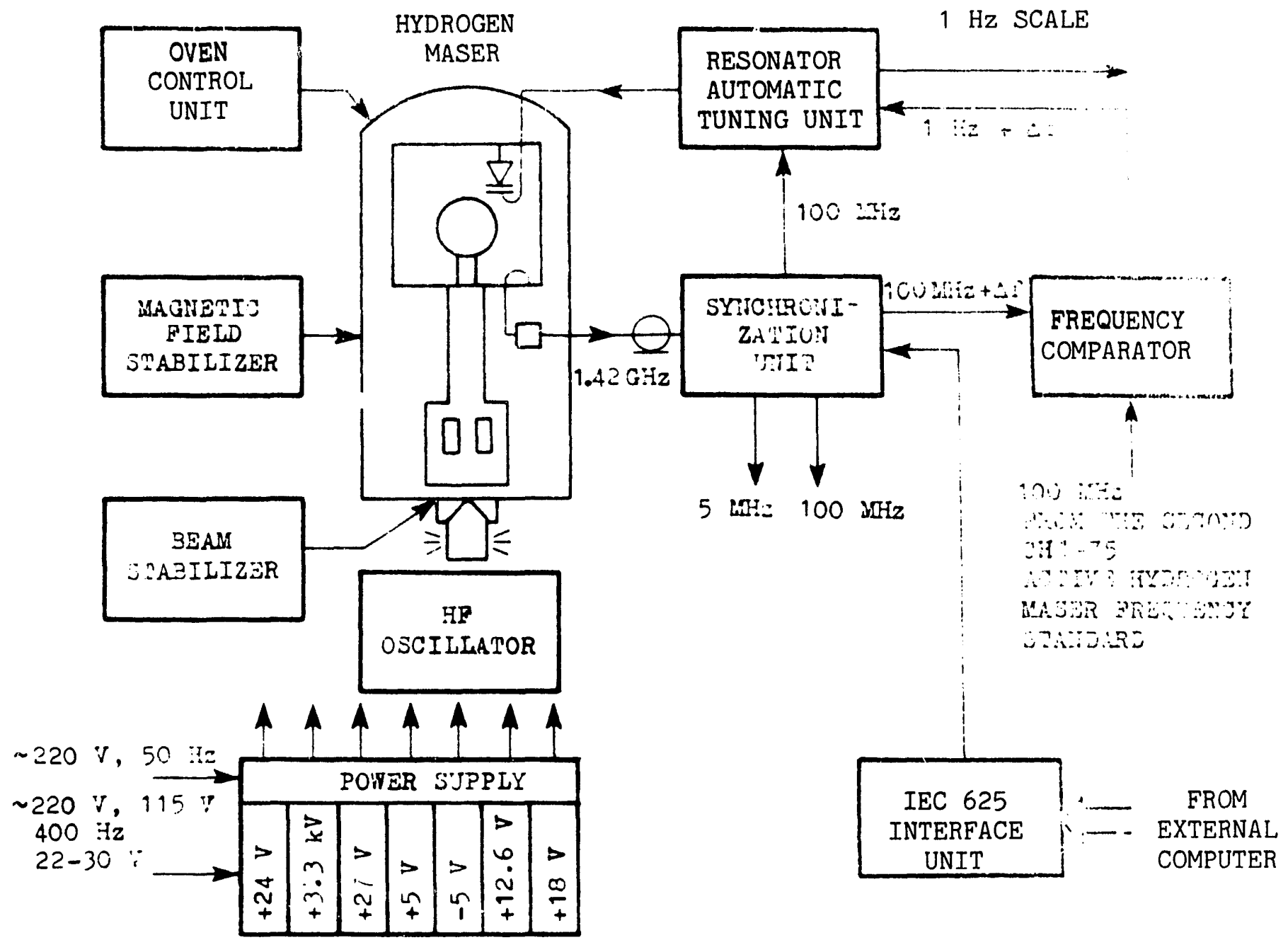

P18.1. CH1-75 Simplifled Block-d1aOram.

The CH1-75 principle of operation

(P1g.1) 1s besed on 5 lite cryotal oscillator Prequency phase locking to the hydrogen meser sigmal. 


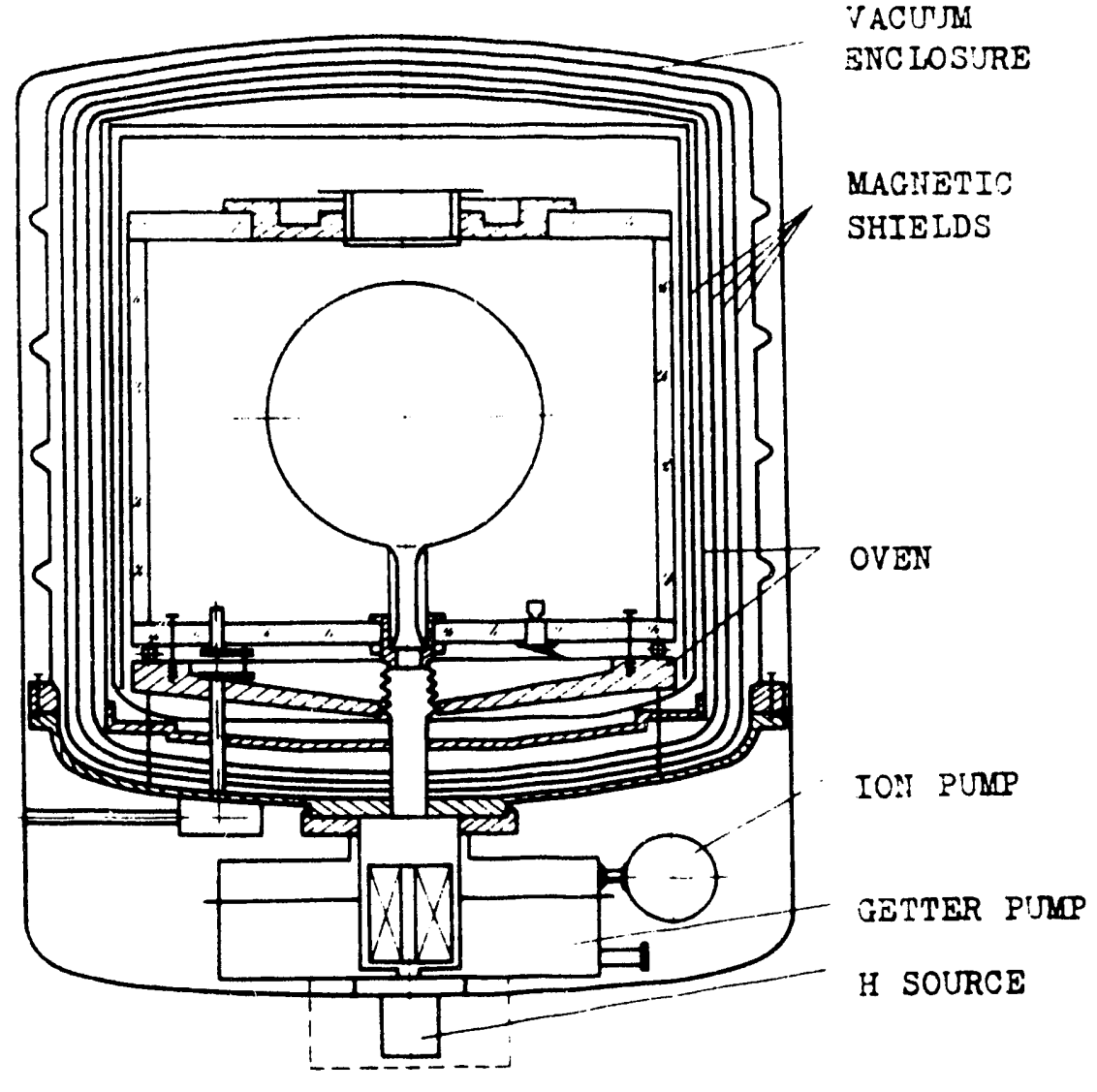

Pig.2. Hydrogen maser schematic

Metrological characteristics are provided by a hydrogen maser (Pig.2), having the following featurea:

-unique storage bulb plating, made of Pluorinated copolymer, providing high $\theta$ of emlasion line $\left(Q_{1}>2 \cdot 10^{9}\right)$ at bulb volume 21 , low irequency offset $(2-5)$. $10^{-12}$ due to hydrogen atom interaction with storage bulb plating, high reproducibility and ilwe stability;

glass ceramic resonator and iwo-stage multizone oven, enclosed in a low vacuum chamber, provide temperature coefficient of frequency $(1-5) \cdot 10^{-15}$ in temperature range $5-40^{\circ} \mathrm{C}$. Power consumption of the oven 18 not more than $2 \mathrm{~W}$;

- resonator frequency retuning by mechanic and electronic neans allows to retune Prequency in the range $2 \cdot 10^{-10}$ and to tune it manually and automatically to ihe opectral line top erequency in steps $(1-2) \cdot 10^{-15}$;

- magnetic shield system, consisting of fivelayer permalloy 81 HMA shield and magnetic field stabilyzer, provides dynamic shielding factor $\sim 100 \mathrm{~dB}$;

- hydrogen atom unpolarized bearn, produced by atom mixing at $\mathrm{P}=1, \mathrm{~m}_{\mathrm{F}}= \pm 1$ levels, reduces signiflcantly nonuniform fleld effect on oscillation frequency stability in microwave resonator area;

three-chamber vacuum system, evacuated by getter and ion pumpe of original construction, providea vacuum below $1 \cdot 10^{-8}$ $\mathrm{mm} \mathrm{Hg}$ in the storage bulb and in resonator cavity;

power consumption 25-30 W;

- weight $45 \mathrm{~kg}$. 


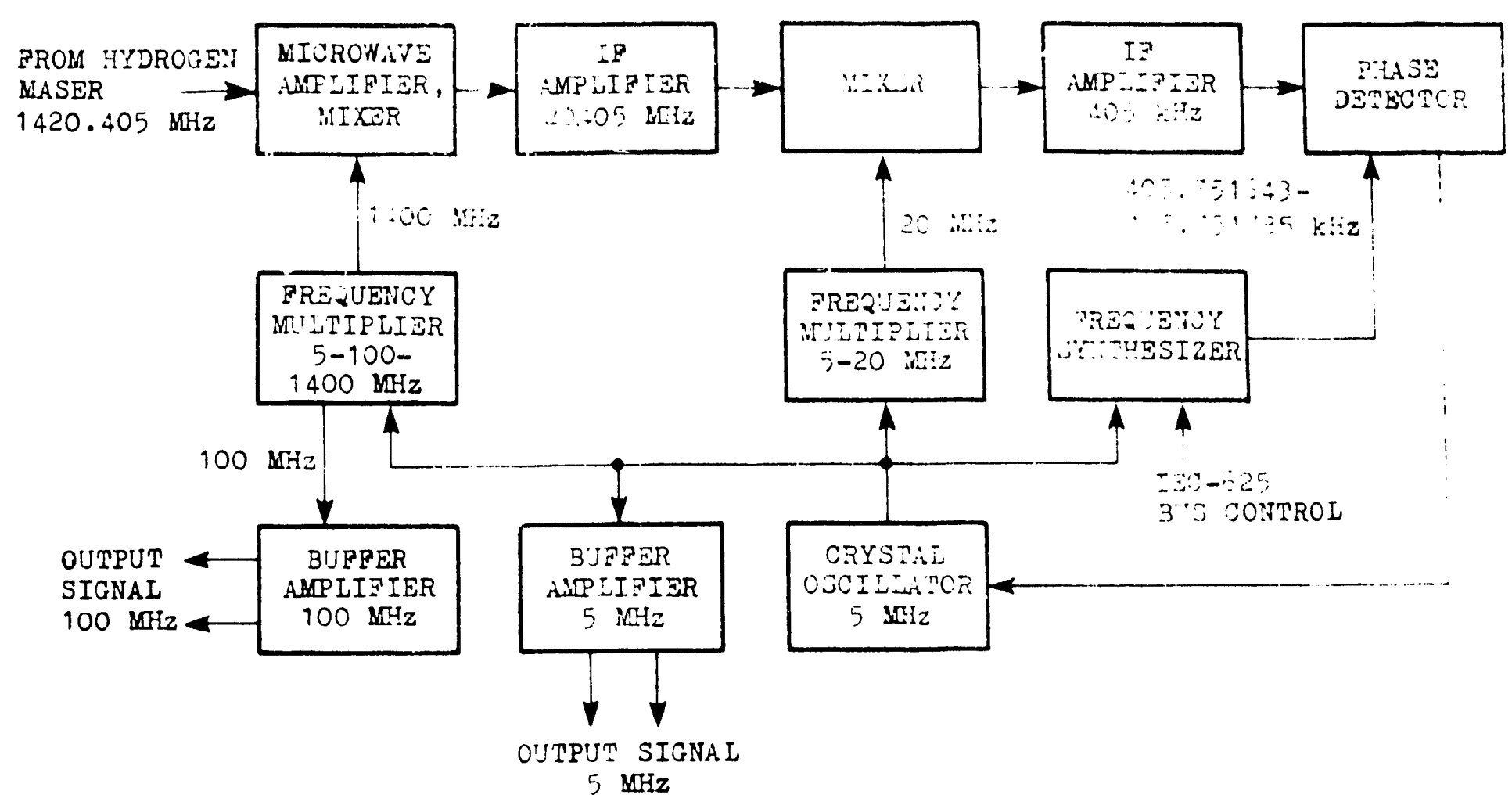

Fig.3. Synchronization unit block diagram

Synchronization unit amplifies $1 \cdot 10^{-13} \mathrm{i}$ hydrogen maser signal by the help of superheterodyne receiver, synchronizes $5 \mathrm{MHz}$ crystal oscillator signal phase to hydrogen maser signal phase and shapes $5 \mathrm{MHz}$ and $100 \mathrm{kHz}$ spectral-pure sine-wave standard slgnals (Fig.3).

\section{Features:}

- hydrogen maeer signal is amplified by RP transistor amplifier, having low nolse factor ( $2 \mathrm{~dB})$;

- interference nolse suppression in 1mage channel;

- large multiplication factor of first irequercy multiplier stages provides high iempera:ure stablilty (temperature coefflcient of phase is less than $\left.0.01 \mathrm{~ns} /{ }^{\circ} \mathrm{C}\right)$;
- sample and hold phase detector with proportionally integrating operational amplifier provides PLL optional noise band $3-5 \mathrm{~Hz}$.

\section{FREQUENCY COMPARATOR}

Designed for increasing hydrogen frequency standard phase difference measurement resolution using resonator automatic tuning system. Allows to measure $100 \mathrm{MHz}$ external algnal frequency otability. Features:

- RP $99 \mathbf{M H z}$ crystal oscillator used as a local oscillator;

- local oscillator PLI synchronized to input algnal frequency. 
$100 \mathrm{MHz}+\Delta f$

SIGNAL PROM

THE TUNED

FREQUENCY

STMIDPRD

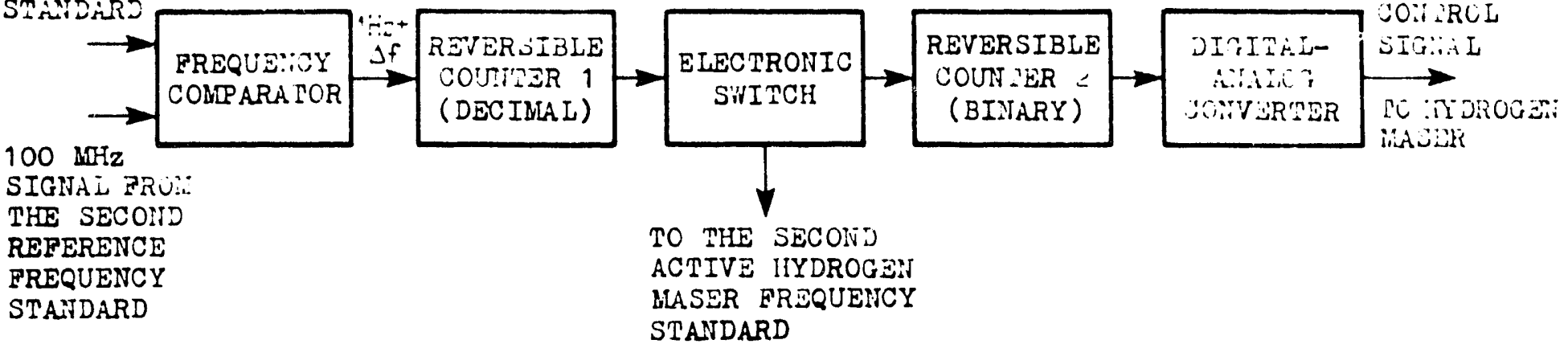

\author{
Pig. 4. Hydrogen oscillator resonator automatic frequency \\ tuning b lock-diagram
}

Designed for hydrogen maser resonator automatic frequency tuning to the spectral line top frequency, time scale pulse shaping with repetition rate 18 ; provides current time indication in hours, minutes and seconds, fault indication is hydrogen maser assemblies, in synchronization unit and performs gelfdiagnostics.

Resonator automatic frequency tuning to the bydrogen atom emlsaion spectral line top frequency (Pig.4) is performed in the following manner:

$100 \mathrm{MHz}$ sienal from the multiplier output 5-100 MHz, located in aynchronization unit, is applied to the frequency comparator input, the second input of which recelves $100 \mathrm{MHz}$ olgnal from the eecond hydrogen maser.

Prequency comparator converts $100 \mathrm{MHz}$ olgnala into $1 \mathrm{~Hz}$ signal proserving phase ifference $\Delta \rho$. Resonator tuning provides Q-modulation of hydrogen maser spectral line. Reversible counter 1 counts beat frequency of two algnals during 100 seconds in forward direction at one spectral line $Q$, and during 100 seconde in backward direction at another $Q$. Rever- sible counter result (the result difference of counting in forward and backward directions) is proportional to hydrogen maser resonator frequency tuned away from apectral line top erequency. Resonator tuning is performed sceording only to detuning sign data, that 18 applied to reversible counter 2 input and converted into voltage by a digitalanalog converter. DAC output roliage 18 applied to hydrogen maser resonator varicap, for correcting its erequency. Adjustment is made in cycles of 250 a duration. Frequency comparator and counter 1 control frequency stablilty of $100 \mathrm{MHz}$ external signal sources; the results are indicated on a digital display. Counter resolution is in the range $1 \cdot 10^{-13}-1 \cdot 10^{-16}$ at measurement period $1-1000$ 8.

\section{IEC. 625 INTERFACE}

Designed for standard frequency control and monitoring via ar external computer and also for trangferring diagnostic assembly state data. 


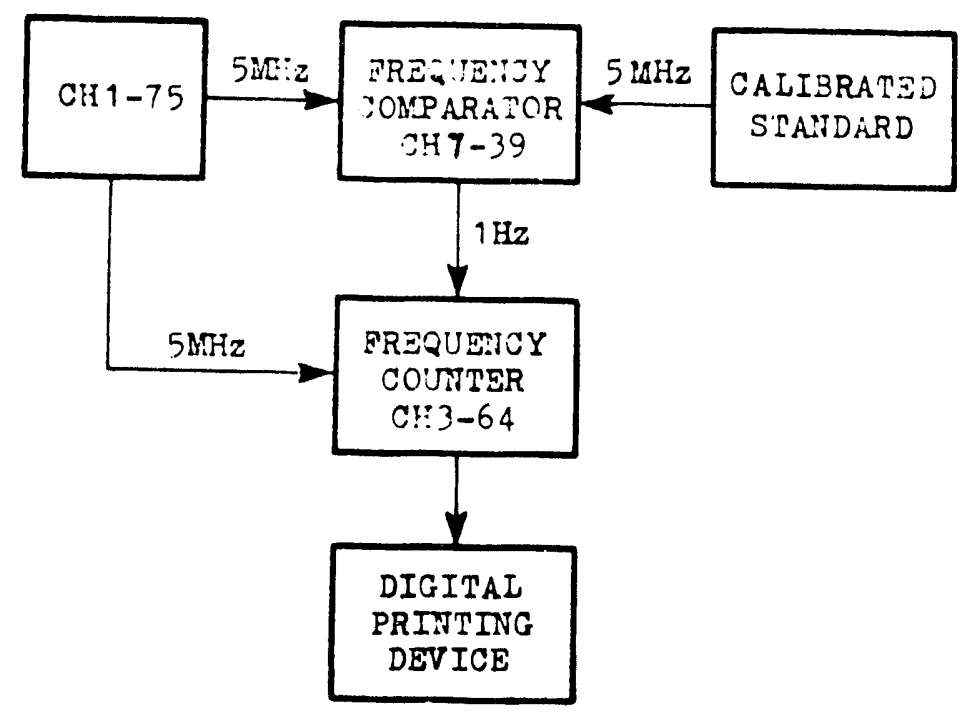

Pig.5. Frequency standard calibration block-diagram using external frequency comparator

Active maser frequency standards provide metrological verification of quantun frequency standards of different types (rubidiut, cesium, hydrogen and others) during their development, production and service. (Pig.5,6).

- On the base of time and frequency hydrogen standards small-size time and frequency systems are developed.

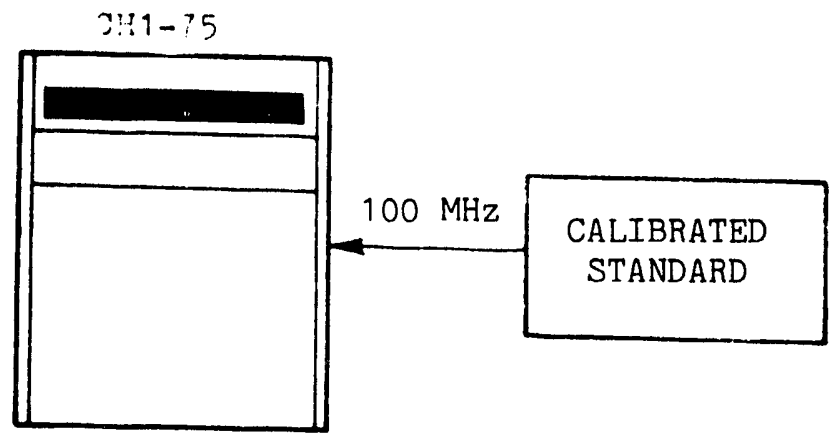

F1g.6. Prequency standard calibration. block-diagram using a buili-in comparator and a Prequency counter

- High resolution satellite NAV syotems can be bullt on hydrogen frequency standards base.

- Radiolnterferometer with superlong base, using hydrogen frequency standarda, provides angular resolution $\sim 10^{-4}$ seconds of arc, that for example allows to determine continent shift with centimetric error.

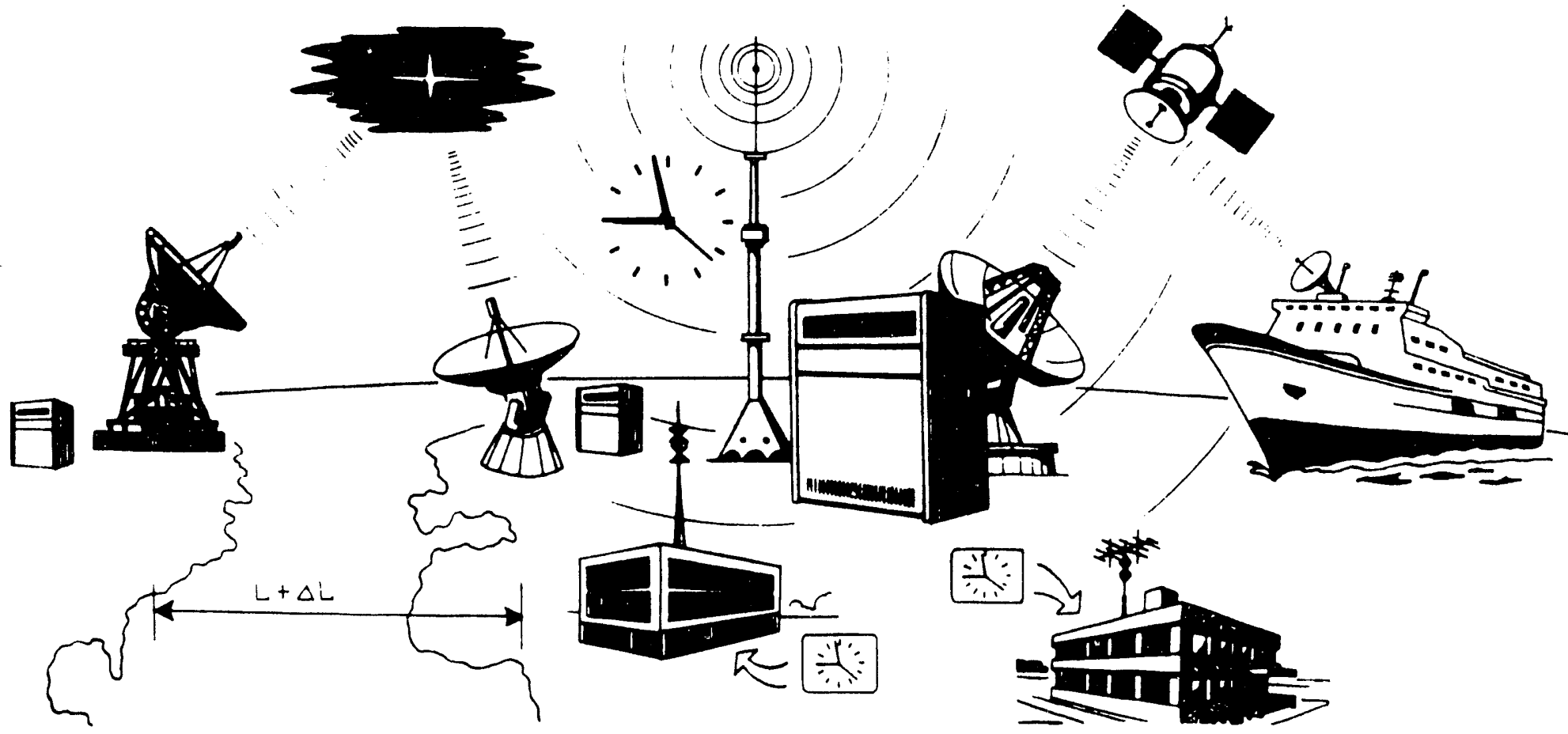




\section{SPECIFICATION}

Output freque::cy: $5 \mathrm{MHz}, 100 \mathrm{MHz}$.

Ou put siz:ia? vol;age RMS value $(1 \pm 0.2)$ v at load 50 ohm.

Prequercy relatire accuracy: not more than $\pm 1 \cdot 10^{-12}$ (during one year period).

5 and $100 \mathrm{MLiz}$ output algnal frequercy atab1lity (RuS relative two-gelective frequen-

cy deviation or Allan variance) not more than: $4 \cdot 10^{-13}$ ( $\left.1 \mathrm{~s}\right) ; \quad 3 \cdot 10^{-15}(1 \mathrm{~h})$;

$4 \cdot 10^{-14}(10 \mathrm{~s}) ; \quad 5 \cdot 10^{-15}(24 \mathrm{~h})$.

$8 \cdot 10^{-15}(1008)$;

Prequency atability per 100 8, 1 hour and 24 hours

is provided $a:{ }^{+}{ }^{\circ} \mathrm{C}$ ambient temperature change

in operatins emperature range $5-40^{\circ} \mathrm{C}$

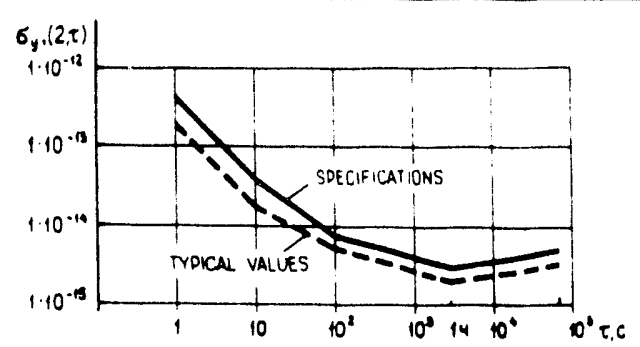

Prequency stability $5 \cdot 10^{-15} / 24 \mathrm{~h}$ is achived, when reference gignal io applied from the second CH1-75 active hydrogen maser frequency atandard.

Additional frequency variation at $1^{\circ} \mathrm{C}$ amblent temperature change: not more than $5 \cdot 10^{-15}$. Frequency reproducibility relative error: not more than $5 \cdot 10^{-14}$.

Frequency compensator resolution $1 \cdot 10^{-14}$ in retuning range is not less than $1 \cdot 10^{-10}$.

SSB noise power spectral density 18 not more than: - $125 \mathrm{~dB} / \mathrm{Hz}(20 \mathrm{~Hz})$;

- $130 \mathrm{~dB} / \mathrm{Hz}(330 \mathrm{~Hz})$;

$-135 \mathrm{~dB} / \mathrm{Hz}(1 \mathrm{kHz})$.

Prequency measurement error due to the bullt-in comparator is not more than:

$$
\begin{array}{ll}
1 \cdot 10^{-13} \text { (per 18); } & 3 \cdot 10^{-15} \text { (per } 100 \text { s); } \\
1,5 \cdot 10^{-14} \text { (per } 10 \text { g); } 5 \cdot 10^{-16} \text { (per } 1 \text { hour). }
\end{array}
$$

1 Hz pulse signal paraneters: amplitude - $2.5 \mathrm{~V}$ in 50 Ohm;

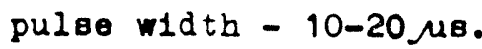

Pulae signal aynchronization 18 performed by $1 \mathrm{~Hz}$ positive polarity pulseg.

Syachronization error is not more than $\pm 50 \mathrm{as}$.

Power: $(220 \pm 22) \nabla,(50 \pm 0.5) \mathrm{Hz},(220 \pm 11) \mathrm{V},(115 \pm 5.75) \mathrm{V},(400 \pm 28) \mathrm{Hz}$.

In case of emergency the hydrogen standard switches automatically to DC external power supply 22-30 $\nabla$ (is not included in the kit).

Power conbumption: AC - $150 \mathrm{VA}$; DC - $100 \mathrm{~W}$.

Dimengions: $480 \times 550 \times 680 \mathrm{~mm}$.

We1ght: $90 \mathrm{~kg}$.

Operating cond1t1ons:

environmental temperature: $5-40^{\circ} \mathrm{C}$;

rela:ive bumidity: up to $80 \%$ at $25^{\circ} \mathrm{C}$;

atmospher1c preseure: $84-106 \mathrm{kPa}\left(630-795 \mathrm{mmHg}_{\mathrm{g}}\right)$.

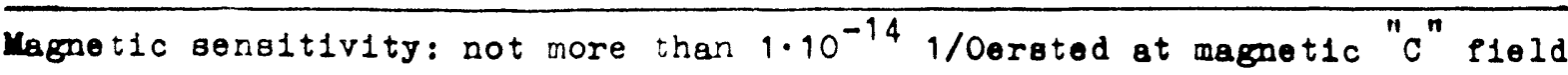
strength 0.5 moersted.

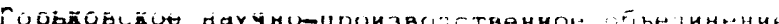

rero anano: .m. A-26

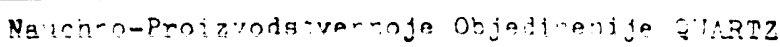

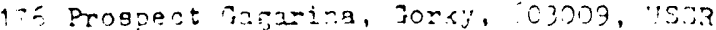




\section{Passive Hydrogen Maser Frequency Standard

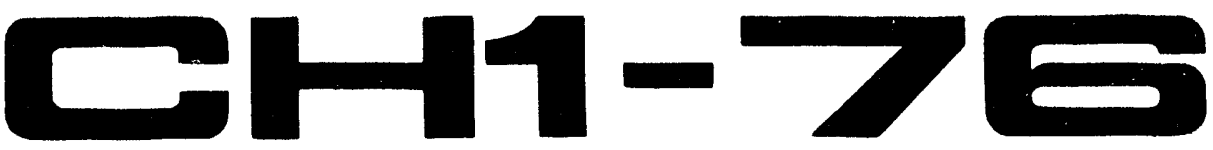

IIllf paаиоизмерительная

BASED ON A QUANTUM HYDROGEN

\section{DISCRIMINATOR}

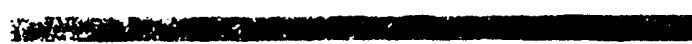

\section{APPLICATION:}

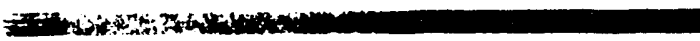

NATIONAL TIME AND

FREQUENCY CALIBRATION SERVICES

RADIOASTRONOMY

NAVIGATION

GEODESY

RESEARCH

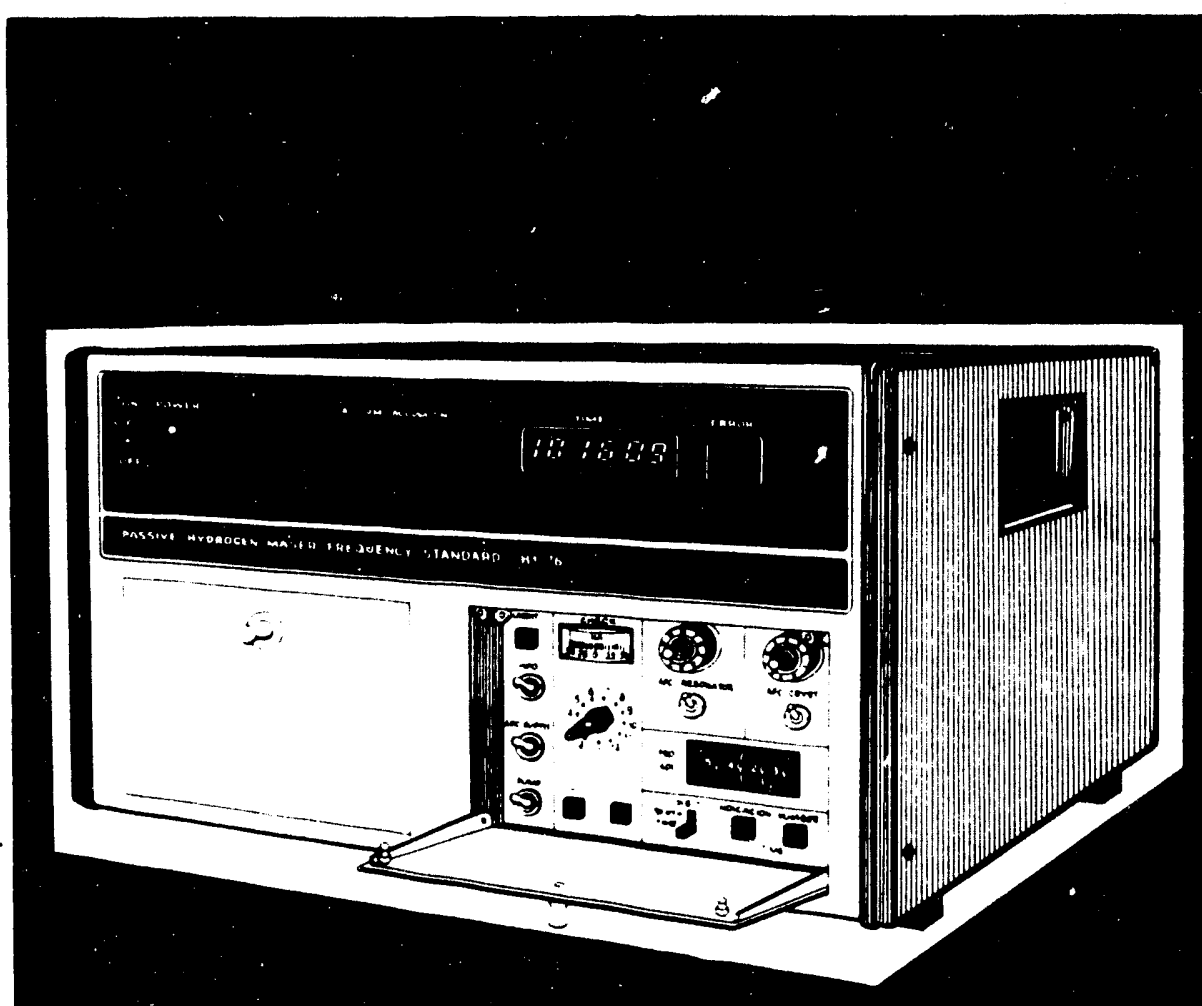

OUTPUT SIGNAL FREQUENCY STABILITY:

NOT MORE THAN $3 \cdot 10^{-12} / \mathrm{s}$

NOT MORE THAN $3 \cdot 10^{-14} / 24 \mathrm{~h}$ 


\section{Passive Hydrogen Maser Frequency Standard}
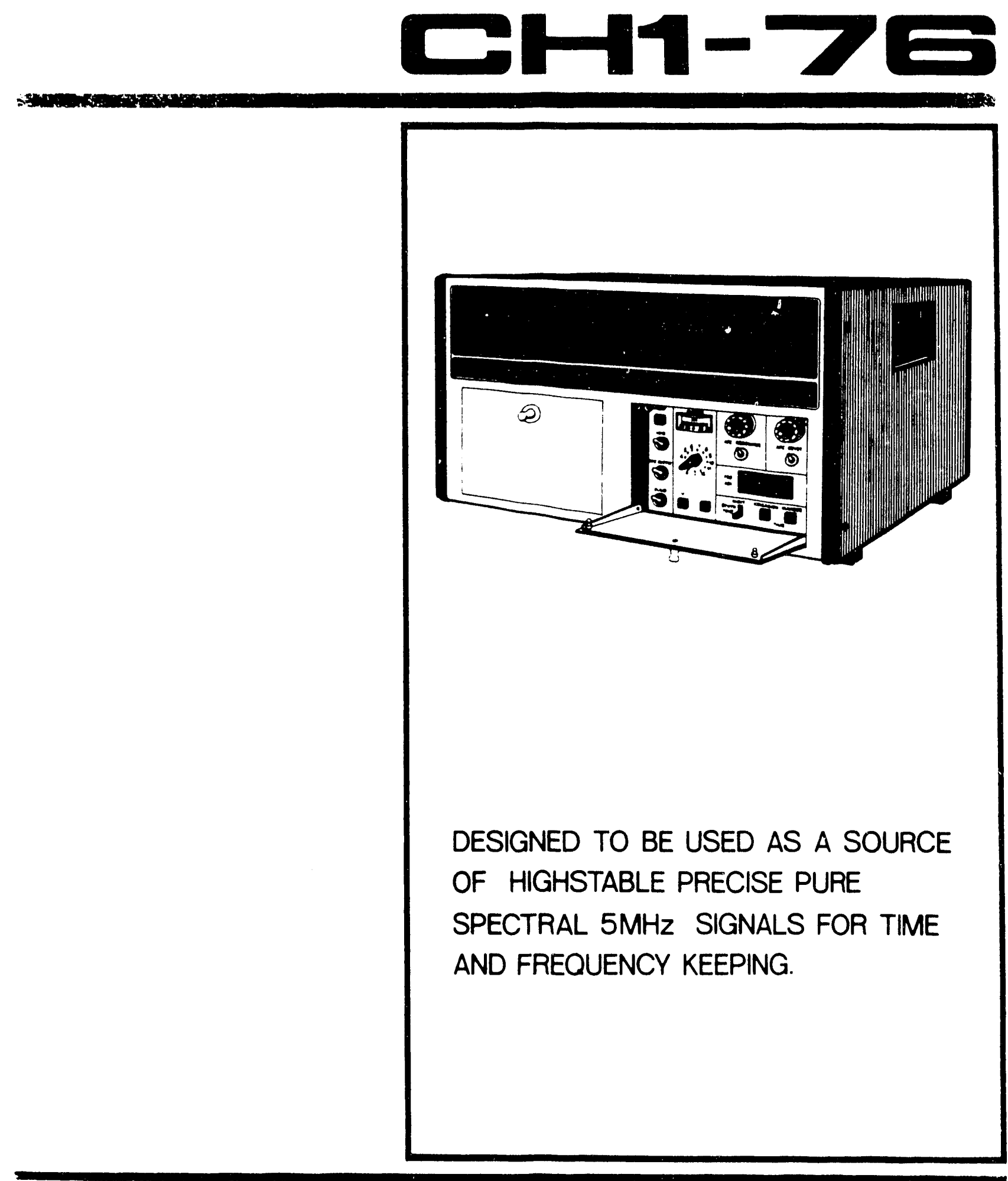
Te sel- principle o: operation is based on 5 iniz crystal sscillator aldtomaic erequency locking to the hydrogen atom e:ilssion line Prequency. Pasitve hydrogen irequency gtandard block-diagram 18 given or: 15.1 .

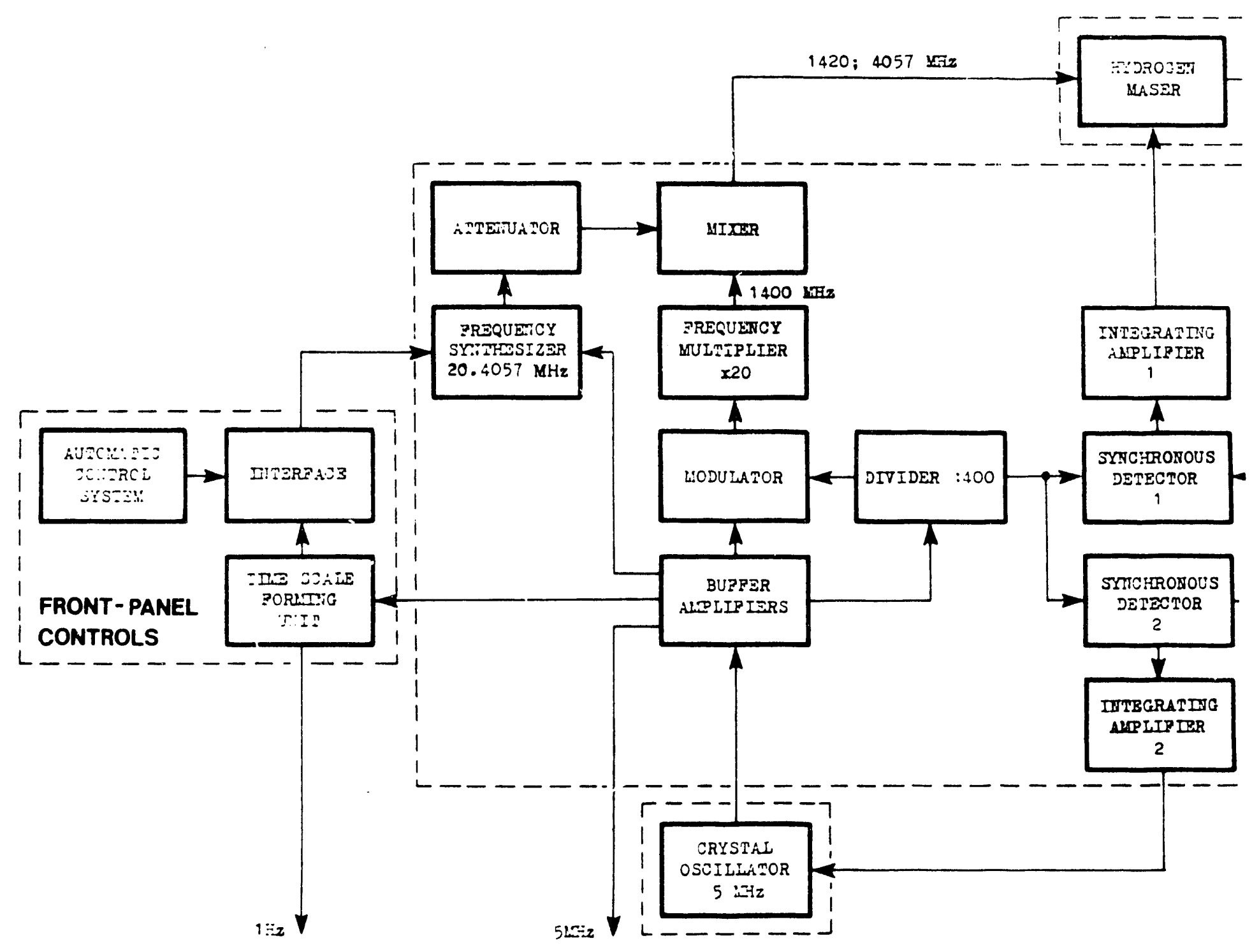

Fig. 1. Passive hydrogen frequency standard block diagram. 


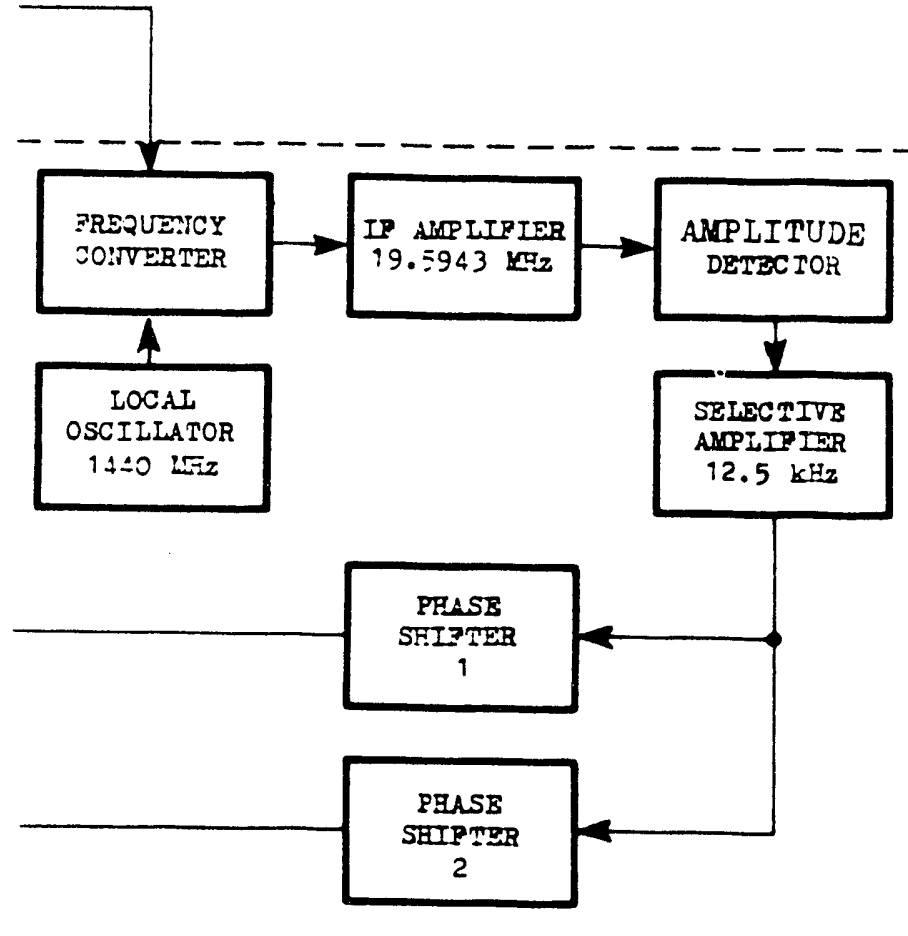

AFC UNIT

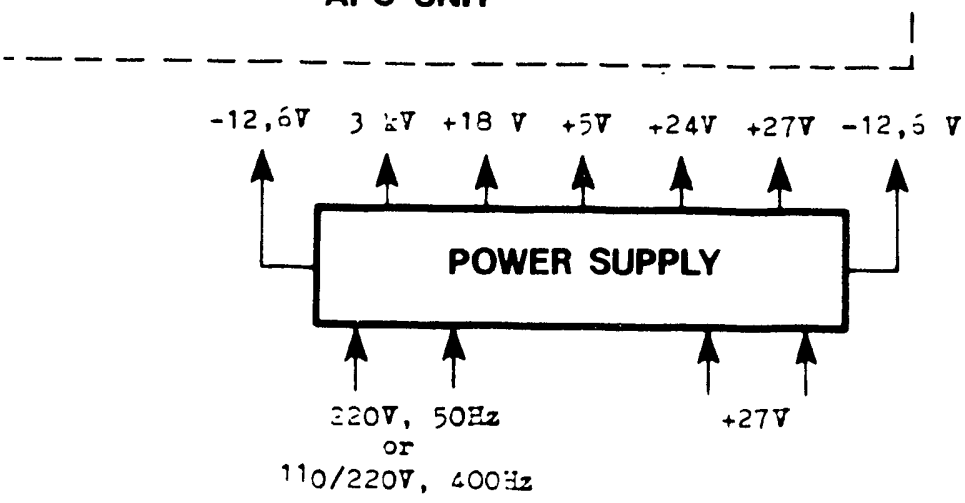

\section{AFC UNIT}

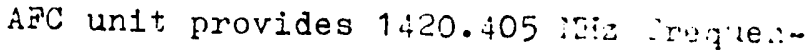
oy-modulated signal drivirs ine diccrialnator. In AFC unit signal is rodulated $i$. phase by $12.5 \mathrm{kHz}$ signal, i..ultiplied by 20, mixed with $20.4057 \mathrm{MHz}$ sisnal, genersved by the frequeacy urritiesizer, and $i$ applied to the muliplication diole, :hi is terminated by the quartur discril:ing or reso:lator.

Interacting with the atom emisaion line and discriminator resonator, frequency-modulated signal is converted into amplitudemodulaied signal. Signal envelope phase and amplitude show crystal oscillator trequency deviation from hydrogen atom smission line frequency and also discriminator resonator frequency deviation from crjaial oscillator frequency. From discrimirator output AM signal 18 applied back to the A'C unit, where it is amplified, converted and detected. From the amplitude detector $12.5 \mathrm{kHz}$ signal is applied to the select1ve amplifler, the output of which 1o applied to the phase shifters, separating resonetor and cryatal oscillator detuning algnals. Synchrodetectors and integrating amplifiers shape these signals that control resonator and crystal oscillator frequency.

\section{FRONT-PANEL CONTROLS}

Prov1de:

- output frequency manual control iroi the Pront panel and remote output irequency control through GPIB;

- pulse shaping time scale generation;

- current time Indication;

- Instrument troubleshooting and fault indication.

\section{POWER SUPPLY}

Applies required currents and voltages to the standard assemblies. 
QUANTUM HYDROGEN DISCRIMINATOR

The instrument metrological characteris:i $=s$ are provided by a quantum hydrogen discrimi:ator (F1g.2), having the rollowing features:

- storage bulb, plated by fluorined copolimer, provides $10^{5}$ hydrogen atom collisions againgt bulb walls without changlng their quantum state; emission line $Q$ factor is $\geqslant 1 \cdot 10^{9}$ at bulb volume $\sim 0.5 \mathrm{dm}^{3}$.

- RP metallic resonator of a original mechanical construction together with magnetic shields is placed under high vacuum $\leqslant 10^{-5} \mathrm{~mm} \mathrm{Hg}$, and is controlled by an oven. Oren power consumption at $50^{\circ} \mathrm{C}$ is not more than 1 in.

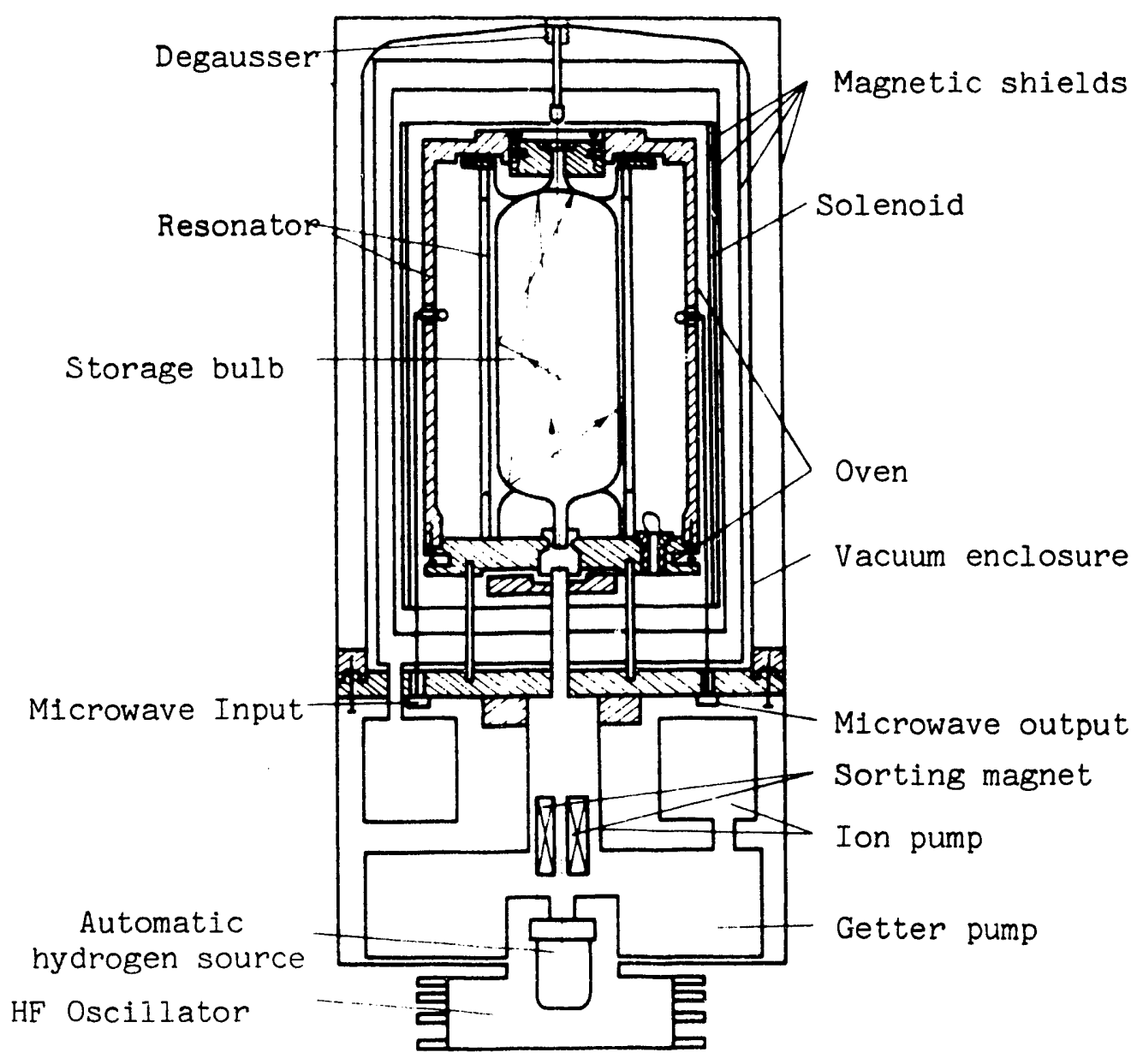

lig. 2. Quantum hydrogen diocriminator schematic arrangement (design). 
y Lia metic shields e: lectively remo:e

iagrezic field ef:ecss o enission line stability. Dynaric shielding "actor is; no: less than $50 \cdot 10^{3}$;

Corpact, ge:ter-purp, molecular iydroEe: source, atomic hydrogen ro.1rc made $0:$ super-pure crystal glass provide diricriminator continuous operation for a lons, period of tine (not less ihan 3 years).

\section{APPLICATION}

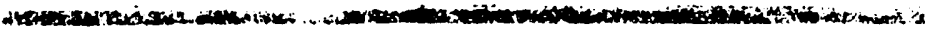

Navigation, radiolocation, metrology, ocientific researches, digital communication:

CH1-75 passive hydrogen maser can be ueed in high resolution navigation systems, such as NAVSTAR.

: Passive hydrogen meser provides $\mathrm{Rb}$ and Ce frequency standard verification at production line.

- CH1-76 passive hydrogen maser can serve as a base for national time and frequency syatems.

- Passive hydrogen maser - based radiointerferometer with a superlong base provides angle resolution $\sim 10^{-4}$ seconds of arc,

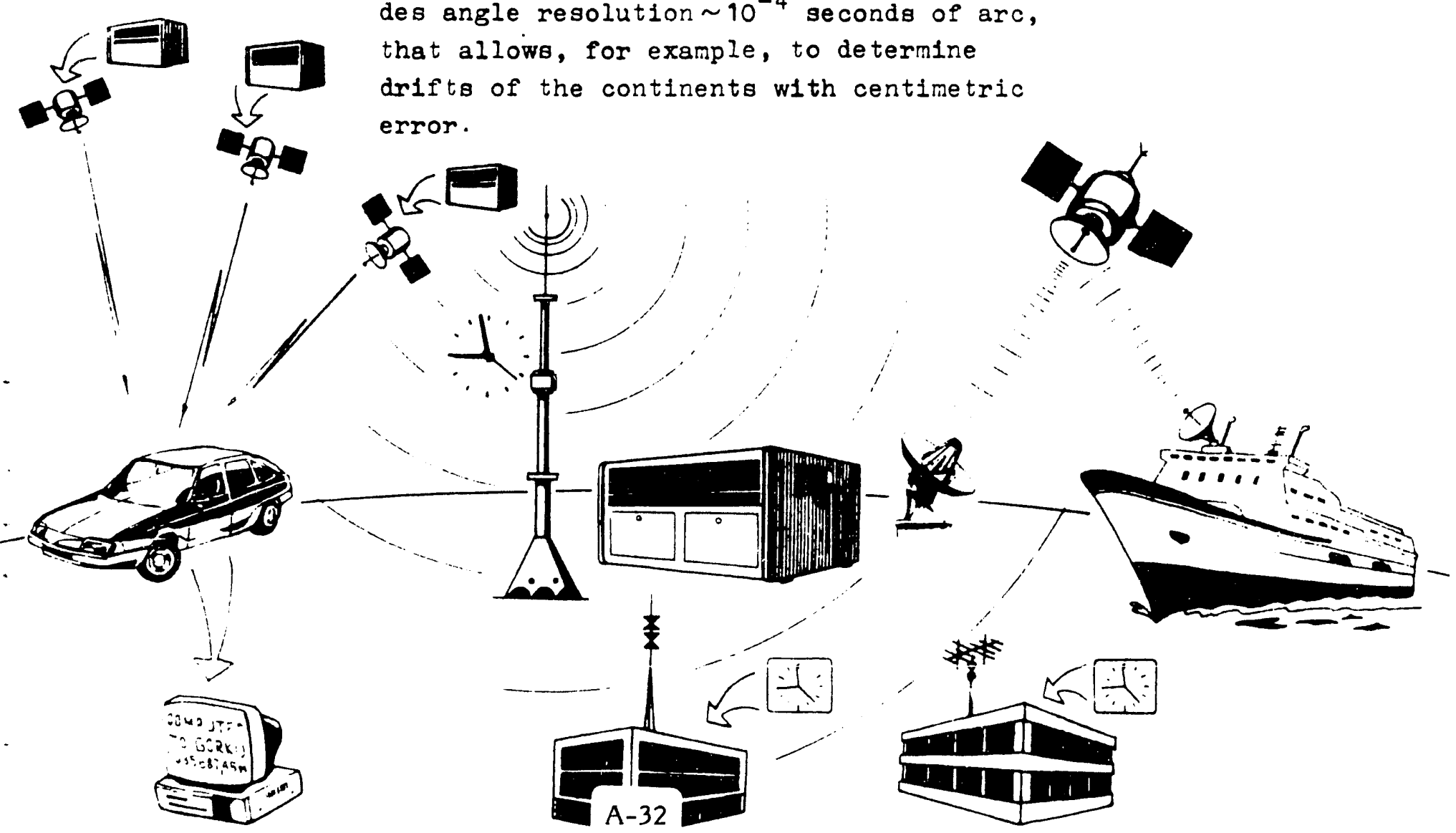


Output frequency: $1 \mathrm{~Hz}, 5 \mathrm{Mriz}$

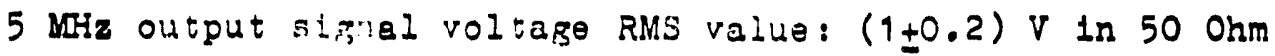

Frequency accurasy: not more than $\pm 1.5 \cdot 10^{-12}$

$1 \mathrm{~Hz}$ pulse signal parame ters: amplitude - not less than $2.5 \mathrm{~V}$ in 50 ohm

$$
\begin{aligned}
& \text { pulse width - 10-20 } \mu \mathrm{s} \\
& \text { rise lime - not wore than } 30 \mathrm{~ns}
\end{aligned}
$$

$5 \mathrm{MHz}$ outpu: algnal frequency otabll1ty (at $\pm 1^{\circ} \mathrm{C}$ amblent temperature change in operaing iemperature range $5-40^{\circ} \mathrm{C}$ ) not more than: $3 \cdot 10^{-12}(1 \mathrm{~s})$

$$
\begin{aligned}
& 7 \cdot 10^{-13}(108) \\
& 2 \cdot 10^{-13}(1008) \\
& 7 \cdot 10^{-14}(10008) \\
& 5 \cdot 10^{-14}(1 \mathrm{~h}) \\
& 3 \cdot 10^{-14}(24)
\end{aligned}
$$

Additional frequency variation at $1^{\circ} \mathrm{C}$ amblent temperature change wlthln operating temperature range: not more than $\pm 2 \cdot 10^{-14}$

Frequency reproduclbility: not more than $3 \cdot 10^{-13}$

Compensator Erequency retuning range: not les8 than $1 \cdot 10^{-10}$ at in atep $1 \cdot 10^{-14}$

$1 \mathrm{Ha}$ pulso train 10 synchronized by positivo polarity pulses with repetition rato 18, pulse width not wore than $2 \mu s$, amplitude not $108 \theta$ than $2.5 \nabla$ in 50 Obm; synchronization accuracy - not more than \pm 200 no.

5 MHz output signal harmonic component ouppression: not lese than $26 \mathrm{~dB}$

SSB phase nolse power spectral density 18 not more then: $-125 \mathrm{~dB} / \mathrm{kz}$ (20Rz)

$-195 \mathrm{~dB} / \mathrm{Bz}$ (330 $\mathrm{BS})$

$-140 \mathrm{~dB} / \mathrm{kg}$ (1kHis)

Power: $(220 \pm 22) \mathrm{V},(50 \pm 0.5) \mathrm{Hz}$

$$
(220 \pm 11) \mathrm{V},(115 \pm 5.75) \mathrm{V},\left(400_{-12}^{+28}\right) \mathrm{Hz}
$$

In case of emergency the hydrogen standard automatically owltches to DC exterasi power supply 22-30 V, preserving specifications

Power consump:10n: AC - not more than $140 \mathrm{VA}$

$D C$-not more than $90 \mathrm{~W}$

We1ght: $55 \mathrm{~kg}$

Dimens1028: $280 \times 480 \times 555 \mathrm{~mm}$

Operating conditions: environmental temperature: $5-40^{\circ} \mathrm{C}$

relative humldity: up to $80 \%$ at $25^{\circ} \mathrm{C}$

atmospheric preseure: 84-106 kPa

$(630-795 \mathrm{~mm} \mathrm{Hg})$

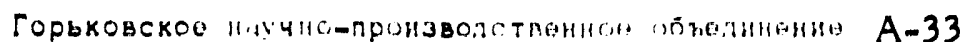

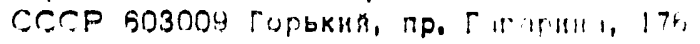

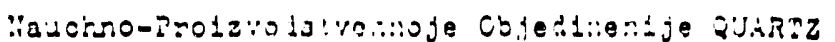

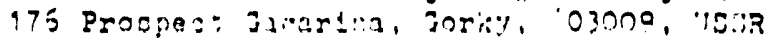


(blank)

A -34 


\section{APPENDIX B}

\section{ABOUT THE AUTHORS}

Robert F. C. Vessot (Panel Chairman). Dr. Vessot is Senior Physicist at the Harvard-Smithsonian Center for Astrophysics. He received his BA (1951), MSc (1954), and PhD (1956) in physics from McCill University in Montreal, Canada. He was a Telecommunications Officer in the Royal Canadian Air Force from 1951 to 1954. Upon completion of his PhD, he joined the Department of Sponsored Research at the Massachusetts Institute of Technology where he worked on atomic clocks under the late professor J. R. Zacharias. From 1960 to 1969, he was Manager of Maser Research and Development at Varian Associates/Hewlett Packard. Dr. Vessot is presently Senior Physicist at the Harvard-Smithsonian Center for Astrophysics. Since 1969, he has been the Principal Investigator of the Smithsonian Astrophysical Observatory (SAO) Hydrogen Maser Laboratory Group, which has built all of the hydrogen masers for NASA's Deep-Space Network and has supported radio astronomical very long baseline interferometry (VLBI) activities worldwide. He has worked on the development of atomic hydrogen masers since 1961 and has contributed to the success of the hydrogen maser as the most stable oscillator now available. In 1978, Dr. Vessot received the NASA Medal for Exceptional Scientific Achievement for his work as Principal Investigator on the gravitational redshift experiment (NASA's Gravity Probe-A). This test confirmed Einstein's predictions of the effects of relativistic gravitation on the rate of clocks at a precision of $70 \mathrm{ppm}$. Dr. Vessot has been the author and co-author of more than 100 articles and has made many contributions of books. He is a member of the American Physics Society and Sigma Xi.

David W. Allan. Mr. Allan is Senior Scientist in the Time and Frequency Division at the National Institute of Standards and Technology (NIST) in Boulder, Colorado. He is also the leader of the group which generates the NIST atomic time scales and coordinates international time and frequency comparisons. He received a BS in physics from Brigham Young University in 1960 and an MS in physics from the University of Colorado in 1965. Mr. Allan joined the Atomic Frequency and Time Standards Section of the National Bureau of Standards in 1960, where he worked with ammonia beam masers and related quantum electronic devices. Since 1962, his work has been directed toward the development of the atomic time scale systems for the National Institute of Standards and Technology (formerly NBS). He has received numerous awards for his work and has travelled extensively. In May 1985, he was invited to the International Astronomical Union Symposium on Relativity and Celestial Mechanics in Leningrad to present a paper on "Coordinate Time in the Vicinity of the Earth." Mr. Allan is a member of the Scientific Research Society of America, Sigma Xi, the International Radio Consultative Committee (CCIR), the International Radio Scientific Union (URSI), and the International Astronomical Union.

Stuart J. B. Crampton. Dr. Crampton is the Barclay Jermain Professor of Natural Philosophy and the Director of the Bronfman Science Center at Williams College. He received a BA (1958) with highest honors from Williams College, a BA, Hon. (1960) from Oxford University, an MA (1961), and a PhD (1964) from Harvard University, and an MA (1965) from Oxford University. He began his teaching career as an Assistant Professor at Williams College in 1965. He has served as a visiting Associate Professor at the Massachusetts Institute of Technology and has held positions as Chercheur Associé and Professeur Associé at École Normale Superieure in Paris. Dr. Crampton has received numerous awards and honors, most recently the 1989 American i'hysical Society Award for Research at an Undergraduate Institution. He has authored or co-auth red over 30 research articles. He is a Fellow of the American Physical Society, a member of Phi i eta Kappa, Sigma Xi, and the American Association of Physics Teachers. 
Leonard S. Cutler. Dr. Cutler has worked as Distinguished Contributor at Hewlett-Packard Laboratories in Palo Alto, California since 1957. He was previously the Director of the Superconductivity Laboratory in the Corporate Research Laboratories. He received BS, MS, and PhD degrees in physics from Stanford University in 1958, 1960, and 1966, respectively. He has been active in the areas of precision ratio transformers, frequency meters, quartz, and atomic frequency standards including passive cesium, rubidium and mercury ion standards, and hydrogen masers. He has also been involved with frequency stability theory, special and general relativity, laser interferometry for precision distance measurement, magnetic bubble memories, disc memories, superconductivity and photonics. He was responsible for the design of the first all solid-state cesium beam frequency standard and has been deeply involved since with theory and design of cesium beam tubes and frequency standard electronics. In 1974, Dr. Cutler was elected Fellow of the Institute of Electrical and Electronics Engineers (IEEE) for his work in atomic frequency standards and frequency stability. He is a member of the Magnetics Society of the IEEE and is also a member of the American Association for the Advancement of Science, Sigma Xi, and the American Physical Society. In 1984, he received both the Morris E. Leeds Award and the Centennial Award from the IEEE. In 1987, he was elected to the National Academy of Engineering.

Robert H. Kern. Mr. Kern is the founder and President of Kernco, Inc., a company established in 1978, which specializes in advancing the atomic clock state of the art through development and production of high-performance, high-reliability satellite borne clocks. Prior to forming Kernco, Mr. Kern was the President of Frequency and Time Systems, Inc., a company he founded in 1971. In the 1970s, Mr. Kern developed and patented a new generation of ruggedized cesium bearn tube resonators suitable for use in space and severe dynamic environment situations. Before 1971, he was with the Frequency and Time Divi...un of Hewlett-Packard Company, which, in 1967, acquired the Quantum Electronics Division of Varian Associates, Mr. Kern's former employer. Prior to his association with Varian and Hewlett-Packard, he held the positions of Senior Scientific Executive for EG\&G, Inc., and Senior Research and Development Engineer for CBS Electronics. Mr. Kern holds BSEE and MSEE degrees from the School of Electrical Engineering, Cornell University.

Arthur O. McCoubrey. Dr. McCoubrey is the Acting Chief of the Temperature and Pressure Division at the National Institute of Standards and Technology (NIST) Center for Chemical Technology. He received a BS degree in applied physics from the California Institute of Technology in 1943, and a PhD in physics from the University of Pittsburgh in 1953. Dr. McCoubrey has been engaged in the management of NIST programs for reference standards, methods of measurement for physical quantities, calibrations, and related research and measurement services for industry and government. In this connection, he was Director of the Institute for Basic Standards from 1974 to 1978 . He then served as Associate Director for Measurement Services in the newly organized National Measurement Laboratory. From 1984 to 1988, he was responsible for industrial outreach activities in the Center for Basic Standards. Prior to his government service, he was employed for more than 30 years in the electrical and electronics industries. His early industrial work at Westinghouse Research Laboratories included microwave measurement methods, research in atomic and molecular physics, and research on superconducting phenomena at millimeter-wave frequencies. Later, for a period of 15 years, mainly at Varian Associates, Dr. McCoubrey was engaged in the development of frequency and time standards based on cesium atomic beam resonators, rubidium buffer gas cell resonators, and atomic hydrogen masers. He also served as Director of Central Research at Varian Associates from 1968 to 1972 . He is a member of the American Physical Society, the Instrument Society of America, and the American Society for Quality Control, and is a Fellow of the Institute of Electrical and Electronics Engineers. 
Joseph D. White. Dr. White currently heads the Advanced Technology Section of the Space Applications Branch at the Naval Research Laboratory (NRL). He received a BS in physics in 1969 from Western Kentucky University, and an MS and $\mathrm{PhD}$ in physics from American University in 1974 and 1981, respectively. From 1969 to 1973, he served as an officer in the US Army Signal Corps at the Army Night Vision Laboratory. While with the Army, he performed research in bolometric infrared detectors and imaging systems. Following completion of active duty, Dr. White joined the NRL in 1973 as a research physicist. From 1977 to 1983, he continued with cesium clock development in support of the Global Positioning System satellites, the initial space hydrogen maser work, and the construction of the NRL Clock Test Facility. In 1982, he assumed responsibility for the development of hydrogen masers for space and ground applications in GPS. In 1987, he became head of the NRL clock development section, with responsibilities in development, testing, and applications of clocks and timekeeping. Dr. White serves as a technical consultant to major programs, including the US Naval Observatory, MILSTAR, and the MARK XV IFF System. He is a member of the technical program committees for the Precise Time and Time Interval Meeting and the Annual Symposium of Frequency Control, the two major conferences for the time and frequency community. 
(blank)

B-4 


\section{APPENDIX C GLOSSARY OF ABBREVIATIONS, ACRONYMS, AND SPECIALIZED TERMS}

\begin{tabular}{|c|c|}
\hline A & ampere \\
\hline $\mathrm{AC}$ & alternating current \\
\hline accuracy & $\begin{array}{l}\text { the degree of conformity of a measured or calculated value to its } \\
\text { definition (see uncertainty) }\end{array}$ \\
\hline aging & $\begin{array}{l}\text { the systematic change in frequency with time caused by internal } \\
\text { changes in the oscillator }\end{array}$ \\
\hline AN SSSR & Akademiya nauk (Academy of Sciences of the USSR) \\
\hline ANSI & American National Standards Institute \\
\hline API & American Petroleum Institute \\
\hline AS USSR & Academy of Sciences of the USSR \\
\hline ASME & American Society of Mechanical Engineers \\
\hline ASTM & American Society for Testing and Materials \\
\hline $\mathrm{AT}$ & a type of crystal cut \\
\hline BIH & Bureau International de L'Heure \\
\hline BIPM & Bureau International de Poid de Mesure \\
\hline BT & a type of crystal cut \\
\hline CCDS & $\begin{array}{l}\text { Comitè Consultatif de la Seconde, or Consultative Committee for } \\
\text { the Definition of the Second }\end{array}$ \\
\hline CCIR & International Radio Consultative Committee \\
\hline CCITT & $\begin{array}{l}\text { Consultative Committee in International Telegraphy and Tele- } \\
\text { phony }\end{array}$ \\
\hline CGSC & Civil GPS Service Committee \\
\hline $\mathrm{CH}_{4}$ & methane \\
\hline om & centimeter \\
\hline $\mathrm{CO}_{2}$ & carbon dioxide \\
\hline COCOM & $\begin{array}{l}\text { Coordinating Committee: a multilateral body for establish- } \\
\text { ing/implementing controls for technology transfer to the Soviet } \\
\text { Union and Eastern Europe based upon national security considera- } \\
\text { tions; includes all of the NATO countries (except Iceland) and } \\
\text { Japan. }\end{array}$ \\
\hline Cs & cesium \\
\hline $\mathrm{dB}$ & decibel \\
\hline DC & direct current \\
\hline drift & the systematic change in frequency of an oscillator with time \\
\hline EIA & Electronic Industries Association \\
\hline
\end{tabular}




\begin{tabular}{|c|c|}
\hline error & the difference of a value from its assumed correct value \\
\hline FC & a type of crystal cut \\
\hline FEP & fluorinated ethylene-propylene \\
\hline FM & frequency modulation \\
\hline frequency instability & $\begin{array}{l}\text { the spontaneous and/or environmentally caused frequency change } \\
\text { within a given time interval }\end{array}$ \\
\hline fs & femtosecond \\
\hline $\mathrm{GHz}$ & gigahertz \\
\hline GLONASS & Soviet Global Orbiting Navigation Satellite System \\
\hline GOES & $\begin{array}{l}\text { weather satellites used by the (US) National Oceanographic and } \\
\text { Atmospheric Administration }\end{array}$ \\
\hline Gosstandart & State Committee for Standards (USSR) \\
\hline GPS & US Global Positioning System \\
\hline $\mathrm{H}$-maser & hydrogen maser \\
\hline $\mathrm{He}$ & helium \\
\hline $\mathrm{He}-\mathrm{Ne}$ & helium-neon \\
\hline $\mathrm{HF}$ & high frequency \\
\hline $\mathrm{Hg}$ & mercury \\
\hline $\mathrm{Hz}$ & hertz \\
\hline $\mathrm{I}_{2}$ & iodine \\
\hline IAU & International Astronomical Union \\
\hline IEEE & Institute of Electrical and Electronics Engineers \\
\hline IEN & Instituto Elettrotechnico Nazionale (Italy) \\
\hline ION & Institute of Navigation \\
\hline IR & infrared \\
\hline IRE & Institute of Radio Engineers \\
\hline IT & a type of crystal cut \\
\hline JPL & Jet Propulsion Laboratory \\
\hline $\mathrm{K}$ & Kelvin \\
\hline $\mathrm{kHz}$ & kilohertz \\
\hline $\mathrm{km}$ & kilometer \\
\hline LF & low frequency \\
\hline LT & a type of crystal cut \\
\hline
\end{tabular}




\begin{tabular}{|c|c|}
\hline maser & microwave amplification by stimulated emission of radiation \\
\hline MCXO & microcomputer-controlled crystal oscillator \\
\hline MF & medium frequency \\
\hline $\mathrm{MHz}$ & megahertz \\
\hline MIT & Massachusetts Institute of Technology \\
\hline $\mathrm{mK}$ & milliKelvin \\
\hline $\mathrm{mph}$ & miles per hour \\
\hline NASA & National Aeronautics and Space Administration \\
\hline NBS & National Bureau of Standards (now NIST) \\
\hline $\mathrm{NCO}$ & numerically controlled oscillator \\
\hline NIST & National Institute of Standards and Technology \\
\hline $\mathrm{rm}$ & nanometer \\
\hline NOAA & $\begin{array}{l}\text { National Oceanographic and Atmospheric Administration (United } \\
\text { States) }\end{array}$ \\
\hline NPL & National Physical Laboratory (United Kingdom) \\
\hline NPO & Scientific Production Association \\
\hline NRL & Naval Research Laboratory \\
\hline ns & nanosecond \\
\hline ONR & Office of Naval Research \\
\hline $\mathrm{O}_{\mathrm{s}} \mathrm{O}_{4}$ & osmium tetraoxide \\
\hline $\mathrm{pA}$ & picoamp \\
\hline PM & phase modulation \\
\hline ppm & part per million \\
\hline precision & $\begin{array}{l}\text { the degree of mutual agreement among a series of individual } \\
\text { measurements (often, but not necessarily, expressed by the standard } \\
\text { deviation) }\end{array}$ \\
\hline ps & picosecond \\
\hline PTB & $\begin{array}{l}\text { Physiakalish-Tecknische Bundesanstalt, or the (West) German } \\
\text { NBS }\end{array}$ \\
\hline PTFE & polytetrafluoroethlyene \\
\hline QND & quantum nondemolition \\
\hline Qz & quartz \\
\hline R\&D & research and development \\
\hline $\mathrm{Rb}$ & rubidium \\
\hline reproducibility & $\begin{array}{l}\text { (a) with respect to a set of in dependent devices of the same design, } \\
\text { the ability of these devices to produce the same value; (b) with }\end{array}$ \\
\hline
\end{tabular}


RF

$\mathrm{RMC}$

RMS

RT

SA

SAO

$\mathrm{SC}$

$\mathrm{SF}_{6}$

SI

SPTFS

SS

STFS

TAI

TCXO

$\mathrm{THz}$

UkSSR

uncertainty

URSI

USNO

UTC

VAC

VDC

VLBI

VNIIFTRI

VNIIM

VNIIMS

WWV respect to a single device, put into operation repeatedly without adjustments, the ability to produce the same value

radio frequency

radio-meteor channel

root-mean-square

a type of crystal cut

selective availability

Smithsonian Astrophysical Observatory

a type of crystal cut

sulphur hexafluoride

Système International d'Unitès, or System International

State Primary Time and Frequency Standard

secondary standard

State Time and Frequency Service

International Atomic Time

temperature-compensated crystal oscillators

terahertz

Ukrainian Soviet Socialist Republic (Ukraine)

the limits of the confidence interval of a measured or calculated quantity

International Radio Scientific Union

United States Naval Observatory

Coordinated Universal Time

volts $\mathrm{AC}$

volts $\mathrm{DC}$

very long baseline interferometry

Physical Technical and Radio Engineering Measurements AllUnion Scientific Institute, Mendeleyevo (Moscow)

Metrology All-Union Scientific Research Institute im. D. I. Mendeleyev, AS USSR, Leningrad

Metrological Services All-Union Scientific Research Institute, Moscow

The call letters of a radio station maintained by NIST to provide standard radio and audio frequencies and other technical services (precision time signals, radio propagation disturbance warnings); the station broadcasts on 2.5, 5, 10, 15, 20, 25, 30, and $35 \mathrm{MHz}$ at various locations. 
WWVB

WWVH

$\mu \mathrm{m}$

$\mu s$
NIST radio station at Boulder, Colorado, broadcasting services similar to WWV at $60 \mathrm{kHz}$.

NIST radio station at 'iaui, Hawaii, broadcasting services similar to those of WWV on 5,10 , and $15 \mathrm{MHz}$.

micrometer

microsecond 
(blank)

C-6 


\section{APPENDIX D SOVIET JOURNALS CITED IN TEXT/REFERENCES}

For readers not familiar with the Soviet technical literature, a key to the abbreviated titles of the Soviet serial literature cited in this report is provided below. The titles of the Englishlanguage translations used are listed in bold print and the original Russian-language titles are in italics. When a given Soviet technical journal is published in more than one commercial translation, the English title for the same Soviet source may vary with the publisher. If translations have been made privately (for example, government agency translations), the titles may also vary. Frequently, English titles are not literal translations of the original Russian. Therefore, knowledge of the Russian title of a journal may be necessary to identify reference materials.

\section{Abbreviation}

Cosmic Res.

Dokl. AS USSR

Instrum. Exp. Tech.

J. Appl. Spectrosc.

JETP Lett.

Meas. Tech.

Opt. Spectrosc.

Radio Eng. Electron. Phys.

Radiophys. Quantum Electron

Sov. Astron. Lett.

Sov. J. Commun. Technol. Electron.
English Translation Title/Original Russian Title

Cosmic Research

Kosmicheskiye issledovaniya

Doklady Academy of Sciences

Doklady Akademii nauk SSSR

Instruments and Experimental Techniques

Pribory $i$ tekhnika eksperimenta

Journal of Applied Spectroscopy

Zhurnal prikladnoy spektroskopii

JETP Letters

Pis'ma Zhurnal eksperimental'noy $i$ teoreticheskoy fiziki

Measurement Techniques

Izmeritel'naya tekhnika

Optics and Spectroscopy

Optiki i spektroskopiya

Radio Engineering and Electronics Physics (superseded by the Soviet Journal of Communication Technology and Electronics) Radiotekhnika $i$ elektronika

Radiophysics and Quantum Electronics Izvestiya vysshikh uchebnykh zavedeniy (VUZ), Radiofizika

Soviet Astronomy-Letters

Pis'ma $v$ Astronomicheskiy zhurnal

Soviet Journal of Communications Technology and Electronics (formerly/until 1985 Radio Engineering and Electronics Physics)

Radiotekhinika i elektronika 
Sov. J. Quantun Electron.

Sov. Phys.-JETP

Sov. Phys.-Solid State

Sov. Phys.-Tech. Phys.

Sov. Phys.-Dokl.

Sov. Phys.-Usp.

Sov. Tech. Phys. Lett.

Telecomm. Radio Eng.

Theor. Math. Phys.

Vestn. Leningr. Univ. Math.
Soviet Journal of Quantum Electronics

Kvantovaya elektronika

Soviet Physics-JETP (Journal of Experimental \& Theoretical Physics)

Zhurnal eksperimental'noy $i$ teoreticheskoy fiziki

Soviet Physics-Solid State

Fizika tverdogo tela

Soviet Physics-Technical Physics

Zhurnal tekhnicheskoy fiziki

Soviet Physics-Doklady

Doklady AN SSSR

Soviet Physics-Uspekhi

Uspekhi fizicheskikh nauk

Soviet Technical Physics Letters

Pis'ma $v$ Zhurnal tekhnicheskoy fiziki

Telecommunications and Radio Engineering Elektrosvyaz and Radiotekhnika-selected translations from both journals

Theoretical and Mathematical Physics

Teoreticheskaya $i$ matematicheskaya fizika

Vestnik of the Leningrad University: Mathematics Vestnik Leningradskogo universiteta, Matematika, mekhanika, astronomiya 


\section{APPENDIX E \\ SOVIET RESEARCH FACILITIES CITED IN TEXT}

(* full information not available)

Atomic Energy Institute im. I. V. Kurchatov, Moscow

(Institut atomnoy energii imeni I. V. Kurchatova/IAE)

Engineering Physics Institute, Moscow

(Moskovskiy inzhenerno-fizicheskiy institut/MIFI)

Gor'kiy R\&D Instrument Making Institute, Gor'kiy

(Gor'kovskiy nauchno-issledovatel'skiy priborostroitel'nyy institut)

High Temperatures Institute, AS USSR, Moscow

(Institut vysokikh temperatur, AN SSSR/IVTAN)

Leningrad Polytechnic Institute im. M. I. Kalinin, Leningrad

(Leningradskiy politekhnicheskiy institut imeni M. I. Kalinina/LPI)

Leningrad Radiotechnical Scientific Research Institute, Leningrad*

Main Astronomical Observatory, AS UkSSR, Kiev

(Glavnaya astronomicheskaya observatoriya, AN UKSSR/GAO)

Metrological Services All-Union Scientific Research Institute, Moscow

(Vsesoyuznyy nauchno-issledovatel'skiy institut metrologicheskikh sluzhb/VNIIMS)

Metrology All-Union Scientific Research Institute im. D. I. Mendeleyev, AS USSR, Leningrad (Vsesoyuznyy nauchno-issledovatel'skiy institut metrologii imeni D. I. Mendeleyeva, AN SSSR/VNIIM)

Moscow State University im. M. V. Lomonosov, Moscow

(Moskovskiy gosudarstvennyy universitet imeni M. V. Lomonosova)

Physical-Technical and Radio Engineering Measurements All-Union Scientific Research Institute, Mendeleyevo (Moscow)

(Vsesoyuznyy nauchno-issledovatel'skiy institut fiziko-tekhnicheskikh $i$ radiotekhnicheskikh izmereniy/VNIIFTRI)

Physical-Technical Institute im. A. F. Ioffe, AS USSR, Leningrad

(Fiziko-tekhnicheskiy institut imeni A. F. Ioffe, AN SSSR/FTIAN)

Physics Institute im. P. N. Lebedev, AS USSR, Moscow

(Fizicheskiy institut imeni P. N. Lebedeva, AN SSSR/FIAN)

Radio Scientific Research Institute, Leningrad

(Leningradskiy otdel' nauchno-isslevotatel'skiy institut radio/LONIIR)

Radiophysics and Electronics Institute, AS ArSSR, Yerevan

(Institut radiofiziki $i$ elektroniki, AN ArSSR/IRFE) 
Scientific Production Association (NPO) "Quartz," Gor'kiy

(Nauchno-proizvodstvennoye ob"yedeniye 'Kvartz')

Semiconductor Physics Institute, AS USSR, Novosibirsk

(Institut fiziki poluprovodnikov, SO AN SSSR/IFP)

Space Research Institute, AS USSR, Moscow

(Institut kosmicheskikh issledovaniy, AN SSSR/IKI)

Spectroscopy Institute, AS USSR, Troitsk (Krasnaya Pakhra)

(Institut spektroskopii, AN SSSR)

Theoretical Physics Institute im. L. D. Landau, AS USSR, Chernogolovka (Moscow)

(Institut teoreticheskoy fiziki imeni L. D. Landau, AN SSSR/ITF)

Thermal Physics Institute, Siberian Branch AS USSR, Novosibirsk

(Institut teplofiziki, SO AN SSSR) 


\section{APPENDIX F \\ FASAC REPORT TITLES}

( * asterisk before title indicates report is classified)

(completed)

FY-82/83

* Soviet High-Pressure Physics Research

Soviet High-Strength Structural Materials Research

Soviet Applied Discrete Mathematics Research

* Soviet Fast-Reaction Chemistry Research

FY-84

Soviet Physical Oceanography Research

Soviet Computer Science Research

Soviet Applied Mathematics Research: Mathematical Theory of Systems, Control, and Statistical Signal Processing

Selected Soviet Microelectronics Research Topics

* Soviet Macroelectronics (Pulsed Power) Research

FY-85 FASAC Integration Report: Selected Aspects of Soviet Applied Science

Soviet Research on Robotics and Related Research on Artificial Intelligence

Soviet Applied Mathematics Research: Electromagnetic Scattering

* Soviet Low-Energy (Tunable) Lasers Research

Soviet Heterogeneous Catalysis Research

Soviet Science and Technology Education

Soviet Space Science Research

FASAC Special Report: Effects of Soviet Education Reform on the Military

Soviet Tribology Research

Japanese Applied Mathematics Research: Electromagnetic Scattering

Soviet Spacecraft Engineering Research

Soviet Exoatmospheric Neutral Particle Beam Research

Soviet Combustion Research

Soviet Remote Sensing Research and Technology

Soviet Dynamic Fracture Mechanics Research

FY-86/89 Soviet Magnetic Confinement Fusion Research

Recent Soviet Microelectronics Research on III-V Compound Semiconductors

Soviet Ionospheric Modification Research

Soviet High-Power Radio Frequency Research

Free-World Microelectronic Manufacturing Equipment 


\section{(completed/con't.)}

FY-86/89

FASAC Integration Report II: Soviet Science as Viewed by Western Scientists Chinese Microelectronics

Japanese Structural Ceramics Research and Development

System Software for Soviet Computers

Soviet Image Pattern Recognition Research

West European Magnetic Confinement Fusion Research

Japanese Magnetic Confinement Fusion Research

* Soviet Research in Low-Observable Materials

FASAC Special Study: Comparative Assessment of World Research Efforts on Magnetic Confinement Fusion

FASAC Special Study: Defense Dependence on Foreign High Technology

Soviet and East European Research Related to Molecular Electronics

Soviet Atmospheric Acoustics Research

Soviet Phase-Conjugation Research

FASAC Special Study: Soviet Low Observable/Counter Low Observable Efforts: People and Places

Soviet Oceanographic Synthetic Aperture Radar Research

Soviet Optical Processing Research

FASAC Integration Report III: The Soviet Applied Information Sciences in a Time of Change

Soviet Satellite Communications Science and Technology

Soviet Precision Timekeeping Research and Technology

$$
\text { (in production) }
$$

FY-86/89 West European Nuclear Power Generation Research and Development

* Radiation Cone Research

FASAC Special Study: Non-US Artificial Neural Network Research

FY-90/91 Soviet Chemical Propellant Research and Development

Soviet Optoelectronics Research

Soviet Parallel Processing Research

Soviet Nonlinear Dynamics Research

* Foreign Research Relevant to Countering Stealth Vehicles

Soviet Research in Penetration Mechanics

Soviet and Free World Bandpass Radome Research and Development

FASAC Special Study: Non-US Artificial Neural Network Research

Soviet Macroelectronics (Pulsed Power) Research 
(blank)

F-3 
(blank)

F-4 



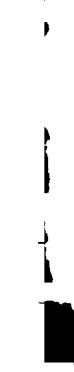


